\title{
Globalization of labor services
}

Citation for published version (APA):

Vallizadeh, E. (2016). Globalization of labor services. [Doctoral Thesis, Maastricht University]. Datawyse / Universitaire Pers Maastricht. https://doi.org/10.26481/dis.20160118ev

Document status and date:

Published: 01/01/2016

DOI:

$10.26481 /$ dis.20160118ev

Document Version:

Publisher's PDF, also known as Version of record

\section{Please check the document version of this publication:}

- A submitted manuscript is the version of the article upon submission and before peer-review. There can be important differences between the submitted version and the official published version of record.

People interested in the research are advised to contact the author for the final version of the publication, or visit the DOI to the publisher's website.

- The final author version and the galley proof are versions of the publication after peer review.

- The final published version features the final layout of the paper including the volume, issue and page numbers.

Link to publication

\footnotetext{
General rights rights.

- You may freely distribute the URL identifying the publication in the public portal. please follow below link for the End User Agreement:

www.umlib.nl/taverne-license

Take down policy

If you believe that this document breaches copyright please contact us at:

repository@maastrichtuniversity.nl

providing details and we will investigate your claim.
}

Copyright and moral rights for the publications made accessible in the public portal are retained by the authors and/or other copyright owners and it is a condition of accessing publications that users recognise and abide by the legal requirements associated with these

- Users may download and print one copy of any publication from the public portal for the purpose of private study or research.

- You may not further distribute the material or use it for any profit-making activity or commercial gain

If the publication is distributed under the terms of Article $25 \mathrm{fa}$ of the Dutch Copyright Act, indicated by the "Taverne" license above, 
Globalization

\section{of Labor Services}

Ehsan Vallizadeh

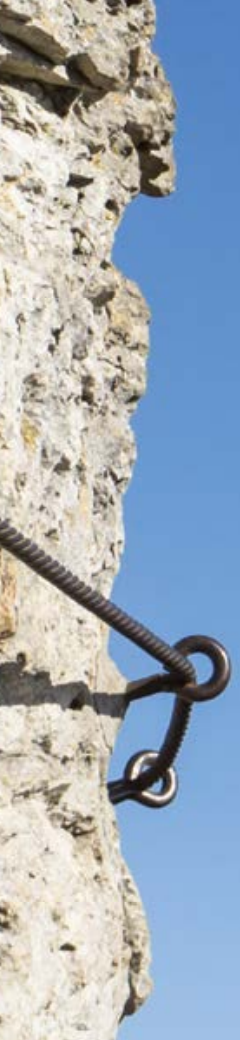


Copyright (C)Ehsan Vallizadeh, Maastricht 2016

All rights reserved. No part of this publication may be reproduced, stored in a retrieval system, or transmitted, in any form, or by any means, electronic, or mechanical, including photocopying, recording or otherwise, without prior permission in writing from the author.

This book was typeset by the author using $\mathrm{H}_{\mathrm{H}} \mathrm{T}_{\mathrm{E}} \mathrm{X}$.

Cover by Ehsan Shirangi and Burim Suli.

Published by Datawyse - Universitaire Pers Maastricht

ISBN 978-94-6159-527-0 


\title{
Globalization of Labor Services
}

\author{
DISSERTATION
}

to obtain the degree of Doctor at Maastricht University, on the authority of the Rector Magnificus,

Prof. dr. L.L.G. Soete

in accordance with the decision of the Board of Deans,

to be defended in public

on Monday January 18, 2016, at 14:00 hours

\section{Ehsan Vallizadeh}

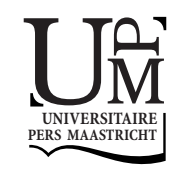




\section{Supervisor:}

Prof. dr. Joan Muysken

\section{Co-supervisor:}

Dr. Thomas Ziesemer

\section{Assessment Committee:}

Prof. dr. Bart Verspagen (chair)

Dr. Neil Foster-McGregor (UNU-MERIT)

Prof. dr. Maarten Goos (Utrecht University School of Economics)

Prof. dr. Hans-Jörg Schmerer (University of Hagen)

This research was funded by the Maastricht Research School of Economics of Technology and Organizations (METEOR). 
To my parents, Mahwash and Masoud my wife, Sercan

my sons, Kian and Navid

for their neverending love and support 



\section{Acknowledgments}

As a novice climber, I learned quickly that carefulness, determination, endurance, and skills are almost all key attitudes that one should bring to climb a rock face. What is left in this "almost" may be described as the necessity for anyone to successfully complete a challenging climbing tour. Whenever finding the next good grip or proceeding with the next safe step becomes difficult, it is the support by and the trust in your belay partner that allows you to move forward. He can provide directions and advice on how to balance the power between the fingers, the arms, and the feet to overcome the tricky points, as, from a distance, he can see all the difficulties on the way up. He gives a feeling of safety and ease to help you reach the top. I learned also fairly quickly that regardless of how many marks exist on the climbing wall and how many people have already climbed a certain route, you must find your own path on the way to the top.

Reflecting upon the years spent working on my dissertation, I came to see an analogy between the world of climbing and the realm of doing research. Generating a research idea, formulating and fine-tuning the research question, conducting the analyses, transforming these into written results, and putting it all together into a full research paper roughly capture the process of conducting academic research. The progress along the different stages and the overall academic achievement are determined by a combination of individual effort - characterized by the key attitudes mentioned above - and the environmental conditions that set the direction of this path.

I consider myself as quite fortunate to have had the opportunity to do research and to be surrounded by inspiring people these last few years. All these people had a considerable influence on both my personal and professional development, and in some way helped to accomplish this dissertation. First of all, I want to express my gratitude to my supervisors, Joan Muysken and Thomas Ziesemer. Joan, I would like to thank you for your patience and allowing me the freedom I needed to develop myself and to explore my own research interests. Your advice, encouragement and guidance helped me a lot to structure my thoughts, to overcome every difficulty I encountered, and, in fact, to write this thesis. Thomas, your door was always open to me, allowing me to discuss any issues related to students and teaching, or to my research projects, or to simply brainstorm about new research ideas. Thank you for your invaluable advice and guidance in addressing all the detailed technical points in my thesis. Using the climbing analogy above: I was lucky to have had two belay partners, helping me find my way throughout the years of my PhD. 
Next, I would like to thank the members of the assessment committee: Bart Verspagen, Neil Foster-McGregor, Maarten Goos, and Hans-Jörg Schmerer for their careful reading and constructive comments on my dissertation. Further, I would like to express my gratitude to Herbert Brücker for his insightful discussions and helpful suggestions on earlier versions of my second chapter. Herbert, you gave me the opportunity to join your research team at IAB in 2013. Working with you on various policy-related projects on the topic of migration has helped me very much to better understand the relevance and importance of research in the public discourse. Thank you for this insight.

Special thanks go out to Fleur, Silvana, and Sylvia: thank you for your invaluable help and assistance regarding administration and organization, as well as for all the free candy and coffee/tea provided at the secretariat. Fleur, thank you very much for taking care of all phone calls and sending out all the letters to MBB, board of deans, committee, etc. in the last stage of my dissertation, and that everything worked out so smoothly.

My PhD years in Maastricht would have been less enjoyable without my friends and colleagues. Special thanks go to Nils Holinski for his support and for helping me find an apartment and settle in Maastricht in my first year. Moreover, I am thankful to Aline, Anne, CaiCai, Elnaz, Hossein, Iman, Lenard, Lennart, Mona, Nikita, Norbert, Ron, Sajid, Thomas, and Ulf for many interesting research-related and less-scientific relaxing discussions during coffee breaks and lunches, drawing and music sessions in the office, and many more pleasant moments outside university. Bart and Tyas, thank you for being my paranymphs and for all your support throughout the years. I am also deeply indebted to my friend Jiwoong Lee for the advice, suggestions and insights he shared with me.

Special thanks go to my dear friend Burim Suli for introducing me to the world of climbing, all the private climbing sessions in Frankonian Switzerland, and making the motive for the cover of my thesis possible. I also wish to thank my dear long-standing friend Ehsan Shirangi for his kind help in designing the cover. Talking about climbing, I would like also to thank Nyree and Joost for wonderful hiking memories in the Alpes, all the "pre-knocking" moments, and all the good talks, helping me clear my mind.

Much gratitude I owe to my family. I would like to thank my parents, Mahwash and Masoud, my brother Hanif, and my sister Sara for their unconditional love and continuous support for everything. I cannot thank you enough for encouraging me during this experience. I also want to thank my parents-in-law, Emine and Ali, my brother-in-law, Erdem, my sister-in-law Gülcan and her husband Güven for your continuous support.

Last but certainly not least, I am most grateful to my wife Sercan. You are the true proof that behind a successful man there is indeed a strong woman. Without you I would not have been able to achieve this stage in my life. I know it was not always easy for you when I spent nights and weekends behind the laptop. Sercan, ich möchte mich bei dir insbesondere für deine bedingungslose Liebe und Unterstützung, für deine positive und fröhliche Lebensart und dafür, dass du immer an mich geglaubt hast, von ganzem Herzen bedanken. Kian und Navid, ihr seid mein Leben und mein ganzer Stolz. Danke, dass ihr immer dafür gesorgt habt, dass ich all den Arbeitsstress so schnell vergessen konnte. I am very lucky to have you all in my life; you made me who I am today.

Ehsan Vallizadeh, Nuremberg, December 2015 


\section{Contents}

1 Introduction 1

1.1 Motivation ........................... 1

1.2 Perceptions about globalization of the labor market . . . . . . . . . . 3

1.3 Facts and hypotheses . . . . . . . . . . . . . . 6

1.4 Outline of the thesis . . . . . . . . . . . . . . . . 11

2 Migration, Unemployment, and Skill Downgrading 15

2.1 Introduction . . . . . . . . . . . . . . . . . 15

2.2 Literature review . . . . . . . . . . . . . . . . . . . 17

2.2.1 Employment and wage effects: the canonical approach . . . . . 17

2.2.2 Absorption channels of immigrants in the economy . . . . . . . . 18

2.2.3 Advances in technology and the polarization of the labor market . 19

2.2.4 Transferability of human capital and the assimilation of immigrants 19

2.2.5 Brain drain, gain, and waste: The perspective of the sending countries 20

2.3 Stylized facts . . . . . . . . . . . . . . . . . . . . . . 21

2.4 Theoretical framework . . . . . . . . . . . . . . . . . . . . . . . . . . . . . . . . 23

2.4.1 Firm optimization problem . . . . . . . . . . . . 25

2.4.2 Wage setting and labor market frictions . . . . . . . . . . 27

2.5 The general equilibrium solution . . . . . . . . . . . . . . . . . . . . . . . 29 
2.5.1 Changes in the steady state . . . . . . . . . . . . . . 30

2.5.2 Comparative statics with exogenous benefits . . . . . . . . . . . 31

2.5.3 Comparative statics with endogenous benefits . . . . . . . . . . 34

2.6 Numerical assessment . . . . . . . . . . . . . . . . . . . . . . . . . . . 37

2.6.1 Migration scenarios . . . . . . . . . . . . . . . . . . . . . . . . . . . . . . . . . . . . . 38

2.6.2 Simulation results . . . . . . . . . . . . . . . . . . . 39

2.7 Conclusion . . . . . . . . . . . . . . . . . . . . . 42

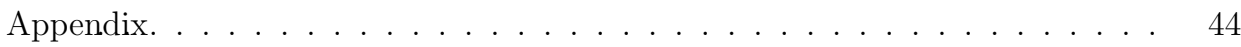

2.A Empirical analysis . . . . . . . . . . . . . . . . . . . . 44

2.A.1 Data . . . . . . . . . . . . . . . . 44

2.A.2 Estimation of the correlation between over-qualification and unemployment rates . . . . . . . . . . . . . . . . . 44

2.A.3 Estimation of the role of institutions on the relationship between unemployment and downgrading . . . . . . . . . . . . 45

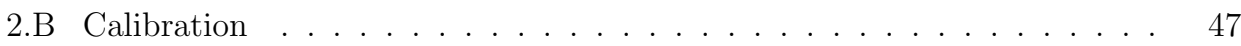

2.B.1 Cobb-Douglas production technology . . . . . . . . . . . 47

2.B.2 Benchmark statistics and calibration . . . . . . . . . . . 48

2.C Bargaining solution and unique equilibrium . . . . . . . . . . . . 50

2.C.1 Bargaining solution . . . . . . . . . . . . . . 50

2.C.2 Unique equilibrium condition under endogenous benefits . . . . . 50

3 Offshoring of Medium-skill Jobs, Polarization, and Productivity Effect 55

3.1 Introduction . . . . . . . . . . . . . . . . 55

3.2 Task assignment . . . . . . . . . . . . . . . . . . . . . . 59

3.2.1 Allocation of domestic tasks . . . . . . . . . . . . . 59

3.2.2 Allocation of offshored tasks . . . . . . . . . . . . . 61

3.3 Production technology and labor demand . . . . . . . . . . . . . . . 64

3.3.1 Optimal labor demand . . . . . . . . . . . . . . . . 64

3.3.2 Equilibrium marginal cost . . . . . . . . . . . . . 66

3.4 General equilibrium solution . . . . . . . . . . . . . . 67

3.5 Comparative statics . . . . . . . . . . . . . . . . . 68

3.5.1 Increasing offshorability and task reallocation . . . . . . . . . 69

3.5.2 Offshoring and distributional effects . . . . . . . . . . . . . . . . 70

3.5.3 Offshoring-induced cost-efficiency effect . . . . . . . . . . . . . 74

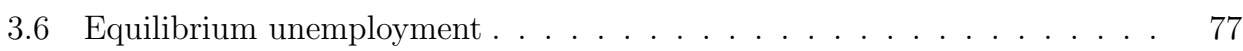

3.6 .1 Minimum wage regime . . . . . . . . . . . . . . . . . . . . 77

3.6.2 Endogenous labor supply . . . . . . . . . . . . . . . 80

3.7 Conclusion . . . . . . . . . . . . . . . . . . . 82

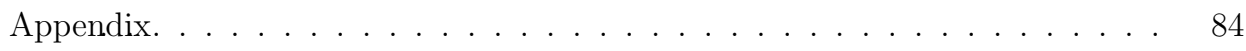

3.A Derivation of equilibrium conditions . . . . . . . . . . . . . . . . 84

3.A.1 Firm optimization problem . . . . . . . . . . . . . . 84

3.A.2 Cost-efficiency effect . . . . . . . . . . . . . . . 84 


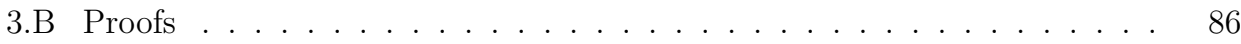

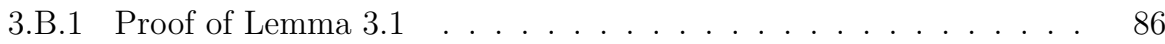

3.B.2 Proof of Lemma $3.2 \ldots \ldots \ldots \ldots$. . . . . . . . . . . 86

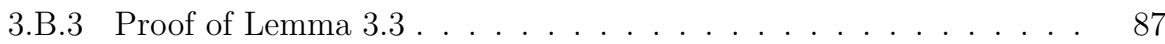

3.B.4 Proof of Proposition 3.1: Uniqueness of task margins . . . . . . . 88

3.C Extension: Alternative offshoring scenarios of domestic job tasks . . . . 92

3.C.1 Offshoring low-skill-intensive domestic job tasks . . . . . . . . . . 92

3.C.2 General equilibrium solution . . . . . . . . . . . . . . . . 94

3.C.3 Comparative statics: Easier offshoring and skill-task reallocation . 95

3.C.4 Offshoring low-skilled job tasks and productivity effect . . . . . . 96

3.C.5 Offshoring high-skill-intensive domestic job tasks . . . . . . . . . 98

3.C.6 Offshoring and domestic income distribution effect . . . . . . . . . 99

3.D Supplementary general equilibrium solutions . . . . . . . . . . . . . . . 101

3.D.1 Comparative statics for changes in offshoring margins . . . . . . 101

3.D.2 Comparative statics for changes in minimum wage . . . . . . . . 102

3.D.3 Comparative statics for changes in offshoring under minimum wage scheme . . . . . . . . . . . . . . . . 103

3.D.4 Comparative statics for changes in offshoring under endogenous low-skilled labor supply . . . . . . . . . . . . . . . . . . . . . . 104

4 Immigration, Technology Adoption and Wage Inequality 107

4.1 Introduction . . . . . . . . . . . . . . . . . . 107

4.2 Literature review . . . . . . . . . . . . . . . . . . . . . . . . . . 110

4.3 Theoretical framework . . . . . . . . . . . . . . . . . . 111

4.3.1 Aggregate consumption good, final goods, and technology adoption 112

4.3.2 Intermediate goods market . . . . . . . . . . . . . . . . . 114

4.4 Characteristics of equilibrium . . . . . . . . . . . . . . 115

4.5 Immigration and changes in technology adoption margins . . . . . . . . . 119

4.6 Immigration and distributional effect . . . . . . . . . . . . . . . . . . . . 121

4.6.1 Low-skill and high-skill immigration and distributional effect . . . 123

4.6.2 Medium-skill immigration and distributional effect . . . . . . . . . 125

4.7 Conclusion . . . . . . . . . . . . . . . . . . . . 126

Appendix. . . . . . . . . . . . . . . . . . . . . . . . 128

4.A Proofs . . . . . . . . . . . . . . . . . . . . . . 128

4.A.1 Proof of Lemma 4.1 . . . . . . . . . . . . . . . . . . 128

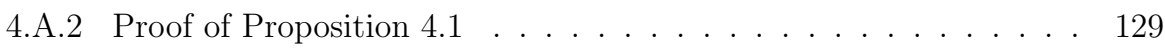

4.A.3 Proof of Proposition $4.2 \ldots \ldots \ldots$. . . . . . . . . . . . . 130

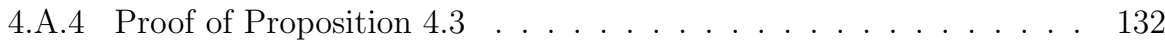

4.B Derivation of equilibrium solutions . . . . . . . . . . . . . . . . . 132

4.B.1 Demand for intermediate goods . . . . . . . . . . . . . . . . 132

4.B.2 Derivation of the mass of monopolists . . . . . . . . . . . . . 133

4.B.3 Derivation of aggregate sectoral output . . . . . . . . . . . . . 133

4.B.4 Derivation of relative wages . . . . . . . . . . . . . . . . . 134 
5 Immigration, Offshoring, Tasks and Technology Adoption 137

5.1 Introduction . . . . . . . . . . . . . . . . . . . . . . . 137

5.2 Related literature . . . . . . . . . . . . . . . . . . . . . 140

5.3 The Model . . . . . . . . . . . . . . . . . . . . . . . . . . . . . . . 142

5.3 .1 Basic environment . . . . . . . . . . . . . . . . . . . 142

5.3.2 Task allocation between immigrants and natives . . . . . . . . . . 144

5.4 Characteristics of equilibrium . . . . . . . . . . . . . . . 145

5.5 Comparative statics . . . . . . . . . . . . . . . . . . . . . . . . . . 149

5.5.1 Immigration and technology adoption . . . . . . . . . . . . . . 149

5.5.2 Immigration and native wage structure . . . . . . . . . . . . . . . 151

5.6 Offshoring of intermediate inputs . . . . . . . . . . . . . . . . . . . . . . . . . . . . 157

5.6.1 Offshoring and technology adoption . . . . . . . . . . . . . 160

5.6 .2 Offshoring and native wage structure . . . . . . . . . . . . . 161

5.7 Conclusion . . . . . . . . . . . . . . . . . . . . 164

Appendix. . . . . . . . . . . . . . . . . 166

5.A Proofs . . . . . . . . . . . . . . . . . 166

5.A.1 Proof of Lemma 5.1 . . . . . . . . . . . . . . 166

5.A.2 Derivation of equilibrium labor composite and marginal costs . . . 167

5.A.3 Proof of Lemma 5.2 . . . . . . . . . . . . . . . . . . . . . . 169

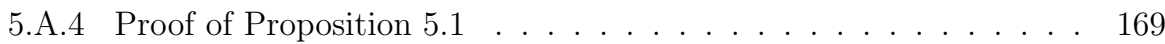

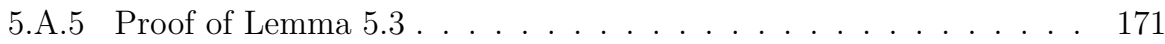

5.A.6 Proof of Proposition $5.4 \ldots \ldots \ldots \ldots$. . . . . . . . . . . 171

5.A.7 Proof of Proposition $5.6 \ldots \ldots \ldots$

5.B Derivation of equilibrium solutions . . . . . . . . . . . . . . . 173

5.B.1 Derivation of equilibrium mass of monopolists $A_{k} \ldots \ldots 173$

5.B.2 Derivation of aggregate sectoral output . . . . . . . . . . . . . . 174

5.B.3 Derivation of relative wages . . . . . . . . . . . . . 175

5.B.4 Derivation of immigration-induced changes in relative range of final goods .......................... 177

5.B.5 Impact of immigration on native wage structure . . . . . . . . . . 179

5.B.6 Derivation of $\hat{A}_{M} \ldots \ldots \ldots$. . . . . . . . . . . . . 181

$\begin{array}{ll}\text { Bibliography } & 183\end{array}$

$\begin{array}{ll}\text { Samenvatting (Summary in Dutch) } & 191\end{array}$

$\begin{array}{ll}\text { Curriculum Vitae } & 195\end{array}$ 


\section{List of Figures}

1.1 Share of developing countries in advanced countries' trade . . . . . . . . 2

1.2 Global labor supply . . . . . . . . . . . . . . . . . . . . 3

1.3 Net-flow of migration to major European receiving countries . . . . . . 4

1.4 Perception regarding immigration and adverse wage effects . . . . . . . 5

1.5 Perception regarding trade and wage effects . . . . . . . . . . . 5

1.6 Share of foreign workers in total labor force of receiving country . . . . 7

1.7 Pattern of offshoring inputs in advanced countries by skill intensity . . 8

1.8 Relative Employment of Nonproduction/Production Workers in the U.S. Manufacturing ...................... 8

1.9 Changes in employment shares by occupation 1993-2006 in 16 European countries and the USA . . . . . . . . . . . . . . . . . 9 9

2.1 Net replacement rate and regression coefficients of low-skilled unemployment versus medium-skilled over-qualification rates . . . . . . . . . . 22

2.2 Properties of the manufacturing-wage curve . . . . . . . . . . . . . 51

2.3 Properties of the services-wage curve . . . . . . . . . . . . . . 52

2.4 Unique equilibrium . . . . . . . . . . . . . . . . . . 53

3.1 Equilibrium task allocation . . . . . . . . . . . . . . . 63

3.2 Offshoring medium-skilled job tasks and wage polarization . . . . . . 71

3.3 Offshoring low-skilled job tasks and skill-biased wage effect . . . . . . . 72

3.4 Offshoring high-skilled job-tasks and unskill-biased wage effects . . . . . 73

3.5 Unique equilibrium of low- and high-skilled task margins . . . . . . . . 89

3.6 Offshorability of low-skill-intensive job-tasks and skill-task allocation . . 93

3.7 Offshoring high-skilled job tasks and skill-task allocation . . . . . . . . 99

4.1 Changes in real male wages in the US by percentile . . . . . . . . . . . 108

4.2 Structure of model . . . . . . . . . . . . . . . . . . . . . . 112

4.3 Final goods price and technology productivity schedules . . . . . . . . . 114

4.4 Medium-skill immigration and changes in the equilibrium technology margins . . . . . . . . . . . . . . . . . . . 120 
4.5 Equilibrium technology allocation . . . . . . . . . . . . . . . 129

5.1 Immigrants and task specialization . . . . . . . . . . . . . . . . . 139

5.2 Interaction between productivity and labor supply effects . . . . . . . 156 


\section{List of Tables}

2.1 Educational attainments of immigrants in selected countries of destination 21

2.2 Evolution of employment in Europe by educational attainment and oc-

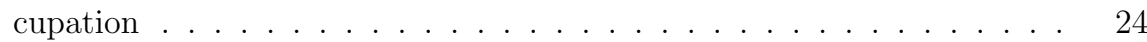

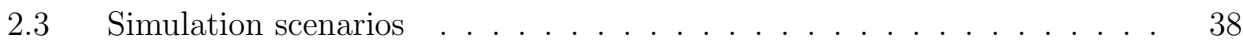

2.4 Simulation of labor market effects of migration . . . . . . . . . . . . . 41

2.5 Correlation between medium-skilled over-qualification and low-skilled unemployment rates . . . . . . . . . . . . . . . . . . . 45

2.6 Correlation between net replacement rate and the estimated unemploymentdowngrading relationship . . . . . . . . . . . . . . . . . 46

2.7 Calibrated and benchmark equilibrium values for the industries . . . . . 48

2.8 Labor market benchmark values . . . . . . . . . . . . . . . . . . . . . . 49

2.9 Equilibrium Conditions . . . . . . . . . . . . . . . . . . 54

5.1 Immigration and native wage structure, if $\sigma<\epsilon \ldots \ldots . \ldots 157$ 



\section{Introduction}

\subsection{Motivation}

Over the past two decades, the pace and trends of globalization have changed considerably, characterizing an increasing pattern of integration of national goods and labor markets into the global market. This new pattern of globalization is explained by two major events. On the one hand, advances in information and communication technology, combined with the gradual removal of barriers to trade and capital flows, have made it possible for production and work processes to lose the locational viability for a growing number of goods and services. This has enabled firms in advanced countries to unbundle the production process into a global value added chain by exploiting the comparative advantage differences across countries. The accession of emerging and developing countries to the global markets has considerably intensified the trade relations with advanced economies (cf. Spence, 2011). For example, since the early 1990s, the share of developing countries' products in total manufacturing import of advanced economies has increased by 50 percent, driven largely by China (see Figure 1.1).

On the other hand, political changes as well as institutional and economic reforms have induced a substantial transformation of countries like Brazil, Russia, India, China (BRIC) and the former Eastern European bloc countries, into economies with high accumulation rates of human capital and advanced technology, and improving business infrastructure. This has enabled their large economies and labor forces to participate in open market economies. As depicted in Figure 1.2, the integration of these emerging countries has led to a marked growth in the effective global labor supply, with most of the increase taking place after 1990. About half this increase has been attributed to the rise in the working-age population in East Asia, while South Asia and Central and Eastern Europe contributed moderately. ${ }^{1}$

\footnotetext{
${ }^{1}$ For detailed discussions regarding trends in and implications of globalization of labor see IMF (2007).
} 
Figure 1.1: Share of developing countries in advanced countries' trade
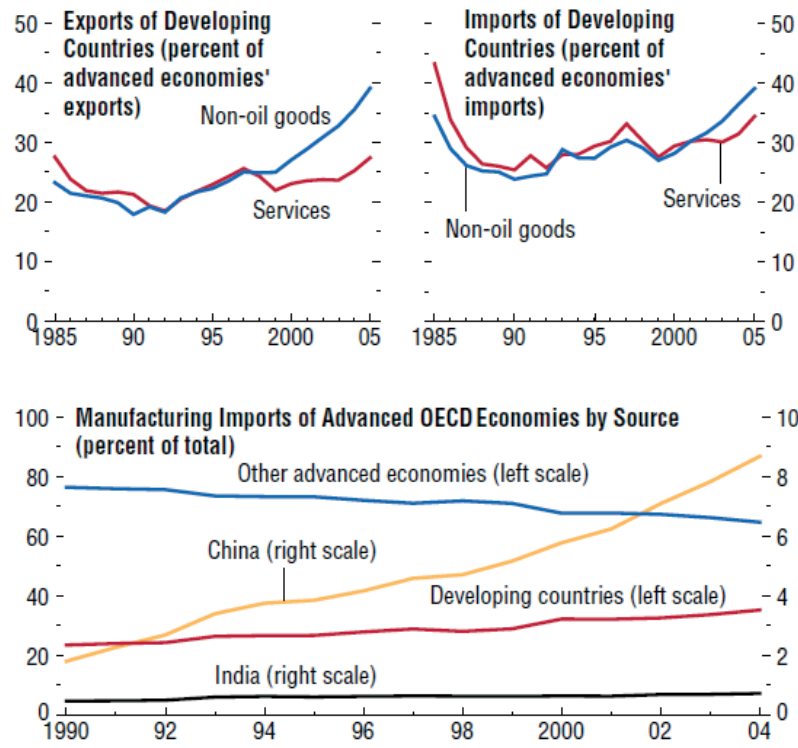

Source: (IMF, 2007, Figure 5.3).

These developments have also led to a sizable increase in migration flows to advanced countries, accompanied with substantial changes in the skill structure of immigrants. According to OECD (2007a), today more than 115 million immigrants live in OECD countries, constituting about $10 \%$ of the population. Moreover, the number of highlyeducated immigrants has grown by $70 \%$ over the past decade to exceed 31 million in the OECD area (OECD, 2007a). Particularly, in the course of the two major rounds of the EU-Enlargement towards Central, Eastern and Southeastern European countries ${ }^{2}$ the former EU countries have experienced an influx of immigration from these new member states of the EU. Since the early 2000s, the average annual net-flow of migration in some large European economies (Germany, Italy, Spain, and the UK) has ranged between 130,000 and 400,000 persons.

Figure 1.3 depicts these trends in net-migration flows for some of the main European destination countries. In 2012, the total net-flow of migration to some major European destination countries ${ }^{3}$ reached 1.1 million persons. Thus, migration has become a constant factor in the economic and social landscape in many advanced countries. Therefore, the aim of this thesis is to address the labor market implications of these global developments for the workforce in the advanced countries.

\footnotetext{
${ }^{2}$ The first round of EU-Enlargement refers to the accession of eight Central and Eastern European countries in May 2004: Estonia, Czech Republic, Hungry, Latvia, Lithuania, Poland, Slovak Republic, and Slovenia. The second round of EU-Enlargement refers to the accession of Bulgaria and Romania to the EU in January 2011.

${ }^{3}$ These include Austria, Belgium, Germany, Italy, the Netherlands, Spain, Sweden, and the UK.
} 
Figure 1.2: Global labor supply
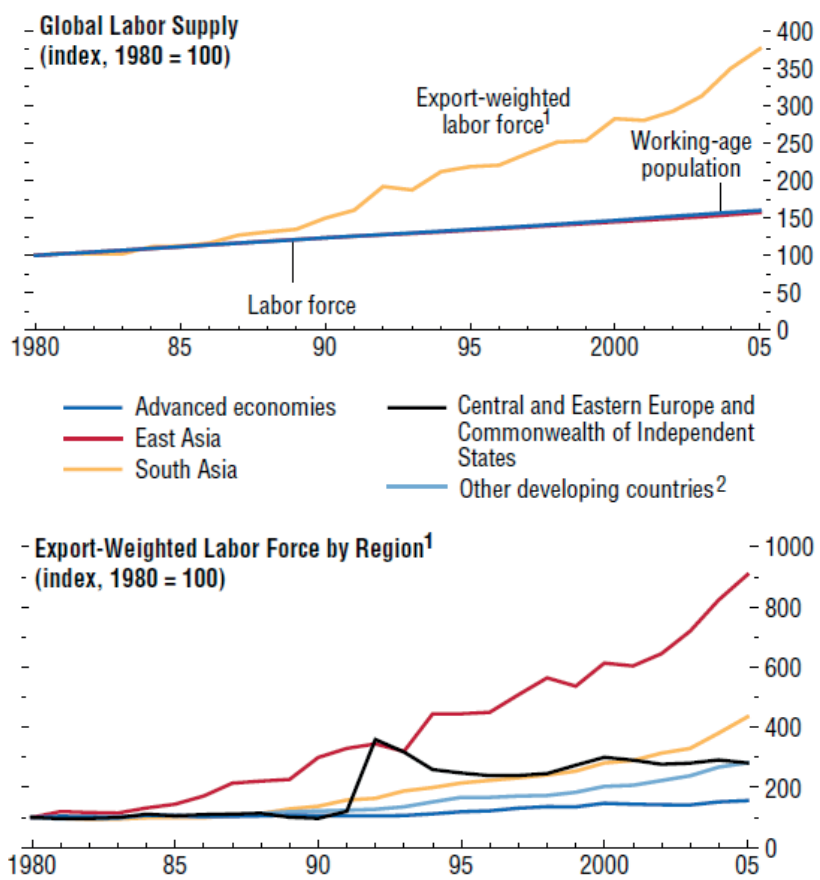

Source: (IMF, 2007, Figure 5.1).

1 National labor forces scaled by export-to-GDP ratios.

2 Western Hemisphere, Middle East and North Africa, and sub-Saharan Africa.

\subsection{Perceptions about globalization of the labor market}

At the turn of the 21th century, this soaring global integration of goods and labor markets has enabled advanced countries to access the large pool of labor supply both through imports of workers (i.e. immigration) and of goods and services (i.e. offshoring). This development has received wide attention by policymakers and the media, portraying it as a threat to millions of workers and jobs in many advanced countries. For clarification, I proceed with a description of these concerns and perceptions in the advanced countries.

Plotting data from the European Social Survey, Figure 1.4 shows the perception regarding immigration-induced adverse wage effects. The results suggest that there are, indeed, some concerns regarding the detrimental wage consequences of immigration among residents in many European receiving countries. For example, 48 per cent of residents in France, and about 37 per cent in Austria, Italy, Germany, and the U.K, respectively, agree with the statement that immigration lowers wages in the receiving countries. Moreover, controlling for educational background, mainly individuals with low or no qualification perceive that immigration lowers the wages, and thus are more likely to be in favor of limiting immigration flows (cf. Scheve and Slaughter, 2001). 
Figure 1.3: Net-flow of migration to major European receiving countries
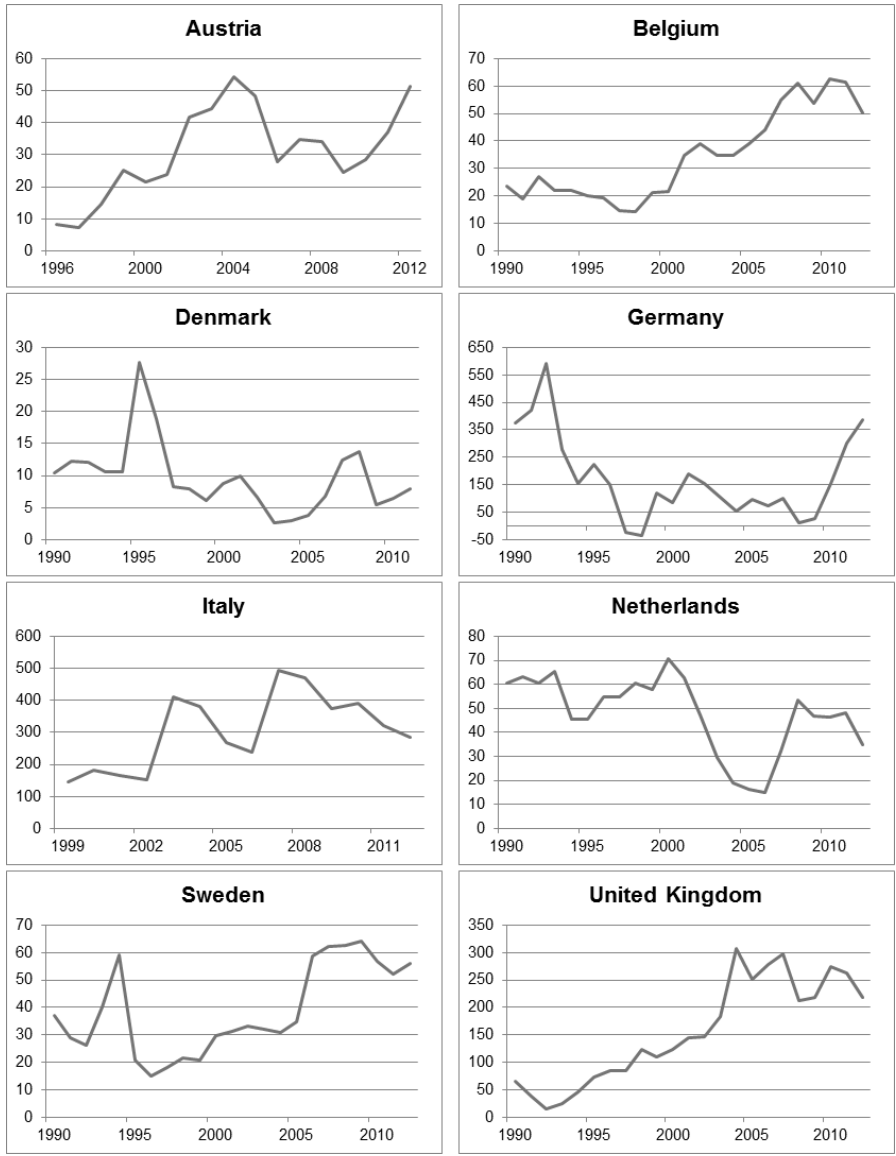

Source: OECD.Stat, International Migration Database, own calculation.

The debate regarding offshoring received an extraordinary amount of attention in the US, when the Economic Report of the President was released in early 2004. The peak of the hysteria was when Gregory Mankiw, serving at that time as chairman of President Bush's Council of Economic Advisers, said in a statement: "I think outsourcing is a growing phenomenon, but it's something that we should realize is probably a plus for the economy in the long run. [...] [O]utsourcing is just a new way of doing international trade" (Mankiw and Swagel, 2006, pp. 1031-1032). This position has been met with a storm of harsh criticism, and outsourcing became synonymous with job loss in America. ${ }^{4}$

Most prominently, Blinder (2006) has suspected that offshoring of jobs from rich to developing countries is "business as usual", or "another routine expansion of international trade", contrary to Mankiw's claim. He estimates that offshoring service jobs could potentially cause about 30 to 40 million American jobs to disappear to emerging countries

\footnotetext{
${ }^{4}$ The report "The great hollowing-out myth" in The Economist, Feb 14th, 2004, exemplifies the political issue received by outsourcing in America.
} 
Figure 1.4: Perception regarding immigration and adverse wage effects

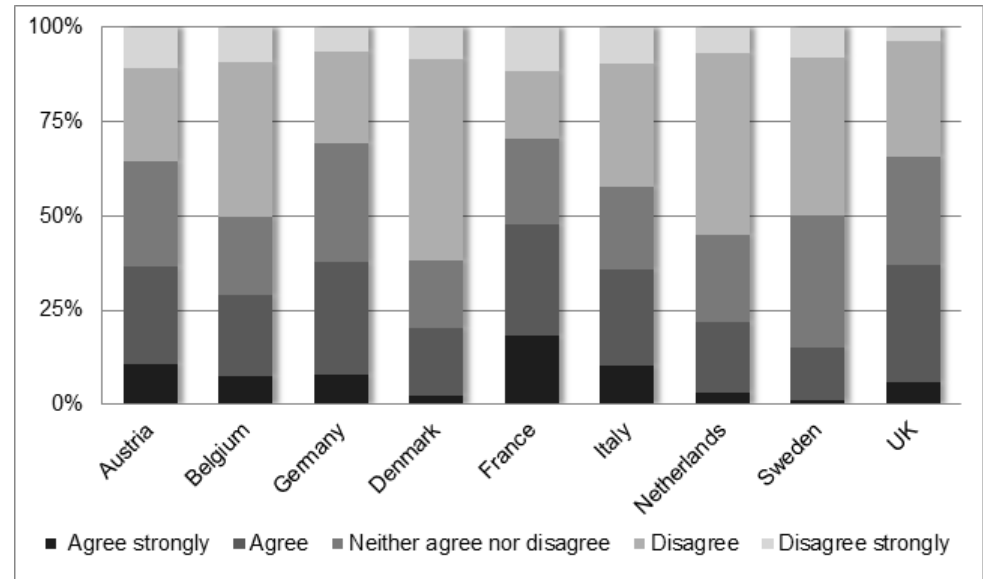

Notes: The figure shows the distribution of answers to the question whether average wages are generally brought down by immigration for a selected group of EU countries.

Source: European Social Survey, first wave, 2002, own calculation.

like India (Blinder, 2009b), and thus induce a large "quantitative change [that] brings about a qualitative change" for workers in the U.S. and other advanced economies (Blinder, 2006, p. 113). A similar prediction has been made by the McKinsey Global Institute, extrapolating from a study of eight industrial sectors ${ }^{5}$ that 1.5 million service jobs were offshored from developed to low-wage countries in 2003, and that this number will rise to 4.1 million by 2008, as reported in The Economist (June 30, 2005).

Figure 1.5: Perception regarding trade and wage effects

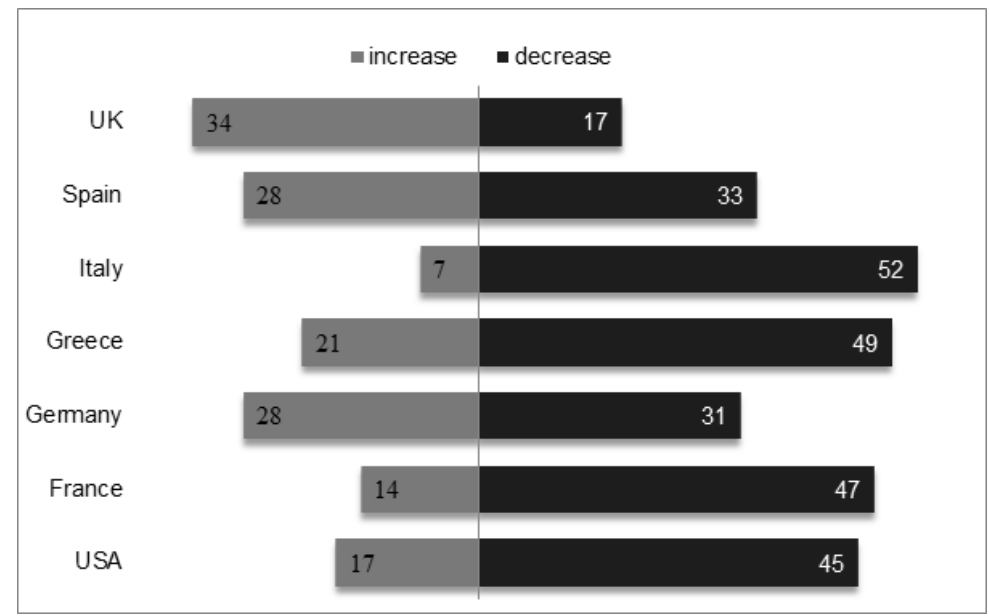

Notes: The figure shows the distribution of answers to the question whether trade with other countries leads to an increase or decrease in wages (results for "no difference" not shown). Source: PEW Research Center, Spring2014 Global Attitudes survey, Q28, own calculation.

\footnotetext{
${ }^{5}$ These include engineers, finance and accounting professionals, analysts, life science researchers, doctors, nurses, and generalists.
} 
Such dramatic forecasts caused widespread alarm in many developed countries. In a survey by PEW Research Center in 2006, 77 percent of respondents perceived offshoring of jobs as the major problem hurting American workers compared to other trends, like immigration, automation, modern communication technology and declining unionization. More generally, the results of the global attitudes survey highlight the common concern of people in many advanced countries that free trade will harm the labor market conditions (Figure 1.5).

Therefore, the most frequently asked questions concerning the public, policy makers, and researchers, are whether the globalization of labor is adversely affecting wages and employment in the advanced economies, and through which channels the domestic economy absorbs and adjusts to the global labor supply shock. Providing clear answers to these questions is of importance from both an academic and a policy perspective. It improves our understanding regarding potential adjustment mechanisms in the goods and labor markets. In addition, it allows providing clear guidance for implementing effective policy measures to reap the benefits, while minimizing the costs of immigration and offshoring, and thus helping to bring more objectivity to the emotional and heated public debates. In that respect the analysis in this thesis provides a systematic decomposition of various potential adjustment mechanisms through which the labor market and the economy are affected by immigration and offshoring.

\subsection{Facts and hypotheses}

In order to address the public concerns, it is useful to get first an idea about how wage and employment structures have evolved in advanced countries over the past two decades. Do they contrast the past pattern? What are the characteristics of these trends? And, related to this question, to what extent are they linked to recent features of globalization of labor? Indeed, as shown below, the descriptive evidence depicts a contrasting picture of the recent trends in wages and employment in many advanced countries.

Looking first at immigration, immigrants constitute a substantial proportion of the labor force in many advanced countries today. As shown in Figure 1.6, the share of foreign workers in total labor force of major European destination countries has significantly increased over the past two decades. Moreover, the number of immigrants with tertiary education attainment has dramatically increased over past two decades, an increase by 70 percent between 2000 and 2010 in OECD countries (Bonfanti and Xenogiani, 2014). Despite the substantial improvements in the educational attainment of immigrants, their attachment to the receiving country's labor market exhibits high entry barriers upon arrival. These labor market frictions are usually associated with lower labor market participation and particularly with a high incidence of skill downgrading, referring to a mismatch between the acquired educational attainment and the required skill level for an occupation, of immigrants relative to natives. A growing number of empirical studies has provided evidence regarding the key factors contributing to these detrimental labor market outcomes such as lack of language proficiency and host country specific skills (e.g. knowledge about social norms, institutions, etc.), migration status (temporary versus permanent), imperfect transferability of human capital across countries, as well as 
Figure 1.6: Share of foreign workers in total labor force of receiving country

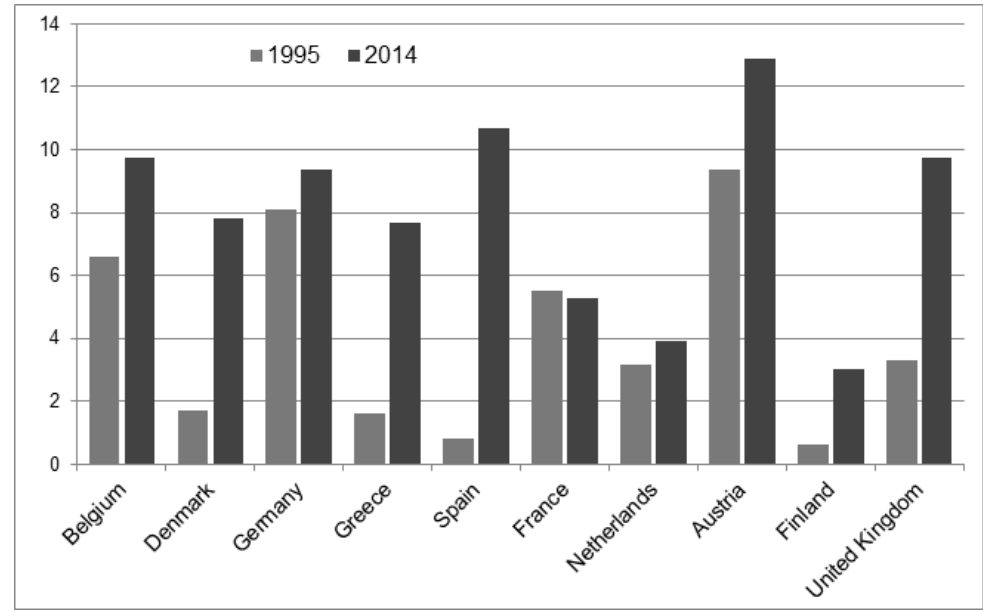

Notes: 1999-2014 for the Netherlands. Source: Eurostat, own calculation.

labor market discrimination practices (Bonfanti and Xenogiani, 2014).

More precisely, immigrants with tertiary education have on average an over-qualification rate that is 12 percentage points higher than that of native-born with the same characteristics in Europe, and 1.5 percentage points higher in the United States (Damas de Matos and Liebig, 2014). Thus, collecting these facts, i.e. increasing number of skilled immigrants in conjunction with skill downgrading, one would prematurely argue that

Hypothesis 1. Immigration-induced increase in skill-downgrading incidence leads to a displacement effect of low-skilled workers from the labor market.

Understanding the determinants and the interaction between immigration-induced changes in the skill structure of the receiving country and the labor market outcomes of low-skilled workers, and particularly how labor market institutions shape the direction of immigration shock is the core objective of chapter 2. As is argued below, under a flexible labor market regime medium-skilled immigration generates a complementarity effect between skill-downgrading and low-skill unemployment.

Turning to the offshoring phenomenon, in many advanced countries, a striking observation has been witnessed since the early 1990s. The rise in offshoring has been characterized mostly by imports of skilled rather than unskilled inputs (Figure 1.7). This captures the widespread consensus that the recent pattern in the globalization process is reflecting a new stage of international trade, deflecting the international labor market competition towards jobs and tasks performed mostly by better educated and skilled workforce in the advanced countries.

A growing number of studies has sought to provide evidence regarding the labor market impact of offshoring. Figure 1.8 shows that the relative employment of skilled (i.e. nonproduction) workers compared to unskilled (i.e. production) workers in the U.S. manufacturing sector has fallen since the early 1990s, a finding that "is strongly suggestive 
Figure 1.7: Pattern of offshoring inputs in advanced countries by skill intensity

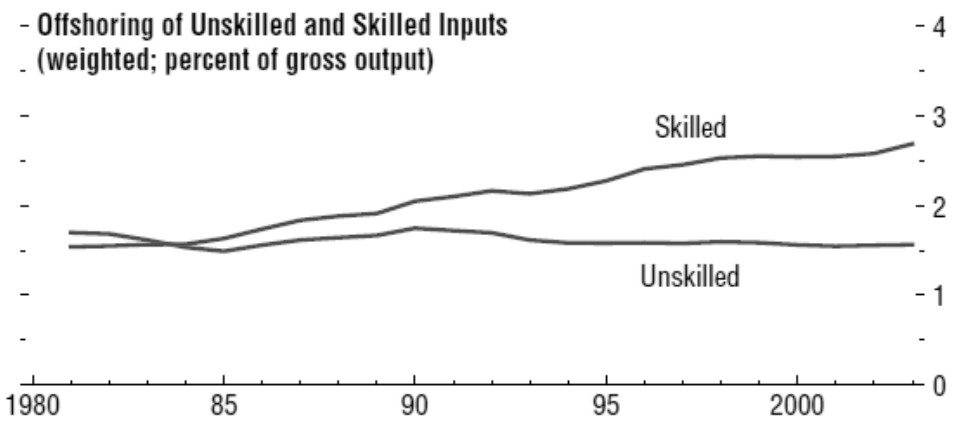

Source: (IMF, 2007, Figure 5.6). Notes: Advanced countries include Austria, Canada,

France, Germany, Japan, the Netherlands, the United Kingdom, and the United States.

of the offshoring of service activities, whereby the more routine service activities are sent overseas" (Feenstra, 2008, p.3). To explain this new form of offshoring, it is therefore necessary to elaborate on changes in the wage structure by studying the characteristics of occupations in more detail.

Figure 1.8: Relative Employment of Nonproduction/Production Workers in the U.S. Manufacturing

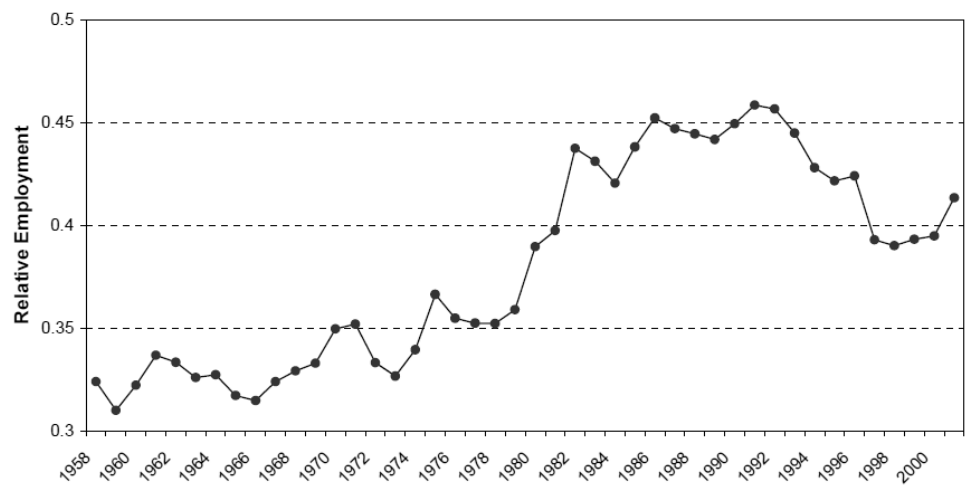

Source: (Feenstra, 2008, Figure 1.2).

More interestingly, data from the U.S. labor market show that the last four decades have been characterized by two contrasting periods of wage structure changes. The first period, 1970s through the late 1980s, was associated with a monotone expansion of wage inequality in the top and bottom halve of the wage distribution, while the second period, starting from the early 1990s, shows a non-monotone relationship of wage inequality, i.e. a simultaneous increase in the wage growth of high and low wages relative to the middle (see Acemoglu and Autor, 2011, for a thorough survey). These contrasting wage trends coincide with recent changes in the occupational structure. Figure 1.9 depicts changes in the share of employment decomposed into three sets of occupations grouped according to average wage level (low, medium, and high) in each of $16 \mathrm{EU}$ countries and the U.S. during 
Figure 1.9: Changes in employment shares by occupation 1993-2006 in 16 European countries and the USA

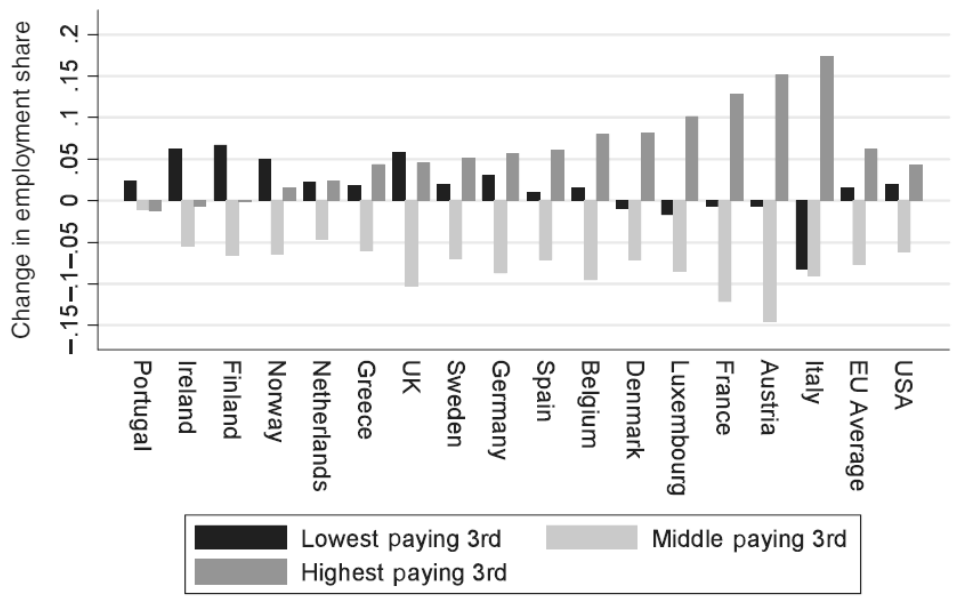

Source: (Acemoglu and Autor, 2011, Figure 11). Notes: Occupations are grouped to three broad categories: low, medium and high, by wage levels.

the period 1993 through 2006. It is readily evident that the middle wage occupations have substantially declined in all sample countries, where the most pronounced changes have occurred in Austria, France and the UK by 14, 12, and 10 percentage points, respectively (cf. Acemoglu and Autor, 2011). Remarkably, this substantial decrease in the "middle skill" occupations has been offset by a rapid growth in both low and high wage occupations. This pattern reflects another stylized fact, referred to as the polarization of the labor market.

The rationale for the polarization of labor market has been put forth by advances in technology, particularly in information and communication technology (ICT), enabling many firms to bundle occupational tasks, that exhibit a high content of rule-based, routine activities, and codify them in computer software (cf. Autor et al., 2003). These routine tasks are characteristic of many middle skilled occupations, such as bookkeeping, clerical work, repetitive production, and monitoring jobs (cf. Acemoglu and Autor, 2011). Simultaneously, these advances in technology and logistic systems have substantially lowered the cost of offshoring, creating incentives for firms to reorganize the production and work process by sending electronically these routine, non-interactive tasks abroad (cf. Blinder, 2009a) - a new development that Snower et al. (2009) call the "Great Reorganization" era. ${ }^{6}$

Similar to the discussion in the context of immigration and skill downgrading, this offshoring-induced displacement effect may have indirect implications for low-skilled workers. Stated differently, medium-skilled workers, who were initially displaced due to offshoring, may be reabsorbed by the labor market by reallocation into low-skill-intensive occupations. Indeed, Autor and Dorn (2013) find empirical evidence from the U.S. that

\footnotetext{
${ }^{6}$ Yet, there is no clear consensus among economists regarding which of the two factors (ICT vs outsourcing) is the important driving force behind the recent polarization wage trends, see Feenstra (2008) for a discussion.
} 
polarization of employment was associated with a substantial increase in low-skill service jobs.

Thus, a natural reaction to increasing offshoring activities of these "good" jobs in the advanced countries would go along the lines of the following argument

Hypothesis 2. Offshoring induces a reallocation of domestic jobs performed mostly by medium-skilled workers in the advanced countries, lowering their wages and employment opportunities. This in turn will raise the competition for low-skill jobs and eventually lead to a crowding-out of low-skilled workers from the labor market.

As outlined below, chapter 3 is devoted to this argument and elaborates analytically the determinants of and the interaction between various potential driving forces behind the labor market impact of offshoring. As discussed below, offshoring induces a costenhancing effect that may raise the real wages of all workers and lead to a decline in the low-skill unemployment rate.

Despite the concerns associated with these trends, two strands in the literature provide alternative explanations regarding potential adjustment channels that direct and absorb the shocks of immigration and offshoring in the receiving economy. One set of studies has emphasized the role of endogenous technological change (cf. Acemoglu, 1998, 2002b; Acemoglu et al., 2012; Autor et al., 1998; Katz and Murphy, 1992). The idea behind this adjustment mechanism is that changes in the skill endowment lead to an endogenous adjustment in skill-complementary technology. The vast empirical studies have established a wide consensus that the pervasive wage inequality, documented throughout the 1970s, 1980s and 1990s in the U.S., was primarily driven by a strong growth in relative labor demand for skilled workers due to skilled-biased technological changes (SBTC) (cf. Lemieux, 2008). Thus, this view relates the bias in technological changes to the substitutability between skilled and unskilled labor.

Another set of studies provide a competing interpretation by emphasizing the endogenous choice of production technologies, e.g. modern versus traditional machines (cf. Beaudry et al., 2010; Beaudry and Green, 2003; Caselli, 1999). The core argument of this view is marked by the substitutability between technologies instead of labor, where the adoption is a costly endogenous process (cf. Caselli, 1999). Thus, faster adoption of technologies, according to this view, will mitigate the direct implications of increasing competition caused by a labor supply shock (cf. Beaudry et al., 2010).

Summarizing, the next matter of concern regarding the labor market impact of immigration can be described by

Hypothesis 3. Changes in the skill structure induced by immigration will affect the structure of the production technology mode in the economy and thus influence the wage structure of native workers.

Chapter 4 focuses on this issue and provides an analytical discussion regarding the determinants of and interaction between different adjustment mechanisms. As elaborated below, immigration-induced changes in the skill mix of the receiving countries leads to higher adoption of the complementing production techniques (a technology adoption effect) that counteracts the direct adverse labor supply effect.

Finally, a large body of empirical literature has addressed the concerns regarding the 
extent of substitutability/complementarity between immigrants and natives (see among many others Borjas, 2003; Brücker et al., 2014; D'Amuri et al., 2010; Manacorda et al., 2011; Ottaviano and Peri, 2008). Using what Acemoglu and Autor (2011) refer to as the canonical approach, i.e. the decomposition of labor into skills, nationality and experience cells and nesting each cell through a Constant Elasticity of Substitution (CES) aggregate, the common consensus from these studies is that immigrants are closely substitutes for other immigrant groups, but demonstrate a substantial degree of imperfect substitutability to natives. The intuitive explanation for these findings follows from the differential comparative advantages between immigrants and natives. Recent empirical evidence has documented that immigrants perform mainly occupations that are characterized by a high content of manual tasks, while natives tend to specialize in occupations with a high content of communication and complex tasks (cf. Ottaviano et al., 2013; Peri and Sparber, 2009). Moreover, recent empirical studies show that immigration induces firms to use intensively factors (labor) that are complement to immigrants (cf. Dustmann and Glitz, 2012; González and Ortega, 2011). As mentioned above, offshoring also induces substantial changes in the occupational structure of the domestic labor market, leading to changes in the production process in the domestic economy.

Summarizing, the following argument describes the concerns regarding the implications of task reallocation

Hypothesis 4. Immigration and offshoring induce substantial changes in the structure of tasks and occupations, respectively, and thus lead to adverse labor market outcomes for native workers due to specialization of firms in production techniques complementing immigrants and offshoring.

Addressing this issue is the objective of chapter 5, which examines analytically the distributional implications of changes in the task and occupational structure induced by immigration and offshoring, respectively, and compares their underlying driving forces affecting the firm's decision regarding adoption of production technologies. As will be outlined below, the analysis reveals that both immigration of medium-skilled workers and offshoring of medium skill-intensive jobs induce a wage polarization effect, whenever the initial level of immigrants' task share and offshoring rate are high.

\subsection{Outline of the thesis}

These four hypotheses constitute the core objective of this thesis. The remaining chapters are organized as follows.

\section{Chapter 2: Migration, unemployment, and skill downgrading}

To address the concerns described in Hypothesis 1, an integrated general equilibrium framework is developed that accounts for important features such as low-skill unemployment and skill downgrading of medium-skilled workers. More precisely, the economy is described by a two-sector (manufacturing and service) specific factors model consisting of monopolistic competition and skill heterogeneity. The labor market is characterized by three skill groups (low, medium and high) where low- and medium-skilled 
labor markets exhibit frictions due to a right-to-manage collective bargaining. Workers are allocated across the two sectors, where low-skilled and medium-skilled workers are employed only in the service and manufacturing sector, respectively, while high-skilled are perfectly mobile between the two sectors.

This chapter contributes to several strands in the literature addressing the wage and employment effects of immigration (cf. Brücker and Jahn, 2011; D'Amuri et al., 2010), and indirect adjustment channels absorbing immigration shock such as changes in the output mix and goods price (cf. Felbermayr and Kohler, 2007) in two important respects. First, the core focus of the analysis in this chapter is to examine the impact of immigration on skill downgrading and low-skilled unemployment. Second, particular attention is paid on the role of the labor market institution, which is captured by two distinct labor market regimes: a flexible labor market, characterized by an endogenous unemployment benefit scheme, and a rigid labor market, with an exogenous unemployment benefit scheme.

The analysis reveals two interesting insights. First, immigration-induced increase in the downgrading rate generates a complementarity effect, leading to a decline in the lowskilled unemployment rate under the flexible labor market regime. Second, the numerical simulation of recent trends of skilled workers quantifies the potential welfare effects of immigration, leading to gains in GDP per capita between 6 and 10 percent.

\section{Chapter 3: Offshoring of medium-skill jobs, polarization, and productivity effect: Implications for wages and low-skill unemployment}

This chapter addresses the concerns captured by Hypothesis 2 by investigating analytically the impact of offshoring on the wage and employment structure of the offshoring source country. An integrated theoretical framework is developed that accounts explicitly for several important features: task-skill heterogeneity (low, medium and high), endogenous offshoring, and spill-over effects across skill groups due to mobility across job tasks. Thus, this model integrates several features from the existing theoretical contributions, incorporating explicit assignment of skills to tasks (cf. Acemoglu and Autor, 2011) and allowing for an endogenous relocation process of offshoring tasks (cf. Grossman and Rossi-Hansberg, 2008; Ottaviano et al., 2013) into a richer framework that allows for equilibrium unemployment of low-skilled workers. More precisely, we allow for two types of labor market frictions. The first is given by a minimum wage scheme, while in a second step we introduce a more general notion of labor market adjustments by allowing for endogenous low-skilled labor supply.

The core objective of this chapter is twofold. First, we analyze the impact of offshoring jobs on changes in the wage structure of domestic workers. In doing so, we show the distributional effects of different scenarios of offshoring incidence, where the burden of job cuts is born by different skill groups. In a second step, we analyze the spill-over effects of offshoring-induced reallocation of medium-skilled workers into low-skill jobs on low-skill unemployment.

The analysis provides several new insights. First, offshoring of low skill-intensive job tasks generates distributional effects similar to "skill-biased" technology changes (cf. Acemoglu, 2002b), offshoring of medium skill-intensive tasks leads to wage polarization (capturing 
recent trends), while offshoring of high skill-intensive tasks induces wage effects similar to "unskilled-biased" technological changes (cf. Goldin and Katz, 2009). Second, we show that a marginal decline in offshoring costs generates two interacting forces. On the one hand, there is a displacement effect of domestic workers due to relocation of tasks. There is, on the other hand, a productivity effect due to cost-efficiency improvements. Due to endogenous task reallocation across the skill groups, the productivity gains accruing to workers, who were initially displaced by offshoring, are mitigated. The rationale behind this result, put forth by the empirical literature, is given by losses in task-specific human capital and search costs (cf. Hummels et al., 2014). Finally, we show that the spill-over effect on low-skilled unemployment of medium-skilled workers due to offshoring-induced bumping down into low skill-intensive tasks is crucially determined by the degree of substitutability between low and medium skill-intensive tasks.

\section{Chapter 4: Immigration, technology adoption and wage inequality}

This chapter addresses the concerns regarding the impact of immigration on changes of the structure and of the direction of technology (Hypothesis 3). A theoretical model is developed that for a richer setup of the goods and the labor market. More precisely, the model economy is characterized by firm heterogeneity, endogenous technology adoption, monopolistic competition and skill heterogeneity (low, medium, and high). A distinct range of final goods is produced by one of the three technology modes, where the technology allocation is defined by two endogenously determined cutoff points. Each technology mode is complementing one specific skill group - capturing the notation of capital-skill complementarity (cf. Acemoglu, 1998). The Walrasian nature of the labor market permits to pay the attention primary to changes in the wage structure of native workers. Moreover, the economy is characterized by increasing returns to scale, which is induced by endogenous changes in the mass of monopolists, allowing to capture the notation of directed technological changes (cf. Acemoglu, 2002b).

The objective of this chapter is to examine how immigration-induced changes in the skill mix affects the nature of the technology adoption behavior of firms and what are the consequence of changes in the structure of the production technology for the wage structure. The analysis reveals two new insights. First, immigration-induced changes in the wage structure are characterized by two interacting effects: a direct market size effect, referring to an increase in the skill endowment, and a technology adoption effect, referring to endogenous changes in the technology cutoff points. The former effect has usually an adverse wage impact for domestic workers hit by immigration due to higher labor market competition, while the latter raises the wages of those workers as the economy becomes more specialized in the complementing technologies. Moreover, the results reveal that the relative magnitude between these two effects is importantly determined by the degree of technology adoption, reconciling the empirical evidence (cf. Beaudry et al., 2010), while the direction of each of two effects depends on the interaction between consumer preference (final goods demand) and the market power of monopolists (i.e. the substitute ability between intermediate goods). 


\section{Chapter 5: Immigration, offshoring, tasks and technology adoption: Implications for native wage structure}

This chapter addresses the concerns regarding the implications of immigration-induced changes in the organizational structure for the wage structure of native workers (Hypothesis 4). In this chapter, the theoretical framework is built on the model developed in chapter 4, and augments that framework with respect to two important aspects. First, it allows for internal work organization adjustments, associated with task allocation between immigrants and natives, where according to the observations immigrants are allocated to manual-intensive tasks and natives to communication-intensive tasks (cf. Peri and Sparber, 2009). Second, it introduces offshoring, whereby a fraction of mostly medium-skill intensive domestic jobs (intermediates), as documented in the literature, are relocated and produced abroad (cf. Acemoglu and Autor, 2011).

The analysis reveals several new insights. First, immigration and offshoring induced two interacting effect: an efficiency effect and a labor supply effect. Second, the relative strength of efficiency effect induced by immigration (through task-reallocation) and offshoring (through job reallocation) depends crucially on the initial levels of tasks allocated to immigrants and offshoring rate. At low initial levels the efficiency becomes the dominating force, leading to higher wages of native workers of the same skill type. Moreover, the direction of and relative magnitude between the technology adoption effect and the market size effect are consistent with the discussions in chapter 4 . However, the comparative statics highlight the following interesting results. Whenever the substitutability between different technology modes becomes extremely easy, such that the technology adoption effect and the market size effect are balanced, the efficiency gains due to task-reallocation and cost-savings from offshoring still remain. This result highlights the crucial departure from recent immigration and offshoring literature (cf. Acemoglu et al., 2012; Ottaviano et al., 2013).

Thus, these results highlight the important adjustments at the macro level, referring to technology adoption effect, as well as at the micro level, referring to reallocation of tasks and jobs. 


\section{Migration, Unemployment, and Skill Downgrading ${ }^{1}$}

\subsection{Introduction}

The migration pattern in Europe has significantly changed over the last decades, driven by the integration of national markets into global markets as well as the soaring demand for better educated, high-skilled workers due to an intensified international competition. In general, the overall stock of immigrants with higher educational attainments has increased significantly. For instance, the East-West immigration patterns in the course of the enlargement of the EU in the past decades have seen a substantial increase in the supply of medium-skilled immigrants (i.e., with upper secondary education). ${ }^{2}$

On the other hand, looking at the labor market outcomes of immigrants, one considerable friction becomes evident. Immigrants face a substantial risk of job-skill mismatches. Several recent studies have found that immigrants with higher educational levels, especially those who moved in the course of the Eastern EU-enlargement, have been relegated to jobs which require a lower educational/skill attainment - indicating a skill downgrading in occupations. ${ }^{3}$ In an empirical cross-country analysis, Brynin and Longhi

\footnotetext{
${ }^{1}$ This chapter is based on joint work with Joan Muysken and Thomas Ziesemer, and is published in Scandinavian Journal of Economics (Muysken et al., 2015). We are grateful to Herbert Brücker, Caglar Özden, Klaus Prettner, and two anonymous referees for valuable comments and discussions, to Stella Capuano for providing data on immigration and education, and to participants at NAKE Research Day 2011 in Utrecht, at 17th SMYE in Mannheim, and at EALE 2012 in Bonn.

${ }^{2}$ This refers to the entry of eight Central Eastern European countries (CEECs) and two Mediterranean countries into the EU on May 2004. See Kahanec and Zimmermann (2010) for a survey of recent migration patterns in the EU and Blanchflower et al. (2007) for recent patterns in the UK.

${ }^{3}$ See Drinkwater et al. (2009); Dustmann et al. (2013) for recent evidence in the UK. For example, Dustmann et al. (2013) empirically assess the immigration effect along the wage distribution. Although the newly arrived immigrants to the UK have on average higher educational attainments, the authors find that they are located at the lower end of the wage distribution- evidencing a skill downgrading effect. For cross-country evidence, see OECD (2007b).
} 
(2009) find that the incidence of skill downgrading is more pronounced among workers with intermediate skills.

In sum, these observations emphasize on the one hand an immigration-induced shift in the labor supply of better educated workers over the last decade. On the other hand, the immigration-driven rise in skill downgrading offers a rationale for potential displacement effects of the least skilled native workers. ${ }^{4}$ The objective of this chapter is to revisit the labor market effects of immigration by assessing the impact of immigration on skill downgrading and unemployment in a general equilibrium framework.

Intuitively, higher skill downgrading due to the immigration of better educated workers should induce a substitution effect for the least skilled workers, thus displacing them from the labor market. However, as we elaborate below, the labor market institutions play an important role in determining the impact of immigration on low-skilled unemployed workers. Particularly, under a flexible labor market regime, an immigration-induced rise in skill downgrading generates a decline in the low-skilled unemployment rate indicating a complementarity effect. The reason is that in a flexible labor market regime, the reservation wage of unemployed workers is usually low and much more responsive to economic shocks compared to a more rigid labor market.

As a consequence of skill downgrading, immigration of medium-skilled workers can generate an expansionary effect of low paid service jobs which, in turn, induces a wage polarization effect, i.e. a decline of medium-skilled wages relative to low- and high-skilled wages, a similar effect to technology advances. To our knowledge, this is a new insight which has not been addressed in the literature. Whereas high-skilled migration is widely accepted and low-skilled migration is mostly reduced by current policies, medium-skilled migration is discussed very little in politics and academia.

To offer an integrated explanation of the above observations and include the mediumskilled migration in the analysis, we develop a two-sector ("manufacturing" and "services") model with heterogeneous workers. ${ }^{5}$ We assume that low-skilled workers are employed in the services sector whereas medium-skilled workers are employed mainly in the manufacturing sector. Finally, a common and perfectly mobile factor, such as high-skilled labor, is employed in both sectors. In line with the institutional labor market setting in many European countries, we assume a standard collective bargaining approach: a right-to-manage bargaining model. We also assume that medium-skilled workers who do not find a job in the manufacturing sector have the outside option to take a low paid job in the services sector, while low-skilled workers, who find no service jobs, end up in unemployment. ${ }^{6}$ We capture the nature of flexibility of the labor

\footnotetext{
${ }^{4}$ Several recent studies have shown that, in general, attitudes towards immigration are heterogeneous across native population and depend on the labor market situations, welfare considerations, and noneconomic factors (see, for example, Dustmann et al., 2008; Dustmann and Preston, 2007; Facchini and Mayda, 2008; Mayda, 2006). See also Boeri and Brücker (2005) for a discussion of concerns regarding "welfare shopping".

${ }^{5}$ It is worth mentioning that a more general framework would also consider within sector firm heterogeneity, which permits to account for within-sector occupation wage gap (cf. Helpman et al., 2014). Our intention is, however, to address another recently observed phenomenon reflecting wage inequality between the occupations (or between skill groups), the so called polarization effect, which might partly be induced by labor supply shocks.

${ }^{6}$ Notice that in doing so we ignore the importance of individual job search behavior, such as on-the-job
} 
market by endogenizing the unemployment benefits of displaced low-skilled workers. Our model enables us to address different adjustment channels, such as shifts in relative labor demand, considering the "substitution effect" between different input factors, as well as shifts in the labor supply, taking the "crowding-out" effect under different labor market regimes into account.

Finally, we examine numerically the general equilibrium effects of an infra-marginal increase (i.e., a discontinuous jump) in the labor force due to immigration both for flexible and rigid labor market regimes. In general, our numerical analysis generates two interesting predictions:

- In a flexible labor market, i.e. with an endogenous unemployment benefit scheme, immigration of medium-skilled workers generates a complementarity effect, boosting employment of low-skilled workers, while in a rigid labor market, i.e. with exogenous unemployment benefits, medium-skill immigration induces a substitution effect, displacing low-skilled workers. ${ }^{7}$

- The differences in the welfare effects of various migration patterns are substantial, where the recent immigration trends of educated workers increase the GDP per capita between 6 to $10 \%$.

The set up of the chapter is as follows. Section 2.2 reviews the existing and related literature followed in Section 2.3 by the presentation of the stylized facts on migration patterns, the relation between low-skilled unemployment and the skill downgrading of medium-skilled workers, and trends in employment for the major Wester European destination countries. In Section 2.4 we exposit the theoretical framework with two major sectors, three skill groups, and a double wage bargaining model determining the wages of medium- and low-skilled labor. In Section 2.5, we provide a qualitative assessment of a comparative static analysis for two different labor market regimes. In Section 2.6, we calibrate the model for two countries (France and Germany) representing different labor market institutions using the EUKLEMS data set to measure the quantitative importance of various migration scenarios. Finally, Section 2.7 presents some concluding remarks.

\section{$2.2 \quad$ Literature review}

In the growing literature on economic and labor market effects of migration the analysis in this chapter is related and contributes to various strands in the literature.

\subsubsection{Employment and wage effects: the canonical approach}

There exists a vast literature examining various channels of immigration-induced economic and distributional effects on the host country. One strand of the literature

search for medium-skilled workers which reflects the trade-off between being unemployed or staying in low paid service jobs and searching for job opportunities in the manufacturing sector.

${ }^{7}$ See also Beladi (1990), who shows that the accumulation of a specific factor can increase the total employment in an unemployment-plagued economy. However, he neither incorporates manufacturing price effects nor a heterogeneous labor force. 
focuses on labor market effects through adjustment channels such as changes in wages and (un)employment through which native workers are potentially directly affected. ${ }^{8}$ In spite of an overall negligible effect of immigration on wages and employment, the findings of these studies, using the canonical (multi-nested production technology) model, emphasize the role of factors such as changes in the skill structure, substitutability between natives and immigrants, and the elasticity of the capital supply. For example, the easier the substitutability becomes, the larger the burden is for native workers, while a perfectly elastic capital supply induces the so-called "immigration surplus" (Borjas, 1995; Dustmann et al., 2008). ${ }^{9}$ However, these studies assume implicitly that all newly arrived immigrants are perfectly integrated in the labor market according to their skill level, i.e., no skill-job mismatch. We address this omission and supplement this strand of the literature by assessing the impact of immigration on skill downgrading and unemployment. Furthermore, we elaborate the interaction between these two friction variables together with sectoral composition effects driven by endogenous manufacturing prices under different labor market institutional regimes.

\subsubsection{Absorption channels of immigrants in the economy}

Another strand in the literature emphasizes different (indirect) adjustment mechanisms such as changes in the output mix (sectoral composition) and manufacturing prices through which immigrants are absorbed by the host economy. ${ }^{10}$ The overall conclusion is that in a small open economy where manufacturing prices are determined by international markets, immigration flows generate a Rybczynski-type of effect, indicating the insensitivity of wages to immigration. In particular, the recent empirical evidence, focusing on local industry-labor markets, highlights that immigration induces a technology or intensity effect, i.e., an increase in the relative use of the enhanced factor in the production process, and to some extent a scale effect, i.e., a relative expansion of output/firms using immigrants (or factors with similar characteristics) more intensively (Dustmann and Glitz, 2012; González and Ortega, 2011; Lewis, 2003). ${ }^{11}$ We contribute to this literature by incorporating simultaneously these different adjustment mechanisms when examining the impact of immigration on skill downgrading and unemployment under different benefit scheme regimes.

\footnotetext{
${ }^{8}$ Important early contributions are Borjas (1995); Borjas et al. (1996); Card (1990) as well as recent contributions by Borjas (2003); Ottaviano and Peri (2008, 2012) for the US; recent important studies on the German labor market are Brücker and Jahn (2011); D'Amuri et al. (2010); Dustmann et al. (2009); Felbermayr et al. (2010); on the UK labor market Dustmann et al. (2005, 2008); Manacorda et al. (2011); as well as the cross-country evidence for UK, Germany and Denmark by Brücker et al. (2014).

${ }^{9}$ The main conclusion of these recent studies is that natives and immigrants are imperfect substitutes and that newly arrived immigrants compete mostly with incumbent immigrants rather than with native workers with similar characteristics.

${ }^{10}$ See recent contributions by Cortes (2008); Dustmann and Glitz (2012); Felbermayr and Kohler (2006, 2007); González and Ortega (2011); Lewis (2003).

${ }^{11}$ Dustmann and Glitz (2012) emphasize further the role of newly created firms, explaining 18 percent of the overall adjustment to migration-induced labor supply shocks. The authors also find significant negative wage effects for the non-traded sector.
} 


\subsubsection{Advances in technology and the polarization of the labor market}

A recent development that has received a lot of attention emphasizes substantial changes in the structure of occupations in many advanced countries. This observation reveals a significant increase in low paid service and highly-skilled jobs over the last decade indicating a shift in the labor demand against medium-skill intensive occupations. The literature examining the driving force behind this phenomenon emphasizes the role of progress in information and communication technology (ICT), replacing workers by machines and computers, as the main driving force behind this development. ${ }^{12} \mathrm{We}$ provide another possible driving force behind this effect. Due to the incidence of skill downgrading, a labor supply shock induced by immigration of medium-skilled workers can generate a similar polarization effect. As emphasized by Autor and Dorn (2013), consumer preferences play an important role in explaining this rise in service jobs. If consumers favor variety over specialization in consumption, then an unbalanced-biased technology progress raises the aggregate demand for service output - a phenomenon also known as the Baumol (1967)'s disease. ${ }^{13}$

We incorporate this implication in our general equilibrium framework but with two distinct differences from Autor and Dorn (2013). First, in our framework, the labor market is characterized by frictions where medium-skilled workers face the risk of being downgraded into low-paid, service jobs, while low-skilled workers end up in unemployment. Second, assuming a more general functional form in the service sector, we show that the rise in the demand for aggregate service output is not necessarily associated with higher demand for labor in that sector. In fact, it depends on the interaction between consumer preferences, i.e., the substitutability between different consumption goods, and production technology, i.e., the substitutability between input factors in the production process, especially in the service sector.

\subsubsection{Transferability of human capital and the assimilation of immigrants}

Our framework is also related to the strand of literature addressing the determinants of the assimilation process of immigrants in the host country. The empirical findings reveal that, upon arrival, immigrants on average earn considerably less than comparable natives, but over time, they catch up with natives (cf. Chiswick, 1978). One of the main reasons is that the education and work experience obtained abroad are substantially downgraded in the host country (see, for example, Chiswick and Miller, 2009, 2012; Friedberg, 2000). We capture the nature of initial skill-downgrading by allowing the average medium-skill

\footnotetext{
${ }^{12}$ This phenomenon is also known as the "polarization" phenomenon, implying a relative change against occupation at the middle range of the skill distribution that can be easily computerized. See Acemoglu and Autor (2011) for a survey of recent important contributions.

${ }^{13}$ Generally, the hypothesis claims that unbalanced technical progress in sectors with high TFP growth, such as manufacturing, will also induce a rise in employment/wages in sectors with low TFP growth, like services.
} 
over-qualification rate to vary with immigration. ${ }^{14}$ Our intention is neither to model the dynamic assimilation process itself nor to explain its determinants regarding the individual labor market performance. In fact, we take a different step and pay attention to the labor market consequences of initial downgrading for other labor force participants. In so doing, we are able to gain new insights from migration-induced job-skill-mismatch and the implications for other skill groups in the host country. So far, this channel has received less attention in the literature and the analysis in this chapter fills this gap.

\subsubsection{Brain drain, gain, and waste: The perspective of the sending countries}

In our model migrants were classified as high, medium or low-skilled according to their position in the production function. Many of them would be classified differently, mostly more skilled, in their countries of origin. This phenomenon is called brain waste, because it indicates that migrants could work in higher skilled jobs, if the skills were perfectly equivalent and transferable. The reasons for this waste are differences in quality of education - due to low expenditure on tertiary education - and English language (like those for visa and family re-union targeting more or less the specific scarce qualifications) (Mattoo, 2008; Özden, 2006). Of course the brain waste is largest during times that immigrants are unemployed. Nevertheless, on average more highly educated migrants earn more in the country of destination. These earnings feed a flow of remittances to the countries of origin compensating at least partly for the brain drain that happened to occur in the first instance (unless emigrants were unemployed before their migration; Bhagwati and Rodriguez, 1975).

The remittances and the higher success of more educated migrants signals that education may be highly profitable and prestigious. This may lead to higher enrolments by families with children, often financially supported by the diaspora, and more public expenditure on education from the government (Ziesemer, 2012). If this leads to higher education than without preceding brain drain the literature speaks of a brain gain (Docquier, 2006), requiring that many stay in the country and are employed without much over-education. There are now many studies of the brain drain and gain considering a great diversity of sectors, countries and skills with mixed evidence (Docquier and Rapoport, 2012). One interesting result from them is that remittances roughly cover the fiscal costs of the brain drain (Gibson and McKenzie, 2011) although they go to private persons. Finally, some migrants return and bring back knowledge for improving their countries, especially if they have been successful.

In countries of origin the success of migration depends also on adequate complementary education policies (see Docquier and Rapoport, 2012; Garcia Pires, 2009; Ratha et al., 2011). For the countries of destination, making migration a success in developing countries depends very much on complementary policy actions. In our model these refer to the nature of benefit schemes, which determine whether or not medium-skilled migration reduces low-skilled unemployment and increases GDP and future pensions.

\footnotetext{
${ }^{14}$ As the downgrading also happens to occur to non-migrant workers after job loss (Autor et al., 2013), we do not have to distinguish between downgrading of migrants and non-migrants.
} 


\subsection{Stylized facts}

As mentioned above, over the last two decades there has been a substantial improvement in the educational attainments of immigrants: a shift towards the middle and upper range of the skill distribution. Table 2.1 highlights this feature for the major Western European destination countries. It is noticeable that over the last two decades in all countries the share of the immigrant population with lower educational attainments has considerably declined.

Table 2.1: Educational attainments of immigrants in selected countries of destination

\begin{tabular}{|c|c|c|c|c|c|c|}
\hline \multirow[b]{2}{*}{ Country } & \multicolumn{3}{|c|}{ Shares in 2010 (in \%) } & \multicolumn{3}{|c|}{$\begin{array}{c}\text { Percentage changes } \\
\quad 1995-2010\end{array}$} \\
\hline & Low & Medium & High & Low & Medium & High \\
\hline \multicolumn{7}{|c|}{ All Immigrants } \\
\hline Austria & 37.4 & 45.9 & 16.7 & -13.2 & 3.5 & 33.0 \\
\hline Denmark & 49.2 & 28.8 & 21.9 & -9.9 & 6.9 & 13.2 \\
\hline France & 80.5 & 7.5 & 11.9 & -20.1 & 73.2 & 89.5 \\
\hline Germany & 53.1 & 30.5 & 16.4 & -17.5 & 13.1 & 32.3 \\
\hline Ireland & 45.0 & 21.0 & 34.0 & -42.0 & 14.8 & 46.4 \\
\hline Netherlands & 63.2 & 19.5 & 17.3 & -28.8 & 50.1 & 48.7 \\
\hline Spain & 32.7 & 46.3 & 21.0 & -41.2 & 18.3 & 23.8 \\
\hline Sweden & 43.4 & 36.1 & 20.5 & -27.4 & -2.3 & 62.2 \\
\hline UK & 57.1 & 16.9 & 26.0 & -23.9 & -54.9 & 88.3 \\
\hline \multicolumn{7}{|c|}{ Immigrants from New Member States } \\
\hline Austria & 24.9 & 56.1 & 19.0 & -6.1 & -3.1 & 22.0 \\
\hline Denmark & 53.7 & 25.7 & 20.5 & 27.1 & -22.2 & -16.6 \\
\hline France & 46.1 & 20.9 & 33.0 & -38.7 & 91.2 & 136.6 \\
\hline Germany & 21.7 & 49.1 & 29.2 & -15.2 & 3.4 & 8.4 \\
\hline Ireland & 31.9 & 34.2 & 34.0 & -13.9 & 45.9 & -14.2 \\
\hline Netherlands & 44.9 & 29.4 & 25.8 & -29.0 & 50.4 & 49.3 \\
\hline Spain & 19.2 & 54.7 & 26.1 & -41.2 & 18.3 & 23.8 \\
\hline Sweden & 24.4 & 38.2 & 37.5 & -29.9 & 0.9 & 36.5 \\
\hline UK & 51.5 & 9.2 & 39.3 & -9.8 & -45.9 & 51.4 \\
\hline
\end{tabular}

Source: Brücker et al. (2013).

Note: The educational levels are defined as follows: Low = ISCED 0-2, Medium = ISCED 3-4, High = ISCED 5-6. The data denotes the shares in immigrant population above 25 years. The New Member States are EU-10 countries: Cyprus, the Czech Republic, Estonia, Hungary, Latvia, Lithuania, Malta, Poland, Slovakia, and Slovenia.

Moreover, the educational attainments of immigrants from the New Member States (NMS) joining the European Union in 2004 possess a similar pattern, constituting a supply shock of better educated workers. Finally, medium-skilled workers make up a major part of the immigrants and the native labor force in many European, see Brücker et al. (2014) for a cross-country evidence.

It is documented in OECD (2007b) that the labor market performance of immigrants across many advanced countries has stronger mismatches than do natives, indicating a higher incidence of skill downgrading. Empirical studies reveal that a significant and increasing proportion of low-skilled jobs are nowadays carried out by better educated, overqualified workers - see Borghans and de Grip (2000) and Hartog (2000) for an overview of these studies. Recent studies on post EU-enlargement have provided further evidence. For example, Drinkwater et al. (2009) analyze the performance of Polish immigrants in the UK labor market and find that the majority of them are employed in 
Figure 2.1: Net replacement rate and regression coefficients of low-skilled unemployment versus medium-skilled over-qualification rates

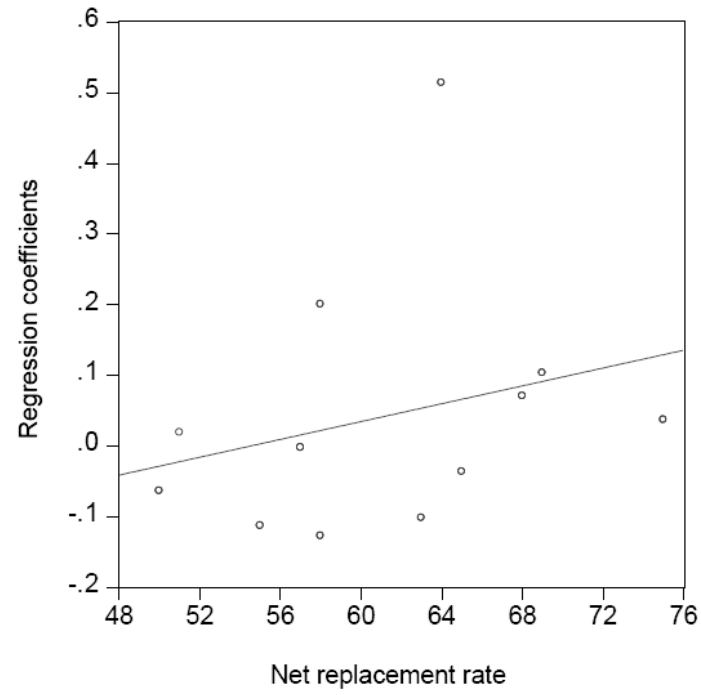

Notes: For the estimated coefficient see 2.A.3, Table 2.6. The regression coefficients are presented in 2.A.2, Table 2.5, column 4. The net replacement rate includes social assistance and housing benefits, see 2.A.1 for further details.

low-skilled and low paid jobs despite having relatively high levels of education. Moreover, Brynin and Longhi (2009) find an excess of skill downgrading at the medium-skilled level (i.e., with upper secondary schooling) which contributes almost one-half of all overqualified persons.

These stylized facts suggest that the rise in low-skilled unemployment would not only be the result of a relative demand shift driven by technology progress, but also the consequence of a relative supply shift which leads to a crowding out of low-skilled workers, as has also been observed by Pierrard and Sneessens (2003).

In order to verify the latter notion of crowding out, we have regressed the low-skilled unemployment rate to the skill downgrading rate of medium-skilled workers, next to other control variables, for 14 European countries for which data were available - details are presented in 2.A.2. From these regressions we found that for one group of countries - Denmark, France, Greece, Ireland, Italy, the Netherlands and Sweden - down grading of medium-skilled workers indeed has a positive impact on low-skilled unemployment. However, for another group of countries Austria, Germany, Belgium and Finland the impact of down grading of medium-skilled workers on low-skilled unemployment turns out to be negative, surprisingly enough. ${ }^{15}$

We believe that this difference can partly be explained by changes in the labor market institutions with the objective of making the labor market more flexible, especially for low-skilled workers OECD (2013). The first group of countries then has an inflexible labor market which prevents wage adjustments to absorb bumped-down low-skilled

\footnotetext{
${ }^{15}$ For Portugal, Spain and the UK the impact was not significantly different from zero.
} 
workers, resulting in low-skilled unemployment. In the second group of countries wage adjustments prevent low-skilled unemployment. Taking the net replacement rate (NRR) as an indicator for flexibility, this is consistent with the observation that the first group has on average a replacement rate of 67 percent while the second group has an average replacement rate of 60 percent. ${ }^{16}$

Moreover, the regression coefficients between low-skilled unemployment and mediumskilled over-qualification rates (see 2.A.2) show a weak but significantly positive relationship to the net replacement rates (NRR), which strengthens our perception that labor market institutions have an impact on the adjustment of low-skilled unemployment to bumping down - see Figure 2.1. ${ }^{17}$

Finally, as mentioned earlier, the recent empirical evidence verifies a contraction of medium skill intensive occupations in many advanced countries - cf. Goos et al. (2011) for recent evidence in Europe. Interestingly, looking at the disaggregated skill-occupation level, the share of low- and medium-skilled workers employed in the lowest paying occupations, mostly services, has substantially increased over the last decades. Table 2.2 exhibits this trend and highlights the skill downgrading of medium-skilled workers. We summarize these stylized facts as follows.

1. The proportion of highly educated immigrants has substantially increased in many European countries over the last years, still medium skills constitute the majority of immigrants labor force.

2. Both low- and medium-skilled employment has strongly risen in low paid service jobs.

3. There has been a continuous downgrading of medium-skilled labor.

4. The relation between skill downgrading and low-skilled unemployment rates can be partly explained by changes in labor market institutions, of which the replacement rate is an indicator.

Based on these stylized facts, we develop in the next section a theoretical framework that permits a structured analysis of various potential channels through which immigrants impact on and are absorbed by the host economy.

\subsection{Theoretical framework}

We consider a model economy described by two sectors $(j=\{m, s\})$ that produce two different consumption products, manufacturing and services. Both sectors use one common and one specific factor. The common factor is competitively supplied and perfectly mobile between the two sectors. To get a better idea, we define the common

\footnotetext{
${ }^{16}$ The net replacement rate is commonly used as an indicator for both benefit generosity and incentives to work, see 2.A.1 for further details. There are, of course, many other indicators of flexibility (OECD, 2013). In the case of Denmark, indicators for labor market flexibility are unemployment duration and job finding procedures rather than wage adjustment; the replacement rate of 75 percent is the highest in our country sample.

${ }^{17}$ See 2.A.3 for the corresponding estimation results.
} 
Table 2.2: Evolution of employment in Europe by educational attainment and occupation

\begin{tabular}{|c|c|c|c|c|c|c|}
\hline \multirow[t]{2}{*}{ Country } & \multicolumn{2}{|c|}{ Lowest paid occupations } & \multicolumn{2}{|c|}{ Middling occupations } & \multicolumn{2}{|c|}{ Highest paid occupations } \\
\hline & $\begin{array}{l}\text { Shares } \\
\text { in } 1996 \\
(\text { in } \%)\end{array}$ & $\begin{array}{l}\text { Percentage } \\
\text { point change } \\
1996-2011\end{array}$ & $\begin{array}{l}\text { Shares } \\
\text { in } 1996 \\
(\text { in } \%)\end{array}$ & $\begin{array}{l}\text { Percentage } \\
\text { point change } \\
1996-2011\end{array}$ & $\begin{array}{l}\text { Shares } \\
\text { in } 1996 \\
(\text { in } \%)\end{array}$ & $\begin{array}{l}\text { Percentage } \\
\text { point change } \\
1996-2011\end{array}$ \\
\hline \multicolumn{7}{|c|}{ A: low-skilled workers } \\
\hline Austria & 33.84 & 13.29 & 53.01 & -11.08 & 12.86 & -2.21 \\
\hline Belgium & 30.93 & 11.73 & 53.37 & -8.45 & 14.02 & -2.34 \\
\hline Denmark & 49.77 & 4.53 & 39.80 & -9.25 & 8.53 & 6.20 \\
\hline France & 30.94 & 14.75 & 52.83 & -16.80 & 15.12 & 2.44 \\
\hline Germany & 33.00 & 14.20 & 48.23 & -9.16 & 15.57 & -4.07 \\
\hline Italy & 35.13 & 3.60 & 56.54 & -5.23 & 7.36 & 1.80 \\
\hline Netherlands & 29.55 & 15.56 & 45.88 & -7.32 & 17.87 & -3.24 \\
\hline Spain & 35.17 & 15.59 & 52.57 & -11.29 & 12.09 & -4.57 \\
\hline UK & 34.67 & 7.83 & 42.73 & -7.97 & 21.65 & 0.11 \\
\hline \multicolumn{7}{|c|}{ B: medium-skilled workers } \\
\hline Austria & 19.95 & 6.84 & 50.61 & -10.44 & 29.09 & 3.72 \\
\hline Belgium & 21.70 & 9.84 & 50.24 & -7.53 & 26.55 & -1.60 \\
\hline Denmark & 26.36 & 7.07 & 48.52 & -12.49 & 24.26 & 5.88 \\
\hline France & 19.19 & 12.64 & 52.47 & -16.44 & 26.36 & 4.32 \\
\hline Germany & 19.31 & 7.38 & 49.77 & -6.32 & 28.97 & -0.61 \\
\hline Italy & 17.28 & 7.36 & 42.98 & -5.12 & 38.72 & -2.60 \\
\hline Netherlands & 19.14 & 11.19 & 33.53 & -2.72 & 43.19 & -5.95 \\
\hline Spain & 27.32 & 15.84 & 43.18 & -6.36 & 28.19 & -9.02 \\
\hline UK & 19.68 & 15.27 & 50.18 & -17.57 & 28.88 & 3.01 \\
\hline \multicolumn{7}{|c|}{ C: high-skilled workers } \\
\hline Austria & 4.59 & 2.73 & 17.40 & -1.11 & 77.98 & -1.78 \\
\hline Belgium & 3.75 & 2.02 & 18.19 & -1.96 & 77.53 & 0.01 \\
\hline Denmark & 4.60 & 2.16 & 9.92 & -3.34 & 84.58 & 1.75 \\
\hline France & 2.50 & 5.80 & 11.46 & 0.19 & 85.30 & -6.05 \\
\hline Germany & 5.63 & 0.71 & 18.72 & -11.75 & 74.57 & 11.23 \\
\hline Italy & 3.82 & 2.70 & 8.77 & 3.87 & 87.07 & -6.88 \\
\hline Netherlands & 4.76 & 2.44 & 8.75 & -0.19 & 83.78 & -1.44 \\
\hline Spain & 9.22 & 4.10 & 23.32 & -1.48 & 66.82 & -2.44 \\
\hline UK & 5.49 & 7.19 & 12.93 & -1.60 & 80.70 & -5.20 \\
\hline
\end{tabular}

Source: Eurostat, own calculations.

Notes: Employment shares are based on total employment. The occupation classified according to 'ISCO 0' is omitted. Occupations classified according to 'ISCO 0' are omitted. Following Goos et al. (2011), we define the three occupation groups at the one-digit ISCO level as follows: Lowest paid occupations $=\operatorname{ISCO} 5 \& 9$; middling occupations $=\operatorname{ISCO} 4,6,7 \& 8$; highest paid occupations $=\operatorname{ISCO} 1,2 \& 3$.

factor as high-skilled labor and denote it by $H_{j} .{ }^{18}$ The sector-specific factors are two distinct labor inputs: medium-skilled workers $\left(l_{M}\right)$ employed in the manufacturing sector and low-skilled workers $\left(l_{L}\right)$ employed in the services sector. ${ }^{19}$

\footnotetext{
${ }^{18}$ To keep the analysis tractable we do not introduce for the moment physical capital explicitly in our analysis. However, under the assumption that physical capital moves freely between countries (at least in the case of advanced countries), including it in the analysis will cause a level effect due to the Le Chatelier-Samuelson principle (Felbermayr and Kohler, 2007; Neary, 1985). Therefore, the results of the analysis are not affected in qualitative terms.

${ }^{19}$ There exists a small but growing strand of literature addressing the importance of task content (e.g. interactive and communication intensive) in occupations in the context of immigration (cf. Peri and
} 
Goods are produced in the manufacturing sector by combining $H_{m}$ and the mediumskilled labor input $l_{M}$ using the following constant returns to scale (CRS) technology.

$$
Y_{m}=f_{m}\left(l_{M}, H_{m}\right)=\left[\alpha\left(l_{M}\right)^{\frac{\sigma_{m}-1}{\sigma_{m}}}+(1-\alpha)\left(H_{m}\right)^{\frac{\sigma_{m}-1}{\sigma_{m}}}\right]^{\frac{\sigma_{m}}{\sigma_{m}-1}} .
$$

Here, $\sigma_{m} \in[0,1]$ denotes the elasticity of substitution between the input factors, and $\alpha \in(0,1)$ is a distribution parameter. In the other sector, services are produced by combining $H_{s}$ and the low-skilled labor input $l_{L}$ using the technology

$$
Y_{s}=f_{s}\left(l_{L}, H_{s}\right)=\left[\beta\left(l_{L}\right)^{\frac{\sigma_{s}-1}{\sigma_{s}}}+(1-\beta)\left(H_{s}\right)^{\frac{\sigma_{s}-1}{\sigma_{s}}}\right]^{\frac{\sigma_{s}}{\sigma_{s}-1}}
$$

where $\sigma_{s} \in[0,1]$ denotes the elasticity of substitution in the service sector, and $\beta \in(0,1)$ is a distribution parameter.

To close the model, we define the domestic demand for both goods as follows. Total domestic product is defined by a CES technology

$$
X=\left(Y_{m}^{\frac{\sigma_{x}-1}{\sigma_{x}}}+Y_{s}^{\frac{\sigma_{x}-1}{\sigma_{x}}}\right)^{\frac{\sigma_{x}}{\sigma_{x}-1}}
$$

where the gross domestic product $X$ is maximized subject to the budget constraint while taking the manufacturing and service prices $\left(P_{m}, P_{s}\right)$ as given. Formally, this can be written as

$$
\max _{Y_{m}, Y_{s}}\left(Y_{m}^{\frac{\sigma_{x}-1}{\sigma_{x}}}+Y_{s}^{\frac{\sigma_{x}-1}{\sigma_{x}}}\right)^{\frac{\sigma_{x}}{\sigma_{x}-1}} \text {, s.t. } P_{m} Y_{m}+P_{s} Y_{s}=X,
$$

where $\sigma_{x} \in[0,1]$ denotes the elasticity of substitution in consumption. We take the price of $X$ as numeraire in the remaining part of the analysis. Consequently, all variables are defined in real terms and we assume no inflation. The solution to the optimization problem yields the following domestic demand functions.

$$
\begin{aligned}
Y_{m} & =X P_{m}^{-\sigma_{x}} \\
Y_{s} & =X P_{s}^{-\sigma_{x}}
\end{aligned}
$$

\subsubsection{Firm optimization problem}

After incurring a fixed cost, a representative firm chooses the optimal demand for inputs by minimizing its (variable) production cost, taking the factor prices as given. Formally,

Sparber, 2009). See also Autor and Dorn (2013), who define these input factors by a matching process of workers to occupations, e.g. 'routine' and 'manual' jobs, intensively used in each sector. Our framework could, in principle, be extended with respect to discrimination between immigrants and natives using a CES disaggregate in each skill group, where within skill heterogeneity could be defined by different sets of tasks that are performed by immigrants and natives and sorting into tasks is endogenous and based on comparative advantages. Therefore, incorporating this feature in the current framework would require a completely different type of modeling strategy, such as the "task-based" approach. However, we leave this feature to future research. 
the minimized cost function is

$$
C_{j}^{*}\left(w_{i}, w_{H}, Y_{j}\right)=\min _{l_{i}, H_{j}}\left\{w_{i} l_{i}+w_{H} H_{j}: f_{j}(\cdot)-Y_{j} \leq 0\right\}, \text { for } i=\{L, M\}, j=\{m, s\}
$$

and the corresponding minimized cost functions are

$$
\begin{aligned}
C_{m}^{*}\left(w_{M}, w_{H}, Y_{m}\right) & =\left[\alpha^{\sigma_{m}}\left(w_{M}\right)^{1-\sigma_{m}}+(1-\alpha)^{\sigma_{m}}\left(w_{H}\right)^{1-\sigma_{m}}\right]^{\frac{1}{1-\sigma_{m}}} Y_{m} \\
C_{s}^{*}\left(w_{L}, w_{H}, Y_{s}\right) & =\left[\beta^{\sigma_{s}}\left(w_{L}\right)^{1-\sigma_{s}}+(1-\beta)^{\sigma_{s}}\left(w_{H}\right)^{1-\sigma_{s}}\right]^{\frac{1}{1-\sigma_{s}}} Y_{s} .
\end{aligned}
$$

Now, by Shepards's Lemma, we obtain the optimal factor demands in each sector. In the manufacturing sector this implies

$$
\begin{aligned}
& \frac{\partial C_{m}^{*}(\cdot)}{\partial w_{M}}=\alpha^{\sigma_{m}}\left(\frac{c_{m}(\cdot)}{w_{M}}\right)^{\sigma_{m}} Y_{m}=l_{M} \\
& \frac{\partial C_{m}^{*}(\cdot)}{\partial w_{H}}=(1-\alpha)^{\sigma_{m}}\left(\frac{c_{m}(\cdot)}{w_{H}}\right)^{\sigma_{m}} Y_{m}=H_{m}
\end{aligned}
$$

and in the service sector

$$
\begin{aligned}
& \frac{\partial C_{s}^{*}(\cdot)}{\partial w_{L}}=\beta^{\sigma_{s}}\left(\frac{c_{s}(\cdot)}{w_{L}}\right)^{\sigma_{s}} Y_{s}=l_{L} \\
& \frac{\partial C_{s}^{*}(\cdot)}{\partial w_{H}}=(1-\beta)^{\sigma_{s}}\left(\frac{c_{s}(\cdot)}{w_{H}}\right)^{\sigma_{s}} Y_{s}=H_{s},
\end{aligned}
$$

where the unit costs are denoted by $c_{j}(\cdot)$, the term within the square brackets of Eqs. $(2.5)$.

In the next step, in each sector a representative firm maximizes the flow profit (i.e., net of fixed costs) with respect to goods price subject to the domestic demand function. ${ }^{20}$ Formally, this can be written as

$$
\max _{P_{j}}\left\{P_{j} Y_{j}-C_{j}^{*}\left(\cdot, Y_{j}\right)\right\}, \text { for } j=\{m, s\}
$$

subject respectively to (2.4.1), (2.4.2), (2.5.1), and (2.5.2). The solution yields the standard mark-up pricing behaviour

$$
\begin{aligned}
P_{m} & =\frac{1}{\rho} c_{m}\left(w_{M}, w_{H}\right) \\
P_{s} & =\frac{1}{\rho} c_{s}\left(w_{L}, w_{H}\right)
\end{aligned}
$$

\footnotetext{
${ }^{20}$ The representative firm in each sector is consistent with free entry if fixed costs are sufficiently high to make the entry of a second firm unprofitable, see Cahuc and Zylberberg (2004, Ch. 5.2) for a general discussion. Our assumption on monopolistic price setting in manufacturing sector is similar to Melitz type of trade models (cf. Gustafsson and Segerstrom, 2010). For monopolistic price setting in the context of trade and migration see Iranzo and Peri (2009).
} 
with $\rho=\frac{\sigma_{x}-1}{\sigma_{x}} \in[0,1]$. We now turn to the characteristics of the labor market.

\subsubsection{Wage setting and labor market frictions}

Taking into account the institutions of the European labor market and the empirical findings on low wage differentials between low- and medium-skilled workers in unionized firms (Dustmann and Schönberg, 2009), we assume union coverage in the low- and medium-skilled labor markets. That is, both types of workers are represented by two different labor unions who bargain their wages. There is, thus, an elastic institutional wage curve, reflecting the labor supply curve for the two skill groups as well as explaining the source of labor market frictions. In particular, the wage setting mechanism induces skill downgrading for some medium-skilled workers. Those workers who do not find a job in the manufacturing sector have the outside option to take a service job. For the low-skilled workers who do not find a job in the service sector, the outside option is unemployment.

It is worth pointing out that with collective bargaining, it is unclear why labor unions, e.g. representing medium-skilled type of jobs, should be in favor of discrimination between immigrants and natives with similar educational qualification. In fact, the possibility of discrimination is one of the main concerns of European labor unions regarding wage-dumping. Nevertheless, discriminating between immigrants and natives using the canonical approach and following the empirical evidence, one would expect an immigration-induced biased impact on the over-qualification rate of medium-skilled immigrants. Our results would, then, be a weighted average over natives' and immigrants' over-qualification rates and wages. This complicates the model too much and therefore we relegated this to future research. Downgrading of medium-skilled immigrants on arrival rather than after becoming not hired is captured implicitly by including the downgraded in the low-skilled endowment.

Following Booth (1995) and Layard et al. (2005), wages are determined by the right-tomanage bargaining solution, i.e., the negotiating parties only bargain over wages, whereas the optimal employment decisions are made by the firms.

At optimum, the firm's net gain is simply the flow of profits (П), i.e., net of fixed costs. The net gain for the labor union is simply the net result of the bargained wage and the outside option. Thus, the objective function of the labor unions in the manufacturing and service sectors, respectively, is given by

$$
\begin{aligned}
U_{m} & =\left(w_{M}-\bar{w}_{M}\right) l_{M} \\
U_{s} & =\left(w_{L}-\bar{w}_{L}\right) l_{L}
\end{aligned}
$$

where $\bar{w}_{i}, \forall i=\{L, M\}$ denotes the reference wage which is taken as given by each labor union.

The medium- and low-skilled wages are the result of the following maximization problem subject, respectively, to the labor demand equations, (2.6.1) and (3.35)

$$
\max _{w_{i}}\left\{\left(\left(w_{i}-\bar{w}_{i}\right) l_{i}\right)^{\delta_{j}} \Pi_{j}^{1-\delta_{j}}\right\}, \text { for } i=\{L, M\}, j=\{m, s\},
$$


where $\delta$ denotes the bargaining strength of the labor union. The solution of the wage bargaining yields the standard wage mark-up behavior ${ }^{21}$

$$
\begin{aligned}
w_{i} & =\left(1+\lambda_{i}\right) \bar{w}_{i}, \\
\lambda_{i} & =\frac{\delta_{j}}{\left(\sigma_{x}-1\right) \theta_{i}+\delta_{j}\left(1-\theta_{i}\right)\left(\sigma_{j}-1\right)}, \text { for } i=\{L, M\}, j=\{m, s\} .
\end{aligned}
$$

where $\theta_{i}=\frac{w_{i} l_{i}}{w_{i} l_{i}+w_{H} H_{j}}$ denotes the cost share of the labor input.

In keeping with the stylized facts, we assume that medium-skilled workers face the risk of holding a low skill job in the services sector when they cannot find employment in the manufacturing sector. We define the skill-downgrading rate of medium-skilled workers as

$$
d_{M}=1-\frac{l_{M}}{N_{M}}
$$

with $N_{M}$ the total medium-skilled labor force.

The reference wage of a medium-skilled worker $\left(\bar{w}_{M}\right)$ then is

$$
\bar{w}_{M}=\left(1-d_{M}\right) w_{M}+d_{M} w_{L} .
$$

Substituting this expression into (2.9) and rearranging, we obtain the wage curve of medium-skilled workers $\left(W C_{M}\right)$ in the manufacturing sector,

$$
w_{M}=\Phi\left(\lambda_{m}, d_{M}\right) w_{L},
$$

where $\Phi\left(\lambda_{m}, d_{M}\right)=\frac{\left(1+\lambda_{m}\right) d_{M}}{1-\left(1+\lambda_{m}\right)\left(1-d_{M}\right)}>1$ with the following properties: $\frac{\partial \Phi(\cdot)}{\partial \delta_{m}}>0$ and $\frac{\partial \Phi(\cdot)}{\partial d_{M}}<0$. Thus, a higher bargaining power and or level of employment yield stronger outside options for the union. In addition, any exogenous shock will induce a shift in (2.13) due to changes in $w_{L}$, and also induce a change in the shape of the wage curve due to changes in $d_{M}$.

In a similar way, the institutional wage curve for the low-skilled workers in the service sector can be derived. Since the outside option for these workers is unemployment, the reference wage of a low-skilled worker can be defined as

$$
\bar{w}_{L}=\left(1-u_{L}\right) w_{L}+u_{L} B_{L},
$$

where $B_{L}$ denotes the unemployment benefit and $u_{L}$ is the unemployment rate and is

\footnotetext{
${ }^{21}$ We refer the reader to $2 . \mathrm{C}$ for the derivation.
} 
defined by ${ }^{22}$

$$
u_{L}=1-\frac{l_{L}-d_{M} N_{M}}{N_{L}},
$$

where $N_{L}$ denotes the total low-skilled labor force.

Substituting (2.14) into the bargaining solution in the service sector (2.9) and rearranging yields the institutional wage curve $\left(W C_{L}\right)$ of low-skilled workers in the service sector:

$$
w_{L}=\Psi\left(\lambda_{s}, u_{L}\right) B_{L},
$$

where $\Psi\left(\lambda_{s}, u_{L}\right)=\frac{\left(1+\lambda_{s}\right) u_{L}}{1-\left(1+\lambda_{s}\right)\left(1-u_{L}\right)}>1$ with the following properties: $\frac{\partial \Psi(\cdot)}{\partial \delta_{s}}>0$ and $\frac{\partial \Psi(\cdot)}{\partial u_{L}}<$ 0 . Changes in the wage curve (2.16) and thus the labor market outcomes of the lowskilled workers depend on the characteristics of the unemployment benefits scheme. For example, under an exogenous unemployment benefits scheme, i.e., keeping the outside option of low-skilled workers fixed, exogenous shocks induce only changes in the elasticity of the wage curve (2.16), while under an endogenous benefit scheme, the wage curve will also shift as in (2.13). In the next section, we elaborate on the labor market implications for low and medium-skilled workers under these two different benefit schemes.

Finally, let the total endowment of the mobile factor be

$$
N_{H}=H_{m}+H_{s} \text {. }
$$

To sum up, the equilibrium of the model is characterized by fourteen endogenous variables $\left(X, Y_{m}, Y_{s}, P_{m}, P_{s}, d_{M}, u_{L}, l_{M}, l_{L}, H_{m}, H_{s}, w_{M}, w_{L}\right.$, and $\left.w_{H}\right)$ defined by Eqs. (2.1)(2.4.2), (3.35)-(2.8.2), (2.13), and (2.15)-(5.19).

\subsection{The general equilibrium solution}

In deriving the general equilibrium effects of exogenous changes in the labor supply due to immigration, we are particularly interested in the labor market reactions of low and medium-skilled workers under two different labor market regimes: exogenous vs. endogenous unemployment benefits. ${ }^{23}$

\footnotetext{
${ }^{22}$ The collective bargaining agreements are, in almost all EU countries, made at the industry level, see Venn (2009, Table 1) for an overview. Generally, a collective agreement between the labor union and the employers' association in one sector applies also to other firms in the same sector. However, it is less likely that cross-repercussions or the collective agreement in one sector would be recognized by other parties in other sectors. Thus, we assume that during the bargaining process, the labor union in the service sector does not take into account the crowding-out effect when negotiating over the low-skilled wage.

${ }^{23}$ Also note that when computing the general equilibrium effect, we take the cost shares as given at the initial steady state, which is consistent with assuming a Cobb-Douglas production technology.
} 


\subsubsection{Changes in the steady state}

We pursue the standard approach by computing the changes from the initial equilibrium by means of a log-linearization (Jones, 1965), i.e., $\hat{x}=\ln \left(\frac{x+\mathrm{d} x}{x}\right) \cong \frac{\mathrm{d} x}{x}$.

We commence as follows. Take the total differential of the log-difference of the labor demand functions (2.6.2) and (2.6.1), (2.7.2) and (3.35), to obtain

$$
\begin{gathered}
\hat{l}_{M}-\hat{H}_{m}=-\sigma_{m}\left(\hat{w}_{H}-\hat{w}_{M}\right), \\
\hat{l}_{L}-\hat{H}_{s}=-\sigma_{s}\left(\hat{w}_{H}-\hat{w}_{L}\right) .
\end{gathered}
$$

Taking the total domestic expenditure as given ${ }^{24}$, i.e., $\hat{X}=0$, then from the optimal demand conditions (2.4.1) and (2.4.2), log-linearization yields

$$
\begin{gathered}
\hat{Y}_{m}=-\sigma_{x} \hat{P}_{m}, \\
\hat{Y}_{s}=-\sigma_{x} \hat{P}_{s} .
\end{gathered}
$$

From the price-setting equations, (2.8.1) and (2.8.2), we obtain

$$
\begin{gathered}
\hat{P}_{m}=\theta_{M} \hat{w}_{M}+\left(1-\theta_{M}\right) \hat{w}_{H}, \\
\hat{P}_{s}=\theta_{L} \hat{w}_{L}+\left(1-\theta_{L}\right) \hat{w}_{H} .
\end{gathered}
$$

Next, let the share of low-skilled workers in the total employment of low skill service jobs be $s_{L}=\frac{\left(1-u_{L}\right) N_{L}}{l_{L}}$, and define the share of the mobile factor in the total employment working in the goods sector as $h=\frac{H_{m}}{N_{H}}$, then log-linearizing the labor market equilibrium conditions (2.11), (2.15), and the market clearing condition for the mobile factor (5.19), we obtain

$$
\begin{gathered}
\hat{l}_{M}=\left(\hat{N}_{M}-\bar{d}_{M} \hat{d}_{M}\right), \\
\hat{l}_{L}=s_{L}\left(\hat{N}_{L}-\bar{u}_{L} \hat{u}_{L}\right)+\left(1-s_{L}\right)\left(\hat{N}_{M}+\hat{d}_{M}\right), \\
\hat{N}_{H}=h \hat{H}_{m}+(1-h) \hat{H}_{s} .
\end{gathered}
$$

Thus, changes in low-skilled employment are weighted at the initial shares $s_{L}$.

Similarly, log-linearization of the wage curves (2.13) and (2.16) yields

$$
\begin{gathered}
\hat{w}_{M}=\varepsilon_{M} \hat{d}_{M}+\hat{w}_{L}, \\
\hat{w}_{L}=\varepsilon_{L} \hat{u}_{L}+\hat{B}_{L},
\end{gathered}
$$

where $\varepsilon_{M}=-\frac{\partial \Phi(\cdot)}{\partial d_{M}} \frac{d_{M}}{\Phi(\cdot)}$ and $\varepsilon_{L}=-\frac{\partial \Psi(\cdot)}{\partial u_{L}} \frac{u_{L}}{\Psi(\cdot)}$ denote the wage curve elasticities.

\footnotetext{
${ }^{24} \mathrm{~A}$ rationale for this assumption could be that it is the result of macroeconomic policies seeking to keep a balanced national account, see Felbermayr and Kohler (2007) for a similar argument.
} 
Finally, log-linearizing the production functions (2.1) and (2.2) yields

$$
\begin{gathered}
\hat{Y}_{m}=\theta_{M} \hat{l}_{M}+\left(1-\theta_{M}\right) \hat{H}_{m} \\
\hat{Y}_{s}=\theta_{L} \hat{l}_{L}+\left(1-\theta_{L}\right) \hat{H}_{s} .
\end{gathered}
$$

The general equilibrium effects are described by a system of thirteen equations, Eqs. (2.18.1)-(2.18.13), in the thirteen endogenous variables $\hat{Y}_{m}, \hat{Y}_{s}, \hat{P}_{m}, \hat{P}_{s}, \hat{H}_{m}, \hat{H}_{s}, \hat{l}_{M}, \hat{l}_{L}$, $\hat{d}_{M}, \hat{u}_{L}, \hat{w}_{H}, \hat{w}_{M}, \hat{w}_{L}$.

\subsubsection{Comparative statics with exogenous benefits}

Since we are interested in changes in the two friction variables, medium skill downgrading and low-skilled unemployment rates, the system derived above can be reduced and solved for $\hat{d}_{M}$ and $\hat{u}_{L}$. Moreover, to highlight the complementarity and substitution effects under the two different institutional settings, we focus first on the increase in medium-skilled endowments and its impact on the labor market friction variables. Thus, for the moment $\hat{N}_{L}=\hat{N}_{H}=0$. Also notice that the assumption of exogenous unemployment benefits implies $\hat{B}_{L}=0$. In the next section we relax this assumption.

We commence with the consumption goods market clearing condition. From (2.18.3), (2.18.5), and (2.18.12), we obtain

$$
\theta_{M} \hat{l}_{M}+\left(1-\theta_{M}\right) \hat{H}_{m}=-\sigma_{x}\left(\theta_{M} \hat{w}_{M}+\left(1-\theta_{M}\right) \hat{w}_{H}\right) .
$$

Similarly, combining (2.18.4), (2.18.6), and (2.18.13), we get

$$
\theta_{L} \hat{l}_{L}+\left(1-\theta_{L}\right) \hat{H}_{s}=-\sigma_{x}\left(\theta_{L} \hat{w}_{L}+\left(1-\theta_{L}\right) \hat{w}_{H}\right) .
$$

Next, we solve (2.18.1) and (2.18.2) w.r.t. the mobile factor and use the results in $\left(2.18 .3^{\prime}\right)$ and $\left(2.18 .4^{\prime}\right)$, respectively. After further rearrangement, we obtain

$$
\begin{gathered}
\hat{l}_{M}=\hat{w}_{H}\left(1-\theta_{M}\right)\left(\sigma_{m}-\sigma_{x}\right)-\hat{w}_{M} \Omega_{m}, \\
\hat{l}_{L}=\hat{w}_{H}\left(1-\theta_{L}\right)\left(\sigma_{s}-\sigma_{x}\right)-\hat{w}_{L} \Omega_{s} .
\end{gathered}
$$

Here $\Omega_{j}=\left(\theta_{i} \sigma_{j}+\left(1-\theta_{i}\right) \sigma_{x}\right)>0$ for $j=\{m, s\}, i=\{L, M\}$ is also interpreted in the literature as the generalized elasticity of substitution capturing both the elasticity in consumption and production (Jones, 1965).

Utilizing now the labor market conditions $(2.18 .7)$ and $(2.18 .8)$ in $\left(2.18 .3^{\prime \prime}\right)$ and $\left(2.18 .4^{\prime \prime}\right)$, respectively, yields

$$
\begin{aligned}
\left(\hat{N}_{M}-\bar{d}_{M} \hat{d}_{M}\right) & =\hat{w}_{H}\left(1-\theta_{M}\right)\left(\sigma_{m}-\sigma_{x}\right)-\hat{w}_{M} \Omega_{m} \\
-s_{L} \bar{u}_{L} \hat{u}_{L}+\left(1-s_{L}\right)\left(\hat{N}_{M}+\hat{d}_{M}\right) & =\hat{w}_{H}\left(1-\theta_{L}\right)\left(\sigma_{s}-\sigma_{x}\right)-\hat{w}_{L} \Omega_{s} .
\end{aligned}
$$

To eliminate the wage rates $\hat{w}_{M}$ and $\hat{w}_{L}$, we utilize $(2.18 .10)$ and $(2.18 .11)$ in $\left(2.18 .3^{\prime \prime \prime}\right)$ and $\left(2.18 .4^{\prime \prime \prime}\right)$. Then, we rearrange to obtain 


$$
\begin{aligned}
\hat{N}_{M}-\bar{d}_{M} \hat{d}_{M} & =\hat{w}_{H}\left(1-\theta_{M}\right)\left(\sigma_{m}-\sigma_{x}\right)-\left(\varepsilon_{M} \hat{d}_{M}+\varepsilon_{L} \hat{u}_{L}\right) \Omega_{m} \\
-s_{L} \bar{u}_{L} \hat{u}_{L}+\left(1-s_{L}\right)\left(\hat{N}_{M}+\hat{d}_{M}\right) & =\hat{w}_{H}\left(1-\theta_{L}\right)\left(\sigma_{s}-\sigma_{x}\right)-\varepsilon_{L} \hat{u}_{L} \Omega_{s} .
\end{aligned}
$$

Rearranging these two equations further, we derive two equations as a function of the variables of interest ${ }^{25}$

$$
\begin{aligned}
& \hat{d}_{M}=\frac{\hat{N}_{M}}{\mu_{M}}-\frac{\Omega_{m} \tilde{\varepsilon}_{L}}{\mu_{M}} \hat{u}_{L}-\frac{\left(1-\theta_{M}\right)\left(\sigma_{m}-\sigma_{x}\right)}{\mu_{M}} \hat{w}_{H} \\
& \hat{u}_{L}=\frac{\left(1-s_{L}\right)}{\mu_{L}}\left(\hat{N}_{M}+\hat{d}_{M}\right)-\frac{\left(1-\theta_{L}\right)\left(\sigma_{s}-\sigma_{x}\right)}{\mu_{L}} \hat{w}_{H},
\end{aligned}
$$

where $\mu_{M}=\left(\bar{d}_{M}+\Omega_{m} \tilde{\varepsilon}_{m}\right)>0$ and $\mu_{L}=\left(s_{L} \bar{u}_{L}+\Omega_{s} \tilde{\varepsilon}_{L}\right)>0$ with $\tilde{\varepsilon}_{i} \equiv-\varepsilon_{i}>0$ for $i=\{L, M\}$.

Now using (2.19.1) in (2.19.2) and performing some manipulations, we obtain

$$
\frac{\hat{u}_{L}}{\hat{N}_{M}}=\frac{\Gamma}{\Upsilon}-\frac{\Lambda}{\Upsilon} \frac{\hat{w}_{H}}{\hat{N}_{M}},
$$

where $\Gamma=\frac{\mu_{M} \mu_{L}+\Omega_{m} \tilde{\varepsilon}_{L}\left(1-s_{L}\right)}{\mu_{M} \mu_{L}}>0, \Upsilon=\frac{\left(1-s_{L}\right)\left(1+\mu_{M}\right)}{\mu_{M} \mu_{L}}>0$ and $\Lambda=$ $\frac{\left(1-\theta_{M}\right)\left(1-s_{L}\right)\left(\sigma_{m}-\sigma_{x}\right)+\left(1-\theta_{L}\right)\left(\sigma_{s}-\sigma_{x}\right) \mu_{L}}{\mu_{M} \mu_{L}} \lessgtr 0 .{ }^{26}$ The first term on the right-hand side denotes the first-order effect of an immigration-induced increase in the medium-skilled endowment. It is readily seen that this effect is positive, indicating a substitution effect of low-skilled workers due to the inflow medium-skilled workers.

The second term shows the feedback effect from factor price adjustments of the mobile factor. It is intuitively clear that the increase in medium-skilled endowments raises unambiguously the marginal productivity of the mobile factor, inducing an increase in $w_{H}$, i.e., $\frac{\hat{w}_{H}}{\hat{N}_{M}}>0$. However, the magnitude of the substitutability in consumption relative to that in production will determine the labor market outcomes of low- and mediumskilled workers.

Now substitute (2.20) back into (2.19.1) and rearrange to obtain

$$
\frac{\hat{d}_{M}}{\hat{N}_{M}}=\left(\frac{1}{\mu_{M}}-\frac{\eta \Gamma}{\Upsilon}\right)+\left(\frac{\eta \Lambda}{\Upsilon}-\chi\right) \frac{\hat{w}_{H}}{\hat{N}_{M}},
$$

where $\eta=\frac{\Omega_{m} \tilde{\varepsilon}_{M}}{\mu_{M}}$ and $\chi=\frac{\left(1-\theta_{M}\right)\left(\sigma_{m}-\sigma_{x}\right)}{\mu_{M}}$. Recalling the definitions of $\Gamma$ and $\Upsilon$ below

\footnotetext{
${ }^{25}$ It is important to notice that the equations in (2.19) contain a third endogenous variable, $\hat{w}_{H}$. Therefore, the system will be closed after utilizing the equilibrium labor market condition for the mobile factor, (2.18.9). However, changes in the factor price of the mobile factor are feedback effects and thus of second order. To keep the results readable, we focus on the direct effect and provide the intuition behind changes in $w_{H}$.

${ }^{26}$ The ambiguity of $\Lambda$ is the result of two forces: substitutability in consumption versus substitutability in production. To make this more obvious, consider the following special case where both sectors use Cobb-Douglas technology, i.e., $\sigma_{m}=\sigma_{s}=1$, while in consumption the assumption still holds that $\sigma_{x}>1$. It can then be easily shown that the numerator becomes unambiguously negative and simplifies to $-\left[\left(\sigma_{x}-1\right)\left(\left(1-\theta_{M}\right)\left(1-s_{L}\right)+\left(1-\theta_{L}\right) \mu_{L}\right)\right]$.
} 
(2.20), the right-hand side of (2.21) can be further manipulated to obtain

$$
\left(\frac{1}{\mu_{M}}-\frac{\eta \Gamma}{\Upsilon}\right)=\frac{s_{L} \bar{u}_{L}+\tilde{\varepsilon}_{L}\left(\Omega_{s}-\Omega_{m}\right)}{\mu_{M} \Upsilon} .
$$

This expression gives the direct effect of a supply shock of medium-skilled labor on the downgrading rate. The following proposition summarizes the main results.

Proposition 2.1 (Substitution effect under exogenous benefit). If the labor market of low- and medium-skilled workers has frictions, such that the medium-skilled workers' outside option is the low paid manual service job and low-skilled workers end up in unemployment, then under exogenous unemployment benefits, the immigration of medium-skilled workers will induce a substitution effect for the low-skilled workers. The reallocation of the mobile factor as well as the labor market outcomes for the mediumskilled workers depend crucially on the interaction between consumer preferences and the two production technologies. The stronger the substitutability is in the service sector $\left(\Omega_{s}>\Omega_{m}\right)$, the more the medium-skilled workers are downgraded into the service sector.

Intuitively, the mechanism of the adjustment process works as follows. The increase in the medium-skilled labor supply induces a higher downgrading threat and thus lowers the outside option of the medium-skilled labor union. The magnitude of the downward adjustment of wages for the medium-skilled depends on two forces: The elasticity of the medium-skilled wage curve as well as the substitutability in manufacturing production $\left(\sigma_{m}\right)$. An elastic wage curve together with low substitutability in the manufacturing sector imply an increase in the skill downgrading rate due to lower downward wage adjustment.

From Eq. (2.15) it is then immediately evident that the unemployment risk for the low-skilled workers rises. To counteract this effect, the low-skilled labor union agrees to downward wage adjustment. However, as in the manufacturing sector, wage adjustment depends on the substitutability between low-skilled workers and the mobile factor in the service sector $\left(\sigma_{s}\right)$ too. The interaction between these forces depends finally on consumer preferences, i.e., the substitutability between service and manufacturing goods captured by $\sigma_{x}$. Since both goods prices are endogenous, changes in the factor prices lead to changes in the goods prices, inducing shifts in the demands for the goods. More precisely, medium-skilled immigration increases the marginal productivity of high-skilled workers and with it their wages. Thus, the sector that more intensively utilizes the high-skilled labor input experiences a relative increase in the production cost and thus in the relative goods price. Consequently, that sector experiences an unfavorable goods demand effect.

To get a better intuition, recall the special case where $\sigma_{s}=\sigma_{m}=1$, i.e., Cobb-Douglas production technology, while the assumption regarding consumers preferences still holds, i.e., $\sigma_{x}>1$. This simplifies the first expression on the right-hand side in (2.21) to

$$
\frac{s_{L} \bar{u}_{L}+\tilde{\varepsilon}_{L}\left[\left(\sigma_{x} \theta_{L}+\left(1-\theta_{L}\right) \sigma_{s}\right)-\left(\sigma_{x} \theta_{M}+\left(1-\theta_{M}\right) \sigma_{m}\right)\right]}{\mu_{M} \Upsilon}=\frac{s_{L} \bar{u}_{L}+\tilde{\varepsilon}_{L}\left(\theta_{L}-\theta_{M}\right)\left(\sigma_{x}-1\right)}{\mu_{M} \Upsilon} .
$$

Now, consider the case $\theta_{M}>\theta_{L}$. This implies that the service sector uses the mobile factor more intensively. The inflow of medium-skilled immigrants increases the marginal productivity of high-skilled workers. Thus, the relative price of service goods increases, indicating a favorable shift in consumption for the manufacturing goods. This leads 
to a relative strong expansion of the manufacturing sector. This shift in consumption is also accompanied by a reallocation of the high-skilled workers (mobile factor) into the manufacturing sector. However, as discussed in the numerical part, for plausible parameter values $\frac{s_{L} \bar{u}_{L}}{\tilde{\varepsilon}_{L}\left(\sigma_{x}-1\right)}>\left(\theta_{M}-\theta_{L}\right)$, and hence $\mathrm{d} d_{M}>>0$. For all other cases, e.g. for sufficiently high $\sigma_{x}$ or $\tilde{\varepsilon}_{L}$, the first-order effect may become negative, i.e. $\mathrm{d} d_{M}<0$.

Summing up, the special case highlights the role of changes in the production cost structure under endogenous goods prices and when consumers prefer variety in consumption. Generally, the driving force is captured by the interaction between generalized elasticities indicating the substitutability in consumption and in production. ${ }^{27}$ Moreover, the immigration of medium-skilled workers displaces some of the low-skilled workers due to the relatively higher labor market rigidity denoted by the exogenous unemployment benefits.

\subsubsection{Comparative statics with endogenous benefits}

We now relax the assumption of a fixed benefit, and define instead the unemployment benefit as the proportion of the weighted average of the medium- and low-skilled wage rates. $^{28}$ In doing so, the outside option of low-skilled workers becomes endogenous and linked to the medium-skilled wage rate. Therefore, changes in the medium-skilled wage directly affect the low-skilled labor market. As we show below, this definition of endogeneity generates a source of labor market flexibility for low-skilled workers. ${ }^{29}$

Formally, we define the unemployment benefit as follows:

$$
B_{L}=\xi\left(\kappa w_{M}+(1-\kappa) w_{L}\right)
$$

where $\xi, \kappa \in(0,1)$ could be interpreted as the replacement rate and the weighting parameter, respectively. Then, log-linearizing $B_{L}$ yields

$$
\hat{B}_{L}=\phi_{M} \hat{w}_{M}+\left(1-\phi_{M}\right) \hat{w}_{L},
$$

where $0<\phi_{M}=\frac{\xi \kappa w_{M}}{B_{L}}<1$.

Substituting (2.22) into (2.18.11) and rearranging yields

$$
\hat{w}_{L}=\frac{\varepsilon_{L}}{\phi_{M}} \hat{u}_{L}+\hat{w}_{M} .
$$

Comparing $\left(2.18 .11^{\prime}\right)$ with $(2.18 .11)$, two differences become readily evident. First, the coefficient of $\hat{u}_{L}$ is now multiplied by $1 / \phi_{M}$. We interpret the parameter $\phi_{M}$ as the labor market policy parameter. That is, lowering $\phi_{M}$, e.g., due to a decline in the replacement rate $\xi$, induces an increase in the elasticity of the wage curve, making the low-skilled labor market more flexible. Indeed, as shown by the stylized facts, the group of countries

\footnotetext{
${ }^{27}$ Readers familiar with Autor and Dorn (2013) will recognize the main difference from our approach, as those authors assume no substitutability in the service sector.

${ }^{28}$ Weiss and Garloff (2009) show that in most European countries the level of benefits, unlike that in Anglo-Saxon countries, adjusts on a yearly basis and is closely tied to per capita income. Also Dustmann and Schönberg (2009) show for Germany that the wage differential between low- and medium-skilled workers in unionized firms is lower than for non-unionized firms.

${ }^{29}$ We provide in 2.C a formal discussion of the equilibrium properties of the two wage curves.
} 
with flexible labor markets denote on average about $7 \%$ percentage points a lower net replacement rate compared to the second group with rigid labor markets. The second difference is in the fact that now the low-skilled wage curve is affected by changes in the wages for the medium-skilled. More precisely, a decline in medium-skilled wages induces a downward shift in the low-skilled wage curve. This interdependency creates an additional source of flexibility.

Similar to the fixed benefit regime, we solve the system for the two friction variables in order to obtain a similar structure as in Eqs. (2.19). We commence by solving $\left(2.18 .3^{\prime \prime \prime}\right)$ and $\left(2.18 .4^{\prime \prime \prime}\right)$ for $\hat{w}_{M}$ and $\hat{w}_{L}$, respectively, to obtain

$$
\begin{aligned}
\hat{w}_{M} & =\frac{1}{\Omega_{m}}\left(\hat{w}_{H}\left(1-\theta_{M}\right)\left(\sigma_{m}-\sigma_{x}\right)-\left(\hat{N}_{M}-\bar{d}_{M} \hat{d}_{M}\right)\right) \\
\hat{w}_{L} & =\frac{1}{\Omega_{s}}\left(\hat{w}_{H}\left(1-\theta_{L}\right)\left(\sigma_{s}-\sigma_{x}\right)+\bar{u}_{L} s_{L} \hat{u}_{L}-\left(\hat{N}_{M}+\hat{d}_{M}\right)\right) .
\end{aligned}
$$

Subtracting the two equations yields

$$
\begin{aligned}
\hat{w}_{M}-\hat{w}_{L}= & \frac{1}{\Omega_{m}}\left(\hat{w}_{H}\left(1-\theta_{M}\right)\left(\sigma_{m}-\sigma_{x}\right)-\left(\hat{N}_{M}-\bar{d}_{M} \hat{d}_{M}\right)\right) \\
& -\frac{1}{\Omega_{s}}\left(\hat{w}_{H}\left(1-\theta_{L}\right)\left(\sigma_{s}-\sigma_{x}\right)+\bar{u}_{L} s_{L} \hat{u}_{L}-\left(\hat{N}_{M}+\hat{d}_{M}\right)\right) .
\end{aligned}
$$

Furthermore, from $(2.18 .10)$ and $\left(2.18 .11^{\prime}\right)$ we get

$$
\begin{aligned}
& \hat{w}_{M}-\hat{w}_{L}=-\tilde{\varepsilon}_{M} \hat{d}_{M} \\
& \hat{w}_{M}-\hat{w}_{L}=\frac{\tilde{\varepsilon}_{L}}{\phi_{M}} \hat{u}_{L} .
\end{aligned}
$$

Substituting $\left(2.18 .10^{\prime}\right)$ for $\left(\hat{w}_{M}-\hat{w}_{L}\right)$ in $\left(2.18 .11^{\prime \prime}\right)$ and $(2.23)$, and rearranging, yields the following system of two equations,

$$
\begin{aligned}
\hat{d}_{M}= & -\left(\tilde{\varepsilon}_{M}+\frac{\bar{d}_{M}}{\Omega_{m}}+\frac{\left(1-s_{L}\right)}{\Omega_{s}}\right)^{-1} \times \\
& {\left[\left(\frac{1-s_{L}}{\Omega_{s}}-\frac{1}{\Omega_{m}}\right) \hat{N}_{M}+\left(\frac{\left(1-\theta_{M}\right)\left(\sigma_{m}-\sigma_{x}\right)}{\Omega_{m}}-\frac{\left(1-\theta_{L}\right)\left(\sigma_{s}-\sigma_{x}\right)}{\Omega_{s}}\right) \hat{w}_{H}-\frac{\bar{u}_{L} s_{L}}{\Omega_{s}} \hat{u}_{L}\right] } \\
\hat{u}_{L}= & -\frac{\tilde{\varepsilon}_{M}}{\tilde{\varepsilon}_{L}} \phi_{M} \hat{d}_{M} .
\end{aligned}
$$

Before proceeding, it might helpful to consider first the behavior of Eq. (2.25). The effect of an increase in skill downgrading on the low-skilled unemployment rate is mitigated by lowering $\phi_{M}$. As already mentioned above, this is the result of a stronger responsiveness of the low-skilled wage curve to changes in the unemployment rate under endogenous unemployment benefits. However, to drive the full effect, we need to solve (2.24) and (2.25) for the two variables $\hat{d}_{M}$ and $\hat{u}_{L}$. In doing so, define for convenience the following expressions: $v=\left(\tilde{\varepsilon}_{M}+\frac{\bar{d}_{M}}{\Omega_{m}}+\frac{\left(1-s_{L}\right)}{\Omega_{s}}\right)>0, \Delta=\left(\frac{1-s_{L}}{\Omega_{s}}-\frac{1}{\Omega_{m}}\right) \lesseqgtr 0$, and $\Sigma=\left(\frac{\left(1-\theta_{M}\right)\left(\sigma_{m}-\sigma_{x}\right)}{\Omega_{m}}-\frac{\left(1-\theta_{L}\right)\left(\sigma_{s}-\sigma_{x}\right)}{\Omega_{s}}\right)=\left(\frac{\sigma_{m}}{\Omega_{m}}-\frac{\sigma_{s}}{\Omega_{s}}\right) \lesseqgtr 0$.

Then, we combine (2.24) and (2.25), divide by $\hat{N}_{M}$, and rearrange to obtain 


$$
\begin{aligned}
\frac{\hat{u}_{L}}{\hat{N}_{M}} & =\frac{\tilde{\varepsilon}_{M} \phi_{M}}{\tilde{\varepsilon}_{L} v}\left[\Delta+\Sigma \frac{\hat{w}_{H}}{\hat{N}_{M}}-\frac{\bar{u}_{L} s_{L}}{\Omega_{s}} \frac{\hat{u}_{L}}{\hat{N}_{M}}\right] \\
\Rightarrow \frac{\hat{u}_{L}}{\hat{N}_{M}} & =\left(\frac{\tilde{\varepsilon}_{M} \phi_{M} \Omega_{s}}{\tilde{\varepsilon}_{M} \phi_{M} \bar{u}_{L} s_{L}+\tilde{\varepsilon}_{L} v \Omega_{s}}\right) \Delta+\left(\frac{\tilde{\varepsilon}_{M} \phi_{M} \Omega_{s} \Sigma}{\tilde{\varepsilon}_{M} \phi_{M} \bar{u}_{L} s_{L}+\tilde{\varepsilon}_{L} v \Omega_{s}}\right) \frac{\hat{w}_{H}}{\hat{N}_{M}} .
\end{aligned}
$$

Dividing (2.25) by $\hat{N}_{M}$ and utilizing (2.26) yields

$$
\frac{\hat{d}_{M}}{\hat{N}_{M}}=-\left(\frac{\tilde{\varepsilon}_{L} \Omega_{s}}{\tilde{\varepsilon}_{M} \phi_{M} \bar{u}_{L} s_{L}+\tilde{\varepsilon}_{L} v \Omega_{s}}\right) \Delta-\left(\frac{\tilde{\varepsilon}_{L} \Omega_{s} \Sigma}{\tilde{\varepsilon}_{M} \phi_{M} \bar{u}_{L} s_{L}+\tilde{\varepsilon}_{L} v \Omega_{s}}\right) \frac{\hat{w}_{H}}{\hat{N}_{M}} .
$$

Equations (2.26) and (2.27) are the analogs to (2.20) and (2.21) derived previously under the exogenous benefits regime. Comparing the first terms on the right-hand sides in (2.26) and in (2.20), one observes that in Eq. (2.20), under exogenous benefits, the sign of the first term is always positive. However, the direct effect in the case of endogenous benefits is ambiguous and is determined by the term $\Delta$. To verify the sign of the first term in (2.26), recall the definition of $\Delta$, and utilize $\Omega_{m}$ and $\Omega_{s}$ defined earlier in Eqs. $\left(2.18 .3^{\prime \prime}\right)$ and $\left(2.18 .4^{\prime \prime}\right)$. Then, it follows ${ }^{30}$

$$
\Delta=\left(\frac{1-s_{L}}{\Omega_{s}}-\frac{1}{\Omega_{m}}\right)=-\frac{s_{L}}{\Omega_{s}}-\frac{\Omega_{s}-\Omega_{m}}{\Omega_{m} \Omega_{s}},
$$

which determines the sign of the direct effect of medium-skilled immigration on the skill downgrading rate. The next proposition summarizes the main results.

Proposition 2.2 (Complementarity effect under endogenous benefit). If low- and medium-skilled labor markets possess frictions where labor supply is defined by an institutional wage setting curve, then for a stronger substitutability in the service sector, i.e., $\Omega_{s}>\Omega_{m}$, an immigration-induced increase in the medium-skilled endowment raises the incidence of skill downgrading followed by a reduction in low-skilled unemployment, implying a complementarity effect. In addition, the more flexible is the low-skilled labor market (lower $\phi_{M}$ ), the less severe is the adverse effect of higher incidence of skill downgrading.

The intuition behind the driving force is similar to that of Proposition 2.1, except that now due to the endogenous unemployment benefits, the low-skilled labor market has become more flexible as indicated in Eq. (2.18.11'). Put differently, a higher risk of unemployment due to the migration-induced increase in skill downgrading implies a stronger downward wage adjustment for the low-skilled workers. Therefore, a relatively stronger substitutability in the service sector $\left(\Omega_{s}>\Omega_{m}\right)$ induces an increase in the demand for the low-skilled labor input in the service sector. This contrasts with the exogenous benefit scheme where the adjustment process was rather rigid. It, therefore, highlights the importance of labor market institutions.

Thus, we are able to provide a potential rationale behind the role of institutions in

\footnotetext{
${ }^{30}$ To see the sign more clearly, it is worthwhile to look again at the special case where $\sigma_{m}=\sigma_{s}=1$, while $\sigma_{x}>1$. We then obtain $\Delta=\frac{-s_{L}}{\Omega_{s}}-\frac{\left(\sigma_{x}-1\right)\left(\theta_{m}-\theta_{s}\right)}{\Omega_{s} \Omega_{m}}<0$.
} 
determining the relationship between the low-skilled unemployment rate and the skill downgrading rate across the European countries, shown in Figure 2.1. That is, lowskilled workers in countries, like France, that have a rather rigid labor market (high $\left.\phi_{M}\right)$ are more vulnerable in terms of employment outcomes to exogenous shocks like immigration. Formally, this can be shown as follows. Consider two identical countries, $F$ and $D$, differing only in the policy parameter $\phi_{M}$. Comparing the direct effect of mediumskilled immigration on the low-skilled unemployment rate, i.e., keeping the second term constant, yields

$$
\begin{aligned}
\left.\frac{\hat{u}_{L}^{F}}{\hat{N}_{M}}\right|_{\hat{w}_{H}=0} & \left.\lesseqgtr \frac{\hat{u}_{L}^{D}}{\hat{N}_{M}}\right|_{\frac{\hat{w}_{H}}{\tilde{N}_{M}}=0} \\
\left(\frac{\tilde{\varepsilon}_{M} \phi_{M}^{F} \Omega_{s}}{\tilde{\varepsilon}_{M} \phi_{M}^{F} \bar{u}_{L} s_{L}+\tilde{\varepsilon}_{L} v \Omega_{s}}\right) \Delta & \lesseqgtr\left(\frac{\tilde{\varepsilon}_{M} \phi_{M}^{D} \Omega_{s}}{\tilde{\varepsilon}_{M} \phi_{M}^{D} \bar{u}_{L} s_{L}+\tilde{\varepsilon}_{L} v \Omega_{s}}\right) \Delta \\
\phi_{M}^{F} & \lesseqgtr \phi_{M}^{D} .
\end{aligned}
$$

In conclusion, the comparative static analysis emphasizes two important findings. First, following the medium-skilled immigration, the incidence of skill downgrading of mediumskilled workers together with a endogenous benefits scheme induces an increase in lowskilled employment in the service sector. This is in line with the stylized facts discussed earlier, about the pattern of immigration and employment composition in many advanced countries. Second, the production technology in the service sector is a crucial determinant explaining the labor market outcomes. This differs from the discussion of Autor and Dorn (2013), who emphasize only the roles of consumer preferences and of production technology in the goods sector. Moreover, it is worth pointing out that in the special case of a Cobb-Douglas production technology, the results are driven by the factor cost share, i.e. the factor intensity. In the next section we provide a quantitative assessment and examine the general equilibrium effects of different immigration scenarios.

\subsection{Numerical assessment}

In this section, we provide a numerical analysis of long-run effects of various migration scenarios on the labor market and the economy, accounting for flexible and rigid labor market institutions. Based on our empirical evidence presented in Figure 2.1 (and Table 2.5 in 2.A.2), we consider Germany as the representative of the group of countries (Austria, Belgium, Finland, Germany) with flexible labor markets, characterized by low net replacement rates and a negative relationship between low-skilled unemployment and medium-skilled over-qualification rates, while France is considered to represent the second group of countries (Denmark, Ireland, Greece, France, Italy, the Netherlands, Sweden) with inflexible institutional settings, characterized by high net replacement rates and a positive correlation between the unemployment and downgrading rates. We use the EUKLEMS database to calibrate the parameter values for Germany and France. Furthermore, we take 2004 as the benchmark year. We then use the calibrated parameters and benchmark values of the variables to simulate the impact of migration on output and the labor market variables. In doing so, we modify the model by including physical and 
human capital input factors in the production function. Here we consider the special case of the production function, i.e. a Cobb-Douglas technology, while the assumption on consumption preferences $\left(\sigma_{x}>1\right)$ holds. Note that, as discussed above, in this case the results depend on the the cost shares (i.e., factor intensity) in both sectors. In 2.B, we outline the model as well as the values of the benchmark parameters and variables.

\subsubsection{Migration scenarios}

Similarly to Felbermayr and Kohler (2007), we simulate migration scenarios for different skill compositions of migration flows. Table 2.3 gives an overview of our simulation scenarios. In scenario (I), we assume a proportional increase in all skill levels, which

Table 2.3: Simulation scenarios

\begin{tabular}{cl}
\hline \hline Scenario & Description \\
\hline (I) & Perfectly balanced immigration \\
(II) & Inflow at tails \\
(III) & Medium- and high-skilled inflow \\
(IV) & High-skilled inflow only \\
(V) & Medium-skilled inflow only \\
(VI) & Low-skilled inflow only \\
\hline
\end{tabular}

approximately resembles the Dutch immigration scenario, see Muysken and Ziesemer (2011). In scenario (II), we assume the immigration to be composed of $75 \%$ low-skilled and $25 \%$ high-skilled labor. As pointed out by Felbermayr and Kohler (2007), this denotes the most realistic case for the past in the OECD countries, as it models a bimodality in migration flows with a bias towards low-skilled migration. We also simulate the model for the current migration pattern within the EU (scenario (III)), where the majority of migrants from the new member states (Poland and the Baltic states) are predominantly young with medium or high levels of skill (Blanchflower et al., 2007).

In doing so, in scenario (III), we use as a benchmark the relative share of high-skilled foreign labor force in the U.S., which can be seen as a target value, and subtract from that the values for Germany and France. ${ }^{31}$ We, then, compute the percentage inflows so that the overall size of the inflows equals $10 \%$ of the total labor force. The resulting inflow consists in Germany of $55.7 \%$ and $44.3 \%$ and in France of $45.30 \%$ and $54.70 \%$ of medium- and high-skilled immigrants, respectively. ${ }^{32}$

\footnotetext{
${ }^{31}$ As used in the migration literature, see Ziesemer (2011), we take the World Bank data on migration stocks, which provides information on the educational attainments of immigrants and the total labor force. However, the Migration Data provides only information from 1975 to 2000, which we use as a proxy.

${ }^{32}$ The computation is as follows: the share of high-skilled immigrants in the U.S. labor force is about $10.91 \%$ and in the German (France) labor force about $6.48 \%$ (5.44\%) in 2000, which makes a difference of $4.43 \%(5.47 \%)$ in case of Germany (France). Therefore, in scenario (III), the high-skilled labor force has to rise by $44.30 \%=4.43 \% \times\left(N / N_{H}\right)$ which gives us a total increase in the medium-skilled labor force by $8.80 \%=(10 \%-4.43 \%) \times\left(N / N_{M}\right)$ in Germany.
} 
We also assess the quantitative impact of each skill group separately in the scenarios (IV)-(VI). Furthermore, to ensure comparability between the different cases and due to the fact that just under $10 \%$ of the German workforce are foreign born, all scenarios are specified in such a way that the overall size of the inflow is approximately $10 \%$ of the initial labor force. Finally, we assume full adjustment of the capital stock. Hence, the results indicate long-run effects. It is also important to notice that the service sector is slightly more high skill intensive than the manufacturing sector.

\subsubsection{Simulation results}

We organize the discussion of our findings around the following core numerical results.

Numerical Result 1: A perfectly balanced inflow of all three skill groups is consistent with no change in relative wages, unemployment rates, and skill downgrading.

This is a standard, intuitively plausible result, since a proportional increase of the labor force implies a scale effect due to the full capital adjustments, confirming earlier empirical results (Dustmann et al., 2008). However, notice that differences in production functions can cause small differences in the pure scale effect of $10 \%$.

Numerical Result 2: The welfare effects of unbalanced recent migration patterns dominate substantially that of past patterns.

The unbalanced migration patterns are represented by scenario (II) and (III). The past immigration patterns, dominated by low-skilled migration, has lead to a modest increase of GDP per capita (1 to 1.4 percent), while the recent immigration pattern, dominated by high-skilled immigrants, raises GDP per capita by a magnitude of 2.4 to 4.4 percent. Hence, our numerical exercise highlights the quantitative importance of the skill structure of immigrants.

In addition, scenario (II) illustrates nicely that due to the relative scarcity of medium skilled workers following this migration pattern, medium-skilled wages increase relatively to high-skilled wages and low-skilled wages, and the over-qualification rate decreases. Moreover, the price in manufacturing increases relative to that of services, because lowskilled immigration increases relative supply of services. This is consistent with our model above.

Numerical Result 3: Institutional settings play an important role both in terms of magnitude and outcomes for low- and medium-skilled workers. Moreover, mediumskilled immigration causes a wage polarization effect.

First, observe the role of institutional settings in the unbalanced recent immigration scenario (III), with substantial share of high-skilled immigrants, and the pure highskilled immigration scenario (IV). The unemployment rate of low-skilled workers decreases and the skill downgrading rate increases in both endogenous (Germany) and exogenous (France) benefit regimes. The rationale behind this similar effect is twofold. In case of France, with rigid benefits, the complementarity effect is purely demand-driven, where due to skill downgrading and the relative high intensity of high-skilled workers in the service sector the influx of high-skilled immigrants causes the relative price of service goods to decline. This favorable supply and demand shift towards service goods induces 
an expansionary effect for the service sector, and hence increasing the demand for lowskilled service jobs. These observations are consistent with our model and the empirical findings on scale and intensity effects driven by immigration (González and Ortega, 2011). They also confirm our stylized facts about the recent increase in the supply of mediumand high-skilled immigrants in Table 2.1 and low-skilled intensive jobs in Table 2.2 in many advanced countries.

In the case of Germany, reflecting a flexible labor market, the complementarity effect is induced by a mixture of goods demand effect, i.e. a relative decline in service goods prices, and of endogenous benefit effect, i.e. a decline in the reservation wage of low-skilled workers. The pure complementarity and substitution effects discussed by Proposition 2.1 and 2.2 are reconciled by immigration scenario (V), where all migration consists of medium-skilled persons. Here, due to the nature of wage setting the influx of medium-skilled workers causes the over-qualification rate to increase. The importance of institutions is readily seen. While in France, low-skilled workers are considerably displaced due the inflow of medium-skilled workers, in Germany, the unemployment rate of low-skilled workers is decreased.

Second, scenario (V) shows the so-called polarization effect, where the medium-skilled wage declines relative to both high-skilled and low-skilled wages. As a consequence of this strong wage decline, employment of medium-skilled workers in the manufacturing sector has risen considerably, while that sector expanded even stronger than the services sector and prices decreased in manufacturing relative to services - the latter two are unique events.

A third interesting observation derived from scenario $(\mathrm{V})$ is that this is the only scenario in which output per capita declines as a consequence of migration. The reason lies in the low capital accumulation in the services sector compared to the other scenarios, induced by following four effects: (i) human capital is reallocated from services, where it has a higher elasticity of production, to manufactures, because of the relative expansion of manufactures; (ii) substitution of cheap low skilled labor for capital in comparison with scenario (III); (iii) unemployment is reduced less than in the realistic scenario (III), leading to relatively less additional low-skilled employment in services; and (iv) the decreased overall demand together with the strong decline in medium-skilled wages.

Finally, scenarios (IV) and (VI) illustrate the impact of pure high-skilled and pure lowskilled migration, respectively. These scenarios follow the patterns we have already discussed above. Table 2.4 reports the results of the numerical exercise. 


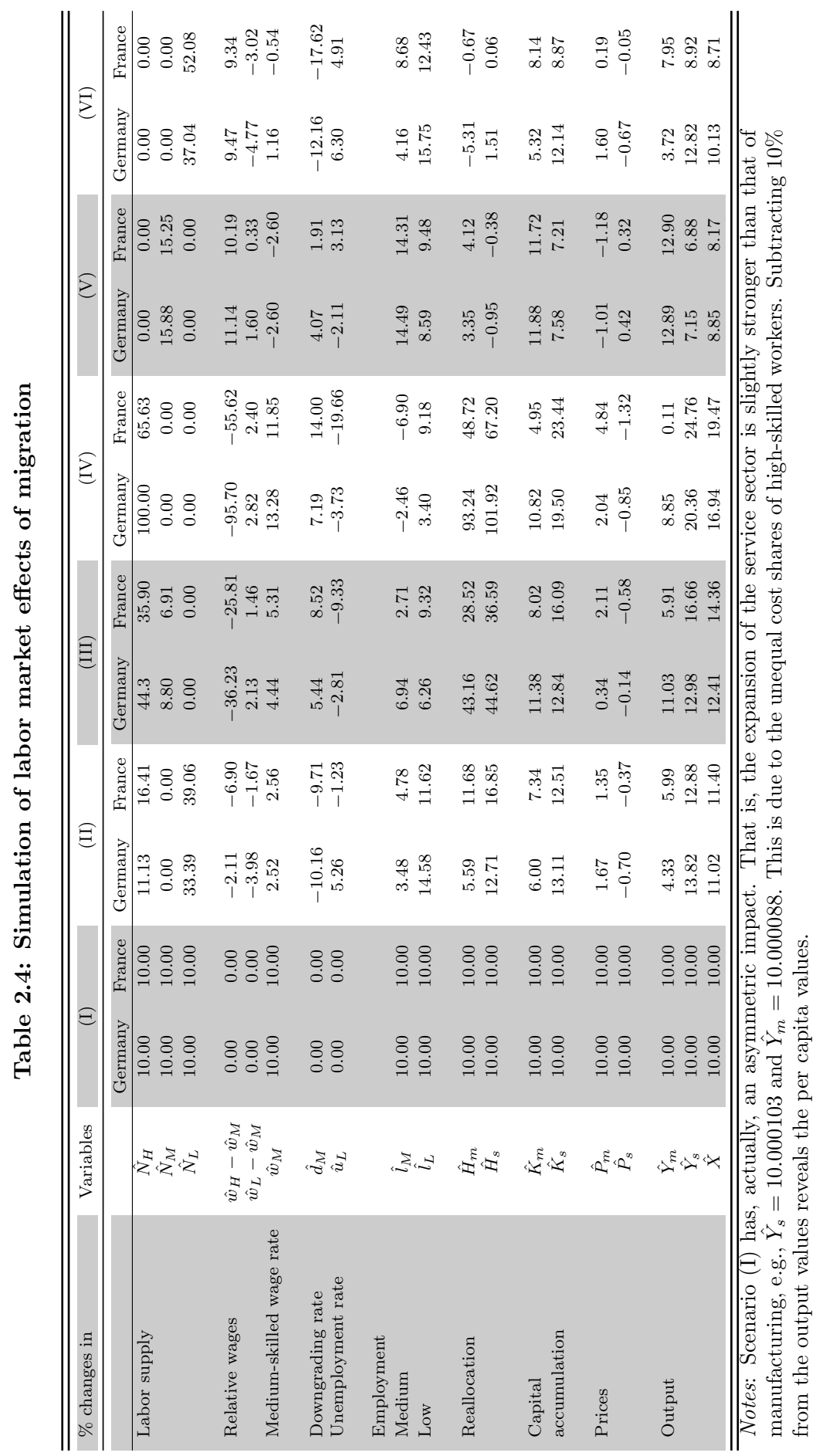




\subsection{Conclusion}

In this chapter we present a theoretical model, motivated by our stylized facts, with two major sectors (manufacturing and services) and heterogeneous labor markets (high, medium-, and low- skilled) to analyze the impact of various skill compositions of immigration. While the impact of migration flows with different skills on the sectoral decomposition, goods prices, and labor market outcomes for the receiving country has been analyzed separately, to our knowledge, no paper exists that examines the impact of immigration in a general equilibrium framework combining low-skilled unemployment and medium-skilled downgrading incidence under two different institutional settings. At the same time, the existing literature has paid less attention to the repercussions of medium-skilled immigration considering simultaneously its implication for low-skilled workers. Our stylized facts highlight the importance of labor market institutions and the analytical solution of the model verifies that it is able to reproduce these stylized facts. We have also quantified the impact of different migration scenarios on the labor market and GDP. The following outcomes are at the core of our analysis.

First, we elaborate on the impact of institutions on the labor market performance of low-skilled workers, who are most vulnerable to economic shocks. In particular, our comparative static analysis reveals that in flexible labor markets, captured by an endogenous benefit scheme, medium-skilled immigration generates a complementarity effect, which enhances low-skilled employment, while in more rigid labor markets, it induces a substitution effect, increasing low-skilled unemployment. The rationale behind these different outcomes is that the outside option of unemployed low-skilled workers is more responsive to economic shocks under the flexible regime, mitigating adverse employment effects.

Based on our empirical evidence, we consider France and Germany as the representatives of the two groups of countries with different labor market institutions and quantify the impact of different immigration scenarios. In line with the usual conclusion regarding the effect of skilled and unskilled immigration, we also find that the immigration of the high-skilled is beneficial to the receiving economy (increasing GDP per capita between 6\% and 10\%). Focusing on medium-skilled immigration, we find new insights that augment those in the current literature. The results reveal, indeed, that immigration of mediumskilled workers can generate partly favorable economic outcomes. Under the flexible labor market regime, medium-skilled immigration improves the labor market conditions for lowskilled workers, while under the rigid regime it induces a substitution effect, displacing low-skilled workers. Moreover, even without accounting for dynamic labor market effects, such as the assimilation process, or the aging effect, medium-skilled immigration can be beneficial for the host economy. For instance, our simulation analysis of the impact on GDP per capita of the past (Scenario II) and the recent (Scenario III) migration pattern shows that in the latter case the welfare effects are considerably higher, a difference of 1.4 to $3 \%$.

Furthermore, the endogenous goods prices resulting from price-setting behavior are an important economic mechanism in explaining the substitutability between different types of labor. Our findings reveal that labor migration, irrespective of the skill structure, may also generate a productivity effect for firms by lowering production costs. This in 
turn explains changes in the skill intensity across sectors. Moreover, the importance of institutional characteristics as well as the welfare and labor market implications of medium-skilled immigration give an important insight for designing migration and labor market policies to satisfy the future labor replacement demand, for instance, due to aging. 


\section{Appendix}

\section{A Empirical analysis}

\section{A.1 Data}

For the underlying empirical analysis, we have drawn data from two sources. Information on unemployment rate and over-qualification are based on the annual labor force survey data and are drawn from the Eurostat. We follow the commonly used approach in the literature and compute the medium-skilled over-qualification rate according to ISCO-ISCED mismatch. More precisely, the share of medium-skilled workers (ISCED 3 and 4), who are employed in occupations according to ISCO 5 and 9, are defined as over-qualified. Annual data on GDP are taken from OECD Statistics. Finally, we collect data on the net replacement rate, which includes social assistance and housing benefits, from the OECD Tax and Benefits statistics, provided in the excel file "GRR EN", variable "NRR incl SA HB (AW)".33

\section{A.2 Estimation of the correlation between over-qualification and unemployment rates}

We estimate the relation between the low-skilled unemployment rate, $u_{L}$, and the log of mediumskilled downgrading rate, $\log \left(d_{M}\right)$, using the lagged dependent variable, $u_{L}(t-1)$. We control for the financial crisis and business cycles by using the lagged growth rate of GDP per capita, $g_{y}(t-1)$, because employment tends to follow economic growth with some lag. We control for technical change by using a time trend, $t$. We assume heterogeneous coefficients throughout. In order to avoid serial correlation bias, the first-order autocorrelation, $\operatorname{AR}(1)$, is taken into account. The system of equations can then be written as:

$$
\begin{aligned}
& u_{i}(t)=\text { const }_{i}+\gamma_{i} u_{i}(t-1)+\beta_{1, i} \log \left(d_{M}(t)\right)+\beta_{2, i} g_{y}(t-1)+\beta_{3, i} t+v_{i}(t), \\
& v_{i}(t)=\rho v_{i}(t-1)+\epsilon_{i}(t),
\end{aligned}
$$

where $v_{i, t}$ is the residual, const ${ }_{i}$ denotes the country-specific constant, and $i$ is the country index. ${ }^{34}$

In case of endogeneity we would use the lags of the regressors as instruments. A Durbin-Wu-Hausman (DWH) test suggests that over-qualification is not endogenous, see Wooldridge (2002, Chap. 6). As the other regressors have lags anyway, we do not use instrumental variable (IV) methods. Since unemployment rates are likely to be dependent on those of the other countries we expect to have contemporaneous correlation of the residuals and therefore we use the SUR (seemingly unrelated regression) method. The results are summarized in the Table 2.5 with $p$-values in parentheses.

Over-qualification has a significantly negative sign for Belgium, Germany, Finland and Austria. We find significantly positive signs for Denmark, Ireland, Greece, France, Italy, the Netherlands and Sweden. The coefficients of the lagged dependent variable are positive and significant with the exception of Belgium and Sweden. But they are all below unity, indicating the absence of unit roots in the presence of control variables. ${ }^{35}$ The GDP growth variable is always negative, but insignificant for Austria, Portugal

\footnotetext{
${ }^{33}$ Due to availability of cross-country harmonized data on institutional settings, the net replacement ratio is used as a proxy.

${ }^{34}$ Since the replacement rate is rather time invariant over the sample period, it is not possible to use it as a separate variable in the estimation, but it is subsumed under the country specific constant.

${ }^{35}$ Performing standard unit root tests for the over-qualification variable provides mixed evidence for and against unit roots. However, for our small sample, unit root tests have pertinently low power (Baltagi, 2008, chapter 12), meaning that too often unit roots are suggested. Therefore we prefer to lean against the unit root hypothesis. For the unemployment rate, lagged variables for all countries have coefficients below unity, actually between 0.05 and 0.9 ; in case of unit roots at least some should be at or above unity and therefore we have at best a near-unit root here. GDP variables are well-known to
} 
Table 2.5: Correlation between medium-skilled over-qualification and low-skilled unemployment rates

\begin{tabular}{|c|c|c|c|c|c|c|c|c|c|}
\hline Country & const & $u_{L}(t-1)$ & $\log \left(d_{M}\right)$ & $g_{y}(t-1)$ & $t$ & $\operatorname{AR}(1)$ & Adjusted $R^{2}$ & DWH-Test & Obs. \\
\hline Belgium & $\begin{array}{l}-0.1660 \\
(0.6434)\end{array}$ & $\begin{array}{c}0.1015 \\
(0.4439)\end{array}$ & $\begin{array}{l}-0.1022 \\
(0.0002)\end{array}$ & $\begin{array}{l}-0.1453 \\
(0.0721)\end{array}$ & $\begin{array}{l}0.0083 \\
(0.478)\end{array}$ & $\begin{array}{c}0.915 \\
(0.000)\end{array}$ & 0.5966 & 1.7021 & 19 \\
\hline Denmark & $\begin{array}{c}0.0747 \\
(0.0078)\end{array}$ & $\begin{array}{l}0.7870 \\
(0.000)\end{array}$ & $\begin{array}{c}0.0368 \\
(0.0484)\end{array}$ & $\begin{array}{c}-0.4648 \\
(0.000)\end{array}$ & $\begin{array}{l}-0.0005 \\
(0.1264)\end{array}$ & $\begin{array}{l}-0.3881 \\
(0.0003)\end{array}$ & 0.7537 & 2.3047 & 19 \\
\hline Germany & $\begin{array}{l}-0.1822 \\
(0.0706)\end{array}$ & $\begin{array}{l}0.8087 \\
(0.000)\end{array}$ & $\begin{array}{c}-0.1279 \\
(0.034)\end{array}$ & $\begin{array}{l}-0.0500 \\
(0.6374)\end{array}$ & $\begin{array}{c}0.0014 \\
(0.2205)\end{array}$ & $\begin{array}{c}0.2713 \\
(0.1498)\end{array}$ & 0.7256 & 1.4126 & 16 \\
\hline Ireland & $\begin{array}{c}0.2043 \\
(0.0069)\end{array}$ & $\begin{array}{c}0.7059 \\
(0.000)\end{array}$ & $\begin{array}{c}0.1031 \\
(0.0288)\end{array}$ & $\begin{array}{c}-0.4766 \\
(0.000)\end{array}$ & $\begin{array}{l}-0.0017 \\
(0.0672)\end{array}$ & $\begin{array}{l}- \\
-\end{array}$ & 0.9235 & 1.7527 & 18 \\
\hline Greece & $\begin{array}{l}0.3007 \\
(0.000)\end{array}$ & $\begin{array}{l}0.8689 \\
(0.000)\end{array}$ & $\begin{array}{l}0.1795 \\
(0.000)\end{array}$ & $\begin{array}{c}-0.2690 \\
(0.000)\end{array}$ & $\begin{array}{l}-0.0025 \\
(0.0002)\end{array}$ & $\begin{array}{l}-0.4537 \\
(0.0012)\end{array}$ & 0.9681 & 1.8727 & 19 \\
\hline Spain & $\begin{array}{l}-0.0662 \\
(0.6838)\end{array}$ & $\begin{array}{l}0.8898 \\
(0.000)\end{array}$ & $\begin{array}{l}-0.0642 \\
(0.5635)\end{array}$ & $\begin{array}{c}-0.9246 \\
(0.000)\end{array}$ & $\begin{array}{l}0.00294 \\
(0.3641)\end{array}$ & $\begin{array}{l}- \\
-\end{array}$ & 0.9042 & 2.0550 & 20 \\
\hline France & $\begin{array}{l}0.4518 \\
(0.000)\end{array}$ & $\begin{array}{c}0.4835 \\
(0.0005)\end{array}$ & $\begin{array}{c}0.2003 \\
(0.0001)\end{array}$ & $\begin{array}{c}-0.5440 \\
(0.000)\end{array}$ & $\begin{array}{l}-0.0069 \\
(0.0001)\end{array}$ & $\begin{array}{c}0.1954 \\
(0.14)\end{array}$ & 0.7160 & 1.8995 & 19 \\
\hline Italy & $\begin{array}{l}0.2417 \\
(0.000)\end{array}$ & $\begin{array}{l}0.6949 \\
(0.000)\end{array}$ & $\begin{array}{l}0.1082 \\
(0.000)\end{array}$ & $\begin{array}{l}-0.2634 \\
(0.0001)\end{array}$ & $\begin{array}{c}-0.0022 \\
(0.000)\end{array}$ & $\begin{array}{l}-0.5632 \\
(0.0006)\end{array}$ & 0.8501 & 1.2554 & 18 \\
\hline Netherlands & $\begin{array}{c}0.1613 \\
(0.0011)\end{array}$ & $\begin{array}{l}0.6093 \\
(0.000)\end{array}$ & $\begin{array}{l}0.0703 \\
(0.003)\end{array}$ & $\begin{array}{c}-0.4437 \\
(0.000)\end{array}$ & $\begin{array}{l}-0.0021 \\
(0.0065)\end{array}$ & $\begin{array}{c}-0.6262 \\
(0.000)\end{array}$ & 0.8866 & 1.8629 & 15 \\
\hline Austria & $\begin{array}{l}-0.0729 \\
(0.1884)\end{array}$ & $\begin{array}{l}-0.4231 \\
(0.0035)\end{array}$ & $\begin{array}{l}-0.1134 \\
(0.0001)\end{array}$ & $\begin{array}{l}-0.0456 \\
(0.5477)\end{array}$ & $\begin{array}{l}0.00281 \\
(0.0047)\end{array}$ & $\begin{array}{c}0.5782 \\
(0.0001)\end{array}$ & 0.0086 & 1.6340 & 17 \\
\hline Portugal & $\begin{array}{l}-0.0174 \\
(0.8033)\end{array}$ & $\begin{array}{l}0.9083 \\
(0.000)\end{array}$ & $\begin{array}{l}-0.0027 \\
(0.9413)\end{array}$ & $\begin{array}{l}-0.1123 \\
(0.2084)\end{array}$ & $\begin{array}{c}0.00218 \\
(0.1351)\end{array}$ & $\begin{array}{c}0.2619 \\
(0.1788)\end{array}$ & 0.9429 & 2.0453 & 18 \\
\hline Finland & $\begin{array}{l}-0.0242 \\
(0.5765)\end{array}$ & $\begin{array}{r}0.8346 \\
(0.000)\end{array}$ & $\begin{array}{l}-0.0369 \\
(0.0946)\end{array}$ & $\begin{array}{l}-0.2649 \\
(0.0006)\end{array}$ & $\begin{array}{c}0.0009 \\
(0.3362)\end{array}$ & $\begin{array}{l}-0.4350 \\
(0.0136)\end{array}$ & 0.4798 & 1.6373 & 16 \\
\hline Sweden & $\begin{array}{l}0.6628 \\
(0.000)\end{array}$ & $\begin{array}{c}0.17947 \\
0.2021\end{array}$ & $\begin{array}{l}0.5139 \\
(0.000)\end{array}$ & $\begin{array}{l}-0.297 \\
(0.000)\end{array}$ & $\begin{array}{c}0.0045 \\
(0.0014)\end{array}$ & $\begin{array}{c}0.4434 \\
(0.0043)\end{array}$ & 0.9191 & 1.6718 & 16 \\
\hline UK & $\begin{array}{c}0.0524 \\
(0.3184)\end{array}$ & $\begin{array}{c}0.4830 \\
(0.0021)\end{array}$ & $\begin{array}{c}0.0186 \\
(0.5621)\end{array}$ & $\begin{array}{c}-0.2401 \\
(0.000)\end{array}$ & $\begin{array}{c}0.0019 \\
(0.3178)\end{array}$ & $\begin{array}{c}0.5377 \\
(0.0005)\end{array}$ & 0.9078 & 2.0917 & 14 \\
\hline
\end{tabular}

Notes: The $p$-values are in parentheses. Results show the estimation of Eqs. (2.A.30) and (2.A.31). Total observations: 244. Sample period: 1994-2013. For Italy, the Netherlands, Portugal, and UK we use $\mathrm{AR}(2)$. For Ireland and Spain $\mathrm{AR}(1)$ and $\mathrm{AR}(2)$ terms are dropped because of insignificance.

and Germany. There is a significantly positive time trend for Austria and Sweden and a significantly negative one in Ireland, Greece, France, Italy, and the Netherlands. ${ }^{36}$ Intercepts are indeed different across countries and insignificant for Belgium, Spain, Austria, Portugal, Finland, and UK. In terms of adjusted R-squared, our equation performs badly only for Austria, probably because the labor market is strongly managed by country-specific institutions. In terms of serial correlation, Germany and Italy deviate most from the ideal value of two. Taking into account serial correlation has hardly any impact on the over-qualification results, but more so for the time trend. ${ }^{37}$

\section{A.3 Estimation of the role of institutions on the relationship between unemployment and downgrading}

To estimate the importance of labor market institutions regarding the impact of medium-skilled downgrading on low-skilled unemployment, we use the estimated coefficients of over-qualification from

have unit roots and their growth rates are stationary.

${ }^{36}$ When regressing over-qualification on a time trend we find highly significant positive coefficients, except for Denmark. However, when adding a lagged dependent variable the time trend becomes insignificant except for five of the countries and the lagged dependent variable has a significantly positive sign except for Belgium and France.

${ }^{37}$ It is worth mentioning that a full empirical analysis requires further robustness checks, such as testing different specifications or alternative estimation approaches (e.g. cross-section panel analysis); one has to avoid heterogeneity bias then. We deliberately leave a full-fledged empirical analysis to future research. 
2.A.3 Estimation of the role of institutions on the relationship between unemployment and downgrading

Table 2.5, column (4), to represent the latter impact. The nature of labor market institutions is captured by the net replacement rate. ${ }^{38}$

Table 2.6: Correlation between net replacement rate and the estimated unemployment-downgrading relationship

\begin{tabular}{lcccc}
\hline \hline Variable & Coefficient & Std. Error & t-Statistic & P-Value \\
\hline Const. & -0.344772 & 0.152286 & -2.263973 & 0.0470 \\
NRR & 0.006321 & 0.002884 & 2.191580 & 0.0532 \\
\hline \hline
\end{tabular}

Notes: $R^{2}$ and adj. $R^{2}$ are 0.72583 and -0.020159 ,

respectively; Observations $=12$.

The OLS-results are presented in Table 2.6 and show a significant positive relationship, indicating that downgrading is positively related to the net replacement rate. The low adjusted R-square stems from the high coefficients of Sweden (0.5) and France (0.2) in the regressions of Table 2.5. If we take them out, the adj. R-square would increase to 0.18 , and the slope still remains significantly positive.

\footnotetext{
${ }^{38}$ We have taken out Greece and Italy because of their extremely low replacement rates $(0.23$ and 0.25 , respectively) compared to the values between 50 and $75 \%$, which are outliers at the end of the spectrum and would therefore change the sign.
} 


\section{B Calibration}

\section{B.1 Cobb-Douglas production technology}

The underlying model used for the simulation is the following. ${ }^{39}$

- Production technology in the manufacturing sector

$$
Y_{m}=K_{m}^{\nu} H_{m}^{\alpha} l_{M}^{1-\alpha-\nu}
$$

- Production technology in the service sector

$$
Y_{s}=K_{s}^{\eta} H_{s}^{\beta} l_{L}^{1-\beta-\eta}
$$

- Wage curves

$$
w_{M}=\Phi\left(\lambda_{m}, d_{M}\right) w_{L}
$$

under endogenous benefit scheme (subscript 1):

$$
w_{L}=\Psi_{1}\left(\lambda_{s}, u_{L}\right) w_{M}
$$

under exogenous benefit scheme (subscript 2):

$$
w_{L}=\Psi_{2}\left(\lambda_{s}, u_{L}\right) B_{L}
$$

where

$$
\begin{gathered}
\Phi(\cdot)=\frac{1}{1-\bar{\lambda}_{m} / d_{M}}, \\
\Psi_{1}(\cdot)=\frac{1}{1+\frac{1-\xi}{\xi \kappa}-\frac{\bar{\lambda}_{s}}{\xi \kappa u_{L}}}, \\
\Psi_{2}(\cdot)=\frac{1}{1-\frac{\bar{\lambda}_{s}}{u_{L}}}, \\
\lambda_{m}=\frac{\delta_{m}}{(1-\alpha-\nu)\left(\sigma_{x}-1\right)}, \bar{\lambda}_{m}=\frac{\lambda_{m}}{1+\lambda_{m}} \\
\delta_{s}=\frac{\lambda_{s}}{1+\lambda_{s}}
\end{gathered}
$$

A rigorous discussion of the properties of the two wage curves and the uniqueness of the wage equilibrium is provided by 2.C. The rest of the model remains as discussed in the main text.

The capital accumulation follows Kaldor's facts, where in the long-run the rate of return to capital is constant as the capital stock adjusts perfectly.

\footnotetext{
${ }^{39}$ Notice that this assumption can be seen as the special case of the more generalized CES production function, i.e., $\sigma_{j} \rightarrow 1$, and by defining $H_{j}$ as a capital input composite produced with a Cobb-Douglas technology.
} 


\section{B.2 Benchmark statistics and calibration}

In order to provide a numerical solution of the model, we match the theoretical model with the data for a certain period. In doing so, we define the values for the production side, such as the input shares, as well as for the variables and parameters of our labor market, such as unemployment rates and skill downgrading rates. The parameters are given by $\left(\alpha, \beta, \nu, \eta, \rho, \sigma_{x}, \kappa, \xi, \delta_{m}, \delta_{s}, \lambda_{m}, \lambda_{s}\right)$. The endogenous variables are $\left(H_{m}, H_{s}, l_{M}, l_{L}, u_{L}, d_{M}, w_{H}, w_{M}, w_{L}, s_{L}, h, Y_{m}, Y_{s}, P_{m}, P_{s}\right)$ with the following exogenous variables $\left(N_{H}, N_{M}, N_{L}\right)$. We compute the values mostly from the EUKLEMS database. ${ }^{40}$ We also use when necessary different sources to obtain the values for the specific labor market parameters and variables. Table 2.7 provides an overview of the calibrated and benchmark equilibrium values. Note that, in order to have the best fit of the model with the data, we define the cost shares of the specific input factors simply as the sum of the cost shares of the low- and medium-skilled workers in each sector. Table 2.8 further summarizes the labor market benchmark values.

Table 2.7: Calibrated and benchmark equilibrium values for the industries

\begin{tabular}{|c|c|c|c|}
\hline \multirow[t]{2}{*}{ Description } & \multirow[t]{2}{*}{ Parameter/Variable } & \multicolumn{2}{|c|}{ Values } \\
\hline & & Germany & France \\
\hline Manuf. value-add (in 1000 Euros) ${ }^{(a)}$ & $P_{m} Y_{m}$ & 583,191 & 311,627 \\
\hline Service value-add (in 1000 Euros) ${ }^{(a)}$ & $P_{s} Y_{s}$ & $1,393,790$ & $1,142,089$ \\
\hline high-skilled labor force (in 1000 persons) ${ }^{(a)}$ & $N_{H}$ & 3,837 & 3,668 \\
\hline medium-skilled labor force (in 1000 persons) ${ }^{(a)}$ & $N_{M}$ & 24,148 & 15,782 \\
\hline low-skilled labor force (in 1000 persons) ${ }^{(a)}$ & $N_{L}$ & 10,022 & 4,622 \\
\hline Total labor force & $N=N_{H}+N_{M}+N_{L}$ & 38,007 & 24,071 \\
\hline medium-skilled wage rate ${ }^{(a)}$ & $w_{M}$ & 30.15 & 23.65 \\
\hline low-skilled wage rate ${ }^{(a)}$ & $w_{L}$ & 21.65 & 20.18 \\
\hline Manufacturing capital cost share ${ }^{(a)}$ & $\nu$ & 0.27 & 0.31 \\
\hline Manuf. high-skilled cost share ${ }^{(a)}$ & $\alpha$ & 0.08 & 0.06 \\
\hline Manuf. medium-skilled cost share ${ }^{(a)}$ & $1-\alpha-\nu$ & 0.65 & 0.63 \\
\hline Service capital cost share ${ }^{(a)}$ & $\eta$ & 0.38 & 0.36 \\
\hline Serv. high-skilled cost share ${ }^{(a)}$ & $\beta$ & 0.11 & 0.18 \\
\hline Serv. low-skilled cost share ${ }^{(a)}$ & $1-\beta-\eta$ & 0.51 & 0.46 \\
\hline Unemployment rate $^{(b)}$ & $u_{L}$ & 0.15 & 0.13 \\
\hline Skill downgrading rate ${ }^{(b)}$ & $d_{M}$ & 0.255 & 0.33 \\
\hline \multirow[t]{2}{*}{ Elasticity of Substitution ${ }^{(c)}$} & $\sigma_{x}=\frac{1}{1-\rho}$ & 4 & 4 \\
\hline & $\rho$ & 0.75 & 0.75 \\
\hline Net replacement rate ${ }^{(d)}$ & $\xi$ & 0.58 & 0.58 \\
\hline
\end{tabular}

(a) EUKLEMS. Own calculation.

(b) Eurostat.

(c) From Abraham et al. (2009).

(d) OECD Tax \& Benefits Statistics.

\footnotetext{
${ }^{40}$ For an extensive description of the data and the calibration approach, we refer the reader to the working paper version which will be provided upon request.
} 
Table 2.8: Labor market benchmark values

\begin{tabular}{|c|c|c|c|}
\hline \multirow[t]{2}{*}{ Description } & \multirow[t]{2}{*}{ Parameter/Variable } & \multicolumn{2}{|c|}{ Value } \\
\hline & & Germany & France \\
\hline Manufacturing high-skilled employment (in 1000)(e) & $H_{m}$ & 850 & 312 \\
\hline \multirow[t]{2}{*}{ Service high-skilled employment (in 1000) } & $H_{s}=N_{H}-H_{m}$ & 2,987 & 3355 \\
\hline & $h=\frac{H_{m}}{N_{H}}$ & 0.22 & 0.085 \\
\hline medium-skilled employment (in 1000) & $l_{M}=\left(1-d_{M}\right) N_{M}$ & 17,990 & 10,574 \\
\hline \multirow[t]{2}{*}{ low-skilled employment (in 1000) } & $l_{L}=\left(1-u_{L}\right) N_{L}+d_{M} N_{M}$ & 14,676 & 9,229 \\
\hline & $l=\left(1-u_{L}\right) N_{L} / L$ & 0.58 & 0.44 \\
\hline high-skilled wage rate $(f)$ & $w_{H}=\alpha P_{m} Y_{m} / H_{m}$ & 51.82 & 61.37 \\
\hline \multirow[t]{2}{*}{ Manufacturing trade union bargaining power ${ }^{(g)}$} & $\delta_{m}$ & 0.1265 & 0.0853 \\
\hline & $\lambda_{m}$ & 0.0774 & 0.0508 \\
\hline \multirow[t]{2}{*}{ Service trade union bargaining power ${ }^{(h)}$} & $\delta_{s}$ & 0.0669 & 0.0715 \\
\hline & $\lambda_{s}$ & 0.0481 & 0.0578 \\
\hline Weighting factor ${ }^{(i)}$ & $\kappa$ & 0.50 & - \\
\hline Manufacturing-wage curve & $\Phi(\cdot)$ & 1.39 & 1.17 \\
\hline Services-wage curve & $\Psi(\cdot)$ & 0.72 & 1.72 \\
\hline Elasticity of the manufacturing-wage curve & $\varepsilon_{M}=\frac{\partial \log \Phi(\cdot)}{\partial \log d_{M}}$ & -0.39 & -0.17 \\
\hline Elasticity of services-wage curve & $\varepsilon_{L}=\frac{\partial \log \Psi(\cdot)}{\partial \log u_{L}}$ & -0.76 & -0.72 \\
\hline
\end{tabular}

(e) Calibration is based on the condition $\alpha \frac{P_{m} Y_{m}}{H_{m}}=w_{H}=\beta \frac{P_{s} Y_{s}}{N_{H}-H_{m}}$.

( $f$ ) Calibration is based on values in Table 2.7.

(g) Calibrated from the manufacturing wage-setting curve (2.B.34).

(h) Calibrated from the services wage-setting curve (2.B.35) and (2.B.36).

(i) Assumption. 


\section{C Bargaining solution and unique equilibrium}

\section{C.1 Bargaining solution}

Recall the Nash bargaining problem

$$
\max _{w_{i}}\left\{\left(\left(w_{i}-\bar{w}_{i}\right) l_{i}\right)^{\delta_{j}} \Pi_{j}^{1-\delta_{j}}\right\}
$$

Take logs and differentiate w.r.t. $w_{i}$ to obtain

$$
\delta_{j} \frac{1}{w_{i}-\bar{w}_{i}}+\delta_{j} \frac{\partial l_{i}}{\partial w_{i}} \frac{1}{l_{i}}+\left(1-\delta_{j}\right) \frac{\partial \Pi_{j}}{\partial w_{i}} \frac{1}{\Pi_{i}}=0
$$

Multiplying the first order condition (2.C.38) by $w_{i}$ and rearranging yields

$$
\delta_{j} \frac{w_{i}}{w_{i}-\bar{w}_{i}}=-\delta_{j} \frac{\partial l_{i}}{\partial w_{i}} \frac{w_{i}}{l_{i}}-\left(1-\delta_{j}\right) \frac{\partial \Pi_{j}}{\partial w_{i}} \frac{w_{i}}{\Pi_{i}} .
$$

By the Envelope Theorem,

$$
\frac{\partial \Pi_{j}}{\partial w_{i}}=-l_{i} .
$$

Thus, the term

$$
\frac{\partial \Pi_{j}}{\partial w_{i}} \frac{w_{i}}{\Pi_{i}}=-\frac{w_{i} l_{i}}{\Pi_{j}}
$$

denotes the cost share of labor input in firm's flow profit. In the general equilibrium context, i.e., taking account of goods demand effects, this is given by

$$
\frac{w_{i} l_{i}}{\Pi_{j}}=\left(\sigma_{x}-1\right) \theta_{i} .
$$

with $\theta_{i}$ denoting the cost share of labor input.

The first term in the right-hand side of $\left(2 . \mathrm{C} .38^{\prime}\right), \frac{\partial l_{i}}{\partial w_{i}} \frac{w_{i}}{l_{i}}$, denotes the labor demand elasticity. In the general equilibrium context, this is given

$$
\frac{\partial l_{i}}{\partial w_{i}} \frac{w_{i}}{l_{i}}=-\left(\sigma_{x}-\sigma_{j}\right) \theta_{i} .
$$

Utilizing (2.C.39) and (2.C.40) in (2.C.38') and rearranging, yields the standard wage markup behaviour derived in the main text.

\section{C.2 Unique equilibrium condition under endogenous benefits}

We assume that the level of unemployment benefits is tied closely to the average wage. In particular, $B_{L}$ is defined as a percentage $(\xi)$ of the average of the low- and medium-skilled wages weighted by $\kappa:{ }^{41}$

$$
B_{L}=\xi\left(\kappa w_{M}+(1-\kappa) w_{L}\right) .
$$

\footnotetext{
${ }^{41}$ Note that for $\kappa=0$ we obtain the standard definition of unemployment benefits as the constant "replacement rate", $b / w_{L}=\xi$. In this case, however, the linkage between $w_{L}$ and $w_{M}$ disappears and the services-wage curve will be defined as $w_{L}=\left(1+\lambda_{s}\right)\left(\left(1-u_{L}\right) w_{L}+u_{L} b\right)$. Consequently, the equilibrium unemployment rate, $u_{L}$, will be constant and depend only on the parameters of the model. This, in turn, implies that no longer reacts to migration shocks.
} 
Using the definition for $B_{L}$ (2.C.41) in Eq. (2.16) and rearranging, we obtain the wage curve (2.B.35). Note that if the service labor union loses bargaining power, then the perfect competition outcome with no unemployment results, i.e., if $\delta_{s} \rightarrow 0$, then $\lambda_{s} \rightarrow 0$ and $w_{L} \rightarrow B_{L}$. Moreover, from Eq. (2.B.35) it follows that the higher is the weighting parameter $\kappa$, the stronger is the linkage to the medium-skilled wage, $\frac{\partial \Psi_{1}}{\partial \kappa}>0$. Thus, we conclude

Proposition 2.3. A stronger linkage between low- and medium-skilled workers due to wage bargaining and endogenous unemployment benefits induces wage compression at the lower end of the wage distribution.

This is in line with the cross-firm evidence for Germany where the wage differential between lowand medium-skilled workers in unionized firms is lower than for non-unionized firms (Dustmann and Schönberg, 2009). ${ }^{42}$

\section{Properties of the manufacturing wage curve}

The partial features of the manufacturing-wage curve (i.e., taking $w_{L}$ as given), can be demonstrated as follows. For a better realization, rewrite Eq. (2.B.34) as

$$
\frac{w_{L}}{w_{M}}=\Phi\left(\lambda_{m}, d_{M}\right)^{-1} \equiv 1-\frac{\bar{\lambda}_{m}}{d_{M}\left(w_{M}\right)},
$$

where $\bar{\lambda}_{m}=\frac{\lambda_{m}}{1+\lambda_{m}}$ and $d_{M}\left(w_{M}\right)$ is given by Eq. (2.11). Now, the LHS and RHS can both be seen as functions of $w_{M}$ for given values of $w_{L}$. This is because manufacturing unions take the outside option $\left(w_{L}\right)$ as given when they negotiate. Then, it can be easily verified that the LHS of Eq. (2.C.42) is a decreasing function of $w_{M}$ but the RHS is increasing for certain values of both $o_{M}$ and $w_{M}$. These relations are illustrated in Figure 2.2.

\section{Figure 2.2: Properties of the manufacturing-wage curve}

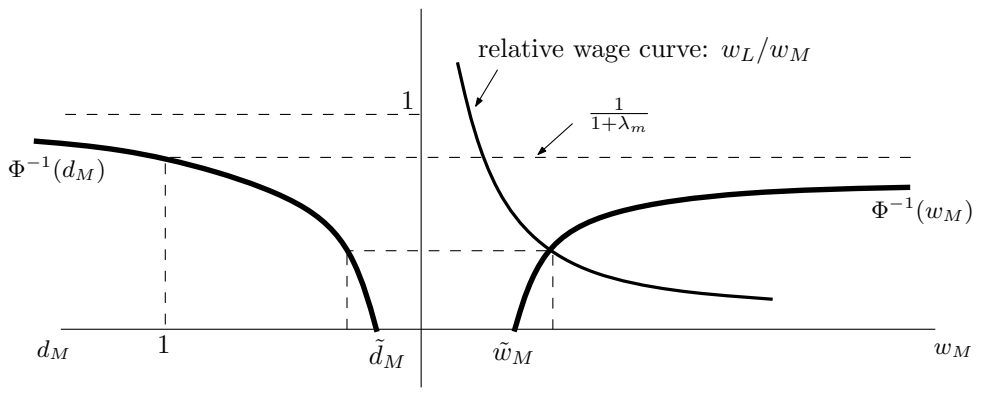

Consider first the right panel of Figure 2.2. Then, recalling (2.C.42), we can draw two curves: one shows the negative relation between the relative wage rate due to changes in $w_{M}$, the LHS of (2.C.42), while holding the low-skilled wage fixed; the second curve illustrates the positive relation between the inverse-wage-mark-up function, $\Phi^{-1}$, and the medium-skilled wage rate $w_{M}$. This relation follows from the positive relation between the skill downgrading rate and the medium-skilled wage rate as higher wages induces a decline in the demand for labor and thus increase the risk of downgrading. Recalling the medium-skilled labor demand (2.6.1) and the skill downgrading rate (2.11), then, one can compute the limiting cases

$$
\lim _{w_{M} \rightarrow \infty} l_{M}^{d}=0 \Rightarrow \lim _{w_{M} \rightarrow \infty} d_{M}=1 \Rightarrow \lim _{w_{M} \rightarrow \infty} \Phi^{-1}=\frac{1}{\lambda_{m}} .
$$

\footnotetext{
${ }^{42}$ Computing the effects of different values for $\kappa$, we find that a stronger linkage between low- and medium-skilled workers (i.e., $\kappa \rightarrow 1$ ), reduces the magnitude of changes in the relation between low- and medium-skilled wages.
} 
The intersection between the two curves in the right plane will determine the equilibrium skill downgrading rate and the medium-skilled wage rate for changes in the low-skilled wage rate. We conclude Lemma 2.1. Positive wages are ensured iff $d_{M} \in\left(\tilde{d}_{M}, 1\right)$.

Proof. The proof is rather straightforward. Due to the non-negativity assumption of the wage rates, it follows from Eq. (2.C.42) that

$$
\begin{aligned}
\Phi^{-1} & >0 \\
\frac{\bar{\lambda}_{m}}{d_{M}} & <1 \\
d_{M} & >\tilde{d}_{M} \equiv \frac{\lambda_{m}}{1+\lambda_{m}} .
\end{aligned}
$$

This defines the lower boundary of the skill downgrading rate.

\section{Properties of the services wage curve}

Similarly, the partial behaviour of the services-wage curve can be assessed as follows. First, rewrite (2.B.35) as

$$
\frac{w_{M}}{w_{L}}=\Psi_{1}\left(\lambda_{s}, u_{L}\right)^{-1} \equiv 1+\frac{1-\xi}{\xi \kappa}-\frac{\bar{\lambda}_{s}}{\xi \kappa u_{L}},
$$

where $\bar{\lambda}_{s}=\frac{\lambda_{s}}{1+\lambda_{s}}$. With the same intention describing the manufacturing wage curve, we define both the LHS and RHS of Eq. (2.C.43) as functions of $w_{L}$ for given values of $w_{M}$. The argumentation is analogous to that for the manufacturing-wage curve. Thus, we can define two curves with opposite relations to changes in $w_{M}$ as shown in the right plane of Figure 2.3, whereas the left plane shows the relation between $\Psi^{-1}$ and the unemployment rate $u_{L}$.

Figure 2.3: Properties of the services-wage curve

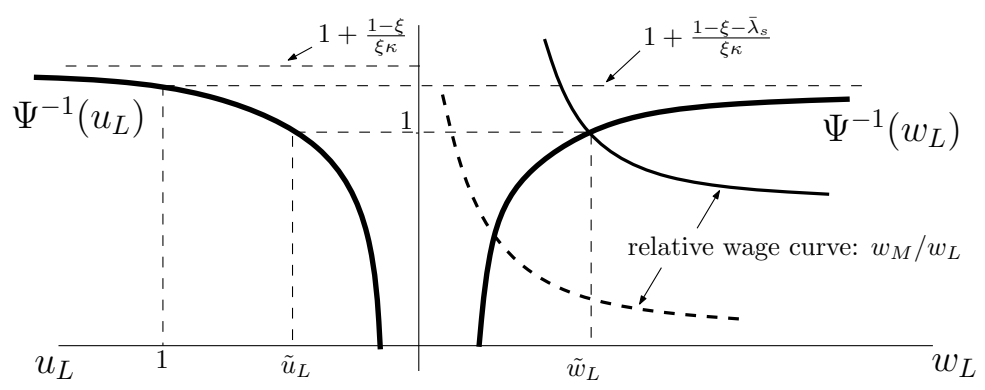

However, the condition that must be satisfied in this case is summarized by the following lemma.

Lemma 2.2. The relation $w_{M}>w_{L}$ is ensured iff $u_{L} \in\left(\tilde{u}_{L}, 1\right)$.

Proof. From Eq. (2.C.43), it follows that

$$
\begin{aligned}
\Psi^{-1} & >1 \\
\frac{\bar{\lambda}_{s}}{u_{L}} & <1-\xi \\
u_{L} & >\tilde{u}_{L} \equiv \frac{\bar{\lambda}_{s}}{1-\xi} .
\end{aligned}
$$


This implies that for values of the unemployment rate $u_{L} \in\left(0, \tilde{u}_{L}\right]$ the relation between the low- and medium-skilled wage rates is violated, i.e., $w_{M} \leq w_{L}$. Therefore, to ensure $w_{M}>w_{L}$, the unemployment rate must be strictly larger than the lower boundary $\tilde{u}_{L}$.

Now, from these conditions, the unique intersection of the two wage-setting curves can be shown graphically in the $\left(w_{M}, w_{L}\right)$-plane. By Lemma 2.1 and $2.2, w_{M}>w_{L}>0$. This indicates that in the $\left(w_{M}, w_{L}\right)$-space the wage relation should always be above the 45 degree line. Starting with $W C_{M}$, one sees from the RHS plane of Figure 2.2 that for large values of the low-skilled wage rate, the mediumskilled equilibrium wage rises along the $\Phi^{-1}$ curve due to upward shifts of the relative wage curve. Hence,

Figure 2.4: Unique equilibrium

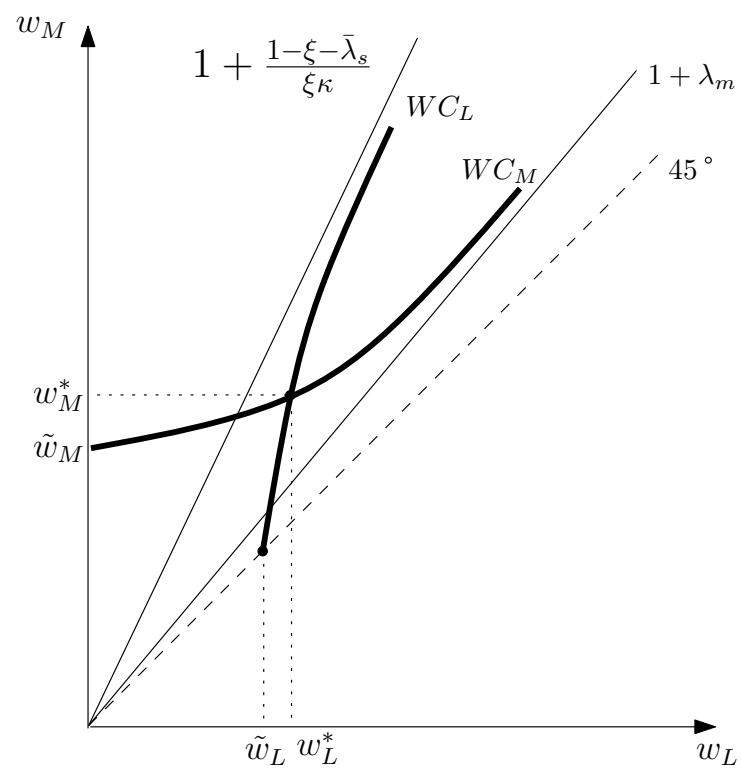

a higher $w_{L}$ increases the equilibrium $w_{M}$ and with it the skill downgrading rate, which converges to $1+\lambda_{m}$, the reciprocal of the limit shown in Figure 2.2.

Analogously, the derivation of $W C_{L}$ can be explained by recalling the RHS of Figure 2.3. Now, changes in $w_{M}$ are associated with moving along the $\Psi^{-1}$ curve. However, as explained above, the necessary condition requires that $\Psi^{-1}>1$ for $w_{L}>\tilde{w}_{L}$. This indicates that in $\left(w_{M}, w_{L}\right)$-space the $W C_{s}$ must start above the 45 degree line. As described above, a higher $w_{M}$ leads to a higher $w_{L}$ along the $\Psi^{-1}$ curve, converging to the limit $1+\frac{1-\xi-\bar{\lambda}_{s}}{\xi \kappa}$. Notice that in the $\left(w_{M}, w_{L}\right)$-plane, the inverse services-wage curve is drawn. To ensure a unique equilibrium, $1+\frac{1-\xi-\bar{\lambda}_{s}}{\xi \kappa}>1+\lambda_{m}$ must hold, which leads to the following lemma.

Lemma 2.3. A unique intersection between the two wage curves is ensured for all

$$
\xi<\tilde{\xi} \equiv \frac{1}{1+\lambda_{s}} \frac{1}{1+\kappa \lambda_{m}} .
$$

In Table 2.9, we summarize these conditions and assume that they hold. ${ }^{43}$

An illustration of the interdependence relation reveals that a productivity improvement in the manufacturing sector, relative to the service sector, increases the low-skilled wage rate in the service

\footnotetext{
${ }^{43}$ In the calibration of the model we show that these conditions do hold for plausible parameter values consistent with the empirics-see 2.B.
} 
Table 2.9: Equilibrium Conditions

\begin{tabular}{lll}
\hline \hline Parameter/Variable & Range & Condition \\
\hline$d_{M}$ & $\in\left(\tilde{d}_{M}, 1\right)$ & Lemma 2.1 \\
$w_{M}$ & $>\tilde{w}_{M}$ & \\
\hline$u_{L}$ & $\in\left(\tilde{u}_{L}, 1\right)$ & Lemma 2.2 \\
$w_{L}$ & $>\tilde{w}_{L}$ & \\
\hline$\xi$ & $<\tilde{\xi}$ & Lemma 2.3 \\
\hline \hline
\end{tabular}

sector without any justification by a corresponding productivity increases in the latter. This phenomenon is also widely recognized as the main cause of the so-called Baumol's disease - see, for instance, Hartwig (2011). It also corresponds to the observation that the low wage differentiation in Continental Europe is attributed to the centralization and coordination of wage formation (Siebert, 1997). 


\section{3}

\section{Offshoring of Medium-skill Jobs, Polarization, and Productivity Effect: Implications for Wages and Low-skill Unemployment ${ }^{1}$}

\subsection{Introduction}

One key feature of recent globalization trends is the growing phenomenon of international reorganization of production and work processes, or, put differently, offshoring of jobs, which has heightened concerns regarding job and wage cuts in many advanced countries (cf. Bhagwati et al., 2004; Snower et al., 2009). ${ }^{2}$ While earlier studies have highlighted the labor market impact of international fragmentation of the value added chain, captured by the increasing penetration of intermediate goods (Feenstra and Hanson, 1996, 1999; Jones and Kierzkowski, 1990, 2001; Kohler, 2004a,b), recent observations accentuate the important role of job characteristics and task content of occupations (cf. Blinder, 2009a,c). To put it in the words of Blinder (2009c, p.54), "... this time it's not the British who are coming, but the Indians. . neither by land nor by sea, but electronically".

More precisely, the recent development in employment and wages depicts a polarizing

\footnotetext{
${ }^{1}$ This chapter is based on joint work with Joan Muysken and Thomas Ziesemer, and is published in IAB-Discussion Paper 7/2015 (Vallizadeh et al., 2015). We are grateful for valuable comments and discussions to Hartmut Egger, Ingo Geishecker, Francesc Ortega and to participants at the $2^{\text {nd }}$ International IAB/RCEA/ZEW Workshop in Nuremberg, 2014, at the $3^{\text {rd }}$ Joint Workshop of Aarhus University and IAB in Nuremberg, 2014, and at ESPE 2014 in Braga.

${ }^{2}$ Blinder (2009a) estimates that 30 million to 40 million jobs in the USA are potentially offshorable, while job tasks that require face-to-face contact as well as abstract and cognitive skills are protected. See also the studies by Jensen and Kletzer (2010) and Moncarz et al. (2008) regarding offshorability of service occupations. For example, Moncarz et al. (2008) identify the offshorability of 160 service occupations, where the range of occupations includes scientists, mathematicians, radiologists and editors at the high end of the market as well as those of telephone operators, clerks and typists at the low end.
} 
trend in many advanced countries, indicating a deflection of global competition towards the medium-skilled workforce (Acemoglu and Autor, 2011). ${ }^{3}$ The rationale for this new trend is, on the one hand, advances in information and communication technology (ICT), as well as declines in trade transaction and transportation costs of goods and services that have accelerated the integration process of national markets into a global market. On the other hand, an important factor is rapid growth in major emerging countries, such as Brazil, Russia, India, and China (BRIC). These countries are characterized by high accumulation of human-capital and advanced technologies as well as improvements in the economic and business infrastructure that make them highly competitive in areas such as information technology services in which the advanced countries have been dominant (Bhagwati et al., 2004; Snower et al., 2009; Spence, 2011). ${ }^{4}$ Both developments have reduced the locational viability of some occupations. That is, jobs that have a high content of routine, non-interactive, and non-cognitive tasks are most likely at peril. The rationale is that these routine-intensive job tasks can be easily codified, enabling firms in many advanced countries to reorganize production and work processes by decomposing the various stages of production geographically into clusters of tasks and locating each task cluster in the countries where it is most profitable (Snower et al., 2009). Therefore the comparative advantage of performing a specific type of job tasks has become important.

In the recent literature on offshoring job tasks, two main forces have received particular attention, introduced by Grossman and Rossi-Hansberg (2008) trade-in-tasks approach. ${ }^{5}$ While allocating jobs abroad (offshoring) induces a direct displacement effect of domestic workers, leading to lower wages, offshoring activities may generate a productivity effect similar to technology improvement by lowering a firm's production cost. This productivity effect, in turn, will lead to an expansion of output and thus raise employment and wages. The balance between these two forces will determine the direction of the wage and employment effect of offshoring (Baumgarten et al., 2013; Becker et al., 2013; Ebenstein et al., 2014; Harrison and McMillan, 2011; Hummels et al., 2014; Olney, 2012; Ottaviano et al., 2013; Wright, 2014).

However, there exists another important mechanism that shapes substantially the labor market outcomes of offshoring: the spillover effects on other skill groups induced by job tasks mobility of offshoring-induced displaced workers. This channel is omitted in the existing theoretical literature on offshoring and task allocation. Our first contribution is, therefore, to assess the two hypotheses regarding polarization and productivity effects in an integrated general framework that accounts explicitly for several important features: task-skill heterogeneity, endogenous offshoring, and spillover effects due to mobility across job tasks. In doing so, we build on recent important contributions that incorporate the

\footnotetext{
${ }^{3}$ For recent empirical evidence regarding the polarization effect in the US labor market see Autor and Dorn (2009, 2013); Autor et al. (2006, 2008, 2003); Firpo et al. (2011); Michaels et al. (2014); and in the European labor markets Baumgarten et al. (2013); Dustmann et al. (2009); Goos and Manning (2007); Goos et al. (2009, 2014); Spitz-Oener (2006).

${ }^{4}$ More specifically, Bhagwati et al. (2004) highlight that the stock of skilled workers in India and China will reach about 300 million in a few decades. Spence (2011) provides a critical discussion on globalization and labor market effects for the USA.

${ }^{5}$ A third channel, as put forward in Grossman and Rossi-Hansberg (2008), is via the terms-of-trade effect that may wipe out the productivity effect. However, see Bhagwati et al. (2004) for a discussion regarding the empirical insignificance of terms-of-trade effects of offshoring.
} 
explicit assignment of skills to tasks. ${ }^{6}$ In particular, we provide a theoretical framework that augments recent contributions by Acemoglu and Autor (2011), Grossman and RossiHansberg (2008), and Ottaviano et al. (2013) in two ways. First, we allow for endogenous specialization of different skill groups into a continuum of tasks. The implied matching between tasks and skills allows for task competition among different skill groups and thus enables us to jointly investigate changes in the task composition performed by different skills in the economy due to exogenous offshoring shocks, a feature that is absent in Grossman and Rossi-Hansberg (2008) and Ottaviano et al. (2013). ${ }^{7}$ Second, and contrary to Acemoglu and Autor (2011), who consider offshoring exogenously, we analyze the offshoring decision by domestic firms as an endogenous process, capturing changes at both the intensive margin, i.e. the range of offshorable tasks, as well as the extensive margin, i.e. the offshorability of a marginal domestic task.

Thus our theoretical framework accounts for all these different mechanisms, which have been addressed separately in the previous literature. Particularly, several important insights can be gained from our approach. First, we show that any offshoring scenario of domestic job tasks can be described by a non-monotonic, U-shaped task productivity schedule. This permits us to capture various phases of international competition and to address their distributional effects for the domestic workforce. As we elaborate below, if offshoring activities are limited to low-skilled job tasks - depicting globalization trends in the past (cf. Snower et al., 2009) - then lower offshoring frictions generate a distributional effect similar to skill-biased technology changes. If, on the other hand, offshoring is limited to high-skilled job tasks, then easier offshoring induces wage impacts similar to unskill-biased technology changes.

Second, we capture three main channels characterizing the recent phase of globalization: i) accumulation of advanced technologies abroad, i.e. a technology effect, ii) accumulation of human capital abroad, i.e. a endowment effect, and iii) decline in transport barriers, i.e. a trade cost effect. Although qualitatively they generate similar effects, accounting explicitly for them permits us to address not only changes in the nature of North-South trade, i.e. trade in goods and services between "rich" and "poor" countries, where trade barriers are still substantial, but also the implications of market integration, such as in the context of the enlargement of the European Union towards Central, Eastern, and South-Eastern European countries since 2004, where trade costs are effectively null, but changes in comparative advantages are characterized by a rapid accumulation of advanced technologies and human capital in these regions.

Another new insight, gained from the general equilibrium analysis, is that the costefficiency effect induced by easier offshoring depends now on the magnitude of the spillover effects. On the one hand, easier offshoring induces a reallocation of tasks performed by medium-skilled workers to offshore workers. This external reallocation is the main source

\footnotetext{
${ }^{6}$ See Acemoglu and Autor (2011) for an elaborate discussion on the limitation of the standard nested CES (so-called "canonical") model compared to the "task-assignment" approach. For the alternative task-skill-assignment approach, see also Autor and Dorn (2013); Costinot and Vogel (2010); Dupuy (2012); Sly (2012).

${ }^{7}$ Notice the important difference to Ottaviano et al. (2013), who assess the task allocation between immigrant, offshore and native workers, though each factor is homogeneous in terms of skills. Our framework, instead, could be easily extended to incorporate task competition between immigrants and natives, e.g. by a CES decomposition of factor labor per task.
} 
of the productivity effect due to lower offshoring cost. There is, on the other hand, a offshoring-induced spillover effect on other domestic skill groups, which we refer to as the domestic reallocation of workers, i.e. from medium-skilled to low- and high-skilled job tasks. This internal reallocation countervails the cost-efficiency effect induced by the external reallocation. Moreover, our analysis reveals that the difference between these two forces depends crucially on the elasticity of substitution between skill groups at the respective extensive task margins.

The importance of this internal reallocation for the labor market impact of offshoring has been put forward in several recent empirical studies (Baumgarten et al., 2013; Ebenstein et al., 2014; Hummels et al., 2014). In a nutshell, the empirical evidence suggests that switching occupation is costly for offshoring-induced displaced workers. Using matched worker-firm data from Denmark, Hummels et al. (2014) find that offshoring increases the skill premium within firms, i.e. the relative wage of skilled workers, and that the downward wage pressure is more pronounced in occupations that involve routine tasks. However, by allowing for labor mobility across occupations, they find that the cohortaverage wage loss (i.e. of workers who leave the firm, and those who stay) is exacerbated for both low- and high-skilled workers. The authors relate the latter outcome to losses in specific human capital and search cost that considerably hinder the reattachment to the labor market for the offshoring-induced displaced workers. Ebenstein et al. (2014) investigate the impact of trade and offshoring on wages for the USA. The empirical findings show that import penetration and offshoring induce a downward pressure for workers performing routine intensive occupations, while export activities have a positive impact. Moreover, the empirical evidence emphasizes that the negative wage effect becomes substantial once occupation-sector mobility of workers is taken into account, suggesting the important role of occupation-specific human capital. Using data for Germany, Baumgarten et al. (2013) find a substantial negative cross-industry wage pattern due to offshoring in occupations with a high routine task content relative to interactivity and non-routine content of occupational tasks, for both low-skilled and highskilled workers. Our framework contributes also to the empirical literature by providing structural guidance on the occupational mobility of displaced workers. As we discuss below, the degree of task substitutability between different skill groups is the critical parameter that accounts for the magnitude of internal reallocation.

Another particular debate on offshoring is the displacement effect of the least skilled workers from the labor market. We address this concern by assuming equilibrium unemployment of low-skilled workers in the economy. To keep the framework tractable, we allow for two types of labor market friction. The first source is given by a minimum wage scheme that is set above the market-clearing wage rate and thus leads to unemployment. In a second step, we allow for endogenous supply of low-skilled labor services. While the former - reflecting a perfectly elastic labor supply curve - corresponds to the mirror image of the full employment case, the latter facilitates a more general notion of how the labor market adjusts to offshoring shocks by allowing an elastic labor supply curve. In this second step we derive clear conditions under which the offshoring-induced spillover effects translate into higher low-skilled employment. This is the second contribution to the literature on offshoring tasks. ${ }^{8}$

\footnotetext{
${ }^{8}$ It is worth mentioning the study by Kohler and Wrona (2011), who introduce equilibrium unemployment following the search and matching theory and the efficiency wage theory into the
} 
The rest of this chapter is organized as follows. In the next section, we present the task assignment model. In section 3.3, we describe the production technology and derive the solution to the firm's optimization problem. Section 3.4 provides the general equilibrium solution followed by the comparative statics in section 3.5. The implications of offshoring and spillover effects on low-skill unemployment are discussed in section 3.6. Finally, section 3.7 offers concluding remarks.

\subsection{Task assignment}

In this section, we discuss the main properties of the task-based framework introduced by Acemoglu and Autor (2011) and Grossman and Rossi-Hansberg (2008). As will be seen below, the factor labor loses its static property and is now assigned to a continuum of tasks based on a Ricardian type of comparative advantage.

Consider an economy where different groups of workers perform a job task. The range of the existing tasks is defined over a unit interval. More specifically, a task $i$ can be performed in different modes: by domestic low- $(L)$, medium- $(M)$, and high-skilled $(H)$ labor, as well as by foreign, offshored workers $(O)$. Formally, the production function for a task is written as

$$
t(i)=A_{L} a_{L}(i) l_{L}(i)+A_{M} a_{M}(i) l_{M}(i)+A_{H} a_{H}(i) l_{H}(i)+A_{O} a_{O}(i) l_{O}(i) / \tau, \quad i \in[0,1],
$$

where $A$ denotes the factor-augmenting technology and $a(i)$ indicates the job-task productivity schedule of the respective labor type. The "iceberg" type offshoring friction is captured by the parameter $\tau \geq 1$. The number of low-, medium-, high-skilled and offshore workers performing a job-task $i$ is denoted by $l_{k}(i), k=\{L, M, H, O\}$.

Below we derive the optimal allocation between the three domestic skill groups as well as the offshore workers. While the single good producer solves simultaneously the optimal assignment of tasks to different labor groups, we demonstrate this procedure sequentially to make the optimal decision behavior better understandable.

\subsubsection{Allocation of domestic tasks}

We assume that, over the unit interval, tasks are ordered according to the skill requirement in a monotonic way. For example, one can think of this order as manual (e.g. restoring houses and servicing), routine (e.g. bookkeeping and running a machine), and cognitive (e.g. research and management activities) tasks.

Assumption 1. (Domestic Task Productivity)

For all $i \in[0,1], \frac{a_{L}(i)}{a_{M}(i)}$ and $\frac{a_{M}(i)}{a_{H}(i)}$ are strict monotonically decreasing in $i$, and by transitivity property $\frac{a_{L}(i)}{a_{H}(i)}$ is also strict monotonically decreasing in $i$.

original task framework of Grossman and Rossi-Hansberg (2008), characterized by a single sector and homogeneous factor labor. However, our objective is neither to compare different paradigms of labor market friction nor to discuss their different adjustment mechanisms. Thus we deliberately avoid any unnecessary complexity of the model. 
Since the firm allocates tasks to the group that is the most cost-efficient in performing those tasks, this assumption allows us to determine the equilibrium allocation of tasks among the domestic workers. Let the unit cost of producing task $i$ domestically be $c_{k}(i)=\frac{w_{k}}{A_{k} a_{k}(i)}, k=\{L, M, H\}$ where $w_{k}$ denotes the wage costs, and $c_{L}(0)<c_{M}(0)<$ $c_{H}(0)$. Denoting relative task-productivity by $\beta_{L}(i)=\frac{a_{L}(i)}{a_{M}(i)}$ and $\beta_{H}(i)=\frac{a_{M}(i)}{a_{H}(i)}$, then, in equilibrium, it follows

Lemma 3.1 (Domestic Task Allocation). By Assumption 1, there exist task margins $I_{L}, I_{H}$, and $\tilde{I}$, respectively, where a representative domestic firm is indifferent between

- Low- and medium-skilled workers

$$
\frac{w_{L}}{A_{L}}\left(\beta_{L}\left(I_{L}\right)\right)^{-1}=\frac{w_{M}}{A_{M}}
$$

- Medium- and high-skilled workers

$$
\frac{w_{H}}{A_{H}} \beta_{H}\left(I_{H}\right)=\frac{w_{M}}{A_{M}}
$$

- Low- and high-skilled workers

$$
\frac{w_{L}}{A_{L}}\left(\beta_{L}(\tilde{I})\right)^{-1}=\beta_{H}(\tilde{I}) \frac{w_{H}}{A_{H}} .
$$

Proof. See Appendix 3.B.1.

Thus, by Lemma 3.1, the domestic allocation of tasks to skill groups is characterized by the two endogenous thresholds, $I_{L}$ and $I_{H}$. Moreover, Eqs. (3.2) and (3.3) determine the degree of substitutability between the domestic skill groups. Put differently, the reallocation of medium-skilled workers to low- and high-skilled intensive job tasks depends on their comparative advantages in the neighborhood of $I_{L}$ and $I_{H}$ relative to low-skilled and high-skilled workers, which is characterized by $\beta_{L}(i)$ and $\beta_{H}(i)$. More formally,

$$
\begin{gathered}
\frac{\mathrm{d} I_{L}}{\mathrm{~d} \ln \left(w_{M} / w_{L}\right)}=\frac{1}{\varepsilon_{L}} \\
\frac{\mathrm{d} I_{H}}{\mathrm{~d} \ln \left(w_{H} / w_{M}\right)}=\frac{1}{\varepsilon_{H}},
\end{gathered}
$$

where $\varepsilon_{L}=-\frac{\partial \ln \beta_{L}\left(I_{L}\right)}{\partial I_{L}}>0$ and $\varepsilon_{H}=-\frac{\partial \ln \beta_{H}\left(I_{H}\right)}{\partial I_{H}}>0$ denote the semi-elasticities at the respective equilibrium extensive margins. Thus, higher values of $\varepsilon_{L}$ and $\varepsilon_{H}$ denote a relative high comparative advantage of medium-skilled workers at the respective task margin, which in turn implies a low substitutability. As pointed out by Acemoglu and Autor (2011), this indicates an additional source of substitution effect, i.e. the substitutability of skills across tasks.

A corollary follows from Lemma 3.1

Corollary 1. For all $\frac{w_{L}}{A_{L}}\left(\beta_{L}(\tilde{I})\right)^{-1}=\frac{w_{H}}{A_{H}} \beta_{H}(\tilde{I})>\frac{w_{M}}{A_{M}}>\max \left\{\frac{w_{L}}{A_{L}} \beta_{L}(0)^{-1}, \frac{w_{H}}{A_{H}} \beta_{H}(1)\right\}$, it follows that $0<I_{L}<\tilde{I}<I_{H}<1$. 
This is a necessary and sufficient condition permitting the existence of all three skill groups in the economy. The lower boundary indicates that low-skilled workers are the most efficient ones at the least skill-intensive task $i=0$ and high-skilled workers are the most efficient ones at the most skill-intensive task $i=1$. In addition, the upper boundary ensures that medium-skilled workers have comparative advantages in the middle range of the task distribution. For example, if $\frac{w_{L}}{A_{L}} \beta_{L}(\tilde{I})^{-1}=\frac{w_{H}}{A_{H}} \beta_{H}(\tilde{I}) \leq \frac{w_{M}}{A_{M}}$, then employment consists of only low- and high-skilled workers. ${ }^{9}$ Medium-skilled workers have then no comparative advantage in performing any task relative to low- and high-skilled workers. To sum up, by Lemma 3.1 and Corollary 1, the domestic labor force is allocated over the unit interval as follows: low-skilled workers are employed in the interval $i \in\left[0, I_{L}\right]$, medium-skilled in $i \in\left(I_{L}, I_{H}\right)$, and high-skilled in $i \in\left[I_{H}, 1\right]$. We depict the equilibrium task allocation in Figure 3.1.

\subsubsection{Allocation of offshored tasks}

As discussed in the introduction, the empirical evidence shows that over recent decades there has been a strong tendency to reallocate domestic tasks abroad. This process particularly applies to jobs intensive in routine tasks and concentrated in the middle range of the skill distribution. This in turn may explain the recent trends in wage polarization in many advanced countries. Based on this pattern, Acemoglu and Autor (2011) investigate the distributional effect of offshoring by allowing a sub-range of domestic tasks, which were previously performed by medium-skilled workers, to be exogenously offshored.

However, this ad-hoc introduction of offshoring neglects to address another important aspect discussed in the literature: the offshoring-induced productivity effect. In order to address this effect, we need to account for changes at both extensive and intensive margins, while Acemoglu and Autor (2011) consider the infra-marginal effect of offshorin, jumping from zero offshoring to some positive offshoring scenarios. The motivation for our deviation is that the impact of offshoring on the cost structure will depend on the level of additionally-offshored tasks (extensive margin) as well as on the size of tasks that have already been offshored (intensive margin).

We augment the general task-based framework of Acemoglu and Autor (2011) by allowing domestic firms to choose endogenously the cost-optimal range of tasks to be offshored. As we discuss below, this in turn requires a non-monotonic comparative advantage schedule between domestic medium-skilled and offshore workers. More precisely, the relative taskproductivity schedule becomes a U- (or V-)shaped relationship. Moreover, as we discuss below, any scenario of offshoring can be described by this U-shaped functional form. Formally, we impose the following assumption

\section{Assumption 2. (Offshoring Task Productivity)}

Defining the job-task productivity between medium-skilled and offshore workers by $\zeta(i)=$ $\frac{a_{M}(i)}{a_{O}(i)}$, there exists a threshold $\check{I}$ such that for all $i \in[0, \check{I}), \zeta(i)$ is (strict) monotonically decreasing, and for all $i \in(\breve{I}, 1], \zeta(i)$ is (strict) monotonically increasing.

\footnotetext{
${ }^{9}$ Notice that at strict equality the employer is indifferent between all three skill groups at margin $\tilde{I}$.
} 
Let the offshoring wage rate be given by $w_{O}$. Then, a domestic firm engaged in offshoring chooses the optimal amount of offshore workers, given the wage rate $w_{O}$, according to the following unit cost of producing task $i$ abroad: $c_{O}(i)=\tau \frac{w_{O}}{A_{O} a_{O}(i)}$. The term $\frac{\tau w_{O}}{A_{O}}$ is exogenous to the domestic firm and comprises the aforementioned three channels of globalization process, a feature that is missing in Acemoglu and Autor (2011). For example, a decline in $w_{O}$ is associated with skill accumulation abroad, indicating the endowment effect, an increase in $A_{O}$ denotes advances in foreign technology, the technology effect, and a decline in $\tau$ indicates lower transportation barriers, the trade cost effect. Summarizing all these exogenous channels by $\omega \equiv \frac{A_{O}}{\tau w_{O}}$, lower offshoring friction is now captured by $\mathrm{d} \omega>0$. Although each channel generates a similar qualitative effect, accounting explicitly for each of them has the following advantages. First, it allows us to address the implication of various forms of globalization, e.g. North-South vs. EastWest type of trade and offshoring activities. Second, it enables us to provide clear policy guidance regarding how the source country of offshoring could react to the increasing global competition on domestic jobs, e.g. if lower offshoring friction is due to $\mathrm{d} w_{O}<0$, then one possible response could be to increase the trading costs $\mathrm{d} \tau>0$; if it is due to $\mathrm{d} A_{O}>0$, then the source economy could possibly invest more in $\mathrm{R} \& \mathrm{D}$ to increase its technology frontier on those job tasks (i.e. $\mathrm{d} A_{M}>0$ ). The optimal task allocation between foreign and domestic medium-skilled workers is summarized as follows.

Lemma 3.2. By Assumption 2, there exist two task margins where a firm is indifferent between the domestic medium-skilled and offshore workers, i.e.

$$
\begin{gathered}
\frac{\zeta\left(I_{1}\right)}{\omega}=\frac{w_{M}}{A_{M}} \\
\frac{\zeta\left(I_{2}\right)}{\omega}=\frac{w_{M}}{A_{M}} .
\end{gathered}
$$

Proof. See Appendix 3.B.2.

Thus, by Lemma 3.2 the effect of offshoring is captured by the two endogenous offshoring boundaries, $I_{1}$ and $I_{2}$. They capture both the extensive margin, i.e. the offshorability of the marginal domestic task, as well as the intensive margin, i.e. the amount of tasks that have been already offshored. However, it is useful and sufficient to consider changes to the length of offshoring interval, i.e.

$$
I_{O}=I_{2}-I_{1},
$$

which accounts implicitly for two conditions, (3.5) and (3.6). This has analytical convenience. Let the semi-elasticities at the two offshoring margins be constant and defined as $\varepsilon_{1}=-\frac{\partial \ln \zeta_{1}\left(I_{1}\right)}{\partial I_{1}}>0$ and $\varepsilon_{2}=\frac{\partial \ln \zeta_{2}\left(I_{2}\right)}{\partial I_{2}}>0$. Then, by a simple positive monotone transformation, we obtain

Lemma 3.3. If Lemma 3.2 holds, then the two no-arbitrage conditions determining the task reallocation between medium-skilled and offshore workers can be expressed as one monotonically increasing function in the length of the offshorable task interval

$$
\frac{w_{M}}{A_{M}}=\frac{\beta_{O}\left(I_{O}\right)}{\omega},
$$


with $\beta_{O}\left(I_{O}\right)=\exp \left[\mu I_{O}\right]$ and $\mu=\frac{\varepsilon_{2} \varepsilon_{1}}{\varepsilon_{2}+\varepsilon_{1}}>0$.

Proof. See Appendix 3.B.3.

From Lemma 3.2 and Lemma 3.3 it follows

Corollary 2. For all $\omega$ in the interval $\left.\left.\frac{A_{M}}{w_{M}} \beta_{O}(0)<\omega<\left\{\frac{A_{M}}{w_{M}} \zeta\left(I_{L}\right)\right), \frac{A_{M}}{w_{M}} \zeta\left(I_{H}\right)\right)\right\}$, and $I_{L}<\left.\breve{I}\right|_{\frac{\partial \ln \zeta(\cdot)}{\partial i}=0}<I_{H}$, it follows that $I_{O}>0$ and $I_{O} \in\left(I_{L}, I_{H}\right)$.

Thus Corollary 2 ensures that offshoring activities are permitted, but limited to a subrange of medium-skilled job tasks. Put differently, if $\omega$ is sufficiently low such that $\omega=\frac{A_{M}}{w_{M}} \zeta\left(I_{L}\right)$ (or $\left.\omega=\frac{A_{M}}{w_{M}} \zeta\left(I_{H}\right)\right)$, then there is direct competition between foreign and domestic low-skilled (or high-skilled) workers. ${ }^{10}$ Thus, for all four types of workers to exist in the economy, we assume that both Corollaries 1 and 2 hold. These conditions, as well as the equilibrium task allocations, are sketched in Figure 3.1.

Figure 3.1: Equilibrium task allocation

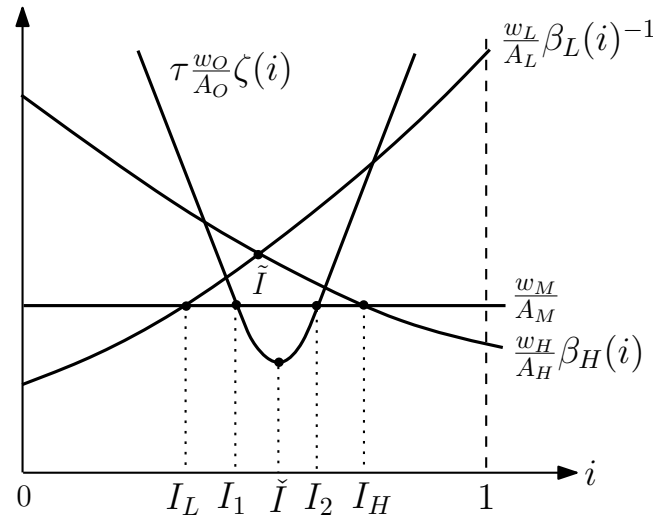

Summarizing the results above, the task production function emerges as

$$
t(i)= \begin{cases}A_{L} a_{L}(i) l_{L}(i) & \text { for all } i \in\left[0, I_{L}\right] \\ A_{M} a_{M}(i) l_{M}(i) & \text { for all } i \in\left(I_{L}, I_{H}\right) \backslash I_{O} \\ A_{O} a_{O}(i) l_{O}(i) / \tau & \text { for all } i \in I_{O} \\ A_{H} a_{H}(i) l_{H}(i) & \text { for all } i \in\left[I_{H}, 1\right] .\end{cases}
$$

Equation (3.8), therefore, reveals the new feature of our model. First, the range of offshorable tasks is determined endogenously. Second, the reallocation of domestic medium-skilled tasks and the size of the expansion of offshorable tasks is crucially determined by the relative productivity schedules in the neighborhood of $I_{1}$ and $I_{2}$, i.e. by the parameters $\varepsilon_{1}$ and $\varepsilon_{2}$. Below, we elaborate analytically on these features.

\footnotetext{
${ }^{10}$ Notice that such an outcome - as pointed out by Blinder (2009a,c) - might not be unrealistic. However, the polarization effect would become ambiguous due to the direct competition of offshore workers with low-skilled (or high-skilled) workers next to medium-skilled workers.
} 


\subsection{Production technology and labor demand}

The economy produces a final (consumption) good under perfect competition. The output is generated by using a labor composite, E, according to the following Cobb-Douglas technology function

$$
Y=B E^{1-\alpha}, \quad \alpha \in(0,1),
$$

where $B$ is a positive parameter that may contain exogenous variables such as total factor productivity (TFP) and physical capital, and $\alpha$ denotes the standard share of physical capital. Thus the production function (5.1) has diminishing returns in labor input. ${ }^{11}$ We consider $Y$ as the numeraire, i.e. $P_{Y}=1$. The labor composite input is, in turn, described by differentiated tasks, over a unit interval, according to the following Cobb-Douglas technology: ${ }^{12}$

$$
E=\exp \left(\int_{0}^{1} \ln (t(i)) \mathrm{d} i\right) .
$$

This specification allows us to deliver new insights regarding the offshoring-induced costefficiency effect.

\subsubsection{Optimal labor demand}

The firm's optimization problem is as follows. Taking the output price as given, the representative firm maximizes its profit by choosing the optimal amount of the labor composite factor. Formally, this is given by

$$
\max _{E} \pi=Y-P_{E} E \text {, s.t. (5.1). }
$$

The solution yields the optimal total employment

$$
E=P_{E}^{-\frac{1}{\alpha}} \mathcal{B}
$$

where $\mathcal{B}=((1-\alpha) B)^{1 / \alpha}$ and $P_{E}$ denotes the price index of the labor composite, which will be defined below. The optimal amount of labor input per job task is determined by means of cost minimization. Formally, we write the optimal programming of the firm as follows:

$$
\begin{aligned}
C_{E}^{*}\left(w_{L}, w_{M}, w_{H}, w_{O}\right) \equiv & \min _{l_{L}(i), l_{M}(i), l_{H}(i), l_{O}(i)}\left[w_{L} \int_{0}^{I_{L}} l_{L}(i) \mathrm{d} i+w_{M} \int_{i \in \mathcal{S}_{\mathcal{M}}} l_{M}(i) \mathrm{d} i\right. \\
& \left.+w_{O} \int_{i \in I_{O}} l_{O}(i) \mathrm{d} i+w_{H} \int_{I_{H}}^{1} l_{H}(i) \mathrm{d} i\right] \quad \text { s.t. (3.8), (3.10), }
\end{aligned}
$$

where the corresponding set of domestic medium-skill-intensive job tasks is denoted by $\mathcal{S}_{\mathcal{M}}=\left(I_{H}-I_{L}-I_{O}\right)$.

\footnotetext{
${ }^{11}$ Notice that when $B=1$ and $\alpha=0$, equation (5.1) reduces to the one used by Acemoglu and Autor (2011).

${ }^{12}$ Grossman and Rossi-Hansberg (2008) assume perfect complementarity, i.e. a Leontief production function. Ottaviano et al. (2013) assume a more general functional form by using a CES production technology.
} 
The corresponding minimized cost function of the labor composite is given by:

$$
\begin{aligned}
C_{E}^{*}(\cdot)= & \exp \left[\int_{0}^{I_{L}} \ln \left(\frac{w_{L}}{A_{L} a_{L}(i)}\right) \mathrm{d} i+\int_{i \in \mathcal{S}_{\mathcal{M}}} \ln \left(\frac{w_{M}}{A_{M} a_{M}(i)}\right) \mathrm{d} i\right. \\
& \left.+\int_{i \in I_{O}} \ln \left(\frac{\tau w_{O}}{A_{O} a_{O}(i)}\right) \mathrm{d} i+\int_{I_{H}}^{1} \ln \left(\frac{w_{H}}{A_{H} a_{H}(i)}\right) \mathrm{d} i\right] E .
\end{aligned}
$$

Then, by Shepard's lemma, we obtain the optimal labor demand per task: ${ }^{13}$

$$
\begin{aligned}
& \frac{\partial C_{E}^{*}(\cdot)}{\partial w_{L}}=\left(\frac{w_{L}}{P_{E}}\right)^{-1} E=l_{L} \\
& \frac{\partial C_{E}^{*}(\cdot)}{\partial w_{M}}=\left(\frac{w_{M}}{P_{E}}\right)^{-1} E=l_{M} \\
& \frac{\partial C_{E}^{*}(\cdot)}{\partial w_{H}}=\left(\frac{w_{H}}{P_{E}}\right)^{-1} E=l_{H} \\
& \frac{\partial C_{E}^{*}(\cdot)}{\partial w_{O}}=\left(\frac{w_{O}}{P_{E}}\right)^{-1} E=l_{O},
\end{aligned}
$$

where we used the fact that in perfect competitive equilibrium price equals marginal costs, i.e.

$$
\begin{aligned}
P_{E}= & \exp \left[\int_{0}^{I_{L}} \ln \left(\frac{w_{L}}{A_{L} a_{L}(i)}\right) \mathrm{d} i+\int_{i \in \mathcal{S}_{\mathcal{M}}} \ln \left(\frac{w_{M}}{A_{M} a_{M}(i)}\right) \mathrm{d} i\right. \\
& \left.+\int_{i \in I_{O}} \ln \left(\frac{\tau w_{O}}{A_{O} a_{O}(i)}\right) \mathrm{d} i+\int_{I_{H}}^{1} \ln \left(\frac{w_{H}}{A_{H} a_{H}(i)}\right) \mathrm{d} i\right] .
\end{aligned}
$$

The price index contains all three exogenous variables leading to changes in the offshoring friction: foreign technology $\left(A_{O}\right)$, foreign wages $\left(w_{O}\right)$, offshoring trade cost $(\tau)$. Recall that $\omega=\frac{A_{O}}{w_{O} \tau}$, then it can be shown that the partial effect of easier offshoring $(\mathrm{d} \omega>0)$ causes the marginal (average) cost of labor to decrease. That is,

$$
\frac{\partial P_{E}}{\partial \omega}<0
$$

\footnotetext{
${ }^{13}$ In Appendix 3.A.1, we derive the minimized cost function $C^{*}(\cdot)$ and show that at the optimum the number of workers per task is constant across the respective range of tasks. This is particularly due to the Cobb-Douglas assumption of the production technology as well as the law of one price within each skill group. This implies that the marginal productivity within each skill-task group is constant, i.e. $l(i)=l, m(i)=m$, and so on. See Firpo et al. (2011) for a critical discussion regarding the assumption of law of one price.
} 


\subsubsection{Equilibrium marginal cost}

The impact of lower offshoring friction refers to what is called the offshoring-induced costefficiency improvement in the literature. ${ }^{14}$ However, the general equilibrium implications (i.e. taking into account changes in the task margins) reveal a countervailing effect due to an internal (domestic) reallocation of workers, which is generally ignored in the literature. To account for this, Eq. (3.15) needs further manipulation. Utilize the cut-off conditions (3.2) and (3.3) from Lemma 3.1 to substitute for $\frac{w_{L}}{A_{L}}$ and $\frac{w_{H}}{A_{H}}$, respectively. Analogously, utilize the no-arbitrage condition (3.7) from Lemma 3.3 to substitute for $\frac{\tau w_{O}}{A_{O}}$. Then, $P_{E}$ reduces to

$$
\begin{aligned}
P_{E}= & \frac{w_{M}}{A_{M}} \exp \left[\int_{0}^{I_{L}} \ln \left(\frac{\beta_{L}\left(I_{L}\right)}{a_{L}(i)}\right) \mathrm{d} i-\int_{i \in \mathcal{S}_{\mathcal{M}}} \ln \left(a_{M}(i)\right) \mathrm{d} i\right. \\
& \left.+\int_{i \in I_{O}} \ln \left(\frac{1}{\beta_{O}\left(I_{O}\right) a_{O}(i)}\right) \mathrm{d} i+\int_{I_{H}}^{1} \ln \left(\frac{1}{\beta_{H}\left(I_{H}\right) a_{H}(i)}\right) \mathrm{d} i\right] .
\end{aligned}
$$

Decompose the term $\int_{i \in \mathcal{S}_{\mathcal{M}}} \ln \left(a_{M}(i)\right) \mathrm{d} i$ to combine it with the terms $\ln a_{L}(i), \ln a_{O}(i)$, and $\ln a_{H}(i)$. Then, after some rearranging and manipulation, we obtain

$$
P_{E}=\frac{w_{M}}{A_{M}} \Omega\left(I_{L}, I_{H}, I_{O}\right)
$$

where $^{15}$

$$
\begin{aligned}
\Omega(\cdot)= & \exp \left[\left(\int_{0}^{I_{L}} \ln \left(\frac{\beta_{L}\left(I_{L}\right)}{\beta_{L}(i)}\right) \mathrm{d} i+\int_{I_{H}}^{1} \ln \left(\frac{\beta_{H}(i)}{\beta_{H}\left(I_{H}\right)}\right) \mathrm{d} i\right.\right. \\
& \left.\left.+\int_{i \in I_{O}} \ln \left(\frac{\zeta(i)}{\beta_{O}\left(I_{O}\right)}\right) \mathrm{d} i-\int_{0}^{1} \ln \left(a_{M}(i)\right) \mathrm{d} i\right)\right]
\end{aligned}
$$

denotes the generalized common part of the marginal task cost. Thus this common part $\Omega$ accounts for the general equilibrium effect due to changes in the offshorability of domestic tasks. Contrary to the original version of Grossman and Rossi-Hansberg (2008), where $\Omega$ is simply defined as a function of one offshoring margin, easier offshoring does not necessarily induce a cost-efficiency effect. As we elaborate below, it depends

\footnotetext{
${ }^{14}$ It is worth mentioning that this effect is omitted in Acemoglu and Autor (2011) as the price index $P_{E}$ is set to unity.

${ }^{15}$ More precisely, after substitution, the term within the square brackets becomes

$$
\begin{aligned}
& {\left[\int_{0}^{I_{L}} \ln \left(\frac{\beta_{L}\left(I_{L}\right)}{a_{L}(i)}\right) \mathrm{d} i+\int_{0}^{I_{L}} \ln \left(a_{M}(i)\right) \mathrm{d} i+\int_{I_{H}}^{1} \ln \left(a_{M}(i)\right) \mathrm{d} i+\int_{i \in I_{O}} \ln \left(a_{M}(i)\right) \mathrm{d}-\int_{0}^{1} \ln \left(a_{M}(i)\right) \mathrm{d} i\right.} \\
& \left.+\int_{i \in I_{O}} \ln \left(\frac{1}{\beta_{O}\left(I_{O}\right) a_{O}(i)}\right) \mathrm{d} i+\int_{I_{H}}^{1} \ln \left(\frac{1}{\beta_{H}\left(I_{H}\right) a_{H}(i)}\right) \mathrm{d} i\right] .
\end{aligned}
$$
}

As is readily seen, this expression can be reduced further. For example,

$$
\int_{0}^{I_{L}} \ln \left(a_{M}(i)\right) \mathrm{d} i-\int_{0}^{I_{L}} \ln \left(a_{L}(i)\right) \mathrm{d} i=\int_{0}^{I_{L}} \ln \left(\beta_{L}(i)\right) \mathrm{d} i,
$$

and so on. Following these steps, we obtain Eq. (3.17). 
on the interaction between internal reallocation, i.e. medium-skilled workers to low- and high-skilled job tasks, and external reallocation, i.e. moving domestic jobs abroad.

\subsection{General equilibrium solution}

The general equilibrium closed solution to the equilibrium task margins is characterized by the cut-off conditions (3.2), (3.3) and (3.7) derived respectively in Lemma 3.1 and Lemma 3.3 , and the optimal labor demand functions, (3.14a)-(3.14d). From these conditions, we obtain a system of three equations determining simultaneously the implicit solution to the task margins, as we elaborate in this section.

From the optimal task-skill allocation (3.8) we obtain the labor-market-clearing condition

$$
\int_{0}^{I_{L}} l_{L}(i) \mathrm{d} i=N_{L}, \int_{i \in \mathcal{S}_{\mathcal{M}}} l_{M}(i) \mathrm{d} i=N_{M}, \int_{I_{H}}^{1} l_{H}(i) \mathrm{d} i=N_{H}, \int_{i \in I_{O}} l_{O}(i) \mathrm{d} i=n_{O},
$$

where $N_{k}, k \in\{L, M, H\}$ denotes the total (exogenously given) mass of domestic labor supply by skill and $n_{O}$ indicates the total (endogenous) mass of offshore employment. The labor-market-clearing condition (3.18) can be solved for $l_{L}, l_{M}, l_{H}$ and $l_{O}$. Utilizing the resulting expressions, respectively, in the demand functions (3.14a)-(3.14d) and solving for relative medium-skilled wages, we obtain

$$
\begin{aligned}
\frac{w_{L}}{w_{M}} & =\frac{N_{M}}{N_{L}} \frac{I_{L}}{I_{H}-I_{L}-I_{O}} \\
\frac{w_{M}}{w_{O}} & =\frac{n_{O}}{N_{M}} \frac{I_{H}-I_{L}-I_{O}}{I_{O}} \\
\frac{w_{M}}{w_{H}} & =\frac{N_{H}}{N_{M}} \frac{I_{H}-I_{L}-I_{O}}{1-I_{H}} .
\end{aligned}
$$

It is readily seen from Eqs. (3.19a), (3.19b) and (3.19c) that, ceteris paribus, the relative (inverse) demand functions are decreasing in the labor supply and increasing in the respective equilibrium task margins. Note, however, that Eq. (3.19b) contains on the right-hand side an additional endogenous variable, $n_{O}$, which is defined by the FOC (3.14d). Since the offshoring wage rate, $w_{O}$, is exogenously given for the domestic firm, any changes in the interval of offshorable tasks $I_{O}$ will affect $n_{O}$. To adjust for this effect, substitute for $n_{O}$ from the optimal demand equation (3.14d) and condition (3.18) to obtain

$$
\frac{n_{O}}{I_{O}}=\frac{P_{E} E}{w_{O}} .
$$

Furthermore, from (3.11) we get $P_{E} E=P_{E}^{-\frac{1-\alpha}{\alpha}} \mathcal{B}$. Substituting it back into $\left(3.14 \mathrm{~d}^{\prime}\right)$ yields

$$
\frac{n_{O}}{I_{O}}=\frac{P_{E}^{-\frac{1-\alpha}{\alpha}} \mathcal{B}}{w_{O}} .
$$

However, the price index is a function of task margins, thus endogenous too. To account for this, utilize the cut-off conditions (3.7) from Lemma 3.3 for $\frac{w_{M}}{A_{M}}$ in (3.16), and rearrange 
to obtain

$$
P_{E}=\frac{\beta_{O}\left(I_{O}\right)}{\omega} \Omega\left(I_{L}, I_{H}, I_{O}\right) .
$$

Substituting this for $P_{E}$ in $\left(3.14 \mathrm{~d}^{\prime \prime}\right)$, and combining the outcome with $(3.19 \mathrm{~b})$, we obtain the relative demand function

$$
\frac{w_{M}}{w_{O}}=\frac{\mathcal{B}}{\left(\frac{\beta_{O}\left(I_{O}\right)}{\omega} \Omega\left(I_{L}, I_{H}, I_{O}\right)\right)^{\frac{1-\alpha}{\alpha}} w_{O}} \frac{I_{H}-I_{L}-I_{O}}{N_{M}} .
$$

Combining now the Eqs. in (3.19a) and (3.19c), respectively, with the no-arbitrage conditions (3.2) and (3.3) as well as (3.20) with the no-arbitrage condition (3.7), after rearranging slightly, yields

$$
\begin{aligned}
\frac{N_{L} A_{L}}{N_{M} A_{M}} & =\frac{I_{L}}{\left(I_{H}-I_{L}-I_{O}\right) \beta_{L}\left(I_{L}\right)} \\
\frac{\mathcal{B}}{N_{M} A_{M}} \omega^{\frac{1}{\alpha}} & =\frac{\beta_{O}\left(I_{O}\right)^{\frac{1}{\alpha}} \Omega(\cdot)^{\frac{1-\alpha}{\alpha}}}{I_{H}-I_{L}-I_{O}} \\
\frac{N_{M} A_{M}}{N_{H} A_{H}} & =\frac{I_{H}-I_{L}-I_{O}}{\left(1-I_{H}\right) \beta_{H}\left(I_{H}\right)},
\end{aligned}
$$

where again $\omega \equiv \frac{A_{O}}{\tau w_{O}}$ captures exogenous changes in offshorability. We summarize the equilibrium characteristics in the following proposition.

Proposition 3.1 (Unique Equilibrium). By Lemmata 3.1, 3.2, and 3.3, together with the Corollaries 1 and 2, the system of equations (3.21a)-(3.21c) determines the unique equilibrium values for all endogenous task margins $\left\{I_{L}, I_{H}, I_{O}\right\}$ as a function of the exogenous variables and parameters.

Proof. A rigorous formal discussion is provided in the supplementary mathematical Appendix 3.B.4.

Notice that the left-hand side of the Eqs. in (3.21) consists only of exogenous variables and parameters of the model. The right-hand sides denote the medium-skilled labor demand relative to other type of workers at the equilibrium set of tasks. For example, in Eq. (3.21a) the right-hand side can be seen as the relative demand for low-skill-intensive tasks, in (3.21c) the relative demand for high-skill-intensive tasks is denoted, and so on. Therefore the $3 \times 3$ system of equations in (3.21) determines the general equilibrium closed solution of the endogenous task margins.

\subsection{Comparative statics}

Utilizing the system (3.21a)-(3.21c), we compute in this section the general equilibrium effects of easier offshoring on the endogenous equilibrium margins. Particularly, as mentioned earlier, easier offshoring is associated with $\mathrm{d} \omega>0$ induced either by i) an endowment effect, lower foreign wage costs $\left(\mathrm{d} w_{O}<0\right)$ due to accumulation of human capital abroad; ii) a technology effect, advances in technology $\left(\mathrm{d} A_{O}>0\right)$, e.g. owing 
to utilization of more advanced machines abroad; and/or iii) a trade cost effect, lower offshoring $\operatorname{cost}(\mathrm{d} \tau<0)$, e.g. because of abolition of transportation barriers.

Therefore, next to trade cost, we explicitly account for two additional channels of increasing globalization of the production process. This feature is missing from both the original framework of Grossman and Rossi-Hansberg (2008) and the generalized one of Acemoglu and Autor (2011).

\subsubsection{Increasing offshorability and task reallocation}

Taking logs in the equations derived in (3.21) and rearranging, we obtain

$$
\begin{gathered}
-\ln \left(\frac{N_{L} A_{L}}{N_{M} A_{M}}\right)+\ln I_{L}-\ln \left(I_{H}-I_{L}-I_{O}\right)-\ln \beta_{L}\left(I_{L}\right)=0 \\
-\ln \left(\frac{\mathcal{B}}{A_{M} N_{M}}\right)-\ln \left(I_{H}-I_{L}-I_{O}\right)+\frac{1}{\alpha} \ln \beta_{O}\left(I_{O}\right)+\frac{1-\alpha}{\alpha} \ln \Omega(\cdot)=\frac{1}{\alpha} \ln \omega \\
-\ln \left(\frac{N_{M} A_{M}}{N_{H} A_{H}}\right)+\ln \left(I_{H}-I_{L}-I_{O}\right)-\ln \beta_{H}\left(I_{H}\right)-\ln \left(1-I_{H}\right)=0 .
\end{gathered}
$$

Now we can compute the impact of easier offshoring on the task margins. We summarize the main results in the following proposition.

Proposition 3.2 (Easier Offshoring of Medium-Skilled Tasks \& Changes in Task Margins). Qualitatively, easier offshoring ( $\mathrm{d} \omega>0)$, due to advances in offshoring-biased technology $\left(\mathrm{d} A_{O}>0\right)$, or lower offshoring cost $(\mathrm{d} \tau<0)$, or a decline in foreign wage costs $\left(\mathrm{d} w_{O}<0\right)$, induces an expansion of the offshorable task range and a contraction of low- and high-skill-intensive tasks ranges

$$
\frac{\mathrm{d} I_{L}}{\mathrm{~d} \omega}<0, \quad \frac{\mathrm{d} I_{O}}{\mathrm{~d} \omega}>0, \quad \frac{\mathrm{d} I_{H}}{\mathrm{~d} \omega}>0, \quad \text { and } \quad\left|\frac{\mathrm{d} I_{O}}{\mathrm{~d} \omega}\right|>\left|\frac{\mathrm{d} I_{H}}{\mathrm{~d} \omega}\right|+\left|\frac{\mathrm{d} I_{L}}{\mathrm{~d} \omega}\right| .
$$

The asymmetry in the domestic reallocation of medium-skilled workers is determined by the comparative advantage schedules

$$
\left|\frac{\mathrm{d} \ln I_{L}}{\mathrm{~d} \omega}\right| \lesseqgtr\left|\frac{\mathrm{d} \ln I_{H}}{\mathrm{~d} \omega}\right|, \Rightarrow\left|\frac{1+\varepsilon_{H}\left(1-{ }_{H}\right)}{1-I_{H}}\right| \lesseqgtr\left|\frac{1+\varepsilon_{L} I_{L}}{I_{L}}\right| .
$$

Proof. A full analytical proof is provided in Appendix 3.D.1.

The intuition can be explained in the following way. Easier offshoring increases the cost advantage for domestic firms to reallocate domestic job tasks abroad. This effect displaces the marginal medium-skilled workers performing job tasks in the neighborhood of $I_{O}$. The Walrasian nature of the labor market implies a downward wage adjustment of medium skilled workers. This in turn indicates that the no-arbitrage conditions in Lemma 3.1 are off equilibrium, which are reassured by a reallocation of displaced medium-skilled workers to low-skill- (i.e. lower $I_{L}$ ) and high-skill- (i.e. higher $I_{H}$ ) intensive job tasks. 
Thus, Proposition 3.2 highlights what Costinot and Vogel (2010) call a task upgrading at the high-skill-extensive margin, i.e. more medium-skilled workers produce former high-skilled tasks, and a task downgrading at the low-skill-extensive margin, i.e. more medium-skilled workers produce former low-skilled tasks. ${ }^{16}$ Again, the magnitude of the skill down- and upgrading is determined by substitutability of medium-skilled workers at the equilibrium task margins $I_{L}$ and $I_{H}$. Thus a relative low substitutability at the high-skill-intensive job tasks (higher values of $\varepsilon_{H}$ ) implies that medium-skilled workers are disproportionately allocated into low-skill-intensive job tasks. The empirical literature has highlighted a gradual increase in skill downgrading in many advanced countries. Particularly, medium-skilled workers are more likely to be downgraded into jobs (occupations) that require lower educational attainment (Brynin and Longhi, 2009). In the following section we deviate from the perfect competitive labor market assumption and investigate the potential crowding-out effect of low-skilled workers induced by offshoring.

\subsubsection{Offshoring and distributional effects}

As discussed in the introduction, in many advanced countries the recent evidence highlights a polarizing wage trend, i.e. a relative decline of medium-skilled wages compared to low- and high-skilled wages. This is in sharp contrast to wage developments in the past, where the burden of globalization regarding wage and employment cuts was mainly borne by low-skilled workers. Particularly, the evolution of earnings inequality followed a monotonic increase between the skill groups (for a neat survey of the literature, see Acemoglu and Autor, 2011). In this section, we address the distributional effect by conducting sequentially the comparative statics for different scenarios of global competition and show that offshoring generates income effects similar to factor-biased technology (cf. Acemoglu, 2002b). ${ }^{17}$ We summarize the main results in the following proposition.

Proposition 3.3 (Offshoring and Income Distribution). If offshoring activities are limited to low-skilled-intensive job tasks (indicating globalization trends in the past), then lower offshoring friction induces a distributional impact similar to skill-biased technology change, i.e. $\frac{\mathrm{d}\left(w_{L} / w_{M}\right)}{\mathrm{d} \omega}<0$ and $\frac{\mathrm{d}\left(w_{M} / w_{H}\right)}{\mathrm{d} \omega}<0$. If offshoring is permitted to mediumskilled job tasks (indicating recent globalization trends), then easier offshoring leads to a wage polarization effect, i.e. $\frac{\mathrm{d}\left(w_{L} / w_{M}\right)}{\mathrm{d} \omega}>0$ and $\frac{\mathrm{d}\left(w_{M} / w_{H}\right)}{\mathrm{d} \omega}<0$. If offshoring activities are limited to high-skill-intensive job tasks (indicating potential future globalization trends), then a marginal decline in offshoring friction lowers the wage gap between skill groups similar to unskilled-biased technology changes, i.e $\frac{\mathrm{d}\left(w_{L} / w_{M}\right)}{\mathrm{d} \omega}>0$ and $\frac{\mathrm{d}\left(w_{M} / w_{H}\right)}{\mathrm{d} \omega}>0$.

\footnotetext{
${ }^{16}$ Notice, however, that (easier) offshoring in our framework differs from Costinot and Vogel (2010, section VI.B.). Their results affirm a pervasive rise in wages of more skilled workers, i.e. an increase in inequality, induced by an implicit increase in the size of the relatively skill scarce foreign economy. In contrast, we follow up on the recent empirical findings on the offshoring-induced polarization effect, i.e. a decline in wages of medium-skilled workers relative to low- and high-skilled, and highlight different channels that may lead to easier offshoring.

${ }^{17}$ Notice that these different scenarios of globalization are motivated by wage trends at different time periods, discussed in the literature, and thus are used synonymously.
} 
Below we provide a graphical assessment of the distributional impact of each of the offshoring scenarios.

\section{Polarization Effect}

Given the results of the comparative statics for changes in the equilibrium task margins, we proceed now with the assessment of the distributional effect of offshoring of mediumskilled job tasks. In doing so, we need to recall the no-arbitrage conditions: Eq. (3.2): $\frac{w_{L}}{w_{M}}=\frac{A_{L}}{A_{M}} \beta_{L}\left(I_{L}\right)$, and Eq. (3.3): $\frac{w_{M}}{w_{H}}=\frac{A_{M}}{A_{H}} \beta_{H}\left(I_{H}\right)$; together with the labor demand functions for medium-skilled workers relative to low-skilled workers Eq. (3.19a): $D_{L M} \equiv$ $\frac{w_{L}}{w_{M}}=\frac{N_{M}}{N_{L}} \frac{I_{L}}{\dot{I}-I_{L}}$, and relative to high-skilled workers, Eq. (3.19c): $D_{M H} \equiv \frac{w_{M}}{w_{H}}=\frac{N_{H}}{N_{M}} \frac{I_{H}-\hat{I}}{1-I_{H}}$, where $\dot{I} \equiv I_{H}-I_{O}$ and $\hat{I} \equiv I_{L}+I_{O}$. Thus, for given $I_{H}$ and $I_{O}, D_{L M}$ is increasing in $I_{L}$, while higher (lower) values of $I_{O}\left(I_{L}\right)$ induce a leftward shift of $D_{L M}$. The relative labor demand curve between medium- and high-skilled workers $D_{M H}$ is increasing in $I_{H}$ for given values $I_{L}$ and $I_{O}$, while higher values of both $I_{O}$ and $I_{H}$ lead to a rightward shift of $D_{M H}$.

Figure 3.2: Offshoring medium-skilled job tasks and wage polarization

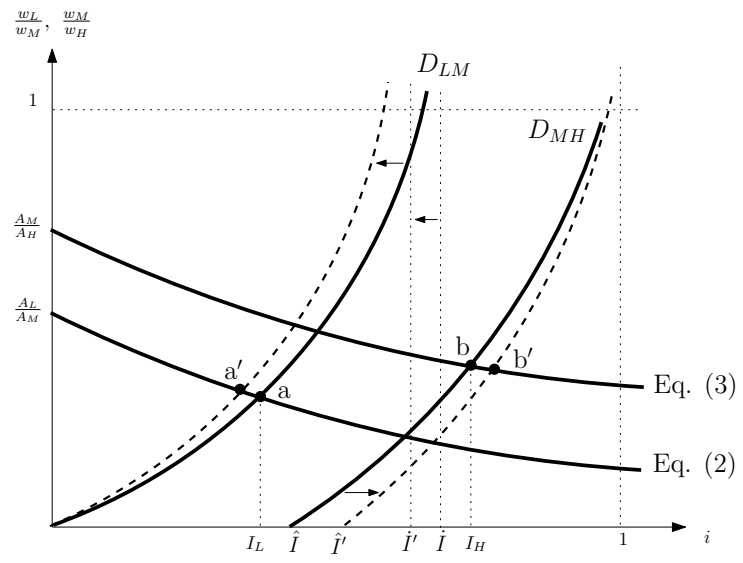

Moreover, it is readily seen that, for all exogenous shocks, but labor-biased technology $\left(A_{j}\right)$, changes in the relative medium-skilled wage compared to low- and high-skilled wages is a movement along the task productivity schedule, $\beta_{L}(\cdot)$ and $\beta_{H}(\cdot)$. To fix ideas, we illustrate the four conditions in Figure 3.2. Notice that the marginal decline in offshoring friction is captured by a decline in $\dot{I}$ and an increase in $\hat{I}$, implying a shift in the relative labor demand curves. From Proposition 3.2, the task margin $I_{L}$ will decline, while $I_{H}$ increases. This will lead to a slight backward shift in the relative labor demand curves. Eventually, the economy will reach the points $\mathrm{a}^{\prime}$ and $\mathrm{b}^{\prime}$, denoting the new equilibrium task margins. Notice that the internal reallocation of workers mitigates the wage polarization effect. The magnitude of this countervailing effect will have important implications for changes in the level of wages, which we elaborate next. 


\section{Skill-Biased Effect}

We now turn to the case where offshoring is permitted to low-skill-intensive job tasks. A formal discussion of this scenario is provided in Appendix 3.C. Notice again that any changes in relative wages between the skill groups due to exogenous changes in offshoring friction is a movement along the relative task productivity schedules, $\beta_{L}\left(I_{L}\right)$ and $\beta_{H}\left(I_{H}\right)$. The only difference compared to the previous case is that the relative labor demand conditions have changed. Now a (sub)range of low-skill-intensive job tasks is offshored, denoted by the equilibrium task margin $\tilde{I}_{O}$. Thus any changes in offshoring friction will directly shift the relative demand curve between low- and medium-skilled workers (denoted by $\tilde{D}_{L M}$ ) to the right. This induces changes in the task margin $I_{L}$, which in turn lead to a right-ward shift in the relative labor demand between medium- and high-skilled workers (denoted by $\tilde{D}_{M H}$ ). As a consequence, the task margin $I_{H}$ increases. We derive the relevant conditions in Appendix 3.C and Figure 3.3 provides a graphical illustration of them.

Figure 3.3: Offshoring low-skilled job tasks and skill-biased wage effect

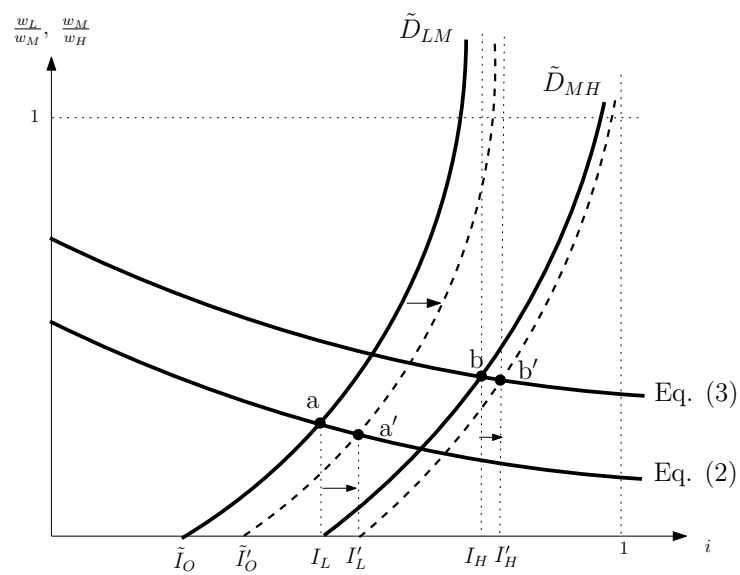

The intuitive mechanism behind the distributional effect of offshorability of low-skilled job tasks can be explained in the following way. Compared to medium-skilled workers, a decline in the offshoring friction $(\mathrm{d} \omega>0)$ increases the comparative advantages of offshore workers relative to low-skilled workers. Thus more low-skilled job tasks will be reallocated abroad that were previously performed by low-skilled workers. This implies higher values of the task margin $\tilde{I}_{O}$. Due to the Walrasian nature of the labor markets, the currently offshoring-induced unemployed low-skilled workers must be reemployed, leading to higher competition for available jobs and consequently to a decline of the low-skilled wage rate. This, in turn, raises the comparative advantages of low-skilled workers relative to medium-skilled workers.

Consequently, a proportion of medium-skill-intensive job tasks will be now allocated to low-skilled workers that were previously performed by medium-skilled workers (i.e. an increase in $I_{L}$ ). This effect is captured by a downward shift of the relative demand curve between low- and medium-skilled workers $\left(\tilde{D}_{L M}\right)$. Following similar logic, the relative demand curve between medium- and high-skilled workers will shift to the right. These 
adjustments are depicted in Figure 3.3, where the economy converges eventually to the new equilibrium points $\mathrm{a}^{\prime}$ and $\mathrm{b}^{\prime}$. As is readily seen, the skill premium, i.e. the relative wage between low- and medium-skilled as well as between medium- and high-skilled workers, increases monotonically. This outcome confirms the wage trends in the past due to international competition that were mainly borne by domestic unskilled workers and is in sharp contrast to recent polarizing wage trends in many advanced countries. Thus recent trends highlight the significant shift in the international competition for the domestic workforce.

\section{Unskilled-biased Effect}

As discussed in the introduction, many occupations that require a high level of skill for cognitive and complex tasks (think of computer programming, statistical analysis) may be at peril in future due to potential international competition (Bhagwati et al., 2004; Blinder, 2009b). We provide a intuitive discussion of the distributional impact of this possible future scenario and illustrate graphically the adjustment mechanism.

In this scenario offshoring occurs at the higher end of skill-task distribution, such that a proportion of domestic high-skill-intensive job tasks is only allocated abroad, denoted by $\hat{I}_{O}$. As in the previous case, the two no-arbitrage conditions (3.2) and (3.3) derived in Lemma 3.1 still hold. Thus the adjustment due to marginal changes in the offshoring friction occurs along the relative labor demand functions, which is depicted now by $\hat{D}_{L M}$ and $\hat{D}_{M H}$. Intuitively, the mechanism works similarly to the previous cases. A lower offshoring friction leads to an expansion of offshore job tasks. The now displaced highskilled workers are induced to compete for a lower range of job tasks available in the labor market, leading to a decline in their wages.

Figure 3.4: Offshoring high-skilled job-tasks and unskill-biased wage effects

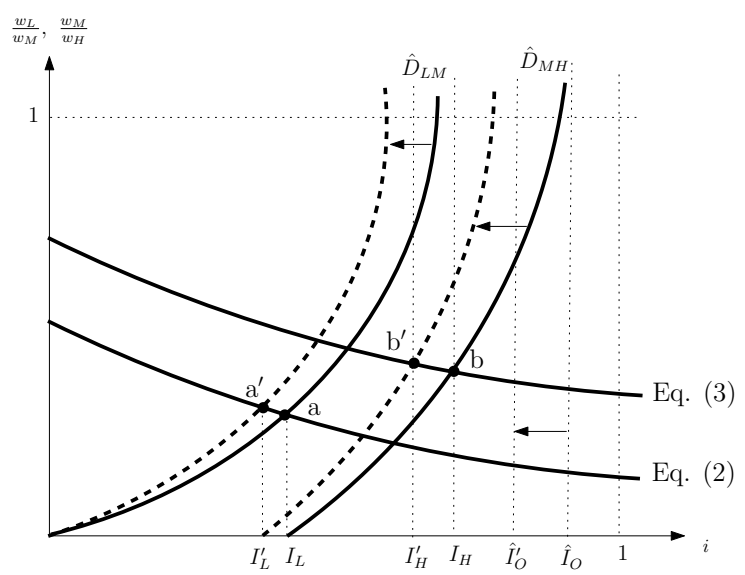

From the no-arbitrage condition (3.3) it follows that $I_{H}$ declines. This is illustrated in Figure 3.4 by a leftward shift in the relative labor demand curve between high- and medium-skilled workers $\hat{D}_{M H}$. Consequently the competition between medium-skilled workers increases, which pushes their wages down. From no-arbitrage condition (3.2) 
it can be verified that $I_{L}$ declines. This is depicted by a leftward shift of the relative demand curve $\hat{D}_{M H}$ in Figure 3.4. The labor market converges eventually to the new equilibrium points $\mathrm{a}^{\prime}$ and $\mathrm{b}^{\prime}$, which indicates an increase of relative wages of workers with an inferior skill level. In the next section, we turn back to the polarization case and derive the condition under which the real wage of all domestic workers increases due to the cost-efficiency effect.

\subsubsection{Offshoring-induced cost-efficiency effect}

As argued earlier, offshoring may generate a cost-efficiency effect similar to advances of technology. The rationale for this is that domestic firms engaged in offshoring benefit from easier offshoring at the extensive margin: more tasks can be moved abroad, as well as at the intensive margin: tasks that have already been offshored now become cheaper to import - a feature that is omitted in Acemoglu and Autor (2011). Therefore domestic firms experience a reduction in their average production cost, which in turn may lead to beneficial outcomes for the domestic workers.

As will become evident below, the offshoring-induced cost effects depend now on the interaction between the external reallocation and the internal, domestic reallocation of tasks. These two forces will affect the cost of composite labor $P_{E}$, which in turn might benefit all workers, even those who have been displaced directly. This new channel is in contrast to the task-based approaches discussed by Grossman and Rossi-Hansberg (2008), where every type of domestic worker is performing a distinct range of tasks and offshoring is characterized by one extensive margin, splitting task produced at home and abroad along this range. It also differs from the approach by Acemoglu and Autor (2011), where the cost index of composite labor $\left(P_{E}\right)$ is held constant.

To derive the effects of easier offshoring on the domestic real wage, we combine the domestic labor demand functions, (3.14a), (3.14b) and (3.14c), with the labor-marketclearing condition (3.18) to obtain the inverse labor demand functions

$$
\begin{aligned}
w_{L} & =\frac{I_{L}}{N_{L}} P_{E} E \\
w_{M} & =\frac{I_{H}-I_{L}-I_{O}}{N_{M}} P_{E} E \\
w_{H} & =\frac{1-I_{H}}{N_{H}} P_{E} E .
\end{aligned}
$$

Interestingly, the inverse labor demand conditions, (3.23), (3.24) and (3.25), imply that the cost share of each skill group is now denoted by the endogenous equilibrium range of tasks performed by the respective skill group. Moreover, the endogenous cost shares indicate the additional source of substitutability between skill groups across the tasks. Thus this new property can be seen as a generalization of the standard Cobb-Douglas function. $^{18}$

\footnotetext{
${ }^{18}$ However, notice the difference compared to Acemoglu and Autor (2011), where the task margins denote the expenditure share of each type of tasks in terms of the value of total output.
} 
Next, utilizing the optimal demand condition for E, Eq. (3.11), yields

$$
\begin{aligned}
w_{L} & =\frac{I_{L}}{N_{L}} P_{E}^{-\frac{1-\alpha}{\alpha}} \mathcal{B} \\
w_{M} & =\frac{I_{H}-I_{L}-I_{O}}{N_{M}} P_{E}^{-\frac{1-\alpha}{\alpha}} \mathcal{B} \\
w_{H} & =\frac{1-I_{H}}{N_{H}} P_{E}^{-\frac{1-\alpha}{\alpha}} \mathcal{B} .
\end{aligned}
$$

Recall now the definition of $P_{E}$ from Eq. (3.16) and, together with the no-arbitrage conditions (3.2) and (3.3) defined in Lemma 3.1, we obtain, after some rearranging

$$
\begin{aligned}
w_{L} & =\left(\frac{I_{L}}{N_{L}}\right)^{\alpha}\left(\beta_{L}\left(I_{L}\right)^{-1} \Omega(\cdot)\right)^{-(1-\alpha)} A_{L}^{1-\alpha} \mathcal{B}^{\alpha} \\
w_{M} & =\left(\frac{I_{H}-I_{L}-I_{O}}{N_{M}}\right)^{\alpha}(\Omega(\cdot))^{-(1-\alpha)} A_{M}^{1-\alpha} \mathcal{B}^{\alpha} \\
w_{H} & =\left(\frac{1-I_{H}}{N_{H}}\right)^{\alpha}\left(\beta_{H}\left(I_{H}\right) \Omega(\cdot)\right)^{-(1-\alpha)} A_{H}^{1-\alpha} \mathcal{B}^{\alpha} .
\end{aligned}
$$

These equations denote the generalized optimal demand for the domestic workforce that accounts for all endogenous equilibrium task margins. It is immediately evident that, given the properties of the task productivity schedules, $\beta_{L}(\cdot)$ and $\beta_{H}(\cdot)$, a decline in $\Omega(\cdot)$ will induce a positive demand effect for labor in the economy, thus increasing the wage level of all skills. We denote this effect as the cost-efficiency effect. Computing changes in $\Omega(\cdot)$, we obtain (see Appendix 3.A.2 for the formal derivation)

$$
\frac{\mathrm{d} \ln \Omega(\cdot)}{\mathrm{d} \omega}=\underbrace{\left(\left(1-I_{H}\right) \varepsilon_{H} \frac{\mathrm{d} I_{H}}{\mathrm{~d} \omega}-\varepsilon_{L} I_{L} \frac{\mathrm{d} I_{L}}{\mathrm{~d} \omega}\right)}_{\mathcal{R}>0}-\underbrace{\left(I_{O} \mu \frac{\mathrm{d} I_{O}}{\mathrm{~d} \omega}\right)}_{\mathcal{E}>0} .
$$

Changes due to internal reallocation, i.e. relocating medium-skilled workers towards lowand high-skill-intensive jobs is denoted by $\mathcal{R}$ and changes due to the external reallocation, i.e. moving domestic medium-skill-intensive tasks abroad, is denoted by $\mathcal{E}$. Notice that by the results of the comparative statics in Proposition 3.2, both the internal $\mathcal{R}$ and the external $\mathcal{E}$ allocation effects are positive.

Therefore, for the marginal production cost of composite labor $(\Omega)$ to decrease in the course of easier offshoring, the necessary condition requires $\mathcal{E}>\mathcal{R}$. As shown in Appendix 3.A.2, for the following sufficient low substitutability between medium-skilled and offshore workers, the external reallocation effect will be dominating, i.e.

$$
\mu>\frac{1-\left(I_{H}-I_{L}\right)}{I_{O}\left(I_{H}-I_{L}-I_{O}\right)} .
$$

However, this condition is not sufficient to ensure an increase in the real wage. The sufficient condition can be derived by straightforwardly differentiating the demand 
functions, (3.26), (3.27) and (3.28), which yields

$$
\begin{aligned}
& \frac{\mathrm{d} \ln w_{L}}{\mathrm{~d} \omega}=\left(\frac{\alpha}{I_{L}}-(1-\alpha) \varepsilon_{L}\right) \frac{\mathrm{d} I_{L}}{\mathrm{~d} \omega}-(1-\alpha) \frac{\mathrm{d} \ln \Omega}{\mathrm{d} \omega} \\
& \frac{\mathrm{d} \ln w_{M}}{\mathrm{~d} \omega}=-\frac{\alpha \mathrm{d} I_{L}}{I_{H}-I_{L}-I_{O}}-\frac{\alpha \mathrm{d} I_{O}}{I_{H}-I_{L}-I_{O}}+\frac{\alpha \mathrm{d} I_{H}}{I_{H}-I_{L}-I_{O}}-(1-\alpha) \frac{\mathrm{d} \ln \Omega}{\mathrm{d} \omega} \\
& \frac{\mathrm{d} \ln w_{H}}{\mathrm{~d} \omega}=\left((1-\alpha) \varepsilon_{H}-\frac{\alpha}{1-I_{H}}\right) \frac{\mathrm{d} I_{H}}{\mathrm{~d} \omega}-(1-\alpha) \frac{\mathrm{d} \ln \Omega}{\mathrm{d} \omega} .
\end{aligned}
$$

Now using Eq.(3.29) to substitute for $\frac{\mathrm{d} \ln \Omega}{\mathrm{d} \omega}$ in (3.31b) and rearranging the terms, we obtain

$$
\begin{aligned}
\frac{\mathrm{d} \ln w_{L}}{\mathrm{~d} \omega} & =\left(\frac{\alpha}{I_{L}}-(1-\alpha) \varepsilon_{L}\right) \frac{\mathrm{d} I_{L}}{\mathrm{~d} \omega}-(1-\alpha) \frac{\mathrm{d} \ln \Omega}{\mathrm{d} \omega} \\
\frac{\mathrm{d} \ln w_{M}}{\mathrm{~d} \omega} & =\left((1-\alpha) I_{L} \varepsilon_{L}-\frac{\alpha}{I_{H}-I_{L}-I_{O}}\right) \frac{\mathrm{d} I_{L}}{\mathrm{~d} \omega}+\left((1-\alpha) I_{O} \mu-\frac{\alpha}{I_{H}-I_{L}-I_{O}}\right) \frac{\mathrm{d} I_{O}}{\mathrm{~d} \omega} \\
& +\left(\frac{\alpha}{I_{H}-I_{L}-I_{O}}-(1-\alpha)\left(1-I_{H}\right) \varepsilon_{H}\right) \frac{\mathrm{d} I_{H}}{\mathrm{~d} \omega} \\
\frac{\mathrm{d} \ln w_{H}}{\mathrm{~d} \omega} & =\left((1-\alpha) \varepsilon_{H}-\frac{\alpha}{1-I_{H}}\right) \frac{\mathrm{d} I_{H}}{\mathrm{~d} \omega}-(1-\alpha) \frac{\mathrm{d} \ln \Omega}{\mathrm{d} \omega}
\end{aligned}
$$

Notice that defining the sign of Eq. $\left(3.31 b^{\prime}\right)$ determines also the relationship between internal (i.e. $\mathcal{E}$ ) and external (i.e. $\mathcal{R}$ ) task reallocation. The converse is obviously not true. Therefore, in $\left(3.31 \mathrm{a}^{\prime}\right)$ and $\left(3.31 \mathrm{c}^{\prime}\right)$ we need to elaborate the sign of the first term on the right hand side. We summarize the results in the following proposition.

Proposition 3.4 (Offshoring-induced Cost-efficiency Effect). Given the impact of easier offshoring of medium-skilled tasks on the task reallocation derived in Proposition 3.2, an offshoring-induced cost-efficiency improvement raises the real wage of all domestic worker for the following jointly sufficient conditions:

$$
\begin{aligned}
& \text { 1. } \frac{\alpha}{1-\alpha} \frac{1}{I_{L}\left(I_{H}-I_{L}-I_{O}\right)}>\varepsilon_{L}>\frac{\alpha}{(1-\alpha) I_{L}} \\
& \text { 2. } \frac{\alpha}{1-\alpha} \frac{1}{\left(1-I_{H}\right)\left(I_{H}-I_{L}-I_{O}\right)}>\varepsilon_{H}>\frac{\alpha}{1-\alpha} \frac{1}{\left(1-I_{H}\right)} \\
& \text { 3. } \mu>\frac{\alpha}{1-\alpha} \frac{1}{I_{O}\left(I_{H}-I_{L}-I_{O}\right)} .
\end{aligned}
$$

Proof. The lower boundary in part 3, as well as the upper limits in parts 1 and 2, follow straightforwardly from $\left(3.31 b^{\prime}\right)$. The lower limits in parts 1 and 2 are, respectively, derived from $\left(3.31 \mathrm{a}^{\prime}\right)$ and $\left(3.31 \mathrm{c}^{\prime}\right)$.

The intuition behind the jointly sufficient conditions in Proposition 3.4 is the following. Higher values of $\varepsilon_{L}\left(\varepsilon_{H}\right)$ imply a higher comparative advantage of low-skilled (high-skilled) workers in the neighborhood of $I_{L}\left(I_{H}\right)$ relative to medium-skilled workers. Now recall Eq. (3.2') and Eq. (3.3') to see the implications. It is readily evident that the size of the parameters $\varepsilon_{L}$ and $\varepsilon_{H}$ importantly determines the magnitude of the change of the task margins $I_{L}$ and $I_{H}$ for a one percentage change in relative wages, respectively. This effect is, as put forward in Acemoglu and Autor (2011), the additional source of substitutability 
between skill groups across tasks, next to the elasticity of substitution of unity arising from the Cobb-Douglas functional form.

The important implication of this additional substitution effect of skills across tasks can be inferred from the following special case. Take the limit $\varepsilon_{L} \rightarrow 0$ (indicating perfect substitutability between low- and medium-skilled workers at $\left.I_{L}\right)$, then from Eq. $\left(3.31 \mathrm{a}^{\prime}\right)$ it can be easily inferred that the first term reduces to $\frac{\alpha}{I_{L}} \frac{\mathrm{d} I_{L}}{\mathrm{~d} \omega}$, which by Proposition 3.2 is unambiguously negative. This is the offshoring-induced labor supply effect as the displaced workers have to be reabsorbed by the labor market. Hence, Proposition 3.4 highlights that the cost-efficiency effect due to easier offshoring will raise the real wage of all skill groups as the overall labor demand increases more than offshoring-induced increase in labor supply if the comparative advantages of low-skilled and high-skilled workers are sufficiently preserved.

\subsection{Equilibrium unemployment}

So far we have assumed full employment and analyzed the pure distributional effect of offshoring. However, another concern raised in the public debate on offshoring is the displacement effect of workers, leading to unemployment. In this section, we generalize the framework by allowing for equilibrium unemployment. In doing so, we assume that only low-skilled workers face the risk of unemployment. Intuitively and in line with our discussion in the introduction, easier offshoring may indirectly displace low-skilled workers from the labor market due to increasing competition with the offshoring-induced unemployed medium-skilled workers. In the literature, this potential displacement effect is referred to as the crowding-out effect; see Muysken et al. (2015) for a discussion.

We assume two alternative types of labor market friction, without altering the structure of the model. An intuitive source of friction is a minimum wage regime, which is set above the market equilibrium wage rate. Consequently, a proportion of low-skilled workers ends up unemployed. Alternatively, frictions can arise when we allow for endogenous supply of low-skill labor services. In this case the low-skilled wage is set as a mark-up over the unemployment benefits, where the mark-up depends negatively on unemployment rate. While the former is the mirror image of the full-employment case, characterized by a perfect elastic labor supply curve, the latter allows for an elastic labor supply curve and thus includes the standard approach of labor supply.

\subsubsection{Minimum wage regime}

Let the institutional minimum wage be $\bar{W}$. We assume that the minimum wage is set sufficiently low such that it is still attractive for domestic firms to employ low-skilled workers, but is sufficiently high such that a proportion of low-skilled workers ends up unemployed. Let $u_{L}$ denote the low-skilled unemployment rate. Formally, we impose the following assumption on the minimum wage scheme. 
Assumption 3. (Minimum wage setting)

$$
w_{L}<\bar{W}<\frac{w_{M} A_{L}}{A_{M}} \beta_{L}(0)
$$

where $w_{L}$ and $w_{M}$ are the equilibrium values resulting from the model analyzed in the previous section.

Given the level of minimum wage, the representative firm will then reallocate the job tasks between low- and medium-skilled workers such that the no-arbitrage condition (3.2) holds again, though at a lower equilibrium threshold. Moreover, from the general equilibrium perspective, our analysis implies that all other task margins will readjust too. The intuition is the following. A higher minimum wage scheme increases the relative comparative advantage of medium-skilled workers compared to low-skilled workers. Thus, from condition (3.2), the task margin $I_{L}$ must decline. This in turn implies that the range of tasks allocated to medium-skilled workers will increase, and from relative mediumskill labor demand conditions (3.19a)-(3.19c), medium skill wages will increase too. Consequently, the range of tasks performed by high-skilled $\left(1-I_{H}\right)$ and offshore workers $I_{O}$ must increase to satisfy again the no-arbitrage conditions (3.3) and (3.7).

In addition, compared to the full-employment case, now only a fraction of low-skilled workers can be hired, i.e. $n_{L}=\left(1-u_{L}\right) N_{L}$, and the resource constraint becomes $l=\frac{n_{L}}{I_{L}}$. This implies that the adjustment channel in the low-skill labor market is through employment instead of wages. Thus the relative demand condition for lowskilled workers defined in (3.19a) has to account for the endogenous adjustment in lowskilled employment. In order to investigate the impact of offshoring on the low-skilled unemployment rate, it is necessary to examine first the impact of offshoring on task allocation under the minimum wage scheme. We proceed with the derivation of the new equilibrium conditions, while, for the sake of illustration, we use the same expression for the equilibrium task margins as in the frictionless labor market scenario.

Recall the first-order condition (3.14a), where now the marginal productivity of low-skilled workers equals the minimum wage scheme, and utilize the low-skilled labor constraint to obtain

$$
\frac{n_{L}}{I_{L}}=\frac{P_{E}^{-\frac{1-\alpha}{\alpha}} \mathcal{B}}{\bar{W}},
$$

which is equivalent to the full-employment case, except that now the level of employment, $n_{L}$, is endogenous while the level of wage is fixed. Next, notice that the no-arbitrage condition (3.2) is defined now as

$$
\frac{\bar{W}}{w_{M}}=\frac{A_{L}}{A_{M}} \beta_{L}\left(I_{L}\right)
$$

Using this observation together with Eq. (3.16), we obtain

$$
P_{E}=\frac{\bar{W}}{A_{L}} \beta_{L}\left(I_{L}\right)^{-1} \Omega(\cdot) .
$$

Substituting Eq. (3.34) for $P_{E}$ in Eq. (3.32), we obtain the generalized low-skilled labor 
demand equation under a minimum wage scheme

$$
\frac{n_{L}}{I_{L}}=\frac{\left(\frac{\bar{W}}{A_{L}} \beta_{L}\left(I_{L}\right)^{-1} \Omega(\cdot)\right)^{-\frac{1-\alpha}{\alpha}} \mathcal{B}}{\bar{W}} .
$$

Utilizing this expression in the relative demand equation (3.19a), together with the new no-arbitrage condition (3.33), we obtain the modified implicit condition that accounts for the minimum wage scheme and the endogenous low-skilled employment

$$
\frac{\mathcal{B}}{A_{M} N_{M}}\left(\frac{A_{L}}{\bar{W}}\right)^{1 / \alpha}=\frac{\beta_{L}\left(I_{L}\right)^{-1 / \alpha} \Omega(\cdot)^{\frac{1-\alpha}{\alpha}}}{I_{H}-I_{L}-I_{O}} .
$$

This is equivalent to Eq. (3.21a) in the frictionless labor market scenario. Utilizing Eq. (3.36) together with Eqs. (3.21b) and (3.21c), we obtain the modified implicit $3 \times 3$ system of equations for the endogenous task margins.

Taking logs and rearranging slightly yields

$$
\begin{aligned}
-\frac{1}{\alpha} \ln \left(\beta_{L}\left(I_{L}\right)\right)+\frac{1-\alpha}{\alpha} \ln \left(\Omega\left(I_{H}, I_{L}, I_{O}\right)\right)-\ln \left(I_{H}-I_{L}-I_{O}\right)-\ln \left(\frac{\mathcal{B}}{A_{M} N_{M}}\right)-\frac{1}{\alpha} \ln \left(\frac{A_{L}}{\bar{W}}\right) & =0 \\
\frac{1}{\alpha} \ln \left(\beta_{O}\left(I_{O}\right)\right)+\frac{1-\alpha}{\alpha} \ln \left(\Omega\left(I_{H}, I_{L}, I_{O}\right)\right)-\ln \left(I_{H}-I_{L}-I_{O}\right)-\ln \left(\frac{\mathcal{B}}{A_{M} N_{M}}\right)-\frac{1}{\alpha} \ln (\omega) & =0 \\
\ln \left(I_{H}-I_{L}-I_{O}\right)-\ln \left(1-I_{H}\right)-\ln \left(\beta_{H}\left(I_{H}\right)\right)-\ln \left(\frac{A_{M} N_{M}}{A_{H} N_{H}}\right) & =0 .
\end{aligned}
$$

By straightforward differentiation of the system (3.37), one can compute the impact of an increase in the minimum wage scheme as well as easier offshoring on the equilibrium task margins. We summarize the main results in the following proposition and refer readers to the Appendix 3.D.2 and 3.D.3 for a formal proof.

Proposition 3.5 (Minimum Wage, Offshoring Medium-skilled Tasks, and Task Margins). If offshoring medium-skilled tasks is permitted and the low-skill labor market is characterized by a minimum wage scheme, then a rise in the minimum wage scheme will lead to a contraction of low-skill-intensive jobs tasks, i.e. $\frac{\mathrm{d} I_{L}}{\mathrm{~d} W}<0$, and an expansion of high-skill-intensive and offshorable job tasks, i.e. $\frac{\mathrm{d} I_{H}}{\mathrm{~d} W}<0$ and $\frac{\mathrm{d} I_{O}}{\mathrm{~d} \bar{W}}>0$, respectively. Easier offshoring generates similar skill-task reallocation as in Proposition 3.2.

Given these results, we can now assess the impact of easier offshoring in the low-skilled unemployment rate. In doing so, recall the low-skilled labor demand condition (3.35). Rearranging and taking logs, we obtain

$$
\ln n_{L}=\ln \left(I_{L}\right)+\frac{1-\alpha}{\alpha} \ln \left(\beta_{L}\left(I_{L}\right)\right)-\frac{1-\alpha}{\alpha} \ln (\Omega(\cdot))-\frac{1}{\alpha} \ln \bar{W}+\frac{1-\alpha}{\alpha} \ln A_{L}+\ln \mathcal{B} .
$$

Now total differentiating w.r.t. offshoring friction $(\mathrm{d} \omega>0)$ yields

$$
\frac{\mathrm{d} \ln n_{L}}{\mathrm{~d} \omega}=\left(\frac{1}{I_{L}}-\frac{(1-\alpha)}{\alpha} \varepsilon_{L}\right) \frac{\mathrm{d} I_{L}}{\mathrm{~d} \omega}-\frac{(1-\alpha)}{\alpha} \frac{\mathrm{d} \ln \Omega}{\mathrm{d} \omega} .
$$

The following proposition summarizes the main results regarding the impact of offshoring on low-skilled unemployment rate. 
Proposition 3.6 (Minimum Wage, Offshoring Medium-Skilled Tasks, and Low-Skilled Unemployment). If a fraction of low-skilled workers is unemployed due to a minimum wage scheme, then easier offshoring of medium-skilled tasks will lead to a decline in the low-skilled unemployment rate if and only if Proposition 3.4 holds.

The intuition is similar to the one discussed in Proposition 3.4. The difference is that now, with a minimum wage scheme, the low-skill labor market adjustment occurs via employment.

\subsubsection{Endogenous labor supply}

A more general approach to addressing labor market frictions is to allow workers to supply endogenously labor services, implying an elastic labor supply curve. This feature has important implications for labor market outcomes. In doing so, we follow the standard approach in the literature and assume that the low-skilled wage is a markup on unemployment benefits that depends negatively on the unemployment rate. This mark-up can be explained in many ways, such as the standard individual leisure-work choice, wage bargaining (Layard et al., 2005), search and matching theory à la Pissarides (2000) and efficiency wages à la Shapiro and Stiglitz (1984). Imposing such a negative relationship between the mark-up and unemployment induces an elastic labor supply curve. This way, we provide a more general analysis of labor market effects of offshoring compared to the minimum wage case. ${ }^{19}$

Let the endogenous low-skilled wage curve be defined by

$$
w_{L}=f\left(u_{L}\right) b_{L},
$$

where $f\left(u_{L}\right)$ denotes the mark-up over unemployment benefits, $b_{L}$, and has the following properties: $f\left(u_{L}\right)>1$ and $\frac{\partial f\left(u_{L}\right)}{\partial u_{L}}<0$. Moreover, we define the elasticity of the wage curve in absolute value w.r.t. $u_{L}$ as $\tilde{\delta} \equiv-\frac{\mathrm{d} \ln f\left(u_{L}\right)}{\mathrm{d} \ln u_{L}}>0$.

In this case, both the low-skilled wage and employment will adjust to exogenous shocks. Thus, compared to full employment and minimum wage cases, the low-skilled labor demand functions (3.26) and (3.35) become

$$
w_{L}=\left(\frac{I_{L}}{\left(1-u_{L}\right) N_{L}}\right)^{\alpha}\left(\frac{\beta_{L}\left(I_{L}\right)}{\Omega(\cdot)}\right)^{1-\alpha}\left(A_{L} \mathcal{B}\right)^{\alpha},
$$

where we utilized $n_{L}=\left(1-u_{L}\right) N_{L}$. This implies that the relative demand between lowand medium-skilled workers in Eq. (3.21a) has to account for the endogenous changes in low-skilled employment. Formally, we write this condition by

$$
\frac{N_{L} A_{L}}{N_{M} A_{M}}=\frac{1}{1-u_{L}} \frac{I_{L}}{\left(I_{H}-I_{L}-I_{O}\right) \beta_{L}\left(I_{L}\right)} .
$$

\footnotetext{
${ }^{19} \mathrm{It}$ is worth mentioning the important implications of applying different equilibrium unemployment paradigms regarding the adjustment mechanism of the labor market to exogenous shocks. However, our objective is not to explain the efficiency of various adjustment mechanisms, and thus we deliberately leave this to future research. For an application of search-matching and efficiency wage theories to the original task-based approach of Grossman and Rossi-Hansberg (2008), see Kohler and Wrona (2011).
} 
The model is closed by the adjusted market-clearing condition in the low-skilled labor market, i.e. from Eqs. (3.39) and (3.38)

$$
f\left(u_{L}\right) b_{L}=\left(\frac{I_{L}}{\left(1-u_{L}\right) N_{L}}\right)^{\alpha}\left(\frac{\beta_{L}\left(I_{L}\right)}{\Omega(\cdot)}\right)^{1-\alpha} A_{L}^{1-\alpha} \mathcal{B}^{\alpha} .
$$

Thus the new system of equations consists of Eqs. (3.21b), (3.21c), (3.40) and (3.41). Taking logs and rearranging slightly, we obtain

$$
\begin{aligned}
\ln \left(\frac{A_{L} N_{L}}{A_{M} N_{M}}\right) & =-\ln \left(1-u_{L}\right)+\ln I_{L}-\ln \left(I_{H}-I_{L}-I_{O}\right)-\ln \beta_{L}\left(I_{L}\right) \\
\ln \left(\frac{\mathcal{B}}{A_{M} N_{M}}\right)+\frac{1}{\alpha} \ln \omega= & \frac{1}{\alpha} \ln \beta_{O}\left(I_{O}\right)+\frac{1-\alpha}{\alpha} \ln \Omega(\cdot)-\ln \left(I_{H}-I_{L}-I_{O}\right) \\
\ln \left(\frac{A_{M} N_{M}}{A_{H} N_{H}}\right)= & -\ln \left(1-I_{H}\right)+\ln \left(I_{H}-I_{L}-I_{O}\right)-\ln \beta_{H}\left(I_{H}\right) \\
\alpha \ln \left(\frac{\mathcal{B}}{N_{L}}\right)-\ln b_{L}+(1-\alpha) \ln A_{L}= & \alpha \ln \left(1-u_{L}\right)+\ln f\left(u_{L}\right)+(1-\alpha)\left(\ln \Omega(\cdot)-\ln \beta_{L}\left(I_{L}\right)\right) \\
& -\alpha \ln I_{L} .
\end{aligned}
$$

This is the generalized $4 \times 4$ implicit system of equations that accounts for endogenous supply of labor services. Notice also that the left-hand side consists of all exogenous variables, while the right-hand side accounts for the four endogenous variables $I_{L}, I_{O}, I_{H}$ and $u_{L}$.

Now the marginal impact of offshoring on task margins and the low-skilled unemployment rate can be computed by straightforward differentiation of (3.42) w.r.t. $\omega$ and the endogenous variables. We summarize the main results in the following proposition.

Proposition 3.7 (Offshoring Medium-skilled Tasks, Labor Supply, and Low-skilled Unemployment). If the low-skilled labor market is characterized by an endogenous wage curve, where a fraction $u_{L}$ of low-skilled workers are unemployed, then offshoring of medium-skill job tasks unambiguously reduces the low-skilled unemployment rate for sufficiently low substitutability between medium- and high-skilled workers at task margin $I_{H}$ and between medium- and low-skilled workers at task margin $I_{L}$, i.e.

$$
\varepsilon_{H}>\frac{\alpha}{(1-\alpha) I_{H}\left(1-I_{H}\right)}, \quad \varepsilon_{L}>\frac{1}{(1-\alpha) I_{L}\left(I_{H}-I_{L}\right)} .
$$

Moreover, by the sufficient conditions in Proposition 3.4, real wages of all skill groups rise.

Proof. See Appendix 3.D.4.

Thus Proposition 3.7 highlights again the importance of comparative advantages in performing job tasks, which determine the magnitude of spillover effects induced by offshoring. Recall the interpretation of the parameters $\varepsilon_{L}$ and $\varepsilon_{H}$, capturing the substitutability of skill groups at the respective equilibrium task margin. The spillover of medium-skilled workers induced by offshoring is dominated by an overall rise in total employment if both high-skilled and low-skilled workers have sufficiently high comparative 
advantages in performing tasks in the neighborhood of $I_{H}$ and $I_{L}$, respectively. Moreover, notice that the lower boundaries in Proposition 3.7 dominate those in Proposition 3.4, but they are not binding since all derived boundaries are not necessary conditions. ${ }^{20}$ The intuition behind the real wage effect is equivalent to the one provided in Proposition 3.4.

In addition, it is important to notice the difference from the minimum wage case, where now higher comparative advantages of low- and high-skilled workers are required due to endogenous labor supply. Intuitively, the low-skilled labor market will adjust to the spillover effect as follows. The bumping down of medium-skilled workers (lower $I_{L}$ ) will displace some of low-skilled workers, who were previously performing those tasks in the neighborhood of $I_{L}$. The increase in the unemployment rate will in turn lead to a downward wage adjustment due to a lower mark-up (captured by a flatter wage curve), mitigating the relative rise in comparative advantage of medium-skilled workers.

\subsection{Conclusion}

In this chapter we have analyzed the general equilibrium effects of easier offshoring on task allocation within the domestic economy as well as in the foreign economy. We augment the current literature analyzing the implications of increasing international competition visà-vis the domestic labor force with respect to the following features. First, by introducing endogenous offshoring, we have augmented the framework of Acemoglu and Autor (2011), where offshoring is exogenously given. Second, we allow for a heterogeneous labor market, characterized by low-, medium- and high-skilled workers, where the allocation of each skill group to job tasks is based on a Ricardian type of comparative advantages along a unit interval. In doing so, we augment the seminal task-based approach of Grossman and Rossi-Hansberg (2008), where each group of labor is producing a distinct, completely independent range of tasks. In this way, we are able to address two contradictory hypotheses regarding the distributional impact of offshoring for domestic workers: a wage polarization effect and a cost-efficiency effect. Finally, we allow for equilibrium unemployment of low-skilled workers. In doing so, we are able to capture important externalities in the labor market, such as the bumping down of medium-skilled workers inducing a crowding-out effect of low-skilled workers.

The general equilibrium analysis provides several new insights, on which the existing theoretical literature has been silent. First, we show that any scenario of offshoring domestic job tasks can be described by a U-shaped relative productivity schedule between domestic and offshore workers. This allows us to address different stages of globalization, where the burden in terms of wage and employment cuts was borne by different skill groups, e.g. the trends in the past affected mainly low-skilled workers, while recent trends show increasing competition for medium-skilled workers, and potential future developments towards high-skilled workers. Moreover, our analysis reveals that offshoring of low-skill-intensive job tasks generates distributional effects similar to skill-biased technology changes - consistent with observations in the past, see Acemoglu (2002b),

\footnotetext{
${ }^{20}$ As discussed in the supplementary mathematical Appendix 3.D.4, it can be verified that for sufficiently low substitutability between medium-skilled and offshore workers (i.e. high values of $\mu$ ), the low-skilled unemployment rate might still decline, even if Proposition 3.7 is violated.
} 
offshoring medium-skill-intensive domestic job tasks induces a wage polarization effect (reflecting recent trends), while offshoring of high-skill-intensive domestic job tasks generates an income distribution effect similar to unskilled-biased technology changes (Goldin and Katz, 2009).

Second, we derive clear conditions that characterize the offshoring-induced cost-efficiency effect. We show that the cost-efficiency effect induced by offshoring domestic job tasks, what we refer to as the external reallocation, is countervailed by an internal reallocation of tasks to domestic workers. More precisely, the internal reallocation refers to reallocation of offshoring-induced displaced medium-skilled workers to low-skill- and high-skill-intensive job tasks. The balance between the two forces depends importantly on the substitutability between medium- and low-skilled workers and between high- and medium-skilled workers at the respective equilibrium task margins. More precisely, for sufficient low substitutability, the internal reallocation effect is dominated by the external one. In this case, all domestic skill groups benefit in terms of higher real wages. The importance of this internal reallocation has been put forward in the recent empirical literature (cf. Baumgarten et al., 2013; Hummels et al., 2014).

Finally, we elaborate the implications of the bumping-down effect of medium-skilled workers induced by easier offshoring for the low-skilled unemployment rate. Our analysis reveals that if the substitutability between medium- and low-skilled workers at the equilibrium task margin is sufficiently low (i.e. low-skilled workers have sufficiently higher comparative advantages in performing the domestic job tasks), then the crowding-out effect is offset by the offshoring-induced cost-efficiency effect, boosting the low-skilled labor market. 


\section{Appendix}

\section{A Derivation of equilibrium conditions}

\section{A.1 Firm optimization problem}

\section{Optimal labor demand}

Utilizing (3.8) in (3.10), then the Lagrangian to the cost-minimizing problem (3.12) is read as follows:

$$
\begin{aligned}
\mathcal{L}= & {\left[w_{L} \int_{0}^{I_{L}} l_{L}(i) \mathrm{d} i+w_{M} \int_{i \in \mathcal{S}_{\mathcal{M}}} l_{M}(i) \mathrm{d} i+w_{O} \int_{i \in I_{O}} l_{O}(i) \mathrm{d} i+w_{H} \int_{I_{H}}^{1} l_{H}(i) \mathrm{d} i\right] } \\
& +\lambda\left[E-\exp \left[\int_{0}^{I_{L}} \ln \left(A_{L} a_{L}(i) l_{L}(i)\right) \mathrm{d} i+\int_{i \in \mathcal{S}_{\mathcal{M}}} \ln \left(A_{M} a_{M}(i) l_{M}(i)\right) \mathrm{d} i\right.\right. \\
& \left.\left.+\int_{i \in I_{O}} \ln \left(A_{O} a_{O}(i) l_{O}(i) / \tau\right) \mathrm{d} i+\int_{I_{H}}^{1} \ln \left(A_{H} a_{H}(i) l_{H}(i)\right) \mathrm{d} i\right]\right],
\end{aligned}
$$

where $\lambda$ is the Lagrangian multiplier. The first-order conditions w.r.t. $l_{k}(i), k=\{L, M, H, O\}$, and $E$ are, respectively, given:

$$
\begin{aligned}
\frac{\partial \mathcal{L}}{\partial l_{L}(i)} & =w_{L}-\lambda \frac{E}{l_{L}(i)}=0, \Rightarrow l_{L}(i)=l_{L}\left(i^{\prime}\right)=l_{L}, \forall i \in\left[0, I_{L}\right] \\
\frac{\partial \mathcal{L}}{\partial l_{M}(i)} & =w_{M}-\lambda \frac{E}{l_{M}(i)}=0, \Rightarrow l_{M}(i)=l_{M}\left(i^{\prime}\right)=l_{M}, \forall i \in \mathcal{S}_{\mathcal{M}} \\
\frac{\partial \mathcal{L}}{\partial l_{H}(i)} & =w_{H}-\lambda \frac{E}{l_{H}(i)}=0, \Rightarrow l_{H}(i)=l_{H}\left(i^{\prime}\right)=l_{H}, \forall i \in\left[I_{H}, 1\right] \\
\frac{\partial \mathcal{L}}{\partial l_{O}(i)} & =w_{O}-\lambda \frac{E}{l_{O}(i)}=0, \Rightarrow l_{O}(i)=l_{O}\left(i^{\prime}\right)=l_{O}, \forall i \in I_{O} .
\end{aligned}
$$

The first-order conditions (3.A.43)-(3.A.46) indicate that the marginal productivity of workers across the respective range of tasks is equal and thus the required number of workers per task does not vary across the respective range of tasks. Utilizing these conditions in the constraint (3.10) and rearranging, we obtain

$$
\begin{aligned}
\lambda= & \exp \left[\int_{0}^{I_{L}} \ln \left(\frac{w_{L}}{A_{L} a_{L}(i)}\right) \mathrm{d} i+\int_{i \in \mathcal{S}_{\mathcal{M}}} \ln \left(\frac{w_{M}}{A_{M} a_{M}(i)}\right) \mathrm{d} i\right. \\
& \left.+\int_{i \in I_{O}} \ln \left(\frac{\tau w_{O}}{A_{O} a_{O}(i)}\right) \mathrm{d} i+\int_{I_{H}}^{1} \ln \left(\frac{w_{H}}{A_{H} a_{H}(i)}\right) \mathrm{d} i\right] .
\end{aligned}
$$

By the envelope theorem, the marginal (average) cost of the labor composite is denoted by the shadow price, i.e. $\frac{\partial \mathcal{L}}{\partial E}=\lambda$. Thus, under perfect competition, the marginal cost equals the cost index of composite labor mentioned in the text, i.e. $P_{E}=\lambda$.

\section{A.2 Cost-efficiency effect}

Recall Eq. (3.17) and take logs to obtain

$$
\ln \Omega(\cdot)=\left[\left(\int_{0}^{I_{L}} \ln \left(\frac{\beta_{L}(i)}{\beta_{L}\left(I_{L}\right)}\right) \mathrm{d} i+\int_{I_{H}}^{1} \ln \left(\frac{\beta_{H}(i)}{\beta_{H}\left(I_{H}\right)}\right) \mathrm{d} i+\int_{i \in I_{O}} \ln \left(\frac{\zeta(i)}{\beta_{O}\left(I_{O}\right)}\right) \mathrm{d} i-\int_{0}^{1} \ln \left(a_{M}(i)\right) \mathrm{d} i\right)\right] .
$$


Next, recall the definition of the semi-elasticities of the comparative advantage schedules

$$
\varepsilon_{L}=-\frac{\partial \ln \beta_{L}\left(I_{L}\right)}{\partial I_{L}}>0, \quad \varepsilon_{H}=-\frac{\partial \ln \beta_{H}\left(I_{H}\right)}{\partial I_{H}}>0, \quad \frac{\partial \ln \beta_{O}\left(I_{O}\right)}{\partial I_{O}}=\mu>0 .
$$

Total differentiation w.r.t. the endogenous margins yields

$$
\begin{aligned}
\mathrm{d} \ln \Omega(\cdot)= & \ln \beta_{L}\left(I_{L}\right) \mathrm{d} I_{L}+I_{L} \frac{\beta_{L}^{\prime}\left(I_{L}\right)}{\beta_{L}\left(I_{L}\right)} \mathrm{d} I_{L}-\ln \beta_{L}\left(I_{L}\right) \mathrm{d} I_{L}-\ln \beta_{H}\left(I_{H}\right) \mathrm{d} I_{H}-\left(1-I_{H}\right) \frac{\beta_{H}^{\prime}\left(I_{H}\right)}{\beta_{H}\left(I_{H}\right)} \mathrm{d} I_{H} \\
& +\ln \beta_{H}\left(I_{H}\right) \mathrm{d} I_{H}+\left(\ln \zeta\left(I_{2}\right) \mathrm{d} I_{2}-\ln \zeta\left(I_{1}\right) \mathrm{d} I_{1}-\ln \beta_{O}\left(I_{O}\right) \mathrm{d} I_{O}\right)-I_{O} \frac{\beta_{O}^{\prime}\left(I_{O}\right)}{\beta_{O}\left(I_{O}\right)} \mathrm{d} I_{O} .
\end{aligned}
$$

Then, taking into account that the term within the brackets is, by the positive monotone transformation, discussed in Appendix 3.B.3, null, utilizing the definitions of semi-elasticity, and manipulating further, the total differentiation reduces to

$$
\mathrm{d} \ln \Omega(\cdot)=-I_{L} \varepsilon_{L} \mathrm{~d} I_{L}+\left(1-I_{H}\right) \varepsilon_{H} \mathrm{~d} I_{H}-I_{O} \mu \mathrm{d} I_{O} .
$$

Moreover, using the comparative static results derived in Appendix 3.D.1, we can compute the overall sign of $\mathrm{d} \ln \Omega(\cdot)$. Utilizing Eqs. (3.D.83) and (3.D.85), the internal reallocation effect, $\mathcal{R}=\left(1-I_{H}\right) \varepsilon_{H} \frac{\mathrm{d} I_{H}}{\mathrm{~d} \omega}-$ $I_{L} \varepsilon_{L} \frac{\mathrm{d} I_{L}}{\mathrm{~d} \omega}$, can be written as

$$
\begin{aligned}
\mathcal{R} & =\frac{\left[\left(1-I_{H}\right)^{2} \varepsilon_{H}\left(1+\varepsilon_{L} I_{L}\right)+I_{L}^{2} \varepsilon_{L}\left(1+\left(1-I_{H}\right) \varepsilon_{H}\right)\right]}{\alpha \omega I_{L}\left(1-I_{H}\right)\left(I_{H}-I_{L}-I_{O}\right) \Delta} \\
& =\frac{\left(1-I_{H}\right)^{2} \varepsilon_{H}+I_{L}^{2} \varepsilon_{L}+\left(1-I_{H}\right) I_{L} \varepsilon_{H} \varepsilon_{L}\left(1-\left(I_{H}-I_{L}\right)\right)}{\alpha \omega I_{L}\left(1-I_{H}\right)\left(I_{H}-I_{L}-I_{O}\right) \Delta} .
\end{aligned}
$$

Next, utilizing Eq. (3.D.83), the external reallocation effect, $\mathcal{E}=I_{O} \mu \frac{\mathrm{d} I_{O}}{\mathrm{~d} \omega}$, can be written as

$$
\mathcal{E}=\frac{I_{O} \mu\left[1-I_{O}+\varepsilon_{H}\left(1-I_{H}\right)\left[I_{H}-I_{O}+\varepsilon_{L} I_{L}\left(I_{H}-I_{L}-I_{O}\right)\right]+\varepsilon_{L} I_{L}\left(1-I_{L}-I_{O}\right)\right]}{\alpha \omega I_{L}\left(1-I_{H}\right)\left(I_{H}-I_{L}-I_{O}\right) \Delta} .
$$

Now from Eqs. (3.A.49) and (3.A.50), we can derive the sufficient condition that determines the sign of $\frac{\mathrm{d} \ln \Omega(\cdot)}{\mathrm{d} \omega}=\mathcal{R}-\mathcal{E} \lessgtr 0$.

$$
\begin{array}{r}
\left(1-I_{H}\right)^{2} \varepsilon_{H}+I_{L}^{2} \varepsilon_{L}+\left(1-I_{H}\right) I_{L} \varepsilon_{H} \varepsilon_{L}\left(1-\left(I_{H}-I_{L}\right)\right) \\
I_{O} \mu\left[1-I_{O}+\varepsilon_{H}\left(1-I_{H}\right)\left(I_{H}-I_{O}\right)+\varepsilon_{H}\left(1-I_{H}\right) \varepsilon_{L} I_{L}\left(I_{H}-I_{L}-I_{O}\right)+\varepsilon_{L} I_{L}\left(1-I_{L}-I_{O}\right)\right]
\end{array}
$$

It can be verified that for a sufficiently low substitutability (i.e. high values of $\mu$ ) between medium-skilled and offshore workers the marginal cost of composite labor unambiguously declines, i.e. $\frac{\mathrm{d} \ln \Omega(\cdot)}{\mathrm{d} \omega}<0$. Formally, the sufficient condition follows from the magnitude between the third term on the right hand side and the third term on the left hand side, which yields

$$
\mu>\frac{1-\left(I_{H}-I_{L}\right)}{I_{O}\left(I_{H}-I_{L}-I_{O}\right)} .
$$

Notice that if the sufficient condition (3.A.51) holds, the first two terms on the left hand side will also be dominated by the other terms on the right hand side. 


\section{B Proofs}

\section{B.1 Proof of Lemma 3.1}

Proof. We proceed as follows. A task is allocated to a low-skilled worker rather than to a medium-skilled worker as long as

$$
\begin{aligned}
c_{L}(i) & \leq c_{M}(i) \\
\Leftrightarrow \frac{w_{L}}{A_{L} a_{L}(i)} & \leq \frac{w_{M}}{A_{M} a_{M}(i)} \\
\Leftrightarrow \frac{w_{L}}{A_{L}} \frac{a_{M}(i)}{a_{L}(i)} & \leq \frac{w_{M}}{A_{M}},
\end{aligned}
$$

which, by Assumption 1, leads to Eq. (3.2) in Lemma 3.1 with $\beta_{L}\left(I_{L}\right)=\frac{a_{M}\left(I_{L}\right)}{a_{L}\left(I_{L}\right)}$. Similarly, tasks are allocated to medium-skilled workers as long as they are more productive relative to high-skilled workers. That is,

$$
\begin{aligned}
c_{M}(i) & \leq c_{H}(i) \\
\Leftrightarrow \frac{w_{M}}{A_{M} a_{M}(i)} & \leq \frac{w_{H}}{A_{H} a_{H}(i)} \\
\Leftrightarrow \frac{w_{M}}{A_{M}} & \leq \frac{a_{M}(i)}{a_{H}(i)} \frac{w_{H}}{A_{H}},
\end{aligned}
$$

which, by Assumption 1, leads to Eq. (3.3) in Lemma 3.1 with $\beta_{H}\left(I_{H}\right)=\frac{a_{M}\left(I_{H}\right)}{a_{H}\left(I_{H}\right)}$. Finally, with a similar argument firms allocate tasks between low and high skills according to

$$
\begin{aligned}
c_{L}(i) & \leq c_{H}(i) \\
\Leftrightarrow \frac{w_{L}}{A_{L} a_{L}(i)} & \leq \frac{w_{H}}{A_{H} a_{H}(i)} \\
\Leftrightarrow \frac{w_{L}}{A_{L}} \frac{a_{M}(i)}{a_{L}(i)} & \leq \frac{a_{M}(i)}{a_{H}(i)} \frac{w_{H}}{A_{H}},
\end{aligned}
$$

where, in the third inequality, we multiplied both sides by $a_{M}(i)$. By Assumption 1, we obtain Eq. (3.4) in Lemma 3.1 with $\beta_{L}(\tilde{I})=\frac{a_{M}(\tilde{I})}{a_{L}(\tilde{I})}$ and $\beta_{H}(\tilde{I})=\frac{a_{M}(\tilde{I})}{a_{H}(\tilde{I})}$.

\section{B.2 Proof of Lemma 3.2}

Similar to the discussion in Lemma 3.1, the allocation decision in Lemma 3.2 is based on cost efficiency. However, notice that by Assumption 2 there is a non-linear relationship between the unit costs of mediumskilled and offshore workers. Formally, the allocation problem can be written as

$$
\begin{aligned}
c_{M}(i) & \lesseqgtr c_{O}(i) \\
\Leftrightarrow \frac{w_{M}}{A_{M} a_{M}(i)} & \lesseqgtr \frac{\tau w_{O}}{A_{O} a_{O}(i)} \\
\Leftrightarrow \frac{w_{M}}{A_{M}} & \lesseqgtr \frac{\zeta(i)}{\omega},
\end{aligned}
$$

where $\frac{\partial \zeta(i)}{\partial i}<0, \forall i<\left.\check{I}\right|_{\frac{\partial \zeta(i)}{\partial i}=0}$ and $\frac{\partial \zeta(i)}{\partial i}>0, \forall i>\left.\check{I}\right|_{\frac{\partial \zeta(i)}{\partial i}=0}$. Thus, by the jointly necessary conditions in Corollary 2 there must exist two cut-off points at which $c_{M}(\cdot)-c_{O}(\cdot)=0$. These are defined by $(3.5)$ 
and (3.6), where

$$
\begin{aligned}
& \frac{w_{M}}{A_{M}}<\frac{\zeta(i)}{\omega}, \quad \forall i<I_{1} \text { and } i>I_{2} \\
& \frac{w_{M}}{A_{M}}>\frac{\zeta(i)}{\omega}, \quad \forall i \in\left(I_{1}, I_{2}\right) .
\end{aligned}
$$

The boundaries on $\omega$ in Corollary 2 defined as follows. The lower boundary follows from

$$
\begin{aligned}
c_{O}(\check{I}) & <c_{M}(\check{I}) \\
\Leftrightarrow \frac{A_{M}}{w_{M}} \zeta(\check{I}) & <\omega,
\end{aligned}
$$

with $\check{I}$ denoting the minimum point of $\zeta(i)$, i.e. $\frac{\partial \zeta(i)}{\partial i}=0$. In a similar vein, one can derive the upper boundaries, respectively, at the low- and high-skill-extensive margins, i.e. $c_{O}\left(I_{L}\right)>c_{M}\left(I_{L}\right)$ and $c_{O}\left(I_{H}\right)>c_{M}\left(I_{H}\right)$. Note again that the lower boundaries ensure that offshored job-tasks are a subset of the overall medium skill-intensive range of tasks, i.e. $\left(I_{1}, I_{2}\right) \in\left(I_{L}, I_{H}\right)$.

\section{B.3 Proof of Lemma 3.3}

To keep the analytical analysis tractable, it is useful to look at changes in the offshoring interval, which reflect implicitly changes in the extensive offshoring margins, $I_{1}$ and $I_{2}$. In fact, all we need to show is how an endogenous change in the length of offshoring interval affects the domestic job-task margins, $I_{L}$ and $I_{H}$. Thus we need to find a condition that satisfies the no-arbitrage condition between mediumskilled and offshore workers for the length of the offshoring interval $\left(I_{O}\right)$, accounting implicitly for the two endogenous offshoring cut-off points $I_{1}$ and $I_{2}$.

First, define $\tilde{w}_{M} \equiv \frac{w_{M} \omega}{A_{M}}$ after recalling the two no-arbitrage conditions (3.5) and (3.6)

$$
\begin{aligned}
\tilde{w}_{M} & =\zeta\left(I_{1}\right), \\
\tilde{w}_{M} & =\zeta\left(I_{2}\right),
\end{aligned}
$$

and the definition of the length of offshoring interval

$$
I_{O}=I_{2}-I_{1} .
$$

Next, recall the semi-elasticities at the two extensive offshoring margins, i.e. $\varepsilon_{1}=-\frac{\partial \ln \zeta\left(I_{1}\right)}{\partial I_{1}}>0$ and $\varepsilon_{2}=\frac{\partial \ln \zeta\left(I_{2}\right)}{\partial I_{2}}>0$. Taking the total differentiation, we obtain

$$
\begin{aligned}
\mathrm{d} \ln \tilde{w}_{M} & =-\varepsilon_{1} \mathrm{~d} I_{1}, \\
\mathrm{~d} \ln \tilde{w}_{M} & =\varepsilon_{2} \mathrm{~d} I_{2}, \\
\mathrm{~d} I_{O} & =\mathrm{d} I_{2}-\mathrm{d} I_{1} .
\end{aligned}
$$

Utilizing the first two equations in the last one yields

$$
\mathrm{d} I_{O}=\mathrm{d} \ln \tilde{w}_{M}\left(\frac{1}{\varepsilon_{2}}+\frac{1}{\varepsilon_{1}}\right) .
$$

It is convenient to define $\mu=\frac{\varepsilon_{2} \varepsilon_{1}}{\varepsilon_{2}+\varepsilon_{1}}>0$, which is increasing in both arguments. Then, after further manipulation, we obtain

$$
\mathrm{d} \ln \tilde{w}_{M}=\mu \mathrm{d} I_{O} .
$$

This is a simple first-order linear homogeneous ordinary differential equation. Thus, by integration

$$
\int \mathrm{d} \ln \tilde{w}_{M} \mathrm{~d} i=\int \mu \mathrm{d} I_{O} \mathrm{~d} i
$$


we obtain a unique solution

$$
\frac{w_{M}}{A_{M}}=\frac{\beta_{O}\left(I_{O}\right)}{\omega},
$$

where $\beta_{O}\left(I_{O}\right)=\exp \left[\mu I_{O}\right]$

\section{B.4 Proof of Proposition 3.1: Uniqueness of task margins}

To verify the uniqueness of the equilibrium task margins, we discuss the equilibrium properties of the domestic task margins from system (3.21). The proof of the claim regarding uniqueness of offshoring margins needs further elaboration. We commence with the equilibrium characteristics of the margins $I_{L}$ and $I_{H}$.

\section{Uniqueness of domestic task margins}

From (3.21a), we can rearrange so that

$$
I_{H}=\left(I_{2}-I_{1}\right)+\left(1+\mathcal{A}_{\mathcal{L}} \beta_{L}\left(I_{L}\right)\right) I_{L} \equiv I_{O}+\mathcal{F}_{L}\left(I_{L}\right) .
$$

Analogously, (3.21c) can be rearranged so that

$$
I_{L}=-\left(I_{2}-I_{1}\right)+I_{H}-\mathcal{A}_{\mathcal{H}} \beta_{H}\left(I_{H}\right)\left(1-I_{H}\right) \equiv-I_{O}+\mathcal{F}_{H}\left(I_{H}\right),
$$

where $\mathcal{A}_{\mathcal{L}} \equiv \frac{A_{M} N_{M}}{A_{L} N_{L}}$ and $\mathcal{A}_{\mathcal{H}} \equiv \frac{A_{M} N_{M}}{A_{H} N_{H}}$ summarize the exogenous labor-augmenting technology and labor endowment variables. Therefore Eqs. $\left(3.21 \mathrm{a}^{\prime}\right)$ and $\left(3.21 \mathrm{c}^{\prime}\right)$ highlight the general equilibrium relation between $I_{L}$ and $I_{H}$ for any given value of $I_{O} \in\left(I_{L}, I_{H}\right)$. Hence any changes in the offshoring extensive margins will shift $\left(3.21 \mathrm{a}^{\prime}\right)$ and $\left(3.21 \mathrm{c}^{\prime}\right)$, while changes in $I_{L}$ and $I_{H}$, captured by $\mathcal{F}_{k}(\cdot), k=\{L, H\}$, affect the slope. To verify the single-crossing between Eqs. $\left(3.21 \mathrm{a}^{\prime}\right)$ and $\left(3.21 \mathrm{c}^{\prime}\right)$, we need to examine the properties of the slope of the two curves.

Consider first the properties of Eq. $\left(3.21 \mathrm{a}^{\prime}\right)$. It can be shown that $I_{H}$ is monotonically increasing in $I_{L}$. Recalling the property $\beta_{L}^{\prime}\left(I_{L}\right)>0$, then the first derivative implies

$$
\frac{\partial \mathcal{F}_{L}}{\partial I_{L}}=1+\mathcal{A}_{\mathcal{L}}\left[\beta_{L}\left(I_{L}\right)+\beta_{L}^{\prime}\left(I_{L}\right) I_{L}\right]>0,
$$

indicating that the slope of $\left(3.21 \mathrm{a}^{\prime}\right)$ is larger than unity.

Moreover, it indicates that the increase at the low-skill-intensive task margin is accompanied by a reduction of high-skill-intensive tasks, i.e. lower $\left(1-I_{H}\right)$. Intuitively, this implies that the tasks previously performed by medium-skilled workers at $I_{L}$ are now produced by low-skilled workers. This induces an excess of the medium-skilled labor supply due to the Walrasian nature of the labor markets. Thus medium-skilled wages will decline, so that they become more competitive at the high-skill-extensive task margin $I_{H}$. This is then accompanied by increase in $I_{H}$.

The monotonic relation between $I_{L}$ and $I_{H}$ depends on the properties of the second derivative, which in turn depends on the functional properties of the productivity schedule $\beta_{L}(\cdot)$. To examine these properties, we proceed as follows. Let $\beta_{L}(i)$ be a homogeneous function of degree $1 \geq \lambda_{L}>0$, indicating a concave function. Then, it is generally valid $\left|\beta_{L}^{\prime}(i)\right|>\left|\beta_{L}^{\prime \prime}(i)\right|$. If, on the other hand, $\beta_{L}(i)$ is homogeneous of degree $\lambda_{L}>1$ (indicating a convex function), then $0<\beta_{L}^{\prime}(i)<\beta_{L}^{\prime \prime}(i)$. Thus, for any functional property of $\beta_{L}(\cdot)$, the second derivative yields $\frac{\partial^{2} \mathcal{F}_{L}}{\partial I_{L}^{2}}=\mathcal{A}_{\mathcal{L}}\left(2 \beta_{L}^{\prime}\left(I_{L}\right)+\beta_{L}^{\prime \prime}\left(I_{L}\right) I_{L}\right)>0$. This indicates that $\mathcal{F}_{L}$ is monotonically increasing in $I_{L}$. Furthermore, computing the limits of $\mathcal{F}_{L}$ over the range defined by 
Corollaries 1 and 2, yields

$$
\begin{gathered}
\lim _{I_{L} \rightarrow 0} \mathcal{F}_{L}=0 \Rightarrow \lim _{I_{L} \rightarrow 0} I_{H}=I_{2}-I_{1}=I_{O} \\
\lim _{I_{L} \rightarrow I_{1}} \mathcal{F}_{L}=I_{1}+\mathcal{A}_{\mathcal{L}} \beta_{L}\left(I_{1}\right) I_{1} \Rightarrow \lim _{I_{L} \rightarrow I_{1}} I_{H}=I_{2}+\mathcal{A}_{\mathcal{L}} \beta_{L}\left(I_{1}\right) I_{1} .
\end{gathered}
$$

Next consider Eq. $\left(3.21 \mathrm{c}^{\prime}\right)$. Similarly, it can be verified that $I_{L}$ is monotonically increasing in $I_{H}$, again for any (fixed) values of $I_{O} \in\left(I_{L}, I_{H}\right)$. Recall the property $\beta_{H}^{\prime}\left(I_{H}\right)<0$, then formally

$$
\frac{\partial \mathcal{F}_{H}}{\partial I_{H}}=1+\mathcal{A}_{\mathcal{H}}\left[\beta_{H}\left(I_{H}\right)-\beta_{H}^{\prime}\left(I_{H}\right)\left(1-I_{H}\right)\right]>0,
$$

implying a slope of $\left(3.21 c^{\prime}\right)$ larger than unity. In a similar vein, if $\beta_{H}(i)$ is homogeneous of degree $-1<$ $\lambda_{H}<0$ (i.e. a concave functional form), then $\beta_{H}^{\prime \prime}(i)<\beta_{H}^{\prime}(i)<0$. If $\beta_{H}(i)$ is a homogeneous function of degree $\lambda_{H}<-1$ (indicating a convex functional form), then $\beta_{H}^{\prime}(i)<0<\beta_{H}^{\prime \prime}(i)$. Thus, computing the second derivative yields $\frac{\partial^{2} \mathcal{F}_{H}}{\partial I_{H}^{2}}=\mathcal{A}_{\mathcal{H}}\left(2 \beta_{H}^{\prime}\left(I_{H}\right)-\beta_{H}^{\prime \prime}\left(I_{H}\right)\left(1-I_{H}\right)\right)<0$. Similarly, computing the limits of $\mathcal{F}_{H}$ over the range defined by Corollaries 1 and 2 , we get

$$
\begin{aligned}
\lim _{I_{H} \rightarrow I_{2}} \mathcal{F}_{H}=I_{2}-\mathcal{A}_{\mathcal{H}} \beta_{H}\left(I_{2}\right)\left(1-I_{2}\right) & \Rightarrow \lim _{I_{H} \rightarrow I_{2}} I_{L}=-\left(\mathcal{A}_{\mathcal{H}} \beta_{H}\left(I_{2}\right)\left(1-I_{2}\right)-I_{1}\right) \\
\lim _{I_{H} \rightarrow 1} \mathcal{F}_{H}=1 & \Rightarrow \lim _{I_{H} \rightarrow 1} I_{L}=1-\left(I_{2}-I_{1}\right) .
\end{aligned}
$$

Utilizing the properties of $\mathcal{F}_{L}$ and $\mathcal{F}_{H}$ derived in (5.30) and (5.31), we can depict Eqs. $\left(3.21 \mathrm{a}^{\prime}\right)$ and $\left(3.21 \mathrm{c}^{\prime}\right)$ in the $\left(I_{L}, I_{H}\right)$-space. The graphical illustration in Figure 3.5 reveals the single crossing between the two curves for all values within the bounded intervals.

Obviously, if the offshoring range becomes very large such that $I_{O} \notin\left(I_{L}, I_{H}\right)$, then the intersection of the two curves, given by Eqs. $\left(3.21 \mathrm{a}^{\prime}\right)$ and $\left(3.21 \mathrm{c}^{\prime}\right)$, will be off the unit interval. More precisely, an increase

Figure 3.5: Unique equilibrium of low- and high-skilled task margins

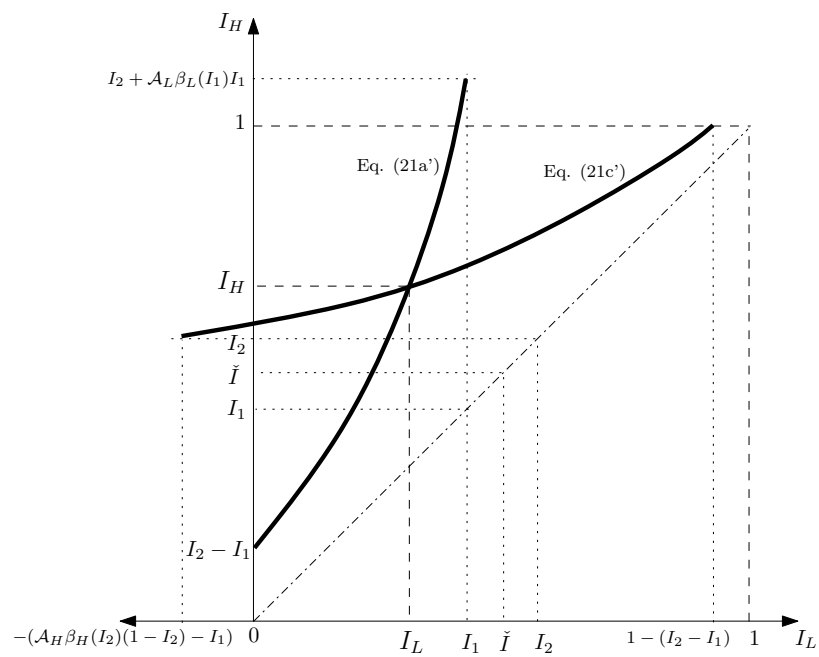

in $I_{O}=\left(I_{2}-I_{1}\right)$ induces a parallel left-ward shift in the two curves implying unambiguously an increase in $I_{H}$ and a decline in $I_{L} \cdot{ }^{21}$ The fact that there is a parallel shift can be seen by computing the limits of

\footnotetext{
${ }^{21}$ Since the two curves have different slopes in absolute values, any parallel shift induces an
} 
$\left(3.21 \mathrm{a}^{\prime}\right)$ and $\left(3.21 \mathrm{c}^{\prime}\right)$ over the unit interval. Formally this is given by $\lim _{I_{L} \rightarrow 1} I_{H}=1+\left(I_{2}-I_{1}\right)+\mathcal{A}_{\mathcal{L}} \beta_{L}(1)$ and $\lim _{I_{H} \rightarrow 0} I_{H}=-\left(\left(I_{2}-I_{1}\right)+\mathcal{A}_{\mathcal{H}} \beta_{H}(0)\right.$. Thus it is readily evident that changes in the set of offshorable tasks lead to a shift in both curves, whereas changes in factor-biased technology or endowments captured by the terms $\mathcal{A}_{\mathcal{L}}$ and $\mathcal{A}_{\mathcal{H}}$ will also affect the slope of both curves. We summarize by the following Lemma the first sufficient condition.

Lemma 3.4. For any given values of $I_{O} \in\left(I_{L}, I_{H}\right)$, Eqs. $\left(3.21 \mathrm{a}^{\prime}\right)$ and $\left(3.21 \mathrm{c}^{\prime}\right)$ determine the unique values of $I_{L}$ and $I_{H}$ in the $\left(I_{L}, I_{H}\right)$-space.

\section{Unique solution of offshoring task margins}

In order to assess the uniqueness of the offshoring task margins, we need to account explicitly for them. In doing so, recall the offshoring no-arbitrage conditions (3.5) and (3.6). Combining these conditions with the equilibrium relative demand conditions, we obtain

$$
\begin{aligned}
\frac{\mathcal{B}}{N_{M} A_{M}} \omega^{\frac{1}{\alpha}} & =\frac{\zeta_{1}\left(I_{1}\right)^{\frac{1}{\alpha}} \Omega(\cdot)^{\frac{1-\alpha}{\alpha}}}{I_{H}-I_{L}-\left(I_{2}-I_{1}\right)} \\
\frac{\mathcal{B}}{N_{M} A_{M}} \omega^{\frac{1}{\alpha}} & =\frac{\zeta_{2}\left(I_{2}\right)^{\frac{1}{\alpha}} \Omega(\cdot)^{\frac{1-\alpha}{\alpha}}}{I_{H}-I_{L}-\left(I_{2}-I_{1}\right)} .
\end{aligned}
$$

The equilibrium properties of the offshoring margins can be assessed as follows. For all $I_{L} \in\left(0, I_{2}\right)$ and $I_{H} \in\left(I_{2}, 1\right)$, Eqs. (3.B.54) and (3.B.55) determine the equilibrium values of $I_{1}$ and $I_{2}$. However, notice that, due to the non-linearity, we derive the implicit solution by means of the Implicit Function Theorem (IFT).

Consider first Eq. (3.B.54). Rearranging slightly yields

$$
\mathcal{A}_{\mathcal{O}}\left(I_{H}-I_{L}-\left(I_{2}-I_{1}\right)\right)=\zeta\left(I_{1}\right)^{\frac{1}{\alpha}}(\Omega(\cdot))^{\frac{1-\alpha}{\alpha}},
$$

with $\mathcal{A}_{\mathcal{O}} \equiv \frac{\mathcal{B}}{N_{M} A_{M}} \omega^{\frac{1}{\alpha}}$.

Take logs in (3.B.54') and define

$$
\mathcal{G}\left(I_{1}, I_{2}\right) \equiv \ln \mathcal{A}_{\mathcal{O}}+\ln \left(I_{H}-I_{L}-\left(I_{2}-I_{1}\right)\right)-\frac{1}{\alpha} \ln \zeta\left(I_{1}\right)-\frac{1-\alpha}{\alpha} \ln \Omega\left(I_{1}, I_{2}, \cdot\right)=0 .
$$

Then, taking for the moment $I_{L}$ and $I_{H}$ as given, by IFT we obtain

$$
\frac{\mathrm{d} I_{2}}{\mathrm{~d} I_{1}}=-\frac{\mathcal{G}_{I_{1}}\left(I_{1}, I_{2}\right)}{\mathcal{G}_{I_{2}}\left(I_{1}, I_{2}\right)},
$$

where $\mathcal{G}_{I_{1}}\left(I_{1}, I_{2}\right)$ and $\mathcal{G}_{I_{2}}\left(I_{1}, I_{2}\right)$ denote the partial derivatives w.r.t. the extensive margins and are defined by

$$
\begin{aligned}
\frac{\partial \mathcal{G}\left(I_{1}, I_{2}\right)}{\partial I_{1}} & =\frac{1}{I_{H}-I_{L}-\left(I_{2}-I_{1}\right)}-\frac{1}{\alpha} \frac{\zeta_{I_{1}}\left(I_{1}\right)}{\zeta\left(I_{1}\right)}-\frac{1-\alpha}{\alpha} \frac{\Omega_{I_{1}}(\cdot)}{\Omega(\cdot)} \\
\frac{\partial \mathcal{G}\left(I_{1}, I_{2}\right)}{\partial I_{2}} & =-\frac{1}{I_{H}-I_{L}-\left(I_{2}-I_{1}\right)}-\frac{1-\alpha}{\alpha} \frac{\Omega_{I_{2}}(\cdot)}{\Omega(\cdot)}
\end{aligned}
$$

unambiguous change in both margins. 
where $^{22}$

$$
\frac{\Omega_{I_{1}}(\cdot)}{\Omega(\cdot)}=-\left(\check{I}-I_{1}\right) \frac{\zeta_{I_{1}}\left(I_{1}\right)}{\zeta\left(I_{1}\right)}, \quad \frac{\Omega_{I_{2}}(\cdot)}{\Omega(\cdot)}=-\left(I_{2}-\check{I}\right) \frac{\zeta_{I_{2}}\left(I_{2}\right)}{\zeta\left(I_{2}\right)},
$$

with $\zeta_{j}(j)$ denoting the partial derivative w.r.t. to $j=\left\{I_{1}, I_{2}\right\}$. Note also that the IFT requires $\mathcal{G}_{I_{2}}\left(I_{1}, I_{2}\right) \neq 0$. Recalling the definition of the elasticities of the task productivities at the extensive offshore margins $\tilde{\varepsilon}_{\zeta_{1}}=-\frac{\zeta_{I_{1}}\left(I_{1}\right)}{\zeta\left(I_{1}\right)} I_{1}>0$ and $\varepsilon_{\zeta_{2}}=\frac{\zeta_{I_{2}}\left(I_{2}\right)}{\zeta\left(I_{2}\right)} I_{2}>0$, then utilizing the solutions of the partial derivatives in (3.B.56), we get

$$
\frac{\mathrm{d} I_{2}}{\mathrm{~d} I_{1}}=-\left(\frac{\frac{I_{1}}{I_{H}-I_{L}-\left(I_{2}-I_{1}\right)}+\left(1-(1-\alpha)\left(\check{I}-I_{1}\right)\right) \frac{\tilde{\varepsilon}_{\zeta_{1}}}{\alpha}}{-\frac{I_{2}}{I_{H}-I_{L}-\left(I_{2}-I_{1}\right)}+(1-\alpha)\left(I_{2}-\check{I}\right) \frac{\varepsilon_{\zeta_{2}}}{\alpha}} \frac{I_{2}}{I_{1}}\right) .
$$

Rearranging slightly yields

$$
\frac{\hat{I}_{2}}{\hat{I}_{1}}=\left(\frac{\alpha I_{1}+\left(1-(1-\alpha)\left(\check{I}-I_{1}\right)\right)\left(I_{H}-I_{L}-\left(I_{2}-I_{1}\right)\right) \tilde{\varepsilon}_{\zeta_{1}}}{\alpha I_{2}-(1-\alpha)\left(I_{2}-\check{I}\right)\left(I_{H}-I_{L}-\left(I_{2}-I_{1}\right)\right) \varepsilon_{\zeta_{2}}}\right) \equiv q\left(I_{1}, I_{2}, \cdot\right),
$$

where $\hat{x} \equiv \frac{\mathrm{d} x}{x}$ denotes the rate of change. Thus, the right hand side of $\left(3 . \mathrm{B} .56^{\prime}\right), q\left(I_{1}, I_{2}\right)$, denotes the elasticity.

We now turn to the implicit behavior of Eq. (3.B.55). Rearrange this equation to obtain

$$
\mathcal{A}_{\mathcal{O}}\left(I_{H}-I_{L}-\left(I_{2}-I_{1}\right)\right)=\zeta\left(I_{2}\right)^{\frac{1}{\alpha}}(\Omega(\cdot))^{\frac{1-\alpha}{\alpha}} .
$$

Now, following the same steps considered for the derivation of $\left(3 . B .56^{\prime}\right)$, we get a second implicit relation between $I_{2}$ and $I_{1}$. Formally, it is given by

$$
\frac{\mathrm{d} I_{2}}{\mathrm{~d} I_{1}}=-\left(\frac{-\frac{I_{1}}{\left(I_{H}-I_{L}-\left(I_{2}-I_{1}\right)\right)}+(1-\alpha)\left(\check{I}-I_{1}\right) \frac{\tilde{\varepsilon}_{\zeta_{1}}}{\alpha}}{\frac{I_{2}}{\left(I_{H}-I_{L}-\left(I_{2}-I_{1}\right)\right)}+\left(1-(1-\alpha)\left(I_{2}-\check{I}\right)\right) \frac{\varepsilon_{\zeta_{2}}}{\alpha}} \frac{I_{2}}{I_{1}}\right) .
$$

Rearranging slightly, yields

$$
\frac{\hat{I}_{2}}{\hat{I}_{1}}=\left(\frac{\alpha I_{1}-(1-\alpha)\left(\check{I}-I_{1}\right)\left(I_{H}-I_{L}-\left(I_{2}-I_{1}\right)\right) \tilde{\varepsilon}_{\zeta_{1}}}{\alpha I_{2}+\left(1-(1-\alpha)\left(I_{2}-\check{I}\right)\right)\left(I_{H}-I_{L}-\left(I_{2}-I_{1}\right)\right) \varepsilon_{\zeta_{2}}}\right) \equiv z\left(I_{1}, I_{2}, \cdot\right),
$$

where the right-hand side, $z\left(I_{1}, I_{2}\right)$, denotes the elasticity between $I_{2}$ and $I_{1}$.

In order to assess the behavior of the two implicit relations derived in $\left(3 . B .56^{\prime}\right)$ and (3.B.57'), we need to elaborate on the sign of the elasticities. It is evident that the sign of the two elasticities $q(\cdot)$ and $z(\cdot)$ depends, respectively, on the sign of the denominator and the numerator. That is, from (3.B.56')

$$
q(\cdot)=\left\{\begin{array}{cl}
>0 & \text { if } \frac{\alpha}{1-\alpha} \frac{I_{2}}{\left(I_{2}-\check{I}\right)\left(I_{H}-I_{L}-\left(I_{2}-I_{1}\right)\right)}>\varepsilon_{\zeta_{2}} \\
<0 & \text { if } \frac{\alpha}{1-\alpha} \frac{I_{2}}{\left(I_{2}-\check{I}\right)\left(I_{H}-I_{L}-\left(I_{2}-I_{1}\right)\right)}<\varepsilon_{\zeta_{2}} .
\end{array}\right.
$$

Similarly, from (3.B. $\left.57^{\prime}\right)$

$$
z(\cdot)= \begin{cases}>0 & \text { if } \frac{\alpha}{1-\alpha} \frac{I_{1}}{\left(\check{I}-I_{1}\right)\left(I_{H}-I_{L}-\left(I_{2}-I_{1}\right)\right)}>\tilde{\varepsilon}_{\zeta_{1}} \\ <0 & \text { if } \frac{\alpha}{1-\alpha} \frac{I_{1}}{\left(\breve{I}-I_{1}\right)\left(I_{H}-I_{L}-\left(I_{2}-I_{1}\right)\right)}<\tilde{\varepsilon}_{\zeta_{1}} .\end{cases}
$$

Thus the magnitude (in absolute values) of the elasticities of the task productivity schedules at the

\footnotetext{
${ }^{22}$ The derivative of $\Omega(\cdot)$ w.r.t. any task margin can be computed considering the following general case: if $f(x, \cdot)=\exp [g(x)+\ldots]$, then $\partial f(x) / \partial x=g^{\prime}(x) f(x)$. Here, $g\left(I_{1}\right)=\int_{I_{1}}^{\check{I}} \ln \left(\frac{\zeta(i)}{\zeta\left(I_{1}\right)}\right) \mathrm{d} i$ and $g^{\prime}\left(I_{1}\right)=-\ln \zeta\left(I_{1}\right)-\left(\check{I}-I_{1}\right) \frac{\zeta_{I_{1}}\left(I_{1}\right)}{\zeta\left(I_{1}\right)}+\ln \zeta\left(I_{1}\right)=-\left(\check{I}-I_{1}\right) \frac{\zeta_{I_{1}}\left(I_{1}\right)}{\zeta\left(I_{1}\right)}$.
} 
respective extensive margins determines the implicit relation between $I_{2}$ and $I_{1}$. Put differently, it is obvious that, if $q(\cdot)$ and $z(\cdot)$ have opposite signs, there must be a single crossing in the $\left(I_{1}, I_{2}\right)$ space, for all $I_{L} \in\left(0, I_{1}\right), I_{H} \in\left(I_{2}, 1\right)$. Thus, when both have equal signs it is important to verify that one of the elasticities is larger (in absolute values). In doing so, define $\alpha_{1} \equiv \alpha I_{1}, \alpha_{2} \equiv \alpha I_{2}$, $\check{\alpha} \equiv(1-\alpha)\left(I_{H}-I_{L}-\left(I_{2}-I_{1}\right)\right), \mathcal{S}_{\mathcal{M}} \equiv\left(I_{H}-I_{L}-\left(I_{2}-I_{1}\right)\right)$, then it can be shown that

$$
\begin{aligned}
|q(\cdot)| & >|z(\cdot)| \\
\left|\left(\frac{\left.\alpha_{1}+\left(\mathcal{S}_{\mathcal{M}}-\left(\check{I}-I_{1}\right) \check{\alpha}\right)\right) \tilde{\varepsilon}_{\zeta_{1}}}{\alpha_{2}-\left(I_{2}-\check{I}\right) \check{\alpha} \varepsilon_{\zeta_{2}}}\right)\right| & >\left|\left(\frac{\alpha_{1}-\left(\check{I}-I_{1}\right) \check{\alpha} \tilde{\varepsilon}_{\zeta_{1}}}{\alpha_{2}+\left(\mathcal{S}_{\mathcal{M}}-\check{\alpha}\left(I_{2}-\check{I}\right)\right) \varepsilon_{\zeta_{2}}}\right)\right| \\
{\left.\left[\alpha_{2}+\left(\mathcal{S}_{\mathcal{M}}-\check{\alpha}\left(I_{2}-\check{I}\right)\right) \varepsilon_{\zeta_{2}}\right]\left[\alpha_{1}+\left(\mathcal{S}_{\mathcal{M}}-\left(\check{I}-I_{1}\right) \check{\alpha}\right)\right) \tilde{\varepsilon}_{\zeta_{1}}\right] } & >\left(\alpha_{1}-\left(\check{I}-I_{1}\right) \check{\alpha} \tilde{\varepsilon}_{\zeta_{1}}\right)\left(\alpha_{2}-\left(I_{2}-\check{I}\right) \check{\alpha} \varepsilon_{\zeta_{2}}\right) \\
\mathcal{S}_{\mathcal{M}}\left(\alpha_{1} \varepsilon_{\zeta_{2}}+\alpha_{2} \tilde{\varepsilon}_{\zeta_{1}}\right) & >0 .
\end{aligned}
$$

The next lemma summarizes the second sufficient condition. ${ }^{23}$

Lemma 3.5. For any values of $I_{L} \in\left(0, I_{1}\right)$ and $I_{H} \in\left(I_{2}, 1\right)$, the sufficient conditions by the Existence and Uniqueness Theorem (EUT) state that for all values in the intervals $I_{1} \in\left(I_{L}, \check{I}\right)$ and $I_{2} \in\left(\check{I}, I_{H}\right)$ (i) a solution exists if $q(\cdot)$ and $z(\cdot)$ are continuous, and (ii) the solution is unique if both $\frac{\partial q(\cdot)}{\partial I_{2}}$ and $\frac{\partial z(\cdot)}{\partial I_{2}}$ are also continuous. Then, there exist unique values of $I_{1}$ and $I_{2}$ in the $\left(I_{1}, I_{2}\right)$-space.

Thus Lemmas 3.4 and 3.5 establish the sufficient conditions for the uniqueness of the equilibrium values of the four endogenous margins.

\section{C Extension: Alternative offshoring scenarios of domestic job tasks}

\section{C.1 Offshoring low-skill-intensive domestic job tasks}

This section provides an analytical discussion of different stages of globalization trends. Particularly, we discuss the distributional effect when offshoring is limited to other skill segments of domestic tasks, while keeping the structure of the model unchanged. We commence with the special case as in Grossman and Rossi-Hansberg (2008), where offshoring activities are limited to low-skill-intensive job tasks. We refer to this case as globalization trends in the past, where the burden was mainly borne by low-skilled workers in many advanced countries. We then assess the distributional effects of easier offshoring.

First, notice that domestic skill-task allocations defined by Lemma 3.1 still hold. Then, similar to the case of offshoring medium-skilled tasks, we impose that the task assignment between low-skilled and offshore workers will lead to offshoring of a fraction of all low-skilled job tasks in the interval $\left(0, I_{L}\right)$. This implies that domestic firms find it cheaper to install low-skilled workers at the lower and upper ends of all low-skill-intensive job tasks, while offshoring is cheaper somewhere in the middle. Formally, the assignment problem is defined by

$$
c_{O}(i) \lesseqgtr c_{L}(i)
$$

or equivalently

$$
\frac{\tau w_{O}}{A_{O}} \frac{1}{a_{O}(i)} \lesseqgtr \frac{w_{L}}{A_{L}} \frac{1}{a_{L}(i)}, \quad \forall i \in I_{L} \backslash \tilde{I}_{O} .
$$

Multiplying both sides of (3.C.60) by $a_{M}(i)$ yields the familiar structure of task productivity schedules, i.e. $\beta_{L}(i)=\frac{a_{L}(i)}{a_{M}(i)}$ and $\tilde{\zeta}(i)=\frac{a_{M}(i)}{a_{O}(i)}$, with the functional properties defined by Assumptions 1 and 2 in the main text. Notice the difference between the task productivity schedule $\tilde{\zeta}(i)$ (imposed here) and $\zeta(i)$ (used previously). While both have the U-shaped functional form, $\tilde{\zeta}(i)$ indicates a different location over

\footnotetext{
${ }^{23}$ For a general discussion of the Existence and Uniqueness Theorem see Gandolfo (2010, Ch. 23.1.1).
} 
the unit interval. To fix ideas, consider, for example, the following simple quadratic function

$$
\zeta(i)=\exp \left[g_{M}\left(i-g_{O}\right)^{2}\right]
$$

where $g_{M}$ and $g_{O}$ are parameters and measure the task productivity of medium-skilled and offshore workers respectively. In this example, it can be easily verified that changes in the task productivity of offshore workers $\left(g_{O}\right)$ will induce a horizontal shift of $\zeta(i)$, changes in $g_{M}$ affect the slope, while lower offshoring friction (i.e. higher values of $\left.\omega \equiv \frac{A_{O}}{\tau w_{O}}\right)$ leads to a vertical shift of $\zeta(i) / \omega$. Notice however that if the comparative advantage of offshore workers is sufficiently low (i.e. $g_{O}=0$ ), there are no domestic low-skilled workers employed near the origin, and if they have sufficiently high task productivity (i.e. $\left.g_{O}=I_{L}\right)$, there are no low-skilled workers employed between the offshoring interval and the task margin $I_{L}$. Thus, as derived below, necessary conditions similar to those derived in Corollary 2 are required for which $\tilde{\zeta}(i) \in\left(0, I_{L}\right)$.

The necessary conditions for offshoring activities to be permitted to a sub-range of low-skilled job tasks are

$$
\frac{A_{L}}{w_{L}} \beta_{L}(\dot{I}) \tilde{\zeta}(\dot{I})<\omega<\frac{A_{L}}{w_{L}} \beta_{L}\left(I_{L}\right) \tilde{\zeta}\left(I_{L}\right), \quad \text { and } \quad 0<\left.\tilde{I}\right|_{\frac{\partial \ln \tilde{\zeta}(\cdot)}{\partial i}=0}<I_{L},
$$

implying that the offshoring friction is not too large (i.e. low values of $\omega=\frac{A_{O}}{\tau w_{O}}$ ), defined at the tangent point $\dot{I}$ between the productivity schedules $\left|\frac{\partial \beta_{L}(\dot{I})}{\partial \dot{I}}\right|=\left|\frac{\partial \tilde{\zeta}(\dot{I})}{\partial \dot{I}}\right|$, such that offshoring is permitted, nor too small, avoiding that low-skilled workers lose their comparative advantage in competing with mediumskilled workers, defined by the equilibrium task margin $I_{L} \cdot{ }^{24}$

Then, given Assumptions 1 and 2, and conditions in (3.C.61), it follows from (3.C.60) that there must exist two cut-off margins, similar to Lemma 3.2, defining the range of offshored low-skilled job tasks, i.e. $\tilde{I}_{O}=\tilde{I}_{2}-\tilde{I}_{1}$.

However, as discussed in the main text, by means of simple positive monotone transformation (see Lemma 3.3 ), we obtain

$$
\frac{\tilde{\beta}_{O}\left(\tilde{I}_{O}\right)}{\omega}=\frac{w_{L}}{A_{L}}
$$

where $\tilde{\beta}_{O}\left(I_{O}\right)$ is the positive monotone transformation of comparative advantage schedules $\tilde{\zeta}(\cdot) \beta_{L}(\cdot)$ at the new task margins, $\tilde{I}_{1}$ and $\tilde{I}_{2}$, in terms of the offshoring range $\tilde{I}_{O}$.

\section{Figure 3.6: Offshorability of low-skill-intensive job-tasks and skill-task allocation}

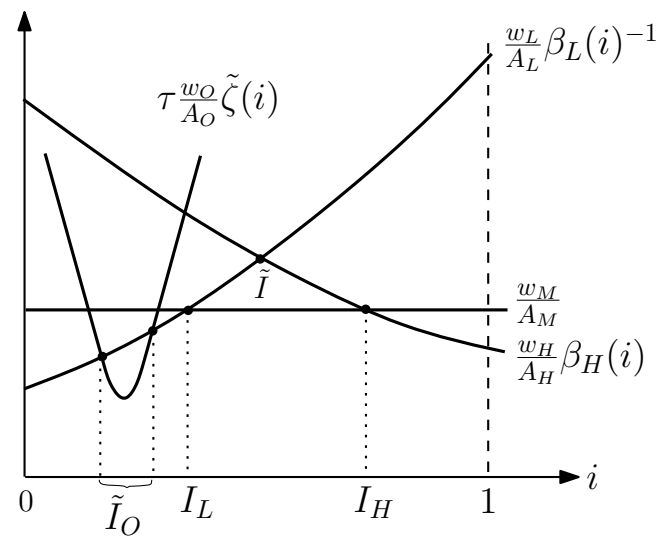

\footnotetext{
${ }^{24}$ It is important to notice that at the tangent point $\dot{I}$, the domestic firm is indifferent between lowskilled and offshore workers. Thus, for a positive range of offshoring low-skilled job tasks, $\dot{I}_{O}>0$, the offshoring friction must be sufficiently low.
} 
Thus the allocation of tasks is as follows: low-skilled workers perform all tasks $i \in I_{L} \backslash \tilde{I}_{O}$ and offshore workers perform all tasks $i \in \tilde{I}_{O}$. The task allocations between low- and medium-skilled workers and between high- and medium-skilled workers still hold as defined in Lemma 3.1, where medium-skilled workers perform all tasks $i \in\left(I_{L}, I_{H}\right)$, while high-skilled workers perform all tasks $i \in\left[I_{H}, 1\right]$. Figure 3.6 provides a graphical illustration.

\section{C.2 General equilibrium solution}

Equipped with these conditions, we derive the general equilibrium closed solution as follows. From the cost-minimized first-order conditions, we obtain three equations denoting the relative medium-skilled labor demand

$$
\begin{aligned}
\frac{w_{L}}{w_{M}} & =\frac{N_{M}}{N_{L}} \frac{I_{L}-\tilde{I}_{O}}{I_{H}-I_{L}} \\
\frac{w_{O}}{w_{M}} & =\frac{N_{M}}{n_{O}} \frac{\tilde{I}_{O}}{I_{H}-I_{L}} \\
\frac{w_{M}}{w_{H}} & =\frac{N_{M}}{N_{H}} \frac{I_{H}-I_{L}}{1-I_{H}} .
\end{aligned}
$$

Recall the no-arbitrage conditions (3.2) and (3.3)

$$
\begin{aligned}
\frac{w_{L}}{w_{M}} & =\frac{A_{L}}{A_{M}} \beta_{L}\left(I_{L}\right) \\
\frac{w_{M}}{w_{H}} & =\frac{A_{M}}{A_{H}} \beta_{H}\left(I_{H}\right) .
\end{aligned}
$$

To examine changes in the relative demand between medium-skilled and offshore workers, the noarbitrage condition (3.C.62) has to be adjusted for medium-skill unit costs. Thus, dividing both sides of Eq. (3.C.62) by $w_{M}$ and using the no-arbitrage condition at $I_{L}$, Eq. (3.2), we obtain

$$
\frac{w_{O}}{w_{M}}=\frac{A_{O}}{\tau} \frac{\beta_{L}\left(I_{L}\right)}{A_{M}} \frac{1}{\tilde{\beta}_{O}\left(\tilde{I}_{O}\right)} .
$$

Moreover, notice the additional endogenous variable in Eq. (3.C.64): $n_{O}$, i.e. the employment level of offshore workers. To account for the endogenous adjustment of offshore employment level, combine the first-order condition for offshore labor per task with the resource constraint for offshore workers to obtain

$$
\frac{n_{O}}{\tilde{I}_{O}}=\frac{P_{E} E}{w_{O}}=\frac{P_{E}^{-\frac{1-\alpha}{\alpha}} \mathcal{B}}{w_{O}}=\frac{\mathcal{B}}{w_{O}}\left(\beta_{L}\left(I_{L}\right)^{-1} \frac{\tau w_{O}}{A_{O}} \tilde{\beta}_{O}\left(\tilde{I}_{O}\right) \tilde{\Omega}(\cdot)\right)^{-\frac{1-\alpha}{\alpha}}
$$

where the second equality accounts for the adjustment in total employment $E=P_{E}^{-1 / \alpha} \mathcal{B}$. In the third equality we utilized the modified no-arbitrage condition (3.C.67) together with the general equilibrium solution of the price index $P_{E}=\frac{w_{M}}{A_{M}} \tilde{\Omega}(\cdot)$, where

$$
\tilde{\Omega}(\cdot)=\exp \left[\int_{i \in \tilde{I}_{O}} \ln \left(\frac{\beta_{L}(i) \tilde{\zeta}(i)}{\tilde{\beta}_{O}\left(\tilde{I}_{O}\right)}\right) \mathrm{d} i+\int_{0}^{I_{L}} \ln \left(\frac{\beta_{L}\left(I_{L}\right)}{\beta_{L}(i)}\right) \mathrm{d} i+\int_{I_{H}}^{1} \ln \left(\frac{\beta_{H}(i)}{\beta_{H}\left(I_{H}\right)}\right) \mathrm{d} i-\int_{0}^{1} \ln a_{M}(i) \mathrm{d} i\right] .
$$

Moreover, the convenient structure of the generalized common part of the marginal cost of factor labor $\tilde{\Omega}(\cdot)$ is preserved. To see this, define the semi-elasticities at the offshoring margin $\tilde{\mu} \equiv \frac{\partial \ln \tilde{\beta}_{O}\left(\tilde{I}_{O}\right)}{\partial \tilde{I}_{O}}>0$. 
Taking the total differentiation w.r.t. the endogenous task margins yields

$$
\begin{aligned}
\mathrm{d} \ln \tilde{\Omega}(\cdot)= & \left(\ln \tilde{\zeta}\left(\tilde{I}_{O}\right)+\beta_{L}\left(\tilde{I}_{O}\right)-\ln \tilde{\beta}_{O}\left(\tilde{I}_{O}\right)\right) \mathrm{d} \tilde{I}_{O}-\tilde{I}_{O} \tilde{\mu} \mathrm{d} \tilde{I}_{O} \\
& +\left(\ln \beta_{L}\left(I_{L}\right)-\ln \beta_{L}\left(I_{L}\right)\right) \mathrm{d} I_{L}-I_{L} \varepsilon_{L} \mathrm{~d} I_{L} \\
& +\left(\ln \beta_{H}\left(I_{H}\right)-\ln \beta_{H}\left(I_{H}\right)\right) \mathrm{d} I_{H}+\left(1-I_{H}\right) \varepsilon_{H} \mathrm{~d} I_{H}
\end{aligned}
$$

By the positive monotone transformation the bracket in the first line is zero. The brackets in the second and third lines are also zero, yielding

$$
\mathrm{d} \ln \tilde{\Omega}(\cdot)=\left(1-I_{H}\right) \varepsilon_{H} \mathrm{~d} I_{H}-I_{L} \varepsilon_{L} \mathrm{~d} I_{L}-\tilde{I}_{O} \tilde{\mu} \mathrm{d} \tilde{I}_{O}
$$

Thus, similar to the discussion in the main text, the impact on the generalized marginal cost of labor can be decomposed into three terms. As shown in Eq. (3.C.69), the direct source of the productivity effect due to offshoring is captured by $\tilde{I}_{O} \tilde{\mu} \mathrm{d} \tilde{I}_{O}$. The first two terms, $\left(1-I_{H}\right) \varepsilon_{H} \mathrm{~d} I_{H}$ and $I_{L} \varepsilon_{L} \mathrm{~d} I_{L}$, allow for endogenous reallocation of domestic workers. This is what we refer to as the internal reallocation effect. Again, notice the contrast to the original task-based approach by Grossman and Rossi-Hansberg (2008), where only the former channel is captured. Thus Eq. (3.C.69) can be seen as the generalization of Grossman and Rossi-Hansberg (2008) regarding skill heterogeneity.

Next, utilize the previously derived Eqs. (3.C.81), (3.C.67) and (3.C.68) in the relative demand equations (3.C.63)-(3.C.65). Then, after manipulating, rearranging and taking logs, we obtain the general equilibrium closed solution for the three endogenous task margins $\tilde{I}_{O}, I_{L}$ and $I_{H}$ :

$$
\begin{aligned}
\ln \left(\frac{A_{L} N_{L}}{A_{M} N_{M}}\right)= & -\ln \beta_{L}\left(I_{L}\right)+\ln \left(I_{L}-\tilde{I}_{O}\right)-\ln \left(I_{H}-I_{L}\right) \\
\ln \left(\frac{\mathcal{B}}{A_{M} N_{M}}\right)+\frac{1}{\alpha} \ln \omega= & -\frac{1}{\alpha}\left(\ln \beta_{L}\left(I_{L}\right)-\ln \beta_{L}\left(\tilde{I}_{O}\right)\right. \\
& \left.-\ln \tilde{\beta}_{O}\left(\tilde{I}_{O}\right)\right)-\ln \left(I_{H}-I_{L}\right)+\frac{1-\alpha}{\alpha} \ln \tilde{\Omega}(\cdot) \\
\frac{A_{M} N_{M}}{A_{H} N_{H}}= & -\ln \beta_{H}\left(I_{H}\right)+\ln \left(I_{H}-I_{L}\right)-\ln \left(1-I_{H}\right) .
\end{aligned}
$$

This $3 \times 3$ system of equations (3.C.70) can be utilized to compute the implicit solutions for the endogenous task margins due to any exogenous changes that are captured on the right hand sides.

\section{C.3 Comparative statics: Easier offshoring and skill-task reallocation}

Taking the total differentiation of the system (3.C.70) yields

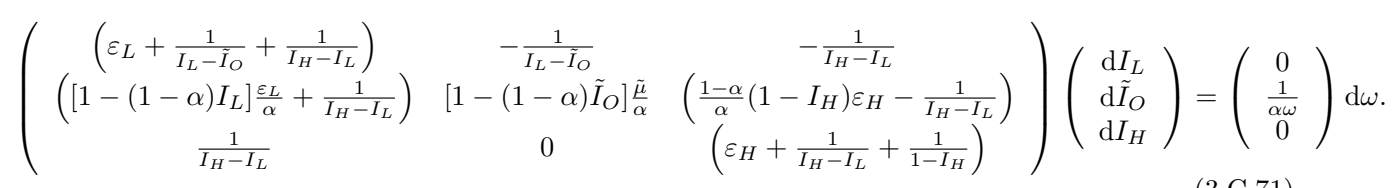


Computing the determinant of the $3 \times 3$ matrix yields

$$
\begin{aligned}
\Delta= & \frac{1}{\alpha\left(1-I_{H}\right)\left(I_{L}-\tilde{I}_{O}\right)\left(I_{H}-I_{L}\right)^{3}} \\
& {\left[\left(1-I_{L}+\left(1-I_{H}\right)\left(I_{H}-I_{L}\right) \varepsilon_{H}\right) \times\right.} \\
& \left(\tilde{\mu}\left(I_{H}-I_{L}\right)\left(\left(I_{L}-\tilde{I}_{O}\right)\left(I_{H}-I_{L}\right) \varepsilon_{L}+\left(I_{H}-\tilde{I}_{O}\right)\right)\left(1-(1-\alpha) \tilde{I}_{O}\right)\right. \\
& \left.+\left(I_{L}-\tilde{I}_{O}\right)\left(\left(I_{H}-I_{L}\right) \varepsilon_{L}\left(1-(1-\alpha) I_{L}\right)\right)\right)+\left(1+\left(1-I_{H}\right) \varepsilon_{H}\right)\left(I_{L}-\tilde{I}_{O}\right)\left(I_{H}-I_{L}\right) \alpha \\
& \left.+\left(1-I_{H}\right)\left(I_{L}-\tilde{I}_{O}\right)\left((1-\alpha)\left(I_{H}-I_{L}\right)\left(\left(1-I_{H}\right) \varepsilon_{H}-\tilde{\mu} \tilde{I}_{O}\right)+\tilde{\mu}\left(I_{H}-I_{L}\right)\right)\right]>0 .
\end{aligned}
$$

It is evident that all terms in the numerator are positive, implying that $\Delta$ is positive too. Given this result, the implicit solutions for the task margins due to easier offshoring are

$$
\begin{aligned}
\frac{\mathrm{d} I_{L}}{\mathrm{~d} \omega}= & \frac{1}{\Delta} \frac{\varepsilon_{H}+\frac{1}{1-I_{H}}+\frac{1}{I_{H}-I_{L}}}{\alpha\left(I_{H}-I_{L}\right) \omega}>0 \\
\frac{\mathrm{d} \tilde{I}_{O}}{\mathrm{~d} \omega}= & \frac{1}{\Delta} \frac{1}{\alpha\left(1-I_{H}\right)\left(I_{L}-\tilde{I}_{O}\right)\left(I_{H}-I_{L}\right)^{2} \omega} \\
& {\left[\left(\left(1-I_{H}\right)\left(I_{H}-I_{L}\right) \varepsilon_{H}+\left(1-I_{H}\right)+\left(I_{H}-I_{L}\right)\right)\left(\left(I_{L}-\tilde{I}_{O}\right)\left(I_{H}-I_{L}\right) \varepsilon_{L}+\left(I_{L}-\tilde{I}_{O}\right)+\left(I_{H}-I_{L}\right)\right)\right.} \\
& \left.+\left(1-I_{H}\right)\left(I_{L}-\tilde{I}_{O}\right)\right]>0 \\
\frac{\mathrm{d} I_{H}=}{\mathrm{d} \omega} & \frac{1}{\Delta} \frac{1}{\alpha\left(I_{H}-I_{L}\right)^{2} \omega}>0 .
\end{aligned}
$$

The following proposition summarizes the main results.

Proposition 3.8 (Offshoring low-skilled job-tasks and skill-task reallocation). If offshoring activities are permitted only to low-skill-intensive job tasks, then there exists an equilibrium threshold $\tilde{I}_{O}$ at which domestic firms allocate offshore workers to all tasks $i \in \tilde{I}_{O}$, where $0<\tilde{I}_{O}<I_{L}<I_{H}<1$. Moreover, easier offshoring $(\mathrm{d} \omega>0)$ induces an expansion of offshorable job-tasks as well as a job-task upgrading by low- and medium-skilled workers, i.e.

$$
\frac{\mathrm{d} \tilde{I}_{O}}{\mathrm{~d} \omega}>\frac{\mathrm{d} I_{L}}{\mathrm{~d} \omega}>\frac{\mathrm{d} I_{H}}{\mathrm{~d} \omega}>0 .
$$

Given these comparative statics, we can now investigate how offshoring affects both real and relative wages of domestic workers. We relegate the discussion on relative wages to the main text and focus here on real wage effects.

\section{C.4 Offshoring low-skilled job tasks and productivity effect}

To investigate the impact of offshoring low-skilled job tasks on real wages, recall the first-order conditions defining the optimal labor demand, and utilize the resource constraint conditions and the optimal demand 
condition for total employment, to obtain

$$
\begin{aligned}
w_{L} & =\frac{I_{L}-\tilde{I}_{O}}{N_{L}} P_{E} E=\frac{I_{L}-\tilde{I}_{O}}{N_{L}} P_{E}^{-\frac{1-\alpha}{\alpha}} \mathcal{B} \\
w_{M} & =\frac{I_{H}-I_{L}}{N_{M}} P_{E} E=\frac{I_{H}-I_{L}}{N_{M}} P_{E}^{-\frac{1-\alpha}{\alpha}} \mathcal{B} \\
w_{H} & =\frac{1-I_{H}}{N_{H}} P_{E} E=\frac{1-I_{H}}{N_{H}} P_{E}^{-\frac{1-\alpha}{\alpha}} \mathcal{B} .
\end{aligned}
$$

Next, combine the no-arbitrage condition (3.C.81) with the cost index of composite labor $P_{E}=\frac{w_{M}}{A_{M}} \tilde{\Omega}(\cdot)$ to substitute for $\frac{w_{M}}{A_{M}}$ in the inverse labor demand function of low- and high-skilled workers, respectively. Then, after further manipulation, we obtain

$$
\begin{aligned}
w_{L} & =\left(\frac{I_{L}-\tilde{I}_{O}}{N_{L}}\right)^{\alpha} \beta_{L}\left(I_{L}\right)^{1-\alpha} \tilde{\Omega}(\cdot)^{-(1-\alpha)} A_{L}^{1-\alpha} \mathcal{B}^{\alpha} \\
w_{M} & =\left(\frac{I_{H}-I_{L}}{N_{M}}\right)^{\alpha} \tilde{\Omega}(\cdot)^{-(1-\alpha)} A_{M}^{1-\alpha} \mathcal{B}^{\alpha} \\
w_{H} & =\left(\frac{1-I_{H}}{N_{H}}\right)^{\alpha} \beta_{H}\left(I_{H}\right)^{-(1-\alpha)} \tilde{\Omega}(\cdot)^{-(1-\alpha)} A_{H}^{1-\alpha} \mathcal{B}^{\alpha} .
\end{aligned}
$$

Taking logs and totally differentiating w.r.t. offshoring friction, the wage effect can be decomposed into the following terms:

$$
\begin{aligned}
\frac{\mathrm{d} \ln w_{L}}{\mathrm{~d} \omega}= & \left.\left(\frac{\alpha}{I_{L}-\tilde{I}_{O}}-(1-\alpha) \varepsilon_{L}\right)\right) \frac{\mathrm{d} I_{L}}{\mathrm{~d} \omega}+(1-\alpha)\left(I_{L} \varepsilon_{L} \frac{\mathrm{d} I_{L}}{\mathrm{~d} \omega}-\left(1-I_{H}\right) \varepsilon_{H} \frac{\mathrm{d} I_{H}}{\mathrm{~d} \omega}\right) \\
& +\left((1-\alpha) \tilde{I}_{O} \tilde{\mu}-\frac{\alpha}{I_{L}-\tilde{I}_{O}}\right) \frac{\mathrm{d} \tilde{I}_{O}}{\mathrm{~d} \omega} \\
\frac{\mathrm{d} \ln w_{M}}{\mathrm{~d} \omega}= & \left(\frac{\alpha}{I_{H}-I_{L}}-(1-\alpha)\left(1-I_{H}\right) \varepsilon_{H}\right) \frac{\mathrm{d} I_{H}}{\mathrm{~d} \omega}+\left((1-\alpha) I_{L} \varepsilon_{L}-\frac{\alpha}{I_{H}-I_{L}}\right) \frac{\mathrm{d} I_{L}}{\mathrm{~d} \omega} \\
& +(1-\alpha) \tilde{I}_{O} \tilde{\mu} \frac{\mathrm{d} \frac{\tilde{I}_{O}}{\mathrm{~d} \omega}}{\mathrm{d} \ln w_{H}} \\
\mathrm{~d} \omega & \left((1-\alpha) I_{H} \varepsilon_{H}-\frac{\alpha}{1-I_{H}}\right) \frac{\mathrm{d} I_{H}}{\mathrm{~d} \omega}+(1-\alpha)\left(I_{L} \varepsilon_{L} \frac{\mathrm{d} I_{L}}{\mathrm{~d} \omega}+\tilde{I}_{O} \tilde{\mu} \frac{\left.\mathrm{d} \frac{\tilde{I}_{O}}{\mathrm{~d} \omega}\right) .}{}\right.
\end{aligned}
$$

The last term, $(1-\alpha) \tilde{I}_{O} \tilde{\mu}$, in all three Eqs. (3.C.73)-(3.C.75) captures the aforementioned productivity effect. Notice that in Eq. (3.C.73) this productivity effect is interacting with the direct displacement effect of low-skilled workers, $\frac{\alpha}{I_{L}-\tilde{I}_{O}}$. However, comparing our results with that of Grossman and Rossi-Hansberg (2008), the important difference becomes evident. Next to the direct offshoring-induced productivity effect, there are two additional forces: the first and second terms in Eqs. (3.C.73)-(3.C.75) capture the internal reallocation effect. The extent of this internal reallocation effect depends on the substitutability between low- and medium-skilled workers as well as between medium- and high-skilled workers, which are captured by the relative task productivity parameters (semi-elasticities) $\varepsilon_{L}$ and $\varepsilon_{H}$, respectively. The following proposition summarizes the sufficient conditions.

Proposition 3.9 (Offshoring low-skilled job tasks and productivity effect). Given the impact of easier offshoring on the task reallocation derived in Proposition 3.8, an offshoring-induced cost-efficiency effect raises unambiguously the real wage of all skill groups for the sufficient conditions

1. $\varepsilon_{L} I_{L}>\varepsilon_{H}\left(1-I_{H}\right)$,

2. $\frac{\alpha}{1-\alpha} \frac{1}{I_{L}-\tilde{I}_{O}}>\varepsilon_{L}>\frac{\alpha}{1-\alpha} \frac{1}{I_{L}\left(I_{H}-I_{L}\right)}$, for $\tilde{I}_{O}>I_{L}\left[1-\left(I_{H}-I_{L}\right)\right]$ 
3. $\frac{\alpha}{1-\alpha} \frac{1}{\left(1-I_{H}\right)\left(I_{H}-I_{L}\right)}>\varepsilon_{H}>\frac{\alpha}{1-\alpha} \frac{1}{I_{H}\left(1-I_{H}\right)}$

4.

$\tilde{\mu}>\frac{\alpha}{1-\alpha} \frac{1}{\tilde{I}_{O}\left(I_{L}-\tilde{I}_{O}\right)}$.

Proof. The sufficient condition in Part 1. follows from the second term in Eq. (3.C.73) and the comparative static results $\frac{\mathrm{d} I_{L}}{\mathrm{~d} \omega}$ and $\frac{\mathrm{d} I_{H}}{\mathrm{~d} \omega}$ in Proposition 3.8. It states that for sufficient low substitutability between low- and medium-skilled workers compared to substitutability between medium- and high-skilled workers in the neighborhood of $I_{L}$ and $I_{H}$, respectively, offshoring-induced internal reallocation of workers is limited. If this sufficient condition holds, then the second term in Eq. (3.C.73) is unambiguously positive. In Part 2., the lower boundary of $\varepsilon_{L}$ is computed from the second term in Eq. (3.C.74), while the upper boundary follows from the first term in Eq. (3.C.73). Thus these conditions determine the sufficient range of values of substitutability between low- and medium-skilled workers. Notice however the qualifying necessary condition in this case that requires a sufficiently large range of offshoring. If this necessary condition does not hold, than the lower boundary in Part 2. becomes binding, implying that the first term in Eq. (3.C.73) will have a negative sign. However, it is worth noting that, even in this case, the real wage of low-skilled workers might increase if the two other positive terms in Eq. (3.C.73) are sufficiently strong. In Part 3., the lower boundary is computed from the first term in Eq. (3.C.75), while the upper boundary follows from the first term in Eq. (3.C.74). Finally, in Part 4., the sufficient condition following from the last term in Eq. (3.C.73) requires a sufficiently low substitutability between medium-skilled and offshore workers.

For these jointly sufficient conditions, the offshoring-induced labor supply effect on wages, characterized by the internal reallocation of domestic workers, is dominated by the overall rise in total domestic employment induced by the cost-efficiency effect due to lower offshoring frictions. This feature extends the models of Grossman and Rossi-Hansberg (2008) and Acemoglu and Autor (2011).

\section{C.5 Offshoring high-skill-intensive domestic job tasks}

As put forward in Blinder (2009b), many high-skill-intensive occupations might be affected by offshoring. Our framework can also be easily extended to account for this possible future development of globalization. To get an idea, we sketch the main properties followed by a graphical illustration.

In a similar vein, one can derive conditions characterizing the task allocation between offshore and highskilled workers. Considering the example of the quadratic function from above, the convenient property of the U-shaped task productivity schedule can be seen. In this case, the rise in international competition on domestic high-skill-intensive job-tasks can be described by an improvement in task productivity of offshore workers. Formally, we state

$$
\hat{\zeta}(i)=\exp \left[g_{M}\left(i-\hat{g}_{O}\right)^{2}\right]
$$

where now $\hat{g}_{O}>g_{O}>\tilde{g}_{O}>0$. Thus the U-shaped curve moves rightwards along the unit interval. Figure 3.7 provides a graphical illustration.

Similar to the case of offshoring low-skilled job tasks, the task assignment between offshore and highskilled workers will lead to unbundling of a fraction of high-skilled job tasks. This implies again that high-skilled workers are relatively cheaper in producing job tasks in the neighborhoods of $I_{H}$ and 1 , while offshore workers are cheaper somewhere in the middle. Formally, the assignment problem can be written as

$$
c_{O}(i) \lesseqgtr c_{H}(i)
$$

or equivalently

$$
\frac{\tau w_{O}}{A_{O}} \frac{1}{a_{O}(i)} \lesseqgtr \frac{w_{H}}{A_{H}} \frac{1}{a_{H}(i)}, \quad \forall i \in\left(I_{H}, 1\right) .
$$

Now, by a simple transformation, i.e. multiplying both sides in Eq. (3.C.76) by $a_{M}(i)$, we obtain the task productivity schedules, $\beta_{H}(i)=\frac{a_{M}(i)}{a_{H}(i)}$ and $\hat{\zeta}(i)=\frac{a_{M}(i)}{a_{O}(i)}$, with the same properties defined by Assumptions 1 and 2. Again the structure of the model is preserved. Now the necessary conditions for 
Figure 3.7: Offshoring high-skilled job tasks and skill-task allocation

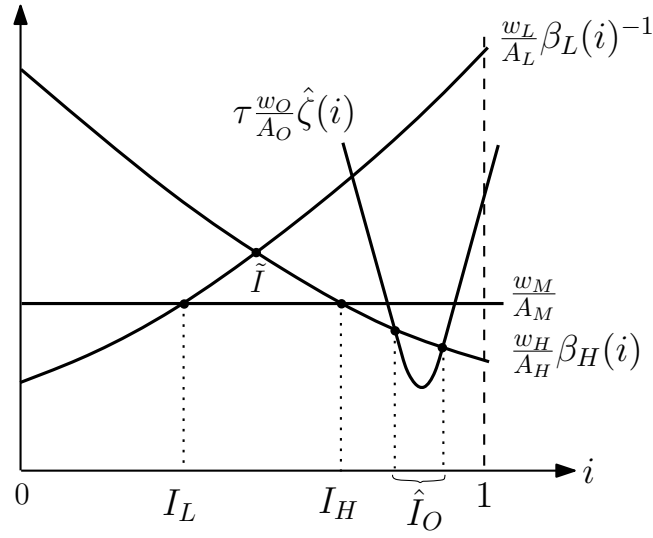

offshoring to be permitted to a subrange of high-skilled job tasks are

$$
\frac{A_{H}}{w_{H}} \beta_{H}(\ddot{I})^{-1} \hat{\zeta}(\ddot{I})<\omega<\frac{A_{H}}{w_{H}} \beta_{H}\left(I_{H}\right)^{-1} \hat{\zeta}\left(I_{H}\right), \quad \text { and } \quad I_{H}<\left.\hat{I}\right|_{\frac{\partial \ln \hat{\zeta}(\cdot)}{\partial i}=0}<1,
$$

implying that the offshoring friction is not too large (i.e. low values of $\omega=\frac{A_{O}}{\tau w_{O}}$ ), defined at the tangent point $\dot{I}$ between the productivity schedules $\left|\frac{\partial \beta_{H}(\ddot{I})}{\partial \ddot{I}}\right|=\left|\frac{\partial \tilde{\zeta}(\ddot{I})}{\partial \ddot{I}}\right|$, such that offshoring is permitted, nor too small, so that high-skilled workers do not lose their comparative advantage in competing with mediumskilled workers, defined by the task margin $I_{H}$. Then, given the assumptions and properties in (3.C.77), it follows from (3.C.76) that there must exist two cut-off margins defining the range of offshored highskill-intensive job tasks $\left(\hat{I}_{O}\right)$. By means of simple positive monotone transformation, this no-arbitrage condition is given by

$$
\frac{\hat{\beta}_{O}\left(\hat{I}_{O}\right)}{\omega}=\frac{w_{H}}{A_{H}}
$$

where $\hat{\beta}_{O}(\cdot)$ denotes the positive monotone transformation of relative task productivity schedules $\frac{\hat{\zeta}(\cdot)}{\beta_{H}(\cdot)}$ at the new task margins, $\hat{I}_{1}$ and $\hat{I}_{2}$, in terms of the offshoring range $\hat{I}_{O}$.

Notice again that the no-arbitrage conditions defining the domestic allocation of tasks to skills are preserved by Lemma 3.1. Thus the allocation of tasks across the different types of labor is as follows: low-skilled workers perform all tasks $i \in I_{L}$, medium-skilled workers perform all tasks $i \in\left(I_{H}-I_{L}\right)$, high-skilled workers perform all tasks $i \in\left(1-I_{H}-\hat{I}_{O}\right)$, and offshore workers perform all tasks $i \in \hat{I}_{O}$; see Figure 3.6 for these equilibrium allocations.

\section{C.6 Offshoring and domestic income distribution effect}

For the computation of the relative wage effects of offshoring, discussed in the main text, we need to adjust the relative labor demand between high- and medium-skilled workers for the range of offshoring $\hat{I}_{O}$. This follows from the new resource constraint for high-skilled workers and from the cost-minimized first-order conditions, from which the relative medium-skilled labor demand functions can be derived

$$
\begin{aligned}
\frac{w_{L}}{w_{M}} & =\frac{N_{M}}{N_{L}} \frac{I_{L}}{I_{H}-I_{L}} \\
\frac{w_{M}}{w_{H}} & =\frac{N_{M}}{N_{H}} \frac{I_{H}-I_{L}}{1-I_{H}-\hat{I}_{O}} .
\end{aligned}
$$


Recall the no-arbitrage conditions (3.2) and (3.3)

$$
\begin{aligned}
\frac{w_{L}}{w_{M}} & =\frac{A_{L}}{A_{M}} \beta_{L}\left(I_{L}\right) \\
\frac{w_{M}}{w_{H}} & =\frac{A_{M}}{A_{H}} \beta_{H}\left(I_{H}\right) .
\end{aligned}
$$

This set of Eqs. (3.C.79), (3.C.80) and (3.C.81) can be utilized to assess the impact of a marginal decline of offshoring friction on the relative domestic wages. For an intuitive and graphical discussion we refer the reader to discussion in section 3.5.2 in the main text. 

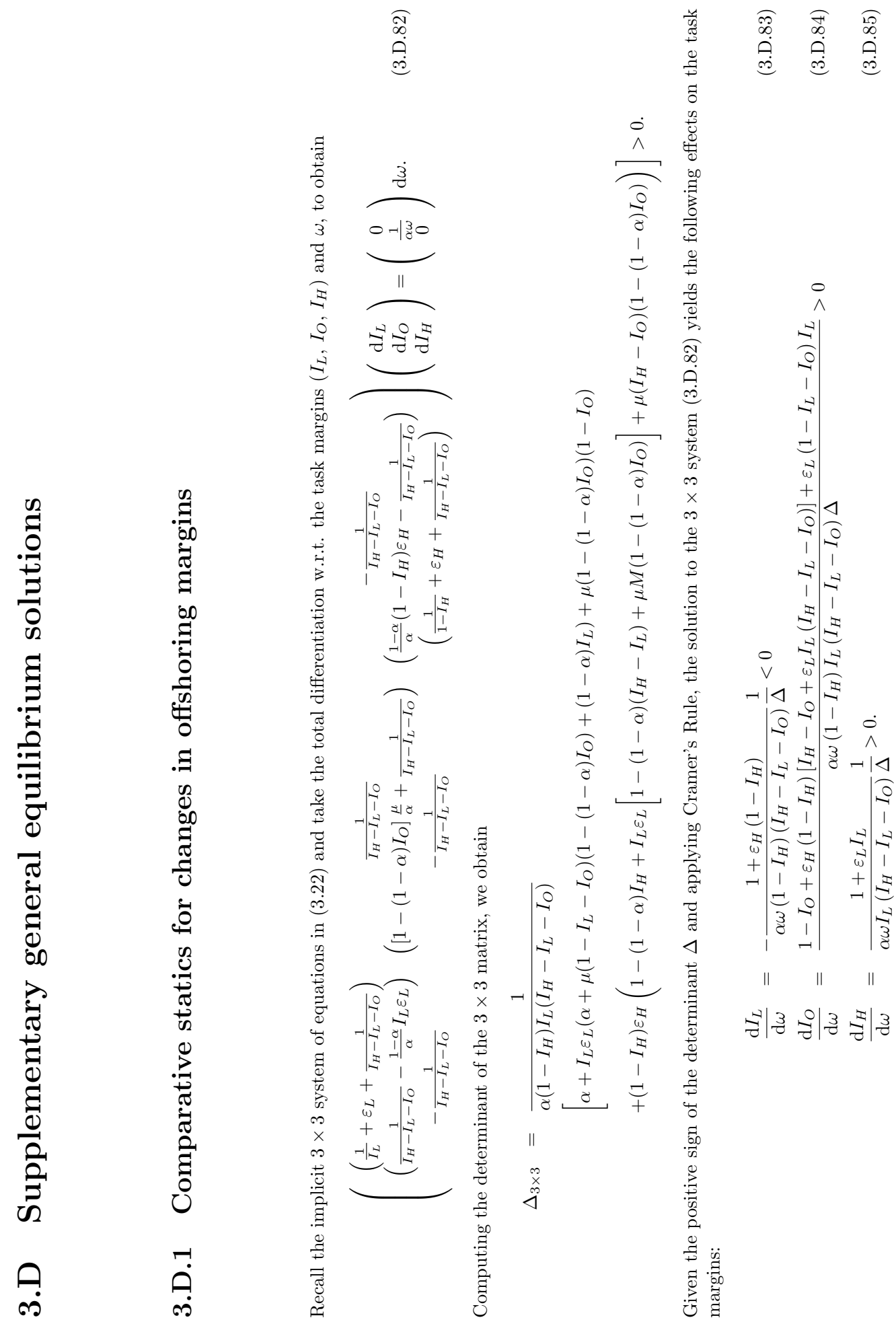
$\overparen{0}$
$\infty$
$\dot{0}$
$\dot{9}$

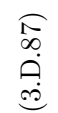

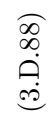
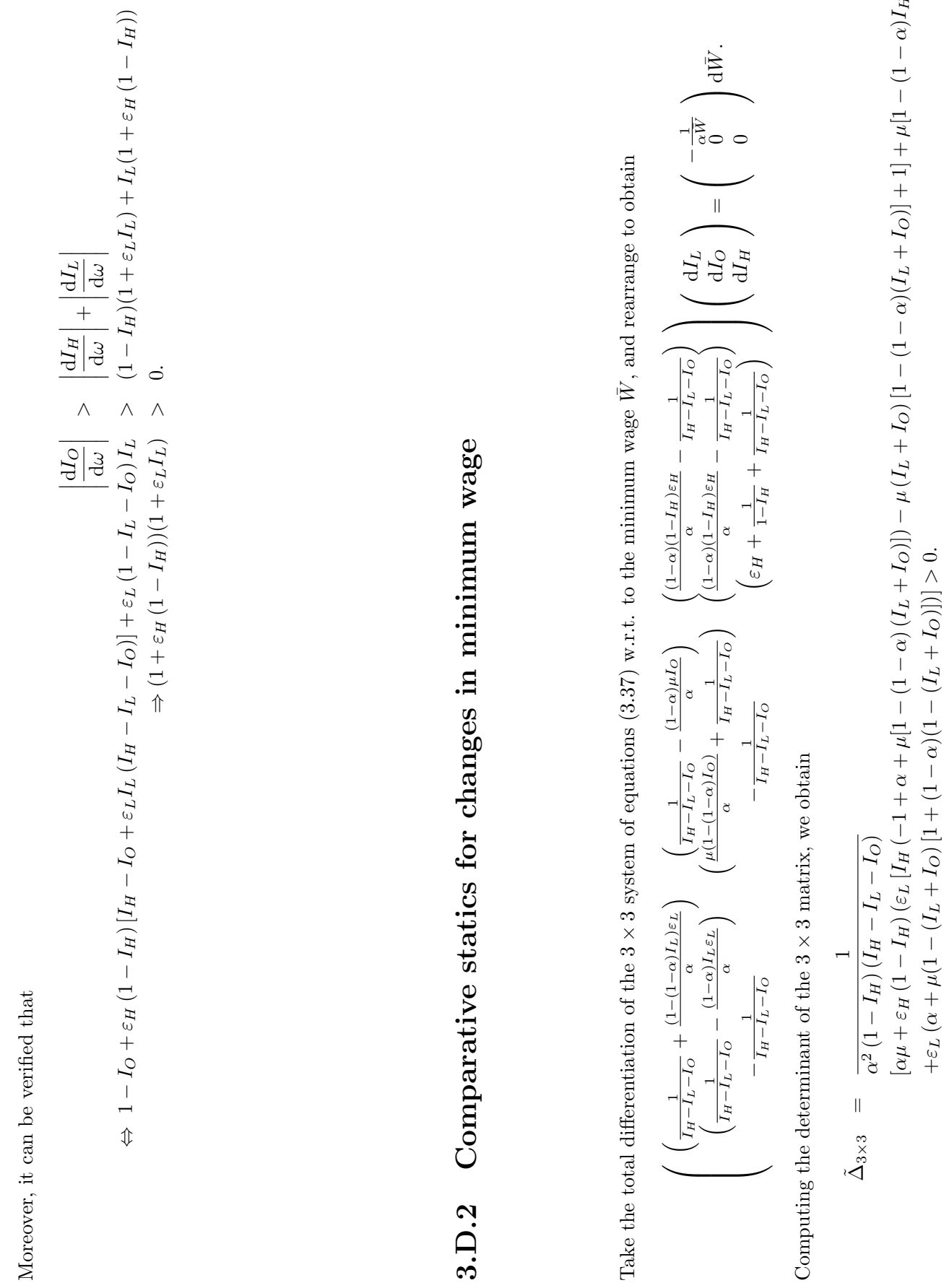


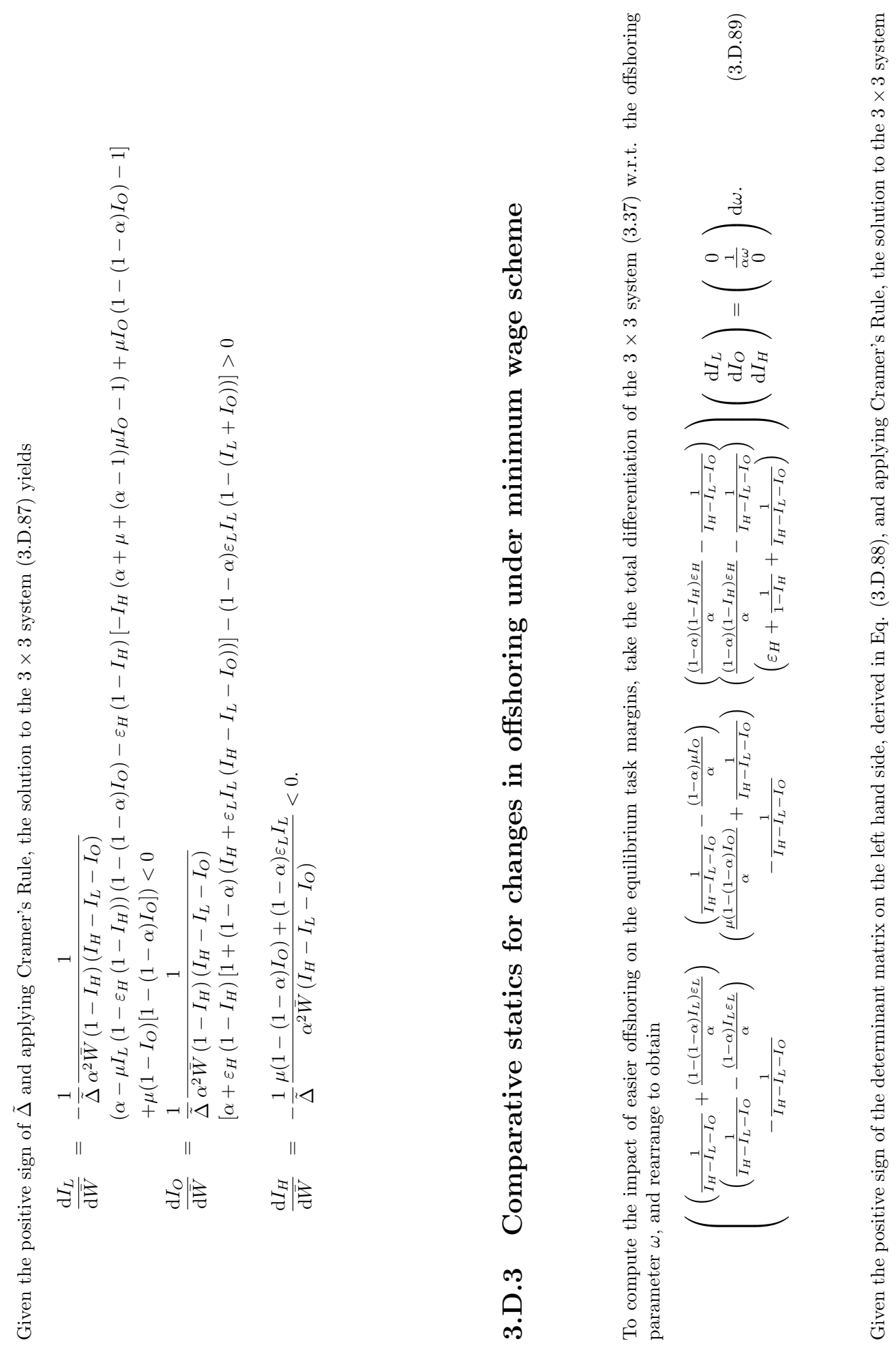


3.D.4 Comparative statics for changes in offshoring under endogenous low-skilled labor supply

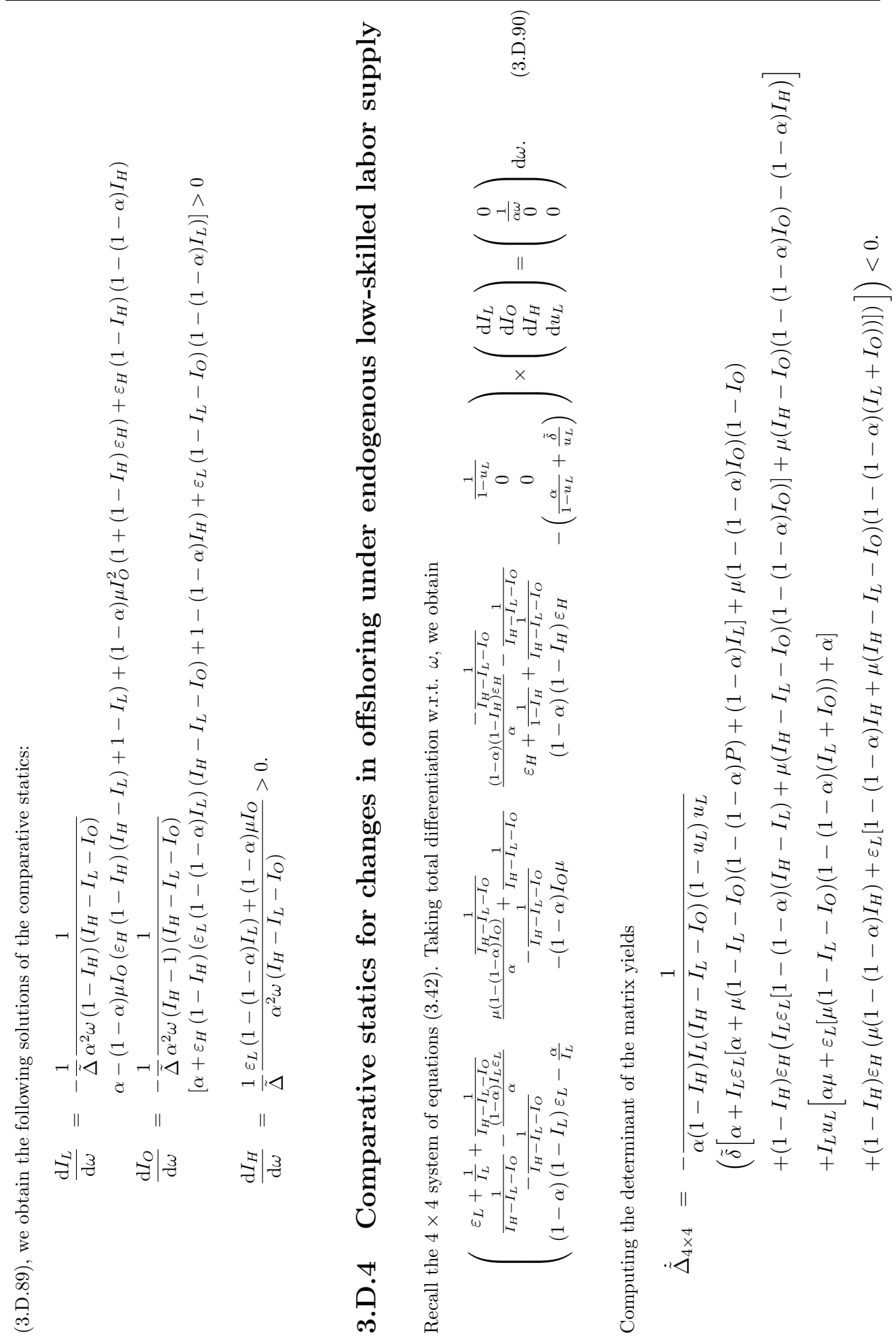



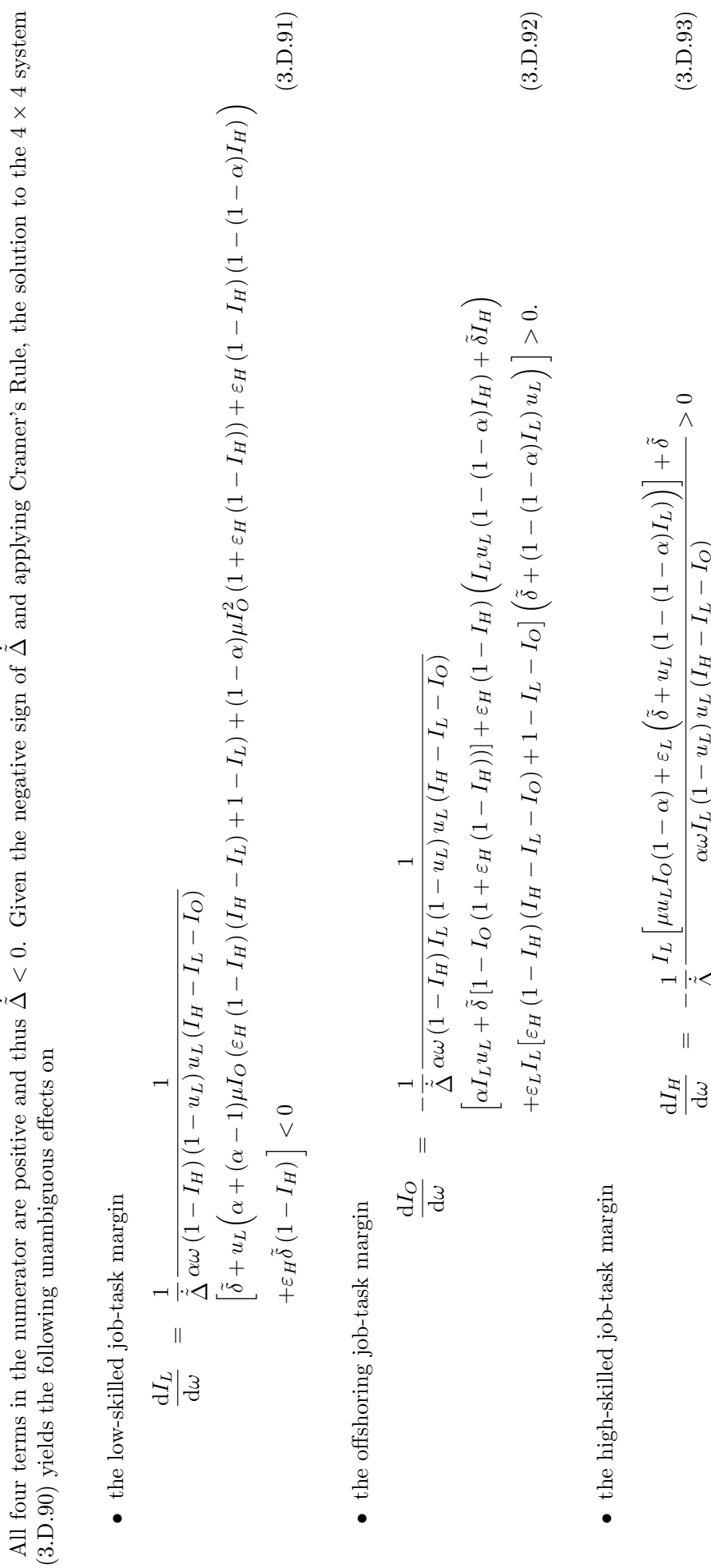

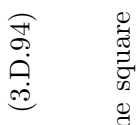

-

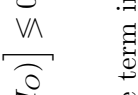

$\rightarrow$

$\begin{array}{ll}\frac{1}{2} & 0 \\ \frac{3}{3} & 0 \\ 1 & 0\end{array}$

$\stackrel{1}{=} \quad \uparrow$
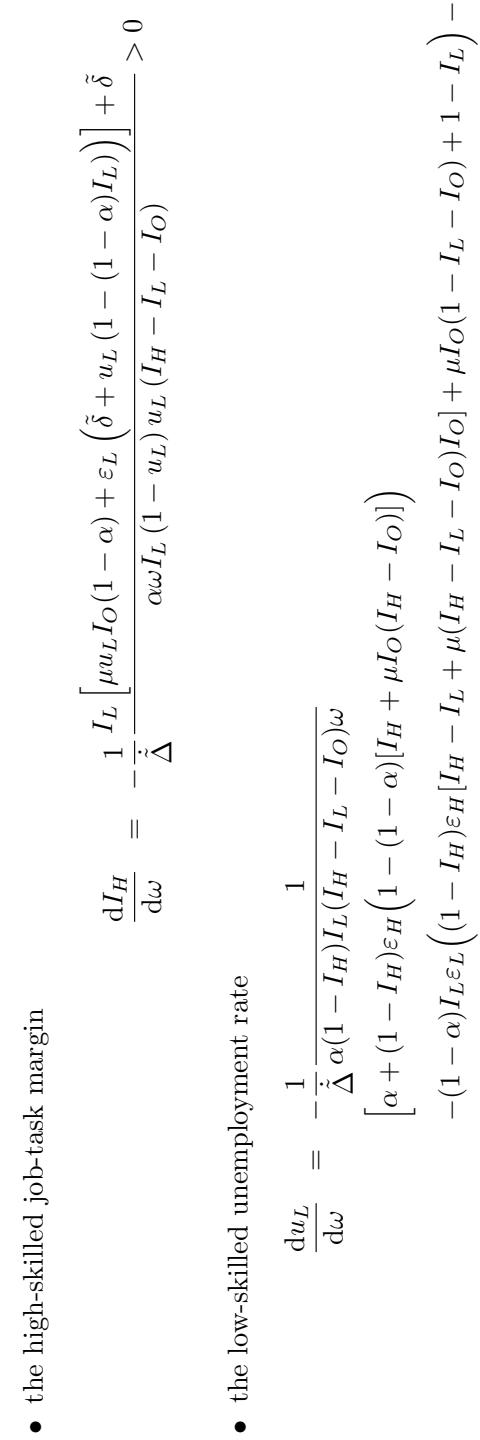

$\stackrel{-1}{-1}$

苟

$\frac{9}{0}$

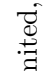

$\forall \quad:$

80 
3.D.4 Comparative statics for changes in offshoring under endogenous low-skilled labor supply

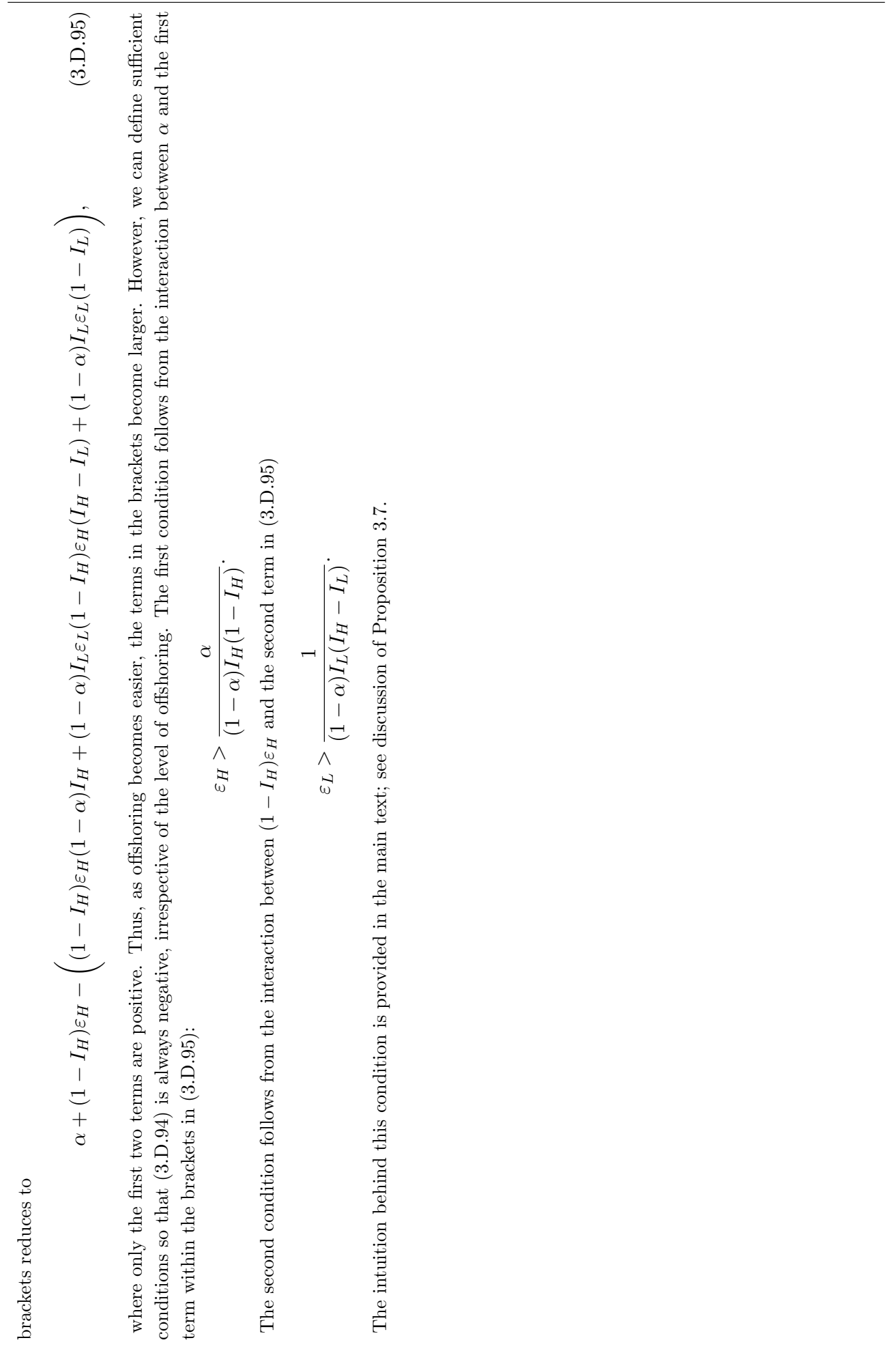




\section{Immigration, Technology Adoption and Wage Inequality $^{1}$}

\subsection{Introduction}

In many advanced countries, immigration has substantially increased the foreign-born population share, accompanied by considerable changes in their skill structure over the last decades. For example, many European Union member states have experienced a considerable shift in the skill structure of immigrants from low-towards medium- and high-skilled attainments (cf. Muysken et al., 2015). Consequently, immigration has a substantial impact on the skill mix of the workforce in the host country. Concerns regarding its labor market implication have considerably shaped both the public and academic discourse. In this paper, I exploit the potential channels by which immigrationinduced changes in the skill structure affect the domestic wage structure. In doing so, I develop a model that allows for a richer structure of the goods market, characterized by monopolistic competition, firm heterogeneity, and endogenous technology adoption, next to skill heterogeneity. As I elaborate below, these features are substantial to improve our understanding of adjustment mechanism behind the changes in the wage structure.

What does the empirical evidence tell us regarding the relationship between skill mix changes and potential adjustment mechanisms? One set of studies emphasizes the role of endogenous technology changes, the so-called directed technical change (cf. Acemoglu, 1998, 2002b). The idea is that an increase in the endowment of skilled workers (including those by immigration) induces a faster growth of skill-complement technologies, which in turn leads to a higher skill premium. This feature is captured in this paper through increasing returns to scale and the endogenous adjustment of the mass of firms to changes

\footnotetext{
${ }^{1}$ I am grateful for valuable comments and discussions to Joan Muysken and Thomas Ziesemer. I would also like to thank participants at TASK III - the $3^{\text {rd }}$ International BIBB/IAB/ZEW conference 2014 in Nuremberg, and at the annual AIEL conference 2015 in Cagliari.
} 
in the skill mix, indicating changes at the intensive margin. Related to this view, it has become a widespread consensus that skill-biased technological changes (SBTC), in particular unskilled labor-saving technological changes, has importantly contributed to the pervasive wage inequality in the 1980s, especially observed in the US (cf. Lemieux, 2008). The puzzling U-shaped wage trends after 1990 have been attributed to advances in

Figure 4.1: Changes in real male wages in the US by percentile

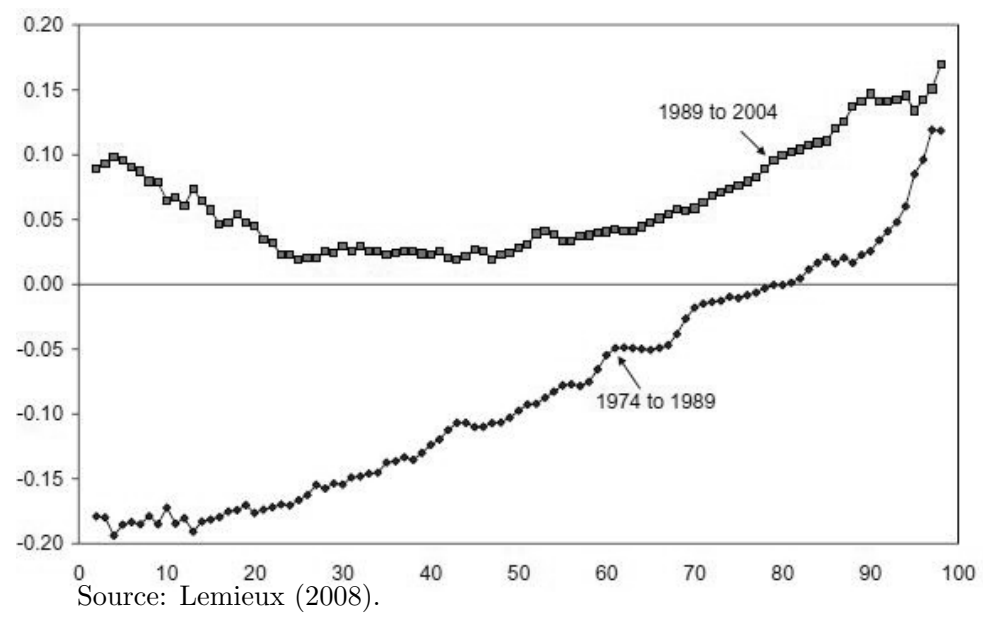

information communication technology (ICT), substituting mostly routine-intensive job tasks that are concentrated in the middle of the skill distribution and easily codifiable (cf. Acemoglu and Autor, 2011; Autor and Dorn, 2013; Autor et al., 2003) - also referred to as the computerization/routinization phenomenon. ${ }^{2}$ Figure 4.1 depicts these contrasting trends in the male wage structure that occurred at different time periods for the US. In this paper, I argue that a supply shock of medium-skilled workers may partly explain recent polarization trends also, due to a price effect for technology modes that complement low-skilled and high-skilled workers. I show that the key determinant behind this effect is the market power of monopolists.

Another set of studies provide a more competing view by highlighting the implications of changes in the skill endowment on endogenous technology adoption behavior of firms (Beaudry et al., 2010; Lewis, 2011). For example, Lewis (2011), provides evidence that the low adoption rate of automation machinery in manufacturing plants between 1988 and 1993 was associated with immigration-induced increase of low-skilled workers. Beaudry et al. (2010), using U.S. metropolitan area-level panel data, explore empirically the link between personal computer (PC) adoption and skill attainment. They find a positive relationship, where areas with an initially high relative supply of skill have experienced an increase in the skill premium when PC was introduced. However, both studies show that over time the relation between skill supply and its return dissipates since "investments in automation induced by immigration reduce the effect immigration has on wages" (Lewis, 2011, p. 1063), and thus balances the initial supply-driven wage effects.

\footnotetext{
${ }^{2}$ See Lemieux (2008) for a detailed survey of the literature regarding alternative explanations, such as changes in wage-setting institutions, regarding the rise in income inequality in many advanced countries.
} 
This channel denotes the important aspects of firm heterogeneity, explaining the asymmetry of firms in terms of productivity which has taken the center-stage in the new trade theory (cf. Melitz, 2003). In this paper, this feature is also captured but instead of a random selection, firms choose endogenously between various technology modes according to their comparative advantages (cf. Acemoglu and Zilibotti, 2001; Yeaple, 2005). I will refer to this channel as changes at the extensive margin.

Thus, the objective of this paper is to jointly investigate how changes at the extensive and intensive margin are determined and how they interact with each other. In doing so, I develop a model in which a continuum of industries (final goods) is combined to produce an aggregate consumption good. Each final good, in turn, can be produced by three different technology modes, where the extent of technology adoption is endogenous and reflects principles of comparative advantage, as in Acemoglu and Zilibotti (2001); Yeaple (2005). ${ }^{3}$ Each technology mode utilizes a distinct composite of intermediate goods, which are, in turn, produced under monopolistic competition, as in Krugman (1979). Furthermore, labor is the sole production factor in the intermediate goods market, implying that the labor market is heterogeneous and consists of three skill groups, each complement to one specific technology mode. In this setup, the equilibrium technology adoption is characterized by two endogenous margins, dividing the economy into three endogenous sets of sectors, each consisting of a continuum of industries.

This model uncovers several novel predictions regarding distributional effects of immigration-induced changes in the skill structure. First, I show that changes in the domestic wage structure can be decomposed into two key channels: i) a labor supply effect, and ii) technology adoption effect. Second, the interaction between these two channels is importantly determined by the interplay between two additional forces. On the one hand, it depends on the relation between consumer preferences (i.e. goods demand elasticity) and the market power of monopolists (i.e. elasticity of substitution between intermediate goods). On the other hand, it depends on the degree of adoption between the different technology modes (the extensive margin) which may mitigate the direct labor supply effect. The key implication of the model is that changes at the extensive margin (captured by the degree of technology adoption) countervail the direct supply effect. At the extreme case of easy adoption (i.e. perfect substitutability between technology modes) the two channels are balanced, so that changes in the wage structure become insensitive to labor supply shocks, reconciling the empirical evidence regarding the neutral long-run labor market effects of immigration.

Moreover, considering a more realistic range of parameter values, the analysis provides additional insights that reconcile the empirical observations regarding labor supply shocks and changes in the wage structure. For a sufficient degree of imperfect technology adoption, an immigration-induced increase of high-skilled workers induces changes in the wage structure similar to a "skill-biased" technical change, leading to a monotonic increase in the wage gap across the skill groups, whenever the demand elasticity is higher than the market power of monopolists. This conforms what Acemoglu (2002b) gives as explanation for why the relative demand for skill-complements goods has outpaced the

\footnotetext{
${ }^{3}$ The structure of this model is similar to the Ricardian model with a continuum of goods as in Dornbusch et al. (1977), except that now the equilibrium margins characterize the specialization pattern of the domestic industry, instead of between countries.
} 
skill supply over the last decades. These results uncover a new channel and highlight the importance of firm heterogeneity and endogenous technology adoption in analyzing labor market impacts of immigration. Particularly, these features help substantially to gain new insights regarding the underlying mechanism and the determinants of the adjustment process to immigration-induced changes in the domestic skill structure.

The paper is organized as follows. The next section briefly reviews the existing studies and relates this to the contributions of this paper. In section 4.3, the setup of the theoretical framework is presented. The characteristics of the equilibrium are discussed in section ??. The assessment of changes in the optimal technology adoption margins are presented in section 4.5, followed by the comparative statics of immigration-induced changes in the skill structure on the domestic wage structure in section 4.6. Finally, concluding remarks are provided in section 4.7 .

\subsection{Literature review}

There exist by now a vast number of studies that have examined various mechanisms by which immigration-induced changes in the skill mix affect native workers. Guided by the canonical nested CES-approach, with various extensions regarding capital-skill complementarity and labor market frictions, a large body of these studies provide empirical evidence that highlights the importance of complementarity and substitutability between immigrants and natives, translating mechanically the impact of immigration on the skill mix into an impact on wages - through the elasticity of substitution parameters. ${ }^{4}$

However, it is widely accepted that the effect of immigration on host country's labor market goes beyond this convenient mechanical relationship. ${ }^{5}$ Recent empirical studies, including Dustmann and Glitz (2012) for Germany, González and Ortega (2011) for Spain and Lewis (2003) for the US, have found strong evidence that much of the response to immigration-induced changes in the skill mix occurs within industries (or within firms), through changes in the skill intensity. They find that wages are relatively insensitive to labor supply shocks. These findings support the view "that immigration shocks induce changes in production technology at the industry level" (González and Ortega, 2011, p. $68)$.

Recent theoretical contributions have emphasized the adjustment channel through changes in the output mix (Felbermayr and Kohler, 2006, 2007; Iranzo and Peri, 2009; Muysken et al., 2015). According to these studies, an unbalanced immigration flow impacts the domestic wage structure through changes in output price, inducing shifts in the output structure. For instance, Muysken et al. (2015) show that the impact of labor supply shock on domestic wage structure depends importantly on the interaction between consumer preferences (i.e. the demand elasticity) and production technology (i.e. the elasticity of substitution between skill groups). ${ }^{6}$ In the context of the East-West

\footnotetext{
${ }^{4}$ Important contributions, among many others, are Borjas (2003); Borjas et al. (2011); Brücker et al. (2014); Brücker and Jahn (2011); Card (2009); D'Amuri et al. (2010); Manacorda et al. (2011); Ottaviano and Peri (2008, 2012); Peri (2011).

${ }^{5}$ See Lewis (2013) for a thorough survey of the related literature.

${ }^{6}$ Moreover, they allow for two types of labor market frictions, low-skill unemployment and skill-
} 
European integration, Iranzo and Peri (2009) discuss the gains from immigration and trade. They show that overall trade and immigration generate beneficial welfare effects for both regions, low-skilled workers in the West gain unambiguously in terms of real wages, while the real wage effect for high-skilled workers depends on the relative magnitude between the forces described by Muysken et al. (2015), i.e. the interaction between the elasticity of substitution between intermediates and the goods demand elasticity.

However, these models often rely on the standard assumptions of representative agents and ex-ante fixed production technologies. This paper augments these studies by using an alternative and richer structure of the goods market that allows for firm heterogeneity and endogenous choice of technique (Acemoglu and Zilibotti, 2001; Yeaple, 2005). The key implication of this set of models is that it allows to capture a broader impact of changes in the skill structure on the economy, as both the optimal choice of technology, the composition and the number of firms using different production techniques depend on the skill structure. Thus, the response of producers regarding the adoption of various production techniques to immigration-induced changes in the skill mix "may mediate immigration's ultimate labor market impact" (Lewis, 2013, p. 10). As I discuss below, the change in the domestic wage structure due to immigration-induced shifts in the skill mix is importantly affected by the degree of technology adoption. This is the novel contribution of this article.

\subsection{Theoretical framework}

I consider an economy that is characterized by an aggregated consumption good, which consists of a continuum of final goods. The final goods market is perfectly competitive, where final good producers are homogeneous ex ante. Following a widely used approach in the literature (cf. Acemoglu and Autor, 2011; Acemoglu and Zilibotti, 2001; Yeaple, 2005), I allow for ex post heterogeneity by introducing endogenous sorting into different technology modes based on comparative advantage differences, where firms self-elect endogenously into the most cost-efficient production techniques. ${ }^{7}$ The production of a final good requires the adoption of a distinct technology mode and the utilization of a complement composite of intermediate goods. Finally, monopolistically competitive firms produce an intermediate variety by utilizing labor as the sole factor.

To get a better idea, Figure 4.2 illustrates the structure of the framework, which will be discussed in detail in the next section. It is worth mentioning the crucial differences to Yeaple (2005): Instead of allocating different skill groups over the continuum interval into the different technologies, as in Yeaple (2005), intermediate goods producer determine the technology cut-off points. The latter allows to account for the elasticity of substitutability between intermediate goods. As discussed below, the direction of the distributional effect

downgrading of medium-skilled workers, and discuss the importance of labor market institutions for labor market outcomes of low-skilled workers. A shift from a rigid to a flexible unemployment benefit scheme regime induces a complementarity effect between low-skilled unemployment rate and the skill downgrading rate of medium-skilled workers, attenuating potential crowding-out effects of low-skilled workers.

${ }^{7}$ Notice the difference to the firm heterogeneity à la Melitz (2003), which allows for ex ante productivity heterogeneity. According to this view, heterogeneity is characterized by a random draw. 
due to immigration-induced changes in the skill mix is importantly determined by the size of this elasticity.

Figure 4.2: Structure of model

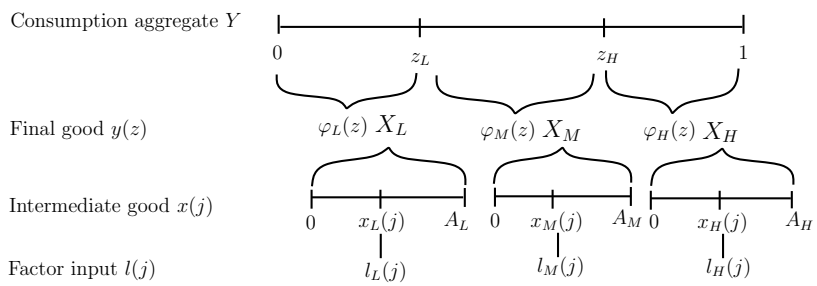

\subsubsection{Aggregate consumption good, final goods, and technology adoption}

As in Acemoglu and Zilibotti (2001), the aggregate consumption good $Y$ is produced by combining a continuum of final goods, $y(z)$, in a constant elasticity of substitution (CES) aggregate over the interval $[0,1]$. Each final good variety $z$ can be produced in different technology modes. More precisely, there exist three different modes: low $\left(\varphi_{L}\right)$, medium $\left(\varphi_{M}\right)$ or high $\left(\varphi_{H}\right)$. Similar to Yeaple (2005), the productivity schedule of each technology mode $\varphi(z)$ exhibits the following properties:

Assumption 4 (Technology schedule).

$$
\varphi_{L}(0)=\varphi_{M}(0)=\varphi_{H}(0)=1,
$$

and

$$
0<\frac{\mathrm{d} \ln \varphi_{L}(z)}{\mathrm{d} z}<\frac{\mathrm{d} \ln \varphi_{M}(z)}{\mathrm{d} z}<\frac{\mathrm{d} \ln \varphi_{H}(z)}{\mathrm{d} z} \text {, for all } z \in[0,1] \text {. }
$$

Therefore, the final goods are ordered so that the higher indexed goods are most productive with the high-quality technology.

Given the properties of the productivity schedules imposed by Assumption 4, there exist two thresholds, denoted by $z_{L}$ and $z_{H}$, which determine the cost-efficient allocation of the three technology modes in the economy. More precisely, we get that the aggregate consumption good $Y$ is produced by the following constant elasticity of substitution (CES) function

$$
Y=\left[\int_{0}^{z_{L}} y_{L}(z)^{\frac{\sigma-1}{\sigma}} \mathrm{d} z+\int_{z_{L}}^{z_{H}} y_{M}(z)^{\frac{\sigma-1}{\sigma}} \mathrm{d} z+\int_{z_{H}}^{1} y_{H}(z)^{\frac{\sigma-1}{\sigma}} \mathrm{d} z\right]^{\frac{\sigma}{\sigma-1}}
$$

where $\sigma \geq 1$ denotes the elasticity of substitution between the final goods. Each final good is produced by combining a composite of intermediate goods, $X_{k}$ with the complement 
technology $\varphi_{k}(z)$

$$
y(z)= \begin{cases}\varphi_{L}(z) X_{L} & \text { for all } z \in\left[0, z_{L}\right] \\ \varphi_{M}(z) X_{M} & \text { for all } z \in\left(z_{L}, z_{H}\right) \\ \varphi_{H}(z) X_{H} & \text { for all } z \in\left[z_{H}, 1\right]\end{cases}
$$

Then, given the production function (5.1), profit maximization yields the optimal demand for any final good $z$,

$$
y_{k}(z)=Y p_{k}(z)^{-\sigma}, \text { for } k=\{L, M, H\}
$$

From (5.1) and (4.3) the corresponding price index of $Y$ is given by

$$
1=\left[\int_{0}^{z_{L}} p_{L}(z)^{1-\sigma} \mathrm{d} z+\int_{z_{L}}^{z_{H}} p_{M}(z)^{1-\sigma} \mathrm{d} z+\int_{z_{H}}^{1} p_{H}(z)^{1-\sigma} \mathrm{d} z\right]^{\frac{1}{1-\sigma}}
$$

where the price of the consumption aggregate is normalized to unity, i.e. $P_{Y}=1$, so that all goods and factor prices are in real terms.

The perfectly competitive nature of final goods market implies that every producer takes its own price, $p(z)$, and the marginal cost of $X_{k}, \zeta_{k}$, as given when optimizing its production cost. Moreover, in equilibrium any producer utilizing technology mode $k$ must make zero profits, i.e. $p_{k}(z) y_{k}(z)=\zeta_{k} X_{k}$. Using this observation and making use of Eq. (4.2), then the final goods price can be written as

$$
p(z)= \begin{cases}P_{L} \varphi_{L}(z)^{-1} & \text { for all } z \in\left[0, z_{L}\right] \\ P_{M} \varphi_{M}(z)^{-1} & \text { for all } z \in\left(z_{L}, z_{H}\right) \\ P_{H} \varphi_{H}(z)^{-1} & \text { for all } z \in\left[z_{H}, 1\right]\end{cases}
$$

where $P_{k}$ denotes the effective price index of final goods, i.e. the price per productive technology $k$, which will be defined below.

As illustrated in Figure 4.3, the price-technology schedule implies that the price of final goods adjust to productivity differences. It follows that in equilibrium the cut-off margins are defined by two cost-efficient conditions, summarized by the following lemma.

Lemma 4.1 (Efficient Technology Allocation). In equilibrium, the final good price adjusts endogenously for the productivity differences in technology. For marginal final goods $z_{L}$ and $z_{H}$ and the price indexes $P_{L}, P_{M}$ and $P_{H}$ of the respective technology mode, it follows

$$
\begin{aligned}
& \frac{P_{L}}{P_{M}}=\Lambda_{L}\left(z_{L}\right) \equiv \frac{\varphi_{L}\left(z_{L}\right)}{\varphi_{M}\left(z_{L}\right)}, \\
& \frac{P_{M}}{P_{H}}=\Lambda_{H}\left(z_{H}\right) \equiv \frac{\varphi_{M}\left(z_{H}\right)}{\varphi_{H}\left(z_{H}\right)}
\end{aligned}
$$

and

$$
0<z_{L}<z_{H}<1, \quad \text { for } \quad \frac{P_{L}}{P_{M}} \in\left(\Lambda_{L}\left(z_{H}\right), \Lambda_{L}(0)\right) \text { and } \frac{P_{M}}{P_{H}} \in\left(\Lambda_{H}(1), \Lambda_{H}\left(z_{L}\right)\right) .
$$

Proof. See Appendix 4.A.1. 
Figure 4.3: Final goods price and technology productivity schedules

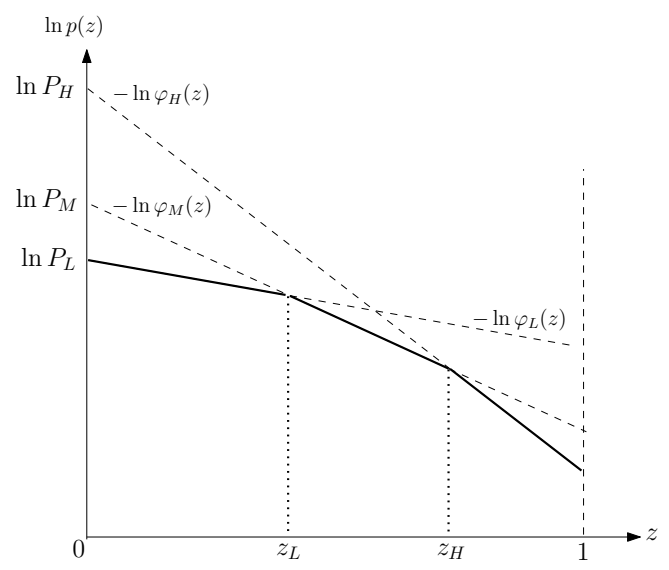

In Figure 4.3 the solid kinked curve can be interpreted as the equilibrium price-technology frontier (PTF).

\subsubsection{Intermediate goods market}

In contrast to the final goods market, the intermediate goods market is characterized by monopolistic competition, where each intermediates, in turn, is manufactured by workers. In addition, each variety has to incur a fixed output, denoted by $f_{k}$, that must be produced but can not be sold. As in Yeaple (2005), it is assumed that these fixed product development outputs are measured in terms of the numeraire good and the size of this fixed output depends on the technology mode adopted, where $f_{H}>f_{M}>f_{L}$ is imposed. ${ }^{8}$

This specification of the fixed costs aims for the sake of tractability of the model. Alternative specification, such as measuring the fixed costs in terms of factor labor, would complicate considerably the labor market clearing condition.

The production technology of the composite good $X_{k}$ is represented by a Constant Elasticity of Substitution (CES) aggregator over a continuum of varieties, indexed by $j$ :

$$
X_{k}=\left(A_{k}^{\frac{\nu-1}{\epsilon}} \int_{0}^{A_{k}} x_{k}(j)^{\frac{\epsilon-1}{\epsilon}} \mathrm{d} j\right)^{\frac{\epsilon}{\epsilon-1}},
$$

where $x(j)$ denotes the amount of intermediate variety $j \in\left[0, A_{k}\right], \epsilon \geq 1$ captures the elasticity of substitution between varieties, and $A_{k}$ measures the mass of monopolists in technology mode $k$. The parameter $\nu$ determines the degree of external increasing returns to scales of the production process. For limiting values of $\nu=0$, the differentiated good $X$ is independent of $A$, cf. Egger et al. (2013). For $\nu=\epsilon-1$, the CES aggregator

\footnotetext{
${ }^{8}$ This specification aims for the sake of simplicity of measuring the fixed costs, simplifying substantially the derivation of the model equilibrium. Particularly, measuring the fixed costs in terms of factor labor would complicate considerably the labor market clearing condition, see Yeaple (2005) for a discussion.
} 
becomes becomes linear in $A$, as in Acemoglu et al. (2012). Here, I impose the special case where $\nu=1$, as in Iranzo and Peri (2009). In this case the production technology is characterized by external increasing returns to scale. ${ }^{9}$

Each intermediate is produced by a single monopolist which is characterized by a constant return to scale production technology with labor as the sole input. Given the wage rate $w_{k}$ in sector $k$, each monopolists employs $l_{k}$ units of labor, i.e. $x_{k}(j)=l_{k}(j)$.

\section{Demand for intermediate goods}

In any technology mode $k=\{L, M, H\}$, the optimal amount of intermediates is obtained by minimizing the cost, $\int_{0}^{A_{k}} p_{k}(j) x_{k}(j) \mathrm{d} j$ subject to $(5.7)$ which yields the following inverse demand function for intermediates (see Appendix 4.B.1 for the derivation):

$$
p_{k}(j)=\zeta_{k} X_{k}^{\frac{1}{\epsilon}} x_{k}(j)^{-\frac{1}{\epsilon}}, \forall k=\{L, M, H\}
$$

where $p_{k}(j)$ is the price of each intermediate variety produced with technology mode $k$. Combining (4.10) with (5.7) yields the marginal cost of composite intermediate goods

$$
\zeta_{k}=\left(\int_{0}^{A_{k}} p_{k}(j)^{1-\epsilon} \mathrm{d} j\right)^{\frac{1}{1-\epsilon}} .
$$

\section{Intermediate firm behavior}

Under the constant elastic demand function (4.10) of degree $\epsilon>1$ and the linear property of the production technology, the profit maximizing monopolist sets the price equal to a markup $\epsilon /(\epsilon-1)$ over the respective marginal cost, i.e.

$$
p_{k}(j)=\frac{\epsilon}{\epsilon-1} w_{k},
$$

where $w_{k}$ denotes the marginal cost of $k$-type labor.

Having defined the structure of the economy and the optimality conditions, the next section proceeds with the characteristics of the equilibrium.

\subsection{Characteristics of equilibrium}

This section discusses the model equilibrium by deriving optimal conditions characterizing firms behavior, labor market clearing, and the allocation of resources to technology modes. Moreover, the analysis relies on the standard symmetric equilibrium assumption in the intermediate goods market, implying that the varieties of the differentiated good $X_{k}$

\footnotetext{
${ }^{9}$ However, it is worth mentioning that for an empirically plausible value of the elasticity of substitution $\epsilon=2$ both limiting cases $\nu=1$ and $\nu=\epsilon-1$ generate equivalent analytical results (cf. Ottaviano and Peri, 2012).
} 
employ the same amount of factor labor $l_{k}(j)=l_{k}$, are sold at the same price $p_{k}(j)=p_{k}$ and are produced in equal amount $x_{k}(j)=x_{k}$, for all $k=\{L, M, H\}$.

Let the total amount of labor used by the intermediate-goods firms be denoted by $n_{k}$, i.e. $\int_{0}^{A_{k}} l_{k} \mathrm{~d}=n_{k}$, and the total (exogenously given) endowments of labor (i.e. over the range of final goods produced with technology mode $k$ ) by $N_{L}, N_{M}$, and $N_{H}$, i.e.

$$
\int_{z \in \mathcal{S}_{k}} n_{k} \mathrm{~d} z=N_{k},
$$

where $\mathcal{S}_{L}=\left\{0, z_{L}\right\}, \mathcal{S}_{M}=\left\{z_{L}, z_{H}\right\}$, and $\mathcal{S}_{H}=\left\{z_{H}, 1\right\}$, such that

$$
\begin{aligned}
l_{L} & =\frac{N_{L}}{z_{L} A_{L}}, \\
l_{M} & =\frac{N_{M}}{\left(z_{H}-z_{L}\right) A_{M}}, \\
l_{H} & =\frac{N_{H}}{\left(1-z_{H}\right) A_{H}} .
\end{aligned}
$$

Recall the assumption that each monopolists bears a fixed product development output (cf. Yeaple, 2005). This implies that the effective unit labor employed by each intermediate firm in technology mode $k$ is given by $f_{k}+x_{k}$, so that the fixed costs are given by $w_{k} f_{k}$. Free entry ensures that each differentiated-good firm makes zero profits so that $\frac{1}{\epsilon} p_{k} x_{k}=w_{k} f_{k}$. Then, the quantity for each variety is defined by

$$
x_{k}=(\epsilon-1) f_{k}, \quad \forall \quad k=\{L, M, H\} .
$$

From Eq. (4.11) it follows that the marginal cost of the differentiated good reduces to $\zeta_{k}=A_{k}^{\frac{1}{1-\epsilon}} p_{k}$. Now, using the monopolistic pricing behavior, Eq. (5.10), yields

$$
\zeta_{k}=\frac{\epsilon}{\epsilon-1} A_{k}^{\frac{1}{1-\epsilon}} w_{k}, \quad \forall k=\{L, M, H\}
$$

Substituting the free entry condition (5.14) into the composite intermediate goods function, Eq. (5.7), and using Eqs. (4.2) and (4.5) and the resource constraints (5.17), (5.19), and (5.18), then from the the budget constraint for any final good producer $z$,

$$
p(z) y(z)=w_{k} \int_{0}^{A_{k}} l_{k}(j) \mathrm{d} j,
$$

the mass of monopolists in technology mode $k$ can be pinned down to the skill endowment, fixed costs, the range of final goods, and the degree of substitutability between final goods 
(see Appendix 5.B.1 for details of the formal derivation).

$$
\begin{aligned}
A_{L} & =\frac{N_{L}}{\epsilon f_{L} z_{L}}, \\
A_{M} & =\frac{N_{M}}{\epsilon f_{M}\left(z_{H}-z_{L}\right)}, \\
A_{H} & =\frac{N_{H}}{\epsilon f_{H}\left(1-z_{H}\right)} .
\end{aligned}
$$

Before proceeding, it is worth noticing the following properties of the equilibrium measure of the mass of monopolists. From Eqs. (4.18)-(4.20), it is readily seen that changes in the endowment of labor induce an increase in the respective the mass monopolists in the complementary technology mode, given $z_{L}$ and $z_{H}$. Intuitively, the increase in the skill endowment of type $k$ employed by monopolists in sector $k$ induces a market size effect, raising the profitability of producing intermediate goods $x_{k}$ and thus leading to an increase in the mass of monopolists hiring those type of workers. This effect captures the idea of aforementioned directed technical change at the intensive margin. However, as elaborated below, the technology modes margins, $z_{L}$ and $z_{H}$, will respond endogenously to skill mix changes, generating a "spill-over" effect for the other technology modes. This effect captures changes at the extensive margin. The implication of these forces for the wage structure and their interaction with each other are elaborated below.

Next, utilizing the labor market clearing conditions (5.17), (5.18) and (5.19), the output per monopolist using technology modes $k=\{L, M, H\}$ can be written, respectively, as

$$
x_{L}=\frac{n_{L}}{A_{L}}=\frac{N_{L}}{z_{L} A_{L}}, \quad x_{M}=\frac{n_{M}}{A_{M}}=\frac{N_{M}}{\left(z_{H}-z_{L}\right) A_{M}}, \quad x_{H}=\frac{n_{H}}{A_{H}}=\frac{N_{H}}{\left(1-z_{H}\right) A_{H}} .
$$

Substituting (4.21) into the composite intermediate goods function (5.7) for the respective technology mode, we obtain

$$
\begin{aligned}
X_{L} & =A_{L}^{\frac{1}{\epsilon-1}} \frac{N_{L}}{z_{L}}, \\
X_{M} & =A_{M}^{\frac{1}{\epsilon-1}} \frac{N_{M}}{z_{H}-z_{L}}, \\
X_{H} & =A_{H}^{\frac{1}{\epsilon-1}} \frac{N_{H}}{1-z_{H}} .
\end{aligned}
$$

Utilizing (5.21) into Eq. (4.2), then the production function of final goods can be rewritten as

$$
y(z)= \begin{cases}\varphi_{L}(z) X_{L}=\varphi_{L}(z) A_{L}^{\frac{1}{\epsilon-1}} \frac{N_{L}}{z_{L}}, & \text { for all } z \in\left[0, z_{L}\right], \\ \varphi_{M}(z) X_{M}=\varphi_{M}(z) A_{M}^{\frac{1}{\epsilon-1}} \frac{N_{M}}{z_{H}-z_{L}}, & \text { for all } z \in\left(z_{L}, z_{H}\right), \\ \varphi_{H}(z) X_{H}=\varphi_{H}(z) A_{H}^{\frac{1}{\epsilon-1}} \frac{N_{H}}{1-z_{H}}, & \text { for all } z \in\left[z_{H}, 1\right],\end{cases}
$$

Finally, we derive the equilibrium aggregate levels of output and expenditure for each 
technology mode $k$. Let the total cost using technology $k$ be

$$
P_{k} Y_{k}=\int_{z \in \mathcal{S}_{k}} p(z) y(z) \mathrm{d} z
$$

where $Y_{k}$ denotes the aggregate level of output of final goods using technology mode $k$. Now, utilizing Eqs. (4.5) and (4.23) in the previously derived equation and manipulating slightly, yields the aggregate level of output per technology mode $k$ (see Appendix 4.B.3 for the formal derivation)

$$
\begin{aligned}
Y_{L} & =A_{L}^{\frac{1}{\epsilon-1}} N_{L}, \\
Y_{M} & =A_{M}^{\frac{1}{\epsilon-1}} N_{M}, \\
Y_{H} & =A_{H}^{\frac{1}{\epsilon-1}} N_{H} .
\end{aligned}
$$

The demand for final goods, Eq.(4.3), indicates that $p(z) y(z)=Y p(z)^{1-\sigma}$ for all $z \in$ $[0,1]$. Furthermore, Eqs. (4.5) and (4.2) imply that $p_{L}\left(z_{L}\right) y_{L}\left(z_{L}\right) / Y=p\left(z_{L}\right)^{1-\sigma}=$ $p_{M}\left(z_{L}\right) y_{M}\left(z_{L}\right) / Y$ and $p_{M}\left(z_{H}\right) y_{M}\left(z_{H}\right) / Y=p\left(z_{H}\right)^{1-\sigma}=p_{H}\left(z_{H}\right) y_{H}\left(z_{H}\right) / Y$. That is, the expenditure share on final goods, produced under different technology modes, must be equal at the equilibrium margins, $z_{L}$ and $z_{H}$. Utilizing Eqs. (4.5) and (4.23), then yield

$$
\frac{P_{L} Y_{L}}{z_{L}}=\frac{P_{M} Y_{M}}{z_{H}-z_{L}}, \quad \text { and } \quad \frac{P_{M} Y_{M}}{z_{H}-z_{L}}=\frac{P_{H} Y_{H}}{1-z_{H}}
$$

Finally, we solve for the equilibrium expressions of the margins, $z_{L}$ and $z_{H}$. Substituting Eqs. (4.24) for the respective aggregate output $Y_{L}, Y_{M}$, and $Y_{H}$ in Eqs. (4.25) and using the equilibrium values of the mass of monopolists, (4.18), (4.19), and (4.20), and taking ratios, we obtain two equations characterizing as follows

$$
\begin{aligned}
& \frac{P_{L}}{P_{M}}=\left(\frac{f_{L}}{f_{M}}\right)^{\frac{1}{(\epsilon-1)}}\left(\frac{N_{M}}{N_{L}}\right)^{\frac{\epsilon}{(\epsilon-1)}}\left(\frac{z_{L}}{z_{H}-z_{L}}\right)^{\frac{\epsilon}{(\epsilon-1)}}, \\
& \frac{P_{M}}{P_{H}}=\left(\frac{f_{M}}{f_{H}}\right)^{\frac{1}{(\epsilon-1)}}\left(\frac{N_{H}}{N_{M}}\right)^{\frac{\epsilon}{(\epsilon-1)}}\left(\frac{z_{H}-z_{L}}{1-z_{H}}\right)^{\frac{\epsilon}{(\epsilon-1)}} .
\end{aligned}
$$

Thus, Eqs. (5.28) and (5.29) reflect the aggregate relative prices of technology mode $M$. Next, combine (5.28) and (5.29) with the efficient allocation of technology modes, Eqs. (5.3) and (5.4), derived in Lemma 4.1, respectively. Now, taking logs, manipulating and rearranging slightly, we obtain two implicit functions $\mathcal{F}_{L}(\cdot)$ and $\mathcal{F}_{H}(\cdot)$ that define the equilibrium technology margins $z_{L}$ and $z_{H}$ as functions of parameters and exogenous variables.

$$
\begin{aligned}
\mathcal{F}_{L}\left(z_{L}, z_{H}, f_{L}, f_{M}, N_{L}, N_{M}\right) \equiv & \frac{1}{\epsilon} \ln \left(\frac{f_{L}}{f_{M}}\right)+\ln \left(\frac{N_{M}}{N_{L}}\right)-\frac{(\epsilon-1)}{\epsilon} \ln \Lambda_{L}\left(z_{L}\right) \\
& -\ln \left(\frac{z_{H}-z_{L}}{z_{L}}\right)=0 \\
\mathcal{F}_{H}\left(z_{L}, z_{H}, f_{H}, f_{M}, N_{H}, N_{M}\right) \equiv & \frac{1}{\epsilon} \ln \left(\frac{f_{M}}{f_{H}}\right)+\ln \left(\frac{N_{H}}{N_{M}}\right)-\frac{(\epsilon-1)}{\epsilon} \ln \Lambda_{H}\left(z_{H}\right) \\
& -\ln \left(\frac{1-z_{H}}{z_{H}-z_{L}}\right)=0 .
\end{aligned}
$$


This implicit system of equations can be used to compute basic comparative statics of changes in factor endowment and fixed market entry costs and their implications for technology adoption.

\subsection{Immigration and changes in technology adoption margins}

Consider first the implication of immigration-induced changes in the medium-skill endowment on the equilibrium technology mode margins, $z_{L}$ and $z_{H}$. Total differentiation of the implicit Eqs. (5.30) and (5.31) yields

$$
\begin{aligned}
\left(\begin{array}{ll}
\frac{\partial \mathcal{F}_{L}}{\partial z_{L}} & \frac{\partial \mathcal{F}_{L}}{\partial z_{H}} \\
\frac{\partial \mathcal{F}_{H}}{\partial z_{L}} & \frac{\partial \mathcal{F}_{H}}{\partial z_{H}}
\end{array}\right)\left(\begin{array}{c}
\mathrm{d} z_{L} \\
\mathrm{~d} z_{H}
\end{array}\right)=\left(\begin{array}{c}
-\frac{\partial \mathcal{F}_{L}}{\partial N_{M}} \\
-\frac{\partial \mathcal{F}_{H}}{\partial N_{M}}
\end{array}\right) \times \mathrm{d} N_{M}, \\
\Leftrightarrow\left(\begin{array}{cc}
\frac{\epsilon-1}{\epsilon} \frac{\tilde{\varepsilon}_{L}}{z_{L}}+\frac{1}{z_{H_{H}-z_{L}}}+\frac{1}{z_{L}} & -\frac{1}{z_{H}-z_{L}} \\
-\frac{1}{z_{H}-z_{L}} & \frac{1}{\epsilon}
\end{array}\right)\left(\begin{array}{l}
\mathrm{d} z_{L} \\
\mathrm{~d} z_{H}
\end{array}\right)=\left(\begin{array}{c}
-\frac{1}{N_{M}} \\
\frac{1}{N_{M}}
\end{array}\right) \times \mathrm{d} N_{M},
\end{aligned}
$$

where $\tilde{\varepsilon}_{k} \equiv-\frac{\partial \Lambda_{k}\left(z_{k}\right)}{\partial z_{k}} \frac{z_{k}}{\Lambda_{k}\left(z_{k}\right)}>0$, for $k=\{L, H\}$ denotes the elasticity (in absolute value) of relative efficiency schedules of technology modes $k$ at the equilibrium margin $z_{k}$. The inspection of the matrix verifies that the Jacobi has a positive sign, i.e. $\mathcal{D}_{J}=\frac{\partial \mathcal{F}_{L}}{\partial z_{L}} \frac{\partial \mathcal{F}_{H}}{\partial z_{H}}-$ $\frac{\partial \mathcal{F}_{L}}{\partial z_{H}} \frac{\partial \mathcal{F}_{H}}{\partial z_{L}}>0$, and thus the solution to the above $2 \times 2$ system is unique. Then, applying Cramer's rule, we obtain

$$
\frac{\mathrm{d} z_{L}}{\mathrm{~d} N_{M}}=\frac{\mathcal{D}_{L}}{\mathcal{D}_{J}}=-\frac{1}{N_{M}} \frac{\frac{1}{1-z_{H}}+\frac{(\epsilon-1) \tilde{\varepsilon}_{H}}{\epsilon z_{H}}}{\mathcal{D}_{J}}<0,
$$

and

$$
\frac{\mathrm{d} z_{H}}{\mathrm{~d} N_{M}}=\frac{\mathcal{D}_{H}}{\mathcal{D}_{J}}=\frac{1}{N_{M}} \frac{\frac{\epsilon+(\epsilon-1) \tilde{\varepsilon}_{L}}{\epsilon z_{L}}}{\mathcal{D}_{J}}>0
$$

Thus, by implication the overall range of final goods produced with technology mode $M$ is increased

$$
\frac{\mathrm{d}\left(z_{H}-z_{L}\right)}{\mathrm{d} N_{M}}>0
$$

Figure 4.4 depicts the equilibrium technology margins and the results of the above comparative statics for any arbitrary comparative advantage schedules, $\Lambda_{L}$ and $\Lambda_{H}$. It can be easily verified that the relative technology demand condition (5.28) is increasing in $z_{L}$ from the origin, for given values of $z_{H}$, while Eq. (5.3) is decreasing in $z_{L}$ from above. Thus, there is a single crossing, characterizing the equilibrium technology margin $z_{L}$. This is denoted by point a in Figure 4.4. Similarly, from the relative technology demand condition (5.29) and the efficiency condition (5.4) one can show the single crossing between the two curves, as denoted by point b in Figure 4.4 . 
Figure 4.4: Medium-skill immigration and changes in the equilibrium technology margins

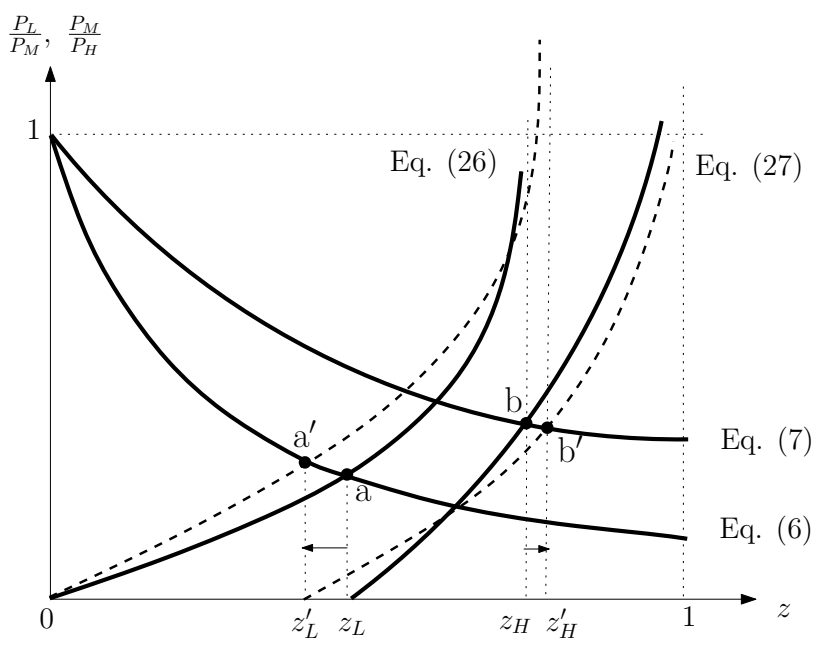

Now, changes in the composite factor labor $N_{M}$ (e.g. due to easier offshoring or immigration of medium-skilled workers) induce simultaneously an upward and downward shift of the curves Eq. (5.28) and Eq. (5.29), respectively. This leads to a decline and increase in equilibrium margins $z_{L}$ and $z_{H}$, respectively. After further adjustments in both curves - a slight rightward shift in Eq. (5.28) and a leftward shift in Eq. (5.29) - due to changes in the $z_{L}$ and $z_{H}$, the new equilibrium approaches eventually to points a ${ }^{\prime}$ and $\mathrm{b}^{\prime}$. A similar exercise can be performed to compute the comparative statics for changes in both fixed costs $\left(f_{k}\right)$ and labor endowments $N_{L}$ and $N_{H}$. The following proposition summarizes the main results.

Proposition 4.1 (Comparative statics of technology adoption for changes in fixed costs and labor endowment). Exogenous changes in the skill endowment and in fixed costs will affect the technology margins $z_{L}$ and $z_{H}$ in the following way:

- Immigration of high-skill workers and $H$-fixed cost

$$
\left\{\frac{\mathrm{d} z_{L}}{\mathrm{~d} \ln N_{H}}, \frac{\mathrm{d} z_{H}}{\mathrm{~d} \ln N_{H}}\right\}<0,
$$

- Immigration of medium-skilled workers and $M$-fixed cost

$$
\frac{\mathrm{d} z_{L}}{\mathrm{~d} \ln N_{M}}<0, \frac{\mathrm{d} z_{H}}{\mathrm{~d} \ln N_{M}}>0,
$$

- Immigration of low-skilled workers and L-fixed cost

$$
\left\{\frac{\mathrm{d} z_{L}}{\mathrm{~d} \ln N_{L}}, \frac{\mathrm{d} z_{H}}{\mathrm{~d} \ln N_{L}}\right\}>0 .
$$


Proof. A full analytical derivation is provided in Appendix 4.A.2.

Intuitively, an increase in the factor endowment $N_{k}$, results in an expansion of $X_{k}$ as a larger factor market allows for more varieties, i.e. the mass of monopolists $\left(A_{k}\right)$ increases. A larger factor market also implies a higher competition among workers of the same type and thus by the perfectly competitive nature of the labor markets, induces a decline in their wages. This, in turn, reduces the marginal cost of the differentiated good $\left(\zeta_{k}\right)$ and thus the overall price index of final goods $\left(P_{k}\right)$, making the utilization of technology $k$ more profitable compared to other types of technology, $k^{\prime} \neq k$. Consequently, more final good producers adopt technology $k$, and the economy becomes proportionately more specialized in technology mode $k$.

\subsection{Immigration and distributional effect}

Equipped with the comparative statics for technology adoption, the impact of immigration-induced changes in the skill endowment on the domestic wage structure can be analyzed. In equilibrium, the intermediate goods demand condition (4.10) and the monopolistic price-setting condition (5.10) imply

$$
\begin{aligned}
& \frac{\epsilon}{\epsilon-1} w_{L}=p_{L}=\zeta_{L} X_{L}^{1 / \epsilon} x_{L}^{-1 / \epsilon} \\
& \frac{\epsilon}{\epsilon-1} w_{M}=p_{M}=\zeta_{M} X_{M}^{1 / \epsilon} x_{M}^{-1 / \epsilon} \\
& \frac{\epsilon}{\epsilon-1} w_{H}=p_{H}=\zeta_{H} X_{H}^{1 / \epsilon} x_{H}^{-1 / \epsilon}
\end{aligned}
$$

To compute the changes in the domestic wage structure, take the ratio between low- and medium-skilled wages and between medium- and high-skilled wages to obtain

$$
\begin{aligned}
\frac{w_{L}}{w_{M}} & =\frac{\zeta_{L}}{\zeta_{M}}\left(\frac{X_{L}}{X_{M}}\right)^{1 / \epsilon}\left(\frac{x_{L}}{x_{M}}\right)^{-1 / \epsilon}, \\
\frac{w_{M}}{w_{H}} & =\frac{\zeta_{M}}{\zeta_{H}}\left(\frac{X_{M}}{X_{H}}\right)^{1 / \epsilon}\left(\frac{x_{M}}{x_{H}}\right)^{-1 / \epsilon} .
\end{aligned}
$$

Recall again that due to the perfectly competitive nature of the final goods market in equilibrium $P_{k}=\zeta_{k}$ for all $k=\{L, M, H\}$ must hold. Using this observation together with the equilibrium outcomes (4.21), (5.21), (4.24), (4.25), (5.28), and (5.29) in Eqs. (4.32) and (4.33), and taking logs, we obtain the following relative wage rate of mediumskilled workers

$$
\begin{aligned}
& \ln \left(\frac{w_{L}}{w_{M}}\right)=-\frac{\sigma-\epsilon}{(\epsilon-1) \sigma} \ln \left(\frac{z_{L}}{z_{H}-z_{L}}\right)+\frac{\sigma-\epsilon}{(\epsilon-1) \sigma}\left(\ln N_{L}-\ln N_{M}\right)+\ln \mathcal{K}_{L, M}( \\
& \ln \left(\frac{w_{M}}{w_{H}}\right)=-\frac{\sigma-\epsilon}{(\epsilon-1) \sigma} \ln \left(\frac{z_{H}-z_{L}}{1-z_{H}}\right)-\frac{\sigma-\epsilon}{(\epsilon-1) \sigma}\left(\ln N_{H}-\ln N_{M}\right)+\ln \mathcal{K}_{H, M}
\end{aligned}
$$

where $\mathcal{K}_{L, M}$ and $\mathcal{K}_{H, M}$ capture other constant variables, see Appendix 5.B.3 for the formal derivation. 
Thus, changes in the domestic wage structure can be decomposed into two key factors: i) a labor supply effect, $\ln N_{L}, \ln N_{H}$, and $\ln N_{M}$; ii) a technology adoption effect, $\ln \left(\frac{z_{L}}{z_{H}-z_{L}}\right)$ and $\ln \left(\frac{z_{H}-z_{L}}{1-z_{H}}\right)$. Moreover, an inspection of Eqs. (4.34) and (4.35) reveals immediately that the extent of the direct labor supply effect and the technology adoption effect depends crucially on the relative magnitude between the goods demand elasticity $(\sigma)$ and the market power of monopolists $(\epsilon)$. The intuition behind these two forces is the following. Immigration induces a market size effect and a price effect.

To get a better idea, consider the immigration of low-skill workers. In this case, firms employing low-skill workers experience a cost-efficiency enhancing, leading to higher output $\left(x_{L}\right)$ and profits $\left(\pi_{L}\right)$ and thus to an increase in the mass of those monopolists $\left(A_{L}\right)$. This effect is referred to as the direct market size effect. Consequently, the comparative advantage of adopting technology mode $L$ increases, leading to an increase in the relative price index of final goods produced with technology modes $M$ and $H$. This in turn induces firms in the final goods market to switch to production techniques complementing lowskill workers, leading to an increase in the cut-off margin $z_{L}$. This adjustment can be referred to as the indirect market size effect.

However, this latter effect raises the relative profitability of technology modes $M$ and $H$ compared to $L$, and thus counteracting the former direct effect. This can be easily verified from Eqs. (4.18), (4.19), and (4.20), where $A_{L}$ declines in $z_{L}$, while $A_{M}$ and to a larger extent $A_{H}$ rise as the respective range of final goods declines. This effect reflects the price effect associated with a biased shift in favor of technology modes $M$ and $H{ }^{10}$ As these direct and indirect scale effects work in opposite directions the relative strength between them is ambiguous, and will importantly depend on the relative magnitude between $\sigma$ and $\epsilon$, and the degree of technology adoption.

First, the degree of technology adoption, i.e. changes in the cut-off margins $z_{L}$ and $z_{H}$, affects the extent of immigration-induced changes in the wage structure. This magnitude is captured by the elasticity parameters $\tilde{\varepsilon}_{L} \equiv-\frac{\mathrm{d} \ln \Lambda_{L}\left(z_{L}\right)}{\mathrm{d} \ln z_{L}}$ and $\tilde{\varepsilon}_{H} \equiv-\frac{\mathrm{d} \ln \Lambda_{H}\left(z_{H}\right)}{\mathrm{d} \ln z_{H}}$ (measured in absolute terms) in the neighborhood of $z_{L}$ and $z_{H}$, respectively. Thus, whenever technology adoption in the neighborhood of $z_{L}$ becomes very easy, indicating very low values of $\tilde{\varepsilon}_{L}$, changes in the relative price indices $P_{L} / P_{M}$ become insensitive to marginal changes in the margin $z_{L}$ induced by low-skill immigration. In Figure 4.4 , this case is associated with a flatting of the curve Eq. 5.3, where in the limit $\tilde{\varepsilon}_{L} \rightarrow 0$ it becomes a horizontal line.

Thus, if $\tilde{\varepsilon}_{L}, \rightarrow 0$, the direct and indirect scale effects will be asymptotically fully balanced, so that the relative wage between low- and medium-skill stays asymptotically unchanged. However, the change in the relative wage structure at the upper part of the skill distribution, i.e. between medium- and high-skill workers, may still exist and is similarly affected by the degree of technology adoption at the margin $z_{H}$, captured by the elasticity parameter $\tilde{\varepsilon}_{H}$. These are novel features of the model, reconciling the aforementioned empirical findings regarding technology adoption and changes in the wage structure. More importantly, they highlight the underlying determinants behind the

\footnotetext{
${ }^{10} \mathrm{It}$ is also worth mentioning that the direct and indirect market size effects have a similar interpretation to the internal and external scale effects mentioned in Ethier (1982), but differ importantly in terms of their determinants and the context.
} 
adjustment mechanisms.

Moreover, as discussed below, for positive and finite degrees of technology adoption, i.e. $\left\{\tilde{\varepsilon}_{L}, \tilde{\varepsilon}_{H}\right\}>0$, changes in the wage structure are determined by the interaction between goods demand elasticity and the elasticity of substitution between intermediates. Thus, for $\sigma>\epsilon$, the goods demand effect will be the dominating force, inducing a favorable demand shift for goods exhibiting a relative decline in their prices, through variation in market size effects. Whenever $\epsilon=\sigma$, both the market size effect and the price effect are fully balanced and the overall wage structure remains unchanged. I discuss now these channels and their interaction in more detail.

\subsubsection{Low-skill and high-skill immigration and distributional effect}

In this section, I analyze the wage effects of immigration at the tails of the skill distribution. Although in many advanced countries the past pattern of international migration was often characterized by this bimodal skill distribution (cf. Felbermayr and Kohler, 2007), I will separately examine the effects of low-skill and high-skill immigration in order to provide clear analytical results. ${ }^{11}$

To compute the impact of low-skill immigration, take the total differentiation of Eqs. (4.34) and (4.35) with respect to $N_{L}$ to obtain

$$
\begin{aligned}
\frac{\mathrm{d} \ln \left(w_{L} / w_{M}\right)}{\mathrm{d} \ln N_{L}} & =\frac{\sigma-\epsilon}{(\epsilon-1) \sigma}\left[1-\Gamma_{L}\right], \\
\frac{\mathrm{d} \ln \left(w_{M} / w_{H}\right)}{\mathrm{d} \ln N_{L}} & =-\frac{\sigma-\epsilon}{(\epsilon-1) \sigma} \Delta_{L} .
\end{aligned}
$$

where $\Gamma_{L} \equiv \frac{\mathrm{d} \ln \left[z_{L} /\left(z_{H}-z_{L}\right)\right]}{\mathrm{d} \ln N_{L}}$ and $\Delta_{L} \equiv \frac{\mathrm{d} \ln \left[\left(z_{H}-z_{L}\right) /\left(1-z_{H}\right)\right]}{\mathrm{d} \ln N_{L}}$ denote the relative change in the range of technology modes due to low-skill immigration and by Proposition $4.1 \Gamma_{L}>0$ and $\Delta_{L}<0$.

Similarly, computing the impact of high-skill immigration, we obtain from Eqs. (4.34) and (4.35)

$$
\begin{aligned}
\frac{\mathrm{d} \ln \left(w_{L} / w_{M}\right)}{\mathrm{d} \ln N_{H}} & =-\frac{\sigma-\epsilon}{(\epsilon-1) \sigma} \Gamma_{H}, \\
\frac{\mathrm{d} \ln \left(w_{M} / w_{H}\right)}{\mathrm{d} \ln N_{H}} & =-\frac{\sigma-\epsilon}{(\epsilon-1) \sigma}\left[1+\Delta_{H}\right],
\end{aligned}
$$

where now $\Gamma_{H}$ and $\Delta_{H}$ denote the relative change in the range of technology modes due to low-skill immigration, and by Proposition $4.1, \Gamma_{H}>0$ and $\Delta_{H}<0$.

It is immediately evident that low-skill and high-skill immigration affects relative wages

\footnotetext{
${ }^{11}$ However, each scenarios may be considered to reflect specific moments in the past, e.g. the guest workers program in many European countries in the 1970s, reflecting low-skill immigration scenario; or the collapse of Soviet Union early 1990s which lead to an increase of immigration of scientists (e.g. mathematicians and physicists) to the US, reflecting high-skill immigration scenario.
} 
between low-skilled and medium-skilled workers and between medium-skilled and highskilled workers through the two channels, the labor supply and the technology adoption effects, respectively. However, the endogenous technology choice will affect the overall wage structure in the economy. As mentioned earlier, changes in the wage structure is determined by the interaction between goods demand elasticity and the market power of monopolists, and the extent final-good producers are able to adopt the skill-complement technology modes in the neighborhood of $z_{L}$ and $z_{H}$. The next proposition summarizes the main results.

Proposition 4.2 (Low-skill and High-skill Immigration and Changes in the Wage Structure). Immigration of low-skilled and high-skilled workers generates a market size effect, raising the competition among same skilled workers. It also enhances the comparative advantage of the technology mode and the mass of intermediate goods complementing the respective skill. For a sufficient positive and finite degree of technology adoption, i.e. $0<\left\{\tilde{\varepsilon}_{L}, \tilde{\varepsilon}_{H}\right\}<\infty$, immigration has the following impact on the wage structure

(i) Low-skilled immigration induces a wage effect similar to "unskill-biased" technical change, i.e. a rise in relative increase in the wage rate of low-skilled workers compared to medium-skilled workers, and a relative rise in the wage rate of medium-skill workers compared to high-skilled workers, whenever the goods demand elasticity is larger than the market power of monopolists (elasticity of substitution between intermediate goods), i.e. $\sigma>\epsilon$; the converse is true if $\sigma<\epsilon$.

(ii) High-skill immigration leads to a monotonic increase in the skill premium similar to the impact of "skill-biased" technical change, i.e. a relative increase in the wage rate of medium-skilled workers compared to low-skilled workers, and a relative rise in the wage rate of high-skill workers compared to medium-skilled workers,, whenever the goods demand elasticity is larger than the market power of monopolists (elasticity of substitution between intermediate goods), i.e. $\sigma>\epsilon$; the converse is true if $\sigma<\epsilon$.

Moreover, if in the neighborhood of technology margins $z_{L}$ and $z_{H}$

(iii) the degree of substitution between technology modes becomes extremely easy, i.e. $\left\{\tilde{\varepsilon}_{L}, \tilde{\varepsilon}_{H}\right\} \rightarrow 0$, then the domestic wage structure becomes asymptotically insensitive to immigration,

(iv) the degree of substitution between technology modes in the neighborhood of margins, $z_{L}$ and $z_{H}$, becomes extremely difficult, i.e. $\left\{\tilde{\varepsilon}_{L}, \tilde{\varepsilon}_{H}\right\} \rightarrow \infty$, then the relative wage between medium-skilled and high-skilled workers becomes asymptotically insensitive to low-skill immigration, while the relative wage between low-skilled and mediumskilled is insensitive to high-skill immigration.

Proof. See Appendix 4.A.3.

As discussed above, lower values of $\tilde{\varepsilon}_{L}\left(\tilde{\varepsilon}_{H}\right)$ indicate that for final good producer it is easy to substitute $(H-) L$-technology mode by $M$-technology mode in the neighborhood of $z_{L}$ $\left(z_{H}\right)$. Recalling Figure 4.4, in the limit of perfect substitutability, i.e. $\left\{\tilde{\varepsilon}_{L}, \tilde{\varepsilon}_{H}\right\} \rightarrow 0$, Eqs. (5.3) and (5.4) become horizontal lines, so that any changes in the exogenous variables, leads to a proportional shift along these horizontal lines. In contrast, higher values of 
$\tilde{\varepsilon}_{L}\left(\tilde{\varepsilon}_{H}\right)$ imply a low substitutability between $(H-) L$ - and $M$-technology modes in the neighborhood of $z_{L}\left(z_{H}\right)$. In this case, the curves denoted by Eqs. (5.3) and (5.4) become vertical lines. Consequently, in the limiting case when technology adoption is extremely prohibitive, i.e. $\left\{\tilde{\varepsilon}_{L}, \tilde{\varepsilon}_{H}\right\} \rightarrow \infty$, the relative range of final goods employing technology mode $M$ stays constant.

These results highlight the rich pattern of interaction between immigration, endogenous technology choice, and between consumer preferences and monopolist's market power. They also underline the importance of general equilibrium implications, e.g. the "spillover" effects of low-skill immigration for medium-skilled and high-skilled workers due to the endogenous response of firms to technology choice and changes in the skill mix. Particularly, the results highlight the important role of endogenous technology choice, mitigating the direct labor supply driven wage effects, and thus reconciling the empirical findings discussed in the introduction.

\subsubsection{Medium-skill immigration and distributional effect}

In this section, I discuss the potential wage effects of medium-skilled migration scenario, reflecting recent observations in many old member states of the EU which have experienced a rapid increase in the share of immigrants with medium-skill attainments in the course of EU enlargement towards Eastern and Central European countries (cf. Muysken et al., 2015). To compute the comparative statics, utilize Eqs. (4.34) and (4.35) and differentiate with respect to medium-skilled labor endowment $\left(\mathrm{d} N_{M}>0\right)$ to obtain

$$
\begin{aligned}
\frac{\mathrm{d} \ln \left(w_{L} / w_{M}\right)}{\mathrm{d} \ln N_{M}} & =\frac{\sigma-\epsilon}{(\epsilon-1) \sigma}\left[\Gamma_{M}-1\right], \\
\frac{\mathrm{d} \ln \left(w_{M} / w_{H}\right)}{\mathrm{d} \ln N_{M}} & =-\frac{\sigma-\epsilon}{(\epsilon-1) \sigma}\left[\Delta_{M}-1\right],
\end{aligned}
$$

where $\Gamma_{M} \equiv \frac{\mathrm{d} \ln \left[z_{L} /\left(z_{H}-z_{L}\right)\right]}{\mathrm{d} \ln N_{M}}$ and $\Delta_{M} \equiv \frac{\mathrm{d} \ln \left[\left(z_{H}-z_{L}\right) /\left(1-z_{H}\right)\right]}{\mathrm{d} \ln N_{M}}$ denote the relative change in the range of technology modes due to medium-skill immigration and by Proposition 4.1 $\Gamma_{M}<0$ and $\Delta_{M}>0$. The next proposition summarizes the main results.

Proposition 4.3 (Medium-skill Immigration and Wage Polarization). Immigration of medium-skilled workers generates a market size effect, raising the competition among same skilled workers, and the comparative advantage of the medium-skill complement technology mode and the mass of intermediate goods utilizing medium-skilled workers. If the degree of technology adoption is sufficiently positive and finite, i.e. $0<\left\{\tilde{\varepsilon}_{L}, \tilde{\varepsilon}_{H}\right\}<\infty$, then medium-skilled immigration induce unambiguously a

(i) A polarization effect, where medium-skilled wages decline relative to low-skilled and high-skilled wages, whenever monopolist's market power is higher than the goods demand elasticity, i.e. $\sigma<\epsilon$.

(ii) An increase in medium-skilled wages relative to low-skilled and high-skilled wages, whenever $\sigma>\epsilon$.

Proof. See Appendix 4.A.4. 
The intuition behind the results is similar to the one discussed in Proposition 4.2. However, Proposition 4.3 denotes an important difference to low-skilled and high-skilled immigration scenarios. Medium-skill immigration may potentially generate a polarizing wage effect.

\subsection{Conclusion}

Immigration has a potential impact on the skill structure of the host economy. The existing studies have emphasized the important role of endogenous technology changes, firm heterogeneity, and endogenous choice between different production techniques in explaining the differential trends in wage inequality in many advanced countries over the last decades. One set of studies has highlighted the role of skill-capital complementarity and endogenous technical change, translating changes in the skill mix into changes in factor intensity and to higher returns for those factors. Another set of studies has emphasized the role of endogenous choice of production technology. The implication of this latter channel differs from the former one by indicating that the initial direct supply effect on wages may dissipates over time as immigration induces higher investments by firms in production techniques, complements workers who were affected by immigration.

Yet, we are lacking in understanding the determinants of and the interaction between these two competing arguments. This paper provides a tractable model that allows for a richer structure of the goods market associated with endogenous technology adoption, monopolistic competition and technology-skill complementarity. Moreover, the labor market consists of low-, medium- and high-skilled workers and the production technology exhibits increasing returns to scale. This latter property allows to capture the implications of directed technical change regarding changes skill premium due to immigration-induced changes in the skill mix. In doing so, I investigate jointly the extent and the direction of potential driving forces in the general equilibrium context.

The theoretical analysis uncovers several new insights. First, immigration-induced changes in the domestic wage structure can be decomposed into two key channels: i) a labor supply effect, and ii) a technology adoption effect. Second, the direction of each of these channels depend on the relative magnitude between consumer preference, i.e. goods demand elasticity, and the market power of monopolists, i.e. the elasticity of substitution between intermediates. Third, relative magnitude between these two channels is characterized by the interaction between a direct and an indirect market size effect. On the one hand, immigration-induced changes in the skill structure generates a direct market size effect, associated with higher profitability of producing intermediate goods and hence an increase in the mass of monopolists employing those type of workers. On the other hand, immigration also induces an indirect scale effect, associated with endogenous technology adoption due to immigration-induced changes in the comparative advantages between different technology modes. This latter scale effect effect induces an increase in the relative price of competing goods and hence raises the mass of monopolists in industries sheltered from immigration, indicating a biased price effect for competing technology modes. More interestingly, the analysis reveals that the extent of the indirect scale effect depends crucially on the degree of adoption between the different technology 
modes.

The predictions of the comparative statics regarding impact of immigration on the domestic wage structure reveals the following novel insights. Whenever the consumer preferences are more elastic compared to the market power of monopolists, the market size effect will dominate the biased price effect. Moreover, in the case where the adoption between different technology modes becomes very easy, the domestic wage structure becomes in sensitive to immigration-induced changes in the skill mix. More interestingly, in the empirically plausible scenario, where the market power of monopolists is larger than the goods demand elasticity and the choice between different technology modes is characterized by a sufficient degree of imperfect substitutability, low-skilled (high-skill) immigration induces changes in the wage structure similar to a "skill-biased" ("unskillbiased") technical change, while medium-skilled immigration induces a polarization of the domestic wage structure.

These results highlight the important aspect of general equilibrium implications, capturing a rich set of competing channels. Thus, this framework contributes to the existing literature by highlighting the key features characterizing immigrationinduced changes in the domestic wage structure, which are consistent with empirical observations regarding the patterns of wage inequality in many advanced countries over the last decades. Understanding these adjustment mechanisms has also important policy implications. It allows to response effectively to immigration shock by directing efficiently its impact. 


\section{Appendix}

\section{A Proofs}

\section{A.1 Proof of Lemma 4.1}

First, notice again that perfect competition requires, i.e. $\pi_{k}(z)=0$ for all $z$ and $k=\{L, M, H\}$. Thus, considering two firms using the same technology, we must have

$$
\begin{aligned}
p_{L}(z) \varphi_{L}(z) & =p_{L}\left(z^{\prime}\right) \varphi_{L}\left(z^{\prime}\right), \quad \forall z, z^{\prime} \in\left[0, z_{L}\right] \\
p_{M}(z) \varphi_{M}(z) & =p_{M}\left(z^{\prime}\right) \varphi_{M}\left(z^{\prime}\right), \quad \forall z, z^{\prime} \in\left(z_{L}, z_{H}\right) \\
p_{H}(z) \varphi_{H}(z) & =p_{H}\left(z^{\prime}\right) \varphi_{H}\left(z^{\prime}\right), \quad \forall z, z^{\prime} \in\left[z_{H}, 1\right]
\end{aligned}
$$

This implies that the price of each final good, $p(z)$, adjusts to changes in the productivity of the technology, $\varphi(z)$. Let $P_{k}$ denote the price index of technology mode $k=\{L, M, H\}$, then we can rewrite the price schedule of each technology mode as follows

$$
p_{k}(z)=P_{k} \varphi_{k}(z)^{-1} .
$$

From the optimal demand condition for final goods Eq. (4.3), it can be shown that the total production cost of $Y$ is given by $\int_{0}^{1} p(z) y(z) \mathrm{d}=C_{Y} Y$, where $C_{Y}$ denotes the unit costs, i.e.

$$
C_{Y}=\left[\int_{0}^{z_{L}} p_{L}(z)^{1-\sigma} \mathrm{d}+\int_{z_{L}}^{z_{H}} p_{M}(z)^{1-\sigma} \mathrm{d}+\int_{z_{H}}^{1} p_{H}(z)^{1-\sigma} \mathrm{d}\right]^{\frac{1}{1-\sigma}} .
$$

Now, the optimal choice of cut-off points $z_{L}$ and $z_{H}$ is obtained by minimizing $C_{Y}$ w.r.t. $z_{L}$ and $z_{H}$ :

$$
\begin{aligned}
\frac{\mathrm{d} C_{Y}}{\mathrm{~d} z_{L}}=\frac{1}{1-\sigma} C_{Y}^{\sigma}\left(p_{L}\left(z_{L}\right)^{1-\sigma}-p_{M}\left(z_{L}\right)^{1-\sigma}\right) & =0, \\
\frac{\mathrm{d} C_{Y}}{\mathrm{~d} z_{H}}=\frac{1}{1-\sigma} C_{Y}^{\sigma}\left(p_{M}\left(z_{H}\right)^{1-\sigma}-p_{H}\left(z_{H}\right)^{1-\sigma}\right) & =0 .
\end{aligned}
$$

Using (5.6) for the respective technology mode, we get that $\frac{\mathrm{d} C_{Y}}{\mathrm{~d} z_{L}}=0$ and $\frac{\mathrm{d} C_{Y}}{\mathrm{~d} z_{H}}=0$ if and only if

$$
\begin{aligned}
& \frac{P_{L}}{P_{M}}=\Lambda_{L}\left(z_{L}\right), \quad \Lambda_{L}\left(z_{L}\right) \equiv\left(\frac{\varphi_{L}\left(z_{L}\right)}{\varphi_{M}\left(z_{L}\right)}\right), \\
& \frac{P_{M}}{P_{H}}=\Lambda_{H}\left(z_{H}\right), \quad \Lambda_{H}\left(z_{H}\right) \equiv\left(\frac{\varphi_{M}\left(z_{H}\right)}{\varphi_{H}\left(z_{H}\right)}\right) .
\end{aligned}
$$

To prove that $0<z_{L}<z_{H}<1$, recall from Assumption 4 that

$$
\frac{\mathrm{d} \ln \Lambda_{L}(z)}{\mathrm{d} z}<\frac{\mathrm{d} \ln \Lambda_{H}(z)}{\mathrm{d} z}<0 .
$$

Given this property, there must exist a range of values of the relative price indexes such that $0<z_{L}<$ $z_{H}<1$. It can be easily verified that if $\frac{P_{L}}{P_{M}}>\Lambda_{L}(0)$, then low-tech technology has no comparative advantages to be installed for production of any final good $z \in[0,1]$. Similarly, if $\frac{P_{M}}{P_{H}}<\Lambda_{H}$ (1), it is not cost-efficient to install high-tech technology to produce any final good $z \in[0,1]$. Thus, in Lemma 4.1, Eq. (4.8) provides the range of values of $\frac{P_{L}}{P_{M}}$ and $\frac{P_{M}}{P_{H}}$ that permits the existence of all the three technology modes. Figure 4.5 depicts the equilibrium technology allocation for arbitrary values of relative price indexes that satisfy these conditions. 
Figure 4.5: Equilibrium technology allocation

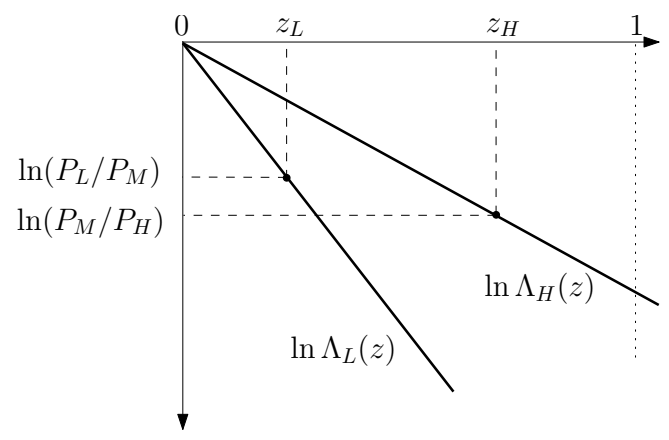

\section{A.2 Proof of Proposition 4.1}

The computation of the comparative statics for the other exogenous changes is similar to the one conducted for changes in the composite factor labor $N_{M}$ due to exogenous variation in marginal offshoring cost and immigration of medium-skilled workers. To do so, recall the implicit $2 \times 2$ system of equations, Eqs. (5.30) and (5.31). Now, differentiating totally this system and applying Cramer's rule, we obtain the following results for exogenous variation in:

- High-skill endowment

$$
\begin{aligned}
\frac{\mathrm{d} \ln z_{L}}{\mathrm{~d} \ln N_{H}} & =\frac{\mathcal{D}_{H}}{\mathcal{D}_{J}}=-\frac{\frac{1}{z_{L}\left(z_{H}-z_{L}\right)}}{\mathcal{D}_{J}}<0, \\
\frac{\mathrm{d} \ln z_{L}}{\mathrm{~d} \ln f_{H}} & =-\frac{\mathrm{d} \ln z_{L}}{\mathrm{~d} \ln N_{H}}>0
\end{aligned}
$$

and

$$
\begin{aligned}
\frac{\mathrm{d} \ln z_{H}}{\mathrm{~d} \ln N_{H}} & =\frac{\mathcal{D}_{H}}{\mathcal{D}_{J}}=-\frac{\frac{\epsilon-1}{\epsilon} \frac{\tilde{\varepsilon}_{L}}{z_{H} z_{L}}+\frac{1}{z_{L}\left(z_{H}-z_{L}\right)}}{\mathcal{D}_{J}}<0 . \\
\frac{\mathrm{d} \ln z_{H}}{\mathrm{~d} \ln f_{H}} & =-\frac{\mathrm{d} \ln z_{H}}{\mathrm{~d} \ln N_{H}}>0
\end{aligned}
$$

- Low-skill endowment

$$
\begin{aligned}
\frac{\mathrm{d} \ln z_{L}}{\mathrm{~d} \ln N_{L}} & =\frac{\mathcal{D}_{L}}{\mathcal{D}_{J}}=\frac{\frac{\epsilon-1}{\epsilon} \frac{\tilde{\varepsilon}_{H}}{z_{L} z_{H}}+\frac{1-z_{L}}{z_{L}\left(1-z_{H}\right)\left(z_{H}-z_{L}\right)}}{\mathcal{D}_{J}}>0, \\
\frac{\mathrm{d} \ln z_{L}}{\mathrm{~d} \ln f_{L}} & =-\frac{\mathrm{d} \ln z_{L}}{\mathrm{~d} \ln N_{L}}<0
\end{aligned}
$$

and

$$
\begin{aligned}
\frac{\mathrm{d} \ln z_{H}}{\mathrm{~d} \ln N_{L}} & =\frac{\mathcal{D}_{L}}{\mathcal{D}_{J}}=\frac{\frac{1}{z_{H}\left(z_{H}-z_{L}\right)}}{\mathcal{D}_{J}}>0 . \\
\frac{\mathrm{d} \ln z_{H}}{\mathrm{~d} \ln f_{L}} & =-\frac{\mathrm{d} \ln z_{H}}{\mathrm{~d} \ln N_{L}}<0
\end{aligned}
$$


where the Jacobi is given by

$$
\begin{aligned}
\mathcal{D}_{J}= & \left(\frac{\epsilon-1}{\epsilon} \frac{\tilde{\varepsilon}_{L}}{z_{L}}+\frac{1}{z_{L}}\right)\left(\frac{\epsilon-1}{\epsilon} \frac{\tilde{\varepsilon}_{H}}{z_{H}}+\frac{1-z_{L}}{\left(1-z_{H}\right)\left(z_{H}-z_{L}\right)}\right) \\
& +\frac{1}{z_{H}-z_{L}}\left(\frac{\epsilon-1}{\epsilon} \frac{\tilde{\varepsilon}_{H}}{z_{H}}+\frac{1}{1-z_{H}}\right)>0
\end{aligned}
$$

Moreover, from (4.A.39) and (4.A.41) it can be readily verified that

$$
\frac{\mathrm{d} \ln z_{H}-\mathrm{d} \ln z_{L}}{\mathrm{~d} \ln N_{H}}=-\frac{1}{\mathcal{D}_{J}}\left(\frac{\epsilon-1}{\epsilon} \frac{\tilde{\varepsilon}_{L}}{z_{H} z_{L}}\right)<0
$$

and similarly from (4.A.43) and (4.A.45)

$$
\frac{\mathrm{d} \ln z_{H}-\mathrm{d} \ln z_{L}}{\mathrm{~d} \ln N_{L}}=-\frac{1}{\mathcal{D}_{J}}\left(\frac{\epsilon-1}{\epsilon} \frac{\tilde{\varepsilon}_{H}}{z_{H} z_{L}}+\frac{1}{z_{H} z_{L}\left(1-z_{H}\right)}\right)<0
$$

\section{A.3 Proof of Proposition 4.2}

\section{Low-skilled immigration}

To obtain explicit solution for the terms $\Gamma_{L} \equiv \frac{\mathrm{d} \ln \left[z_{L} /\left(z_{H}-z_{L}\right)\right]}{\mathrm{d} \ln N_{L}}$ and $\Delta_{L} \equiv \frac{\mathrm{d} \ln \left[\left(z_{H}-z_{L}\right) /\left(1-z_{H}\right)\right]}{\mathrm{d} \ln N_{L}}$, we can utilize the results of the comparative statics in Eqs. (4.A.43) and (4.A.45) to obtain the following terms

$$
\begin{aligned}
\Gamma_{L} & =\frac{z_{H}}{z_{H}-z_{L}}\left(\frac{\mathrm{d} \ln z_{L}-\mathrm{d} \ln z_{H}}{\mathrm{~d} \ln N_{L}}\right) \\
& =\frac{1}{\mathcal{D}_{J}}\left(\frac{\epsilon-1}{\epsilon} \frac{\tilde{\varepsilon}_{H}}{z_{L}\left(z_{H}-z_{L}\right)}+\frac{1}{z_{L}\left(1-z_{H}\right)\left(z_{H}-z_{L}\right)}\right)>0
\end{aligned}
$$

and

$$
\begin{aligned}
\Delta_{L} & =-\left(\frac{z_{L}}{z_{H}-z_{L}} \frac{\mathrm{d} \ln z_{L}}{\mathrm{~d} \ln N_{L}}-\frac{z_{H}\left(1-z_{L}\right)}{\left(1-z_{H}\right)\left(z_{H}-z_{L}\right)} \frac{\mathrm{d} \ln z_{H}}{\mathrm{~d} \ln N_{L}}\right) \\
& =-\frac{1}{\mathcal{D}_{J}}\left(\frac{z_{L}}{z_{H}-z_{L}}\left(\frac{\epsilon-1}{\epsilon} \frac{1-z_{L}}{z_{L} z_{H}}+\frac{z_{H}\left(1-z_{L}\right)}{z_{L}\left(1-z_{H}\right)\left(z_{H}-z_{L}\right)}\right)-\frac{1}{\left(1-z_{H}\right)\left(z_{H}-z_{L}\right)} \frac{1}{z_{H}\left(z_{H}-z_{L}\right)}\right) \\
& =-\frac{1}{\mathcal{D}_{J}}\left(\frac{\epsilon-1}{\epsilon} \frac{\tilde{\varepsilon}_{H}}{\left(z_{H}-z_{L}\right) z_{H}}\right)<0
\end{aligned}
$$

It is immediately evident from Eqs. (4.A.50) and (4.A.51) that the extent of changes in the technology margins $z_{L}$ and $z_{H}$ depends crucially on the elasticity of technology adoption in the neighborhood of $z_{L}$ $\left(\tilde{\varepsilon}_{L}\right)$ and $z_{H}\left(\tilde{\varepsilon}_{H}\right)$. We can distinguish between several cases.

If it is extremely difficult for final good producer to replace technology mode $M$ by $L$ in the neighborhood of $z_{L}$, then it follows

$$
\left.\lim _{\tilde{\varepsilon}_{L} \rightarrow \infty} \mathcal{D}_{J}\right|_{\tilde{\varepsilon}_{H}>0}=\left.\infty \Rightarrow \lim _{\tilde{\varepsilon}_{L} \rightarrow \infty} \Gamma_{L}\right|_{\tilde{\varepsilon}_{H}>0}=\left.\Delta_{L}\right|_{\tilde{\varepsilon}_{H}>0}=0,
$$

implying no changes at the extensive margin. In other extreme case, where technology adoption is very 
easy, we obtain

$$
\begin{aligned}
\lim _{\left\{\tilde{\varepsilon}_{H}, \tilde{\varepsilon}_{L}\right\} \rightarrow 0} \mathcal{D}_{J} & =\frac{1}{z_{L}\left(z_{H}-z_{L}\right)\left(1-z_{H}\right)} \\
\Rightarrow \lim _{\left\{\tilde{\varepsilon}_{H}, \tilde{\varepsilon}_{L}\right\} \rightarrow 0} \Gamma_{L} & =1 \\
\Rightarrow \lim _{\left\{\tilde{\varepsilon}_{H}, \tilde{\varepsilon}_{L}\right\} \rightarrow 0} \Delta_{L} & =0 .
\end{aligned}
$$

This implies that changes in the technology margin $z_{L}$ is proportional to changes in the low-skilled labor supply and so does the relative range between low-skill and medium-skill technology modes, while the usage of medium-skill technology mode relative to high-skill technology mode stays constant.

Hence, for an intermediate range of values of the parameters, $0<\left\{\tilde{\varepsilon}_{L}, \tilde{\varepsilon}_{H}\right\}<\infty$, i.e. for a sufficient degree of imperfect substitutability between technology modes,

$$
0<\Gamma_{L}<1, \quad 0<\left|\Delta_{L}\right|<1
$$

\section{High-skilled immigration}

To obtain explicit solution for the terms $\Gamma_{H} \equiv \frac{\mathrm{d} \ln \left[z_{L} /\left(z_{H}-z_{L}\right)\right]}{\mathrm{d} \ln N_{H}}$ and $\Delta_{H} \equiv \frac{\mathrm{d} \ln \left[\left(z_{H}-z_{L}\right) /\left(1-z_{H}\right)\right]}{\mathrm{d} \ln N_{H}}$, we can utilize the results of the comparative statics in Eqs. (4.A.39) and (4.A.41) to obtain the following terms

$$
\begin{aligned}
\Gamma_{H} & =\frac{z_{H}}{z_{H}-z_{L}}\left(\frac{\mathrm{d} \ln z_{L}-\mathrm{d} \ln z_{H}}{\mathrm{~d} \ln N_{H}}\right) \\
& =\frac{1}{\mathcal{D}_{J}}\left(\frac{\epsilon-1}{\epsilon} \frac{\tilde{\varepsilon}_{L}}{\left(z_{H}-z_{L}\right) z_{L}}\right)>0
\end{aligned}
$$

and

$$
\begin{aligned}
\Delta_{H} & =\left(\frac{z_{H}\left(1-z_{L}\right)}{\left(1-z_{H}\right)\left(z_{H}-z_{L}\right)} \frac{\mathrm{d} \ln z_{H}}{\mathrm{~d} \ln N_{H}}-\frac{z_{L}}{z_{H}-z_{L}} \frac{\mathrm{d} \ln z_{L}}{\mathrm{~d} \ln N_{H}}\right) \\
& =-\frac{1}{\mathcal{D}_{J}}\left(\frac{z_{H}\left(1-z_{L}\right)}{\left(1-z_{H}\right)\left(z_{H}-z_{L}\right)}\left(\frac{\epsilon-1}{\epsilon} \frac{\tilde{\varepsilon}_{L}}{z_{H} z_{L}}+\frac{1}{z_{L}\left(z_{H}-z_{L}\right)}\right)-\frac{z_{L}}{z_{H}-z_{L}} \frac{1}{z_{L}\left(z_{H}-z_{L}\right)}\right) \\
& =-\frac{1}{\mathcal{D}_{J}}\left(\frac{\epsilon-1}{\epsilon} \frac{\tilde{\varepsilon}_{L}\left(1-z_{L}\right)}{\left(1-z_{H}\right)\left(z_{H}-z_{L}\right) z_{L}}+\frac{1}{z_{L}\left(z_{H}-z_{L}\right)\left(1-z_{H}\right)}\right)<0
\end{aligned}
$$

High-skill immigration induces an opposite effect compared to low-skill immigration. Now, it is immediately evident from Eqs. (4.A.52) and (4.A.53) that the magnitude of changes in the technology margins $z_{L}$ and $z_{H}$ now depends crucially on the elasticity of technology adoption at the margin $z_{H}, \tilde{\varepsilon}_{H}$. Thus, if it is extremely difficult for final good producer to replace technology mode $M$ by $H$, it follows

$$
\left.\lim _{\tilde{\varepsilon}_{H} \rightarrow \infty} \mathcal{D}_{J}\right|_{\tilde{\varepsilon}_{L}>0}=\left.\infty \Rightarrow \lim _{\tilde{\varepsilon}_{H} \rightarrow \infty} \Gamma_{H}\right|_{\tilde{\varepsilon}_{L}>0}=\left.\Delta_{H}\right|_{\tilde{\varepsilon}_{L}>0}=0 .
$$

In other extreme case, where technology adoption is very easy, we obtain

$$
\begin{aligned}
\lim _{\left\{\tilde{\varepsilon}_{H}, \tilde{\varepsilon}_{L}\right\} \rightarrow 0} \mathcal{D}_{J} & =\frac{1}{z_{L}\left(z_{H}-z_{L}\right)\left(1-z_{H}\right)} \\
\Rightarrow \lim _{\left\{\tilde{\varepsilon}_{H}, \tilde{\varepsilon}_{L}\right\} \rightarrow 0} \Gamma_{H} & =0 \\
\Rightarrow \lim _{\left\{\tilde{\varepsilon}_{H}, \tilde{\varepsilon}_{L}\right\} \rightarrow 0} \Delta_{H} & =1 .
\end{aligned}
$$

This implies that changes in the technology margin $z_{H}$ is proportional to changes in the high-skilled labor supply and so does the relative range between high-skill and medium-skill technology modes, while the usage of medium-skill technology mode relative to low-skill technology mode stays constant.

Hence, for an intermediate range of values of the parameters, $\infty>\left\{\tilde{\varepsilon}_{L}, \tilde{\varepsilon}_{H}\right\}>0$, i.e. for a sufficient 
degree of imperfect substitutability between technology modes,

$$
0<\Gamma_{H}<1, \quad 0<\Delta_{H}<1 .
$$

\section{A.4 Proof of Proposition 4.3}

To derive the explicit solution for $\Gamma_{M} \equiv \frac{\mathrm{d} \ln \left[z_{L} /\left(z_{H}-z_{L}\right)\right]}{\mathrm{d} \ln N_{M}}$ and $\Delta_{M} \equiv \frac{\mathrm{d} \ln \left[\left(z_{H}-z_{L}\right) /\left(1-z_{H}\right)\right]}{\mathrm{d} \ln N_{M}}$, utilize the results of the comparative statics in Eqs. (4.30) and (4.31) to obtain the following terms

$$
\begin{aligned}
\Gamma_{M} & =-\frac{z_{H}}{z_{H}-z_{L}}\left(\frac{\mathrm{d} \ln z_{H}-\mathrm{d} \ln z_{L}}{\mathrm{~d} \ln N_{M}}\right) \\
& =-\frac{1}{\mathcal{D}_{J}}\left(\frac{1}{z_{L}\left(z_{H}-z_{L}\right)\left(1-z_{H}\right)}+\frac{\epsilon-1}{\epsilon} \frac{\tilde{\varepsilon}_{L}+\tilde{\varepsilon}_{H}}{\left(z_{H}-z_{L}\right) z_{L}}\right)<0
\end{aligned}
$$

and

$$
\begin{aligned}
\Delta_{M} & =\frac{z_{H}\left(1-z_{L}\right)}{\left(1-z_{H}\right)\left(z_{H}-z_{L}\right)} \frac{\mathrm{d} \ln z_{H}}{\mathrm{~d} \ln N_{M}}-\frac{z_{L}}{z_{H}-z_{L}} \frac{\mathrm{d} \ln z_{L}}{\mathrm{~d} \ln N_{M}} \\
& =\frac{1}{\mathcal{D}_{J}}\left(\frac{z_{H}\left(1-z_{L}\right)}{\left(1-z_{H}\right)\left(z_{H}-z_{L}\right)}\left(\frac{1}{z_{H} z_{L}}+\frac{\epsilon-1}{\epsilon} \frac{\tilde{\varepsilon}_{L}}{z_{H} z_{L}}\right)+\frac{z_{L}}{z_{H}-z_{L}}\left(\frac{1}{\left(1-z_{H}\right) z_{L}}+\frac{(\epsilon-1) \tilde{\varepsilon}_{H}}{\epsilon z_{H} z_{L}}\right)\right) \\
& =\frac{1}{\mathcal{D}_{J}}\left(\frac{1}{\left(1-z_{H}\right)\left(z_{H}-z_{L}\right) z_{L}}+\frac{\epsilon-1}{\epsilon}\left(\frac{\left(1-z_{L}\right)}{\left(1-z_{H}\right)\left(z_{H}-z_{L}\right)} \frac{\tilde{\varepsilon}_{L}}{z_{L}}+\frac{1}{z_{H}-z_{L}} \frac{\tilde{\varepsilon}_{H}}{z_{H}}\right)\right)>0
\end{aligned}
$$

where the Jacobi is given by Eq. (5.A.89). Now, taking the limits of the elasticities $\tilde{\varepsilon}_{L}$ and $\tilde{\varepsilon}_{H}$, it can be readily verified

$$
\begin{aligned}
& \lim _{\left\{\tilde{\varepsilon}_{L}, \tilde{\varepsilon}_{H}\right\} \rightarrow 0} \mathcal{D}_{J}=\frac{1}{\left(1-z_{H}\right)\left(z_{H}-z_{L}\right) z_{L}} \Rightarrow \lim _{\left\{\tilde{\varepsilon}_{L}, \tilde{\varepsilon}_{H}\right\} \rightarrow 0} \Gamma_{M}=-\Delta_{M}=-1 \\
& \lim _{\left\{\tilde{\varepsilon}_{L}, \tilde{\varepsilon}_{H}\right\} \rightarrow \infty} \mathcal{D}_{J}=\infty \quad \Rightarrow \lim _{\left\{\tilde{\varepsilon}_{L}, \tilde{\varepsilon}_{H}\right\} \rightarrow \infty} \Gamma_{M}=\Delta_{M}=0
\end{aligned}
$$

\section{B Derivation of equilibrium solutions}

\section{B.1 Demand for intermediate goods}

Any firm in the final goods market chooses the optimal amount of intermediate goods by minimizing the production cost

$$
C_{k}=\int_{0}^{A_{k}} p_{k}(j) x_{k}(j) \mathrm{d} j, \forall k=\{L, M, H\}
$$

subject to CES aggregate (5.7). The optimization programming can be defined by the following Lagrangian:

$$
\min _{x_{k}(j)} \mathcal{L}_{k}=\int_{0}^{A_{k}} p_{k}(j) x_{k}(j) \mathrm{d} j-\zeta_{k}\left(X_{k}-\left[\int_{0}^{A_{k}} x_{k}(j)^{\frac{\epsilon-1}{\epsilon}}\right]^{\frac{\epsilon}{\epsilon-1}}\right),
$$

where $\zeta_{k}$ denotes the Lagrangian multiplier. From the first-order condition

$$
\frac{\partial \mathcal{L}_{k}}{\partial x_{k}(j)}=p_{k}(j)-\zeta_{k} X_{k}^{1 / \epsilon} x_{k}(j)^{-1 / \epsilon}=0,
$$


we obtain the optimal inverse demand condition for each intermediate goods produced under technology mode $k$. Utilizing (4.B.56) into the constraint, Eq. (5.7), the Lagrangian multiplier denotes the "cost index" of the composite good

$$
\zeta_{k}=\left[\int_{0}^{A_{k}} p_{k}(j)^{1-\epsilon} \mathrm{d} j\right]^{\frac{1}{1-\epsilon}},
$$

and inserting (4.B.56) back into the cost function yields

$$
C_{k}=\zeta_{k} X_{k}
$$

\section{B.2 Derivation of the mass of monopolists}

Notice that the perfectly competitive nature of the final goods market requires zero profits, i.e.

$$
p_{k}(z) y_{k}(z)=\zeta_{k} X_{k}, \quad \forall k=\{L, M, H\}
$$

Moreover, using Eqs. (4.2) and (4.5), it follows that in any competitive equilibrium the price index of technology mode $k$ must equal its marginal cost, i.e.

$$
P_{k}=\zeta_{k}
$$

Now, utilizing the equilibrium value of $\zeta_{k}$ from Eq. (4.17), yields

$$
P_{k}=\zeta_{k}=\frac{\epsilon}{\epsilon-1} A_{k}^{\frac{1}{1-\epsilon}} w_{k} .
$$

Then, the total budget constraint of any final good producer $z \in \mathcal{S} k$ requires

$$
p(z) y(z)=w_{k} n_{k}=\frac{w_{k} N_{k}}{\mathcal{S}_{k}},
$$

where the second equality follows from Eqs. (5.17)-(5.19), for the respective technology mode.

Utilize Eqs. (4.2) and (4.5) in the left hand side of the previous equation and manipulate to obtain

$$
P_{k} A_{k}^{\frac{\epsilon}{\epsilon-1}} x_{k}=\frac{w_{k} N_{k}}{\mathcal{S}_{k}} .
$$

Next, use the FE condition (5.14), and Eq. (5.B.101) to substitute for $x_{k}$ and $P_{k}$ in the previous equation, respectively, so that after some manipulation we obtain

$$
A_{k} \epsilon f_{k} w_{k}=\frac{w_{k} N_{k}}{\mathcal{S}_{k}}
$$

Rearranging and solving w.r.t. $A_{k}$ yields the equilibrium mass of monopolists in the respective technology mode.

\section{B.3 Derivation of aggregate sectoral output}

The derivation of the aggregate sectoral output is illustrated for technology mode complementing lowskilled workers. The aggregation over the range of all final goods using the other two types of technology can be derived similarly. Define the total cost using low-skilled technology as

$$
P_{L} Y_{L}=\int_{0}^{z_{L}} p(z) y(z) \mathrm{d} z
$$


Utilizing Eqs. (4.5) and (4.23) yields

$$
\begin{aligned}
P_{L} Y_{L} & =\int_{0}^{z_{L}} p(z) \varphi_{L}(z) A_{L}^{\frac{1}{\epsilon-1}} \frac{N_{L}}{z_{L}} \mathrm{~d} z, \\
& =\int_{0}^{z_{L}} P_{L} A_{L}^{\frac{\epsilon}{\epsilon-1}} \frac{N_{L}}{z_{L}} \mathrm{~d} z .
\end{aligned}
$$

Manipulating slightly, we get

$$
Y_{L}=A_{L}^{\frac{1}{\epsilon-1}} N_{L}
$$

Following the same steps, we obtain

$$
\begin{aligned}
Y_{M} & =A_{M}^{\frac{1}{\epsilon-1}} N_{M}, \\
Y_{H} & =A_{H}^{\frac{1}{\epsilon-1}} N_{H}
\end{aligned}
$$

\section{B.4 Derivation of relative wages}

The relative wage between low- and medium-skilled workers $\left(w_{L} / w_{M}\right)$ can be derived as follows. First, recall the optimal final-goods demand condition (4.3) and take rations with respect to $M$ to obtain

$$
\begin{aligned}
y_{L}(z) / y_{M}\left(z^{\prime}\right) & =\left(p_{L}(z) / p_{M}\left(z^{\prime}\right)\right)^{-\sigma} \\
y_{M}\left(z^{\prime}\right) / y_{H}\left(z^{\prime \prime}\right) & =\left(p_{M}\left(z^{\prime}\right) / p_{H}\left(z^{\prime \prime}\right)\right)^{-\sigma}
\end{aligned}
$$

for any $z \in\left[0, z_{L}\right), z^{\prime} \in\left(z_{L}, z_{H}\right)$, and $z^{\prime \prime} \in\left(z_{H}, 1\right]$. It is important to notice that at the cut-off margins $z_{L}$ and $z_{H}$ the relative demand for final goods is undefined since at those margins the planer is indifferent between the different technology modes. Now substituting Eqs. (4.5) and (4.23) into the previously derived equations yields

$$
\begin{aligned}
& \frac{P_{L}}{P_{M}}=\left(\frac{z_{L}}{z_{H}-z_{L}}\right)^{\frac{1}{\sigma}}\left(\frac{Y_{L}}{Y_{M}}\right)^{-\frac{1}{\sigma}} \Lambda_{L}\left(z, z^{\prime}\right)^{\frac{\sigma-1}{\sigma}}=\left(\left[\frac{A_{L}}{A_{M}}\right]^{\frac{1}{\epsilon-1}} \frac{N_{L}}{N_{M}}\left[\frac{z_{L}}{z_{H}-z_{L}}\right]^{-1}\right)^{-\frac{1}{\sigma}} \Lambda_{L}\left(z, z^{\prime}\right)\left(\frac{\tilde{q}}{4 . \mathrm{B}}, 61\right) \\
& \frac{P_{M}}{P_{H}}=\left(\frac{z_{H}-z_{L}}{1-z_{H}}\right)^{\frac{1}{\sigma}}\left(\frac{Y_{M}}{Y_{H}}\right)^{-\frac{1}{\sigma}} \Lambda_{H}\left(z^{\prime}, z^{\prime \prime}\right)^{\frac{\sigma-1}{\sigma}}=\left(\left[\frac{A_{M}}{A_{H}}\right]^{\frac{1}{\epsilon-1}} \frac{N_{M}}{N_{H}}\left[\frac{z_{H}-z_{L}}{1-z_{H}}\right]^{-1}\right)^{-\frac{1}{\sigma}} \Lambda_{H}\left(z^{\prime},\left(k^{\prime} .\right)^{\circ} \cdot \frac{1}{6} 2\right)
\end{aligned}
$$

where $\Lambda_{L}\left(z, z^{\prime}\right) \equiv \frac{\varphi_{L}(z)}{\varphi_{M}\left(z^{\prime}\right)}$ and $\Lambda_{H}\left(z^{\prime}, z^{\prime \prime}\right) \equiv \frac{\varphi_{M}\left(z^{\prime}\right)}{\varphi_{H}\left(z^{\prime \prime}\right)}$ are constants.

Next, recall the zero profit condition (4.B.59) for the final good producers and take the ratio between technology mode $k=L$ and $k=M$ and between technology mode $k=M$ and $k=H$ to obtain, respectively,

$$
\begin{aligned}
\frac{\zeta_{L}}{\zeta_{M}} & =\frac{P_{L}}{P_{M}}, \\
\frac{\zeta_{M}}{\zeta_{H}} & =\frac{P_{M}}{P_{H}} .
\end{aligned}
$$

Substituting Eq. (5.B.105) for $P_{L} / P_{M}$ into Eq. (4.B.63) and inserting the outcome into Eq. (4.32) yields

$$
\frac{w_{L}}{w_{M}}=\left(\left[\frac{A_{L}}{A_{M}}\right]^{\frac{1}{\epsilon-1}} \frac{N_{L}}{N_{M}}\left[\frac{z_{L}}{z_{H}-z_{L}}\right]^{-1}\right)^{-\frac{1}{\sigma}}\left(\frac{X_{L}}{X_{M}}\right)^{1 / \epsilon}\left(\frac{x_{L}}{x_{M}}\right)^{-1 / \epsilon} .
$$

Notice that due to symmetry assumption it follows from (5.7) $X_{k}=A_{k}^{\frac{\epsilon}{\epsilon-1}} x_{k}$. Recall from Eqs. (4.18) and (4.19) the equilibrium values of $A_{L}$ and $A_{M}$ and utilize them in the previous equation, respectively, 
and manipulating slightly to obtain

$$
\frac{w_{L}}{w_{M}}=\left(\frac{N_{L}}{N_{M}}\right)^{\frac{\sigma-\epsilon}{(\epsilon-1) \sigma}}\left(\frac{z_{L}}{z_{H}-z_{L}}\right)^{-\frac{\sigma-\epsilon}{(\epsilon-1) \sigma}} \mathcal{K}_{L, M} .
$$

where $\mathcal{K}_{L, M} \equiv\left(\frac{f_{L}}{f_{M}}\right)^{-\frac{\sigma-1}{(\epsilon-1) \sigma}} \Lambda_{L}\left(z, z^{\prime}\right)^{\frac{\sigma-1}{\sigma}}$. Thus, the relative wage rates between low-skill and mediumskill workers can be decomposed into the following terms

$$
\frac{w_{L}}{w_{M}}=\left(\frac{z_{L}}{z_{H}-z_{L}}\right)^{-\frac{\sigma-\epsilon}{(\epsilon-1) \sigma}} N_{L}^{\frac{\sigma-\epsilon}{(\epsilon-1) \sigma}}\left(N_{M}\right)^{-\frac{\sigma-\epsilon}{(\epsilon-1) \sigma}} \mathcal{K}_{L, M} .
$$

Following similar steps, i.e. (5.B.106) for $P_{M} / P_{H}$ into Eq. (4.B.64) and inserting the outcome into Eq. (4.33) yields the relative wage rate between medium-skill and high-skill workers derived in the text:

$$
\frac{w_{M}}{w_{H}}=\left(\frac{z_{H}-z_{L}}{1-z_{H}}\right)^{-\frac{\sigma-\epsilon}{(\epsilon-1) \sigma}} N_{H}^{-\frac{\sigma-\epsilon}{(\epsilon-1) \sigma}}\left(N_{M}\right)^{\frac{\sigma-\epsilon}{(\epsilon-1) \sigma}} \mathcal{K}_{H, M}
$$

where $\mathcal{K}_{H, M} \equiv\left(\frac{f_{M}}{f_{H}}\right)^{-\frac{\sigma-1}{(\epsilon-1) \sigma}} \Lambda_{H}\left(z^{\prime}, z^{\prime \prime}\right)^{\frac{\sigma-1}{\sigma}}$. 



\section{Immigration, Offshoring, Tasks and Technology Adoption: Implications for Native Wage Structure ${ }^{1}$}

\section{$5.1 \quad$ Introduction}

Immigration and the relocation of jobs abroad by multinationals, i.e. offshoring of domestic jobs, are often blamed for adverse labor market outcomes for native workers. Yet, the empirical findings on the impact of these two aspects of globalization of labor services on wages and employment of native workers are ambiguous. The literature provides several explanations for this. On the one hand, there is a labor supply effect, indicating the direct displacement effect due to increasing labor market competition. On the other hand, there are several indirect channels which may offset the direct adverse effect.

Recent studies have emphasized one salient feature associated with a cost-efficiency enhancing effect of the production process due to reallocation of jobs (tasks) from natives to immigrants (characterized by high intensity in manual, non-interactive content of tasks, cf. Peri (2012); Peri and Sparber (2009)) and to offshore workers (characterized by high intensity in routine, easily codifiable content of tasks, cf. Blinder (2009a); Grossman and Rossi-Hansberg (2008)). The rationale behind this efficiency effect is in the spirit of Ricardian comparative advantage and Smithian specialization forces. ${ }^{2}$ Another potential indirect mechanism proposed in the literature is through directed technological changes (cf. Acemoglu, 2002a) or the adoption of production techniques (cf. Beaudry et al., 2010). The idea is that immigration-induced increase in some production factors lead firms to

\footnotetext{
${ }^{1}$ I am grateful for valuable comments and discussions to Joan Muysken and Thomas Ziesemer. I would also like to thank participants at ETSG 2015 Paris.

${ }^{2}$ Since factor labor is sector/firm specific, cost-efficiency gains induced by immigration and offshoring are associated with factor-augmenting productivity effects. Thus, in what follows the terms productivity effect and efficiency effect are used interchangeably.
} 
adopt production technologies that are more efficient and intensive in the use of that factor (cf. Dustmann and Glitz, 2012; Gauthier-Loiselle and Hunt, 2008; González and Ortega, 2011; Lewis, 2003). ${ }^{3}$ Thus, these endogenous adjustments (task-skill reallocation and choice of production techniques) highlight the efficiency gains induced by either type of globalization of labor that may paradoxically benefit all workers. This evidence has elicited the need for an alternative theoretical paradigm that allows for a richer microfounded model regarding differential specialization patterns between immigrants and natives, or between jobs produced at home and abroad. This paper provides an integrated theoretical framework that allows to jointly investigate the distributional impact of immigration and offshoring, and to analyze the adjustment mechanisms and their underlying determinants.

Formally, I develop the key features of the general equilibrium model in the following steps. First, the model economy is considered that features a rich structure of goods and labor markets, characterized by firm heterogeneity, endogenous technology adoption, monopolistic competition, skill heterogeneity, and technology-skill complementarity. Moreover, due to endogenous changes in the mass of monopolists, the specialization pattern generates increasing returns to scale similar to (a multi-sector version of) semi-end- ogenous growth models as in Romer (1987). ${ }^{4}$ Second, the internal work organization is captured by endogenous task-allocation mechanisms between immigrants and natives, where consistent with empirical observations tasks are ordered according to their communication, interpersonal intensity, so that immigrants specialize in low index tasks, while natives perform the higher indexed tasks. In fact, Ottaviano et al. (2013) show for the U.S. that immigrants are proportionately more in jobs with high content of manual tasks, while natives are concentrated in jobs with a high intensity of communication and complexity content of tasks, see Figure 5.1. In this case, immigrationinduced labor supply shock exhibits a dynamic characteristic, captured by an endogenous response of natives in more communication intensive tasks. Finally, the framework is extended to allow for offshoring in terms of fragmentation of domestic jobs (intermediates) abroad in the spirit of models of international fragmentation of the production process along the value added chain, or trade in intermediate inputs (cf. Feenstra and Hanson, 1999; Jones and Kierzkowski, 2001; Kohler, 2004a; Rodríguez-Clare, 2010). In this way, we are able to capture various aspects of recent trends in globalization of labor by integrating important features of different strands in the literature.

Task allocation and offshoring induce several noteworthy consequences in this environment. First, immigration and offshoring induce two counteracting scale externalities. One the one hand, they induce a direct market-size effect by raising the skill endowment in the intermediate goods sector and the number of intermediate variety, respectively. This is in turn associated with endogenous changes in the mass of intermediate firms employing those factors most intensively - capturing the notion of directed technological change as in Acemoglu (2002b). On the other hand, there is endogenous technology adoption of complementing production modes - reflecting a

\footnotetext{
${ }^{3}$ In a recent study Ghosh et al. (2014) examine the impact of changes in the H-1B cap on the performance of US firms, and find that relaxing the cap on the $\mathrm{H}-1 \mathrm{~B}$ visas raises the average labor productivity, the size and profits of firms contacting heavily R\&D.

${ }^{4}$ See chapter 4 for elaborate formal discussions regarding the wage effects of immigration and the implications of endogenous technology adoption under increasing returns to scale.
} 
Figure 5.1: Immigrants and task specialization

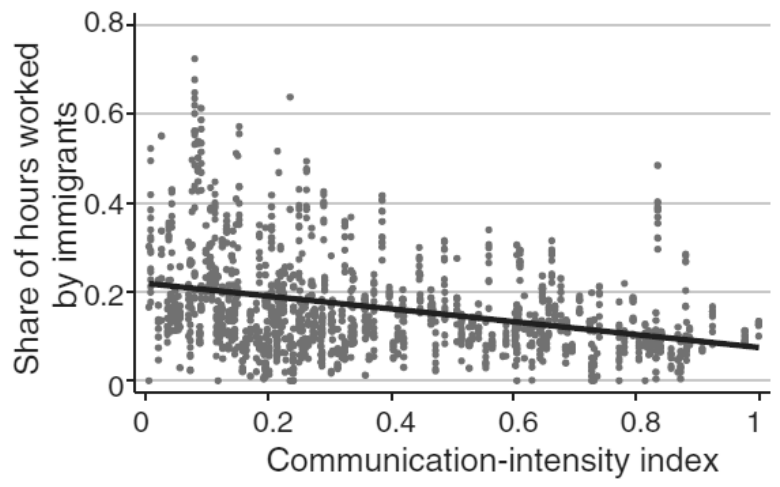

(a) Immigrants and communication intensity

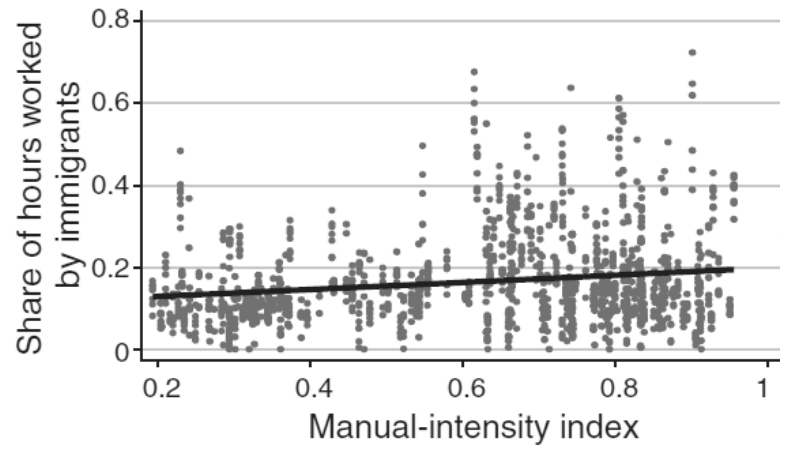

(b) Immigrants and manual intensity

Notes: Communication intensity measures the ability regarding written and oral expression and understanding; manual intensity measures the ability regarding dexterity, strength, and coordination. The slope of the regression line (standard error) in Panel (a): -0.14 (0.01), in Panel (b): 0.087 (0.01). Source: Ottaviano et al. (2013).

technology adoption effect. Thisindirect effect, emerging from the expansion of the range of final goods, tends to counteract the direct effect by increasing the relative price of goods produced under the competing technology modes - an effect analog to the notion of internal and external externalities as in Ethier (1982).

The key feature of this approach is its convenient structure allowing to obtain strong analytical results and precise conditions to sign the three channels (i) the technology adoption effect, (ii) the market size effect, and (iii) the efficiency effect as well as the interaction between them, denoting another novelty of this paper. First, the direction of each of these channels is determined by the relative strength between goods demand elasticity and the market power of monopolists. Second, the relative magnitude between the technology adoption and market size effects depends on the degree of substitutability between different technology modes. Finally, the relative strength of efficiency effect induced by immigration (through task-reallocation) and offshoring (through job reallocation) depends crucially on the initial share of tasks allocated to immigrants and the initial of offshoring rate. At initial low levels, the productivity effect becomes unambiguously the dominating force. Thus, offshoring of medium skill-intensive 
jobs (intermediates) generate similar wage distributional effects to task reallocation induced by medium-skilled immigration.This analogy, to the best of my knowledge, is novel.

Furthermore, the comparative statics analysis uncovers several new insights regarding the potential impact of globalization of labor on native wage structure. In the empirically more relevant case where the market power of monopolists (i.e. the elasticity of substitution between intermediates) is larger than the goods demand elasticity, lowskill immigration induces a wage effect similar to unskill-biased technological change, associated with a monotonic decline in the skill premium across the skill groups, while high-skill immigration induces a wage effect similar to skill-augmenting to technological changes. The wage distributional impact of medium-skill immigration and offshoring has a non-monotonic feature. More precisely, they induce a hump-shaped wage effect implied by high efficiency gains whenever the extent of exposure is initially low, while at initial high levels of exposure changes natives relative wage structure are characterized by a U-shaped relationship.

Although this paper does not account for other important factors like endogenous skill supply, labor market frictions, or institutional factors, the results discussed above highlight the rich pattern of interacting adjustment mechanisms and are consistent with the broad empirical observations of various points in time registering changes in the wage structure in many advanced over the last decades. Thus, this framework highlights the departure from recent immigration and offshoring literature (cf. Acemoglu et al., 2012; Olney, 2012; Ottaviano et al., 2013), which are discussed below.

The rest of the paper is organized as follows. In the next section, the related literature is reviewed. The basic setup of the theoretical framework together with the elaborating discussion of task-based approach are presented in section 5.3. The characteristics of the equilibrium are illustrated in section 5.4, followed by the comparative static analysis of the impact of immigration on native wage structure in section 5.5. The extension of the model with respect to offshoring medium skill-intensive jobs and the analysis of its distributional effect for native workers are elaborated in section 5.6. Finally, concluding remarks are provided in section 5.7.

\subsection{Related literature}

There exists a large body of studies that have sought to analyze the labor market impacts of immigration and offshoring. This paper contributes to different strands in the literature. First, it is a contribution to a growing but still small literature addressing jointly wage an employment effects of immigration and offshoring (cf. Olney, 2012; Ottaviano et al., 2013; Rodríguez-Clare, 2010). The main point of departure to these studies is the endogenous adoption behavior between different production techniques. For example, guided by the theoretical task-based approach à la Grossman and RossiHansberg (2008), Olney (2012) and Ottaviano et al. (2013) analyze empirically the direct and indirect effects of offshoring and immigration for the United States. Imposing implicitly perfect substitutability between immigrants and natives, Olney (2012) finds opposite effects where immigration hurts low-skilled native workers through higher labor 
market competition, while offshoring induces a positive demand effect for the domestic low-skilled labor, driven by the indirect efficiency improvements in the production process. Contrary, using the general task-assignment model of Acemoglu and Autor (2011), Ottaviano et al. (2013) allow immigrants and natives to specialize in different tasks, and find that immigration and offshoring both generate efficiency effects for native workers. ${ }^{5}$

Second, this paper is also contributing to the large literature on the theoretical determinants of offshoring- and technology-induced changes in the wage structure (cf. Acemoglu and Autor, 2011; Acemoglu et al., 2012; Acemoglu and Zilibotti, 2001; Egger et al., 2013; Grossman and Rossi-Hansberg, 2008). ${ }^{6}$ Closely related to this paper is the study by Acemoglu et al. (2012), who investigate the impact of offshoring on directed technological changes and skill premium. The results in this paper share similar features to their analytical findings. For instance, they show that offshoring induces an efficiency effect, and its magnitude depends on the level of the offshoring rate. The offshoringinduced changes in the wage structure are the results of endogenous technological changes, where the direction of this effect is crucially affected by the interaction between goods demand elasticity and the elasticity of substitution between intermediates. However, the structure and the underlying determinants of the mechanism analyzed in this paper differ in three main respects from their approach. First, they consider only a dichotomous skill distribution and thus neglect addressing recent observations regarding polarizing wage trends. Second, by accounting for endogenous choice between different production techniques, the results of the present analysis reveal that a strength of the direct market size effect, as highlighted by Acemoglu et al. (2012), will eventually vanish as the degree of technology adoption becomes very high. Finally, differently from Acemoglu et al. (2012), the framework of the present paper allows to compare the implications of immigration and offshore for native workers regarding changes in the composition of jobs and tasks.

Third, this paper is also related to the literature examining the role of firm heterogeneity in conjunction with distributional effects of offshoring. In a two-country model with firm heterogeneity à la Melitz (2003), Egger et al. (2013) analyze the welfare and distributional impacts of offshoring. They show that offshoring generates a non-monotonic income distribution effect in the share of offshoring firms. The intuition for this relationship is that at initial high levels of variable offshoring costs, the share of offshoring firms is low, and a marginal decline in offshoring cost induces an unfavorable shift of domestic labor from more to low productive firms, inducing a deteriorating welfare effect (in terms of income per capita). This result highlights the efficiency gains at the intensive margin, while instead this framework accounts for efficiency gains the the extensive margin. However, the important departure of the present analysis from their framework is marked by the assumption of skill heterogeneity. This allows to address the direct and indirect implications of globalization for various skill groups - a crucial feature that is ignored in

\footnotetext{
${ }^{5}$ Rodríguez-Clare (2010) examines the offshoring-induced interaction between productivity and termof-trade effects in a two-country model. He shows that higher fragmentation shifts workers to the research sector. This effect leads, in the short-run, to a decline in the real wages due to a dominating negative terms-of-trade effect for the offshoring country, while, in the long run, wages rise due to higher productivity gains in the wake of higher stock of ideas per worker. Moreover, immigration induces a net gain effect only for immigrants, since they earn now higher wages in the offshoring country, while offshoring generates benefits for firms in offshoring countries due to lower unit costs.

${ }^{6}$ Other recent contributions studying the wage effects of offshoring are Baldwin and Robert-Nicoud (2014); Costinot and Vogel (2010).
} 
their framework.

Finally, this paper contributes also to the growing literature on the role of task content of jobs and the determinants of wage polarization. A number of studies has emphasized the key role of information and communication technology (ICT) behind the recent wage polarization (cf. Autor and Dorn, 2013; Autor et al., 2006; Michaels et al., 2014). Another set of studies provide a competing explanation by emphasizing the role of offshoring domestic jobs as the key determinant behind this trend (cf. Goos and Manning, 2007; Goos et al., 2014; Vallizadeh et al., 2015). While the empirical evidence is at odds, there is, however, a widespread consensus that jobs in the middle range of skill distribution are mostly prone to offshoring or to be substitute by technology - a feature that is also captured in this framework. Furthermore, recent empirical evidence has highlighted the differential specialization pattern between immigrants and natives in complementing tasks, e.g. in manual and communication intensive tasks, respectively (cf. Peri and Sparber, 2009). In this sense, this paper integrates different strands in the literature into a unified general equilibrium framework.

\subsection{The Model}

This section provides the description of the basic environment of the economy, characterizing the structure the goods market, the production technology, resource allocation and the adoption of different production technology modes. ${ }^{7}$ The following sections, then, elaborate the formal details of the extension with respect to task allocation between immigrants and natives and to offshoring opportunities.

\subsubsection{Basic environment}

The economy is described by a continuum of final goods $y(z)$ combined over the unit interval according to the following CES production function to produce a final consumption aggregate

$$
Y=\left[\int_{0}^{z_{L}} y_{L}(z)^{\frac{\sigma-1}{\sigma}} \mathrm{d} z+\int_{z_{L}}^{z_{H}} y_{M}(z)^{\frac{\sigma-1}{\sigma}} \mathrm{d} z+\int_{z_{H}}^{1} y_{H}(z)^{\frac{\sigma-1}{\sigma}} \mathrm{d} z\right]^{\frac{\sigma}{\sigma-1}}
$$

The final goods are indexed $z \in[0,1]$ in increasing order of production technology productivity, and $\sigma \geq 1$ denotes the elasticity of substitution between final good varieties. Then, by means of profit maximization subject to Eq. (5.1), the demand for any final good variety $z$ is given by

$$
y_{k}(z)=Y p_{k}(z)^{-\sigma}, \text { for } k=\{L, M, H\}
$$

where the price of aggregate consumption good is set to unity, i.e. $P_{Y}=1$.

\footnotetext{
${ }^{7}$ In chapter 4, a detailed analytical discussions of this model economy and its equilibrium characteristics is provided. Thus, in what follows allocation, formal details are deliberately kept implicit for the stake of convenience, but whenever necessary they are made explicit.
} 


\section{Final goods market}

The final goods market is perfectly competitive and each final good $z$ can be produced in three different technology modes: low- $\left(\varphi_{L}(z)\right)$, medium- $\left(\varphi_{M}(z)\right)$, and high-quality $\left(\varphi_{H}(z)\right)$ techniques. The choice of technology mode is based on the Ricardian type of comparative advantages, where final good firms self-select endogenously into the most cost-efficient production technique. As discussed in chapter 4, the equilibrium is characterized by two endogenous final goods margins, $z_{L}$ and $z_{H}$, where final good firms are indifferent between using low- and medium-quality techniques and between mediumand high-quality techniques, respectively. These conditions are given by

$$
\begin{aligned}
& \frac{P_{L}}{P_{M}}=\Lambda_{L}\left(z_{L}\right), \\
& \frac{P_{M}}{P_{H}}=\Lambda_{H}\left(z_{H}\right),
\end{aligned}
$$

where $\Lambda_{L}\left(z_{L}\right) \equiv \frac{\varphi_{L}\left(z_{L}\right)}{\varphi_{M}\left(z_{L}\right)}$ and $\Lambda_{H}\left(z_{H}\right) \equiv \frac{\varphi_{M}\left(z_{H}\right)}{\varphi_{H}\left(z_{H}\right)}$, and both are continuous and decreasing in their arguments, see chapter 4 for formal details and discussion.

It follows that each technology mode is used to produce a distinct range of final goods

$$
y(z)=\left\{\begin{array}{lll}
\varphi_{L}(z) X_{L} & \text { for all } z \in\left[0, z_{L}\right], \\
\varphi_{M}(z) X_{M} & \text { for all } \quad z \in\left(z_{L}, z_{H}\right), \\
\varphi_{H}(z) X_{H} & \text { for all } z \in\left[z_{H}, 1\right] .
\end{array}\right.
$$

where $X_{k}$ is a composite of intermediate goods, for $k=\{L, M, H\}$. The respective price schedule is given by

$$
p(z)= \begin{cases}P_{L} \varphi_{L}(z)^{-1} & \text { for all } z \in\left[0, z_{L}\right], \\ P_{M} \varphi_{M}(z)^{-1} & \text { for all } z \in\left(z_{L}, z_{H}\right), \\ P_{H} \varphi_{H}(z)^{-1} & \text { for all } z \in\left[z_{H}, 1\right],\end{cases}
$$

where $P_{k}$ denotes the effective price index of final goods, i.e. the price per productive technology $k$, which will be defined below.

\section{Intermediate goods market}

In contrast to the final goods market, the intermediate goods market is characterized by monopolistic competition, where each intermediate, in turn, is manufactured by labor of skill $k$, denoted by $\tilde{L}_{k}(j)$ for variety $j$. Moreover, each intermediate variety requires a fixed cost (measured in terms of the numeraire good), where the size depends on the adopted technology mode, such that $f_{L}<f_{M}<f_{H}$. The production technology of the composite good $X_{k}$ is represented by a CES aggregator over a continuum of intermediate varieties, indexed by $j$ :

$$
X_{k}=\left(\int_{0}^{A_{k}} x_{k}(j)^{\frac{\epsilon-1}{\epsilon}} \mathrm{d} j\right)^{\frac{\epsilon}{\epsilon-1}},
$$

where $x_{k}(j)$ denotes the amount of intermediate variety $j \in\left[0, A_{k}\right], \epsilon \geq 1$ captures the elasticity of substitution between varieties, and $A_{k}$ measures the mass of monopolists in 
technology mode $k^{8}$

By means of cost minimization subject to Eq. (5.7), the inverse demand function for intermediate good $j$ is given by:

$$
p_{k}(j)=\zeta_{k} X_{k}^{\frac{1}{\epsilon}} x_{k}(j)^{-\frac{1}{\epsilon}}, \forall k=\{L, M, H\},
$$

where $p_{k}(j)$ is the price of intermediate variety $j$ produced with technology mode $k$. Combining (5.8) with (5.7) yields the marginal cost of composite intermediate goods

$$
\zeta_{k}=\left(\int_{0}^{A_{k}} p_{k}(j)^{1-\epsilon} \mathrm{d} j\right)^{\frac{1}{1-\epsilon}}
$$

Under the constant elastic demand function (5.8) of degree $\epsilon>1$, the profit maximizing monopolist sets the price equal to a markup $\epsilon /(\epsilon-1)$ over the respective marginal cost, i.e.

$$
p_{k}(j)=\frac{\epsilon}{\epsilon-1} \tilde{w}_{k},
$$

where $\tilde{w}_{k}$ denotes the marginal cost of $k$-type factor labor, defined below.

\subsubsection{Task allocation between immigrants and natives}

As discussed above, recent empirical findings have highlighted the differential comparative advantages in job task performance between immigrants and natives, where immigrants tend to perform on average low communicative type of tasks and natives tend to specialize in communication- and complexity-intensive tasks (Ottaviano et al., 2013; Peri, 2012; Peri and Sparber, 2009). This has been put forth as the key rationale behind the imperfect substitutability between immigrants and natives, inducing a productivity effect for native workers associated with the efficient pattern of task specialization. Building on Grossman and Rossi-Hansberg (2008), a task assignment model is designed that is characterized by a continuum of tasks over the unit interval. While natives in each skill group $k$ are homogeneous in terms of their communication abilities, the task productivity among immigrants is more heterogeneous, leading to specialization of immigrants in low communicative tasks.

More precisely, the labor input $\tilde{L}_{k}(j)$ utilized by each intermediate variety $x_{k}(j)$, is now characterized by composite of a continuum of tasks, indexed $i \in[0,1]$ in increasing order of communication, inter-personal intensity. If a task $i$ is assigned to immigrants $(d=F)$, its input requirement is $\gamma_{k}(i) \geq 1$, where $\gamma_{k}(i)$ is continuously increasing in $i$. The marginal cost associated with tasked performed by immigrants is denoted by $w_{k}^{F}$. The input requirement using natives $(d=D)$ is 1 , with marginal cost $w_{k}^{D}$. Following the recent empirical studies highlighting the increasing importance of interactive, communicationintensive tasks in workplaces in the modern economy (cf. Borghans et al., 2008, 2013;

\footnotetext{
${ }^{8}$ For a discussion on the degree of external increasing returns to scales and the relationship between composite intermediate good $X_{k}$ and mass of monopolists $A_{k}$, see chapter 4 .
} 
Spitz-Oener, 2006; Stasz, 1997), I assume that the compensation of communicationintensive tasks is higher relative to non-communication tasks, i.e. $w_{k}^{D}>w_{k}^{F}$ for all $k=\{L, M, H\}$. An intermediate-goods producer $j \in\left[0, A_{k}\right]$ will assign tasks to immigrants up to an endogenously determined threshold $I_{k}$, where $I_{k} \in(0,1)$, so that the composite labor input is produced according to the following CES technology:

$$
\tilde{L}_{k}(j)=\left(\int_{0}^{I_{k}}\left(\frac{l_{k}^{F}(i)}{\gamma_{k}(i)}\right)^{\frac{\alpha-1}{\alpha}} \mathrm{d} i+\int_{I_{k}}^{1}\left(l_{k}^{D}(i)\right)^{\frac{\alpha-1}{\alpha}} \mathrm{d} i\right)^{\frac{\alpha}{\alpha-1}}
$$

where $\alpha \geq 1$ denotes the elasticity of substitution between tasks. The following lemma establishes the optimal conditions regarding the fraction of tasks performed by immigrants and natives

Lemma 5.1. Let $L_{k}^{D}$ and $L_{k}^{F}$ denote the amount of native and immigrant workers, respectively, which are employed by intermediate-good firm $j \in\left[0, A_{k}\right]$, for $k=\{L, M, H\}$, to perform a range of tasks. Then, the equilibrium unit factor labor is characterized by

$$
\tilde{L}_{k}(j)=\left(\vartheta_{k}\left(I_{k}\right)^{\frac{1}{\alpha}}\left(L_{k}^{F}\right)^{\frac{\alpha-1}{\alpha}}+\left[1-I_{k}\right]^{\frac{1}{\alpha}}\left(L_{k}^{D}\right)^{\frac{\alpha-1}{\alpha}}\right)^{\frac{\alpha}{\alpha-1}},
$$

where $\vartheta_{k}\left(I_{k}\right) \equiv\left(\int_{0}^{I_{k}} \gamma_{k}(i)^{1-\alpha} \mathrm{d} i\right)$, and the optimality condition for the marginal task $I_{k}$ is denoted by

$$
w_{k}^{D}=w_{k}^{F} \gamma_{k}\left(I_{k}\right)
$$

Proof. See Appendix 5.A.1.

Having defined the structure of the economy and the optimality conditions, I proceed now with the discussion of the characteristics of the equilibrium followed by the comparative statics.

\subsection{Characteristics of equilibrium}

The characteristics of the equilibrium are similar to chapter 4, except for the labor market clearing conditions and related variables, which require now the market clearing for both immigrants and natives in every skill group. The equilibrium in intermediate goods market is characterized by the standard symmetry assumption, where all firms are identical in the sense that the varieties of the differentiated good $X_{k}$ employ the same amount of factor labor $\tilde{L}_{k}(j)=\tilde{L}_{k}$, are sold at the same price $p_{k}(j)=p_{k}$ and are produced in equal amount $x_{k}(j)=x_{k}$, for all $k=\{L, M, H\}$.

Free entry: Free entry ensures that each intermediate-good firm makes zero profits so that the quantity for each variety is defined by

$$
x_{k}=(\epsilon-1) f_{k}, \quad \forall \quad k=\{L, M, H\} .
$$

Marginal costs of intermediate goods and price index: Next, using the monopolistic pricing 
behavior, Eq. (5.10), the marginal cost of the differentiated good $\zeta_{k}$, defined in Eq. (5.9), simplifies to

$$
\zeta_{k}=A_{k}^{\frac{1}{1-\epsilon}} p_{k}=\frac{\epsilon}{\epsilon-1} A_{k}^{\frac{1}{1-\epsilon}} \tilde{w}_{k}, \quad \forall k=\{L, M, H\},
$$

where the marginal cost is defined by $\tilde{w}_{k}=\left(\vartheta_{k}\left(I_{k}\right)\left(w_{k}^{F}\right)^{1-\alpha}+\left[1-I_{k}\right]\left(w_{k}^{D}\right)^{1-\alpha}\right)^{\frac{1}{1-\alpha}}$ (see Appendix 5.A.1 for a formal discussion).

The perfectly competitive nature of the final-goods market requires that any final good producer makes zero profits, i.e. $p_{k}(z) y_{k}(z)=\zeta_{k} X_{k}$, for all $k=\{L, M, H\}$. Utilizing Eqs. (5.5) and (5.6), it follows that the price index of technology $k$ must equal the marginal cost, i.e.

$$
P_{k}=\zeta_{k}
$$

Resource constraint: Let the total amount of labor used in each set of technology $\left[0, A_{k}\right]$ be denoted by $\int_{0}^{A_{k}} L_{k}^{d} \mathrm{~d} j=n_{k}^{d}$, for $d=\{F, D\}$, and the total resource constraint (i.e. over the range of final goods produced with technology mode $k$ ) by $\int_{z \in \mathcal{S}_{k}} n_{k}^{d} \mathrm{~d} z=N_{k}^{d}$, where $\mathcal{S}_{L}=\left\{0, z_{L}\right\}, \mathcal{S}_{M}=\left\{z_{L}, z_{H}\right\}$, and $\mathcal{S}_{H}=\left\{z_{H}, 1\right\}$ and $N_{L}^{d}, N_{M}^{d}$, and $N_{H}^{d}$ are the total (exogenously given) endowments of labor. Then, by symmetry it follows

$$
\begin{gathered}
L_{L}^{F}=\frac{N_{L}^{F}}{z_{L} A_{L}}, \quad L_{L}^{D}=\frac{N_{L}^{D}}{z_{L} A_{L}}, \\
L_{M}^{F}=\frac{N_{M}^{F}}{\left(z_{H}-z_{L}\right) A_{M}}, \quad L_{M}^{D}=\frac{N_{M}^{D}}{\left(z_{H}-z_{L}\right) A_{M}}, \\
L_{H}^{F}=\frac{N_{H}^{F}}{\left(1-z_{H}\right) A_{H}}, \quad L_{H}^{D}=\frac{N_{H}^{D}}{\left(1-z_{H}\right) A_{H}} .
\end{gathered}
$$

Composite intermediate output: Next, combining the labor market conditions (5.17), (5.18), and (5.19), with Eq. (5.12) yields the equilibrium value of intermediate output

$$
x_{L}=\frac{\tilde{N}_{L}}{z_{L} A_{L}}, \quad x_{M}=\frac{\tilde{N}_{M}}{\left(z_{H}-z_{L}\right) A_{M}}, \quad x_{H}=\frac{\tilde{N}_{H}}{\left(1-z_{H}\right) A_{H}},
$$

where $\tilde{N}_{k} \equiv\left(\vartheta_{k}\left(I_{k}\right)^{\frac{1}{\alpha}}\left(N_{k}^{F}\right)^{\frac{\alpha-1}{\alpha}}+\left[1-I_{k}\right]^{\frac{1}{\alpha}}\left(N_{k}^{D}\right)^{\frac{\alpha-1}{\alpha}}\right)^{\frac{\alpha}{\alpha-1}}$, for all $k=\{L, M, H\}$.

Substituting the results from (5.20) into the composite intermediate goods function (5.7) for the respective technology mode, we get

$$
X_{L}=A_{L}^{\frac{1}{\epsilon-1}} \frac{\tilde{N}_{L}}{z_{L}}, \quad X_{M}=A_{M}^{\frac{1}{\epsilon-1}} \frac{\tilde{N}_{M}}{z_{H}-z_{L}}, \quad X_{H}=A_{H}^{\frac{1}{\epsilon-1}} \frac{\tilde{N}_{H}}{1-z_{H}} .
$$

Mass of monopolists: Combining the budget constraint for any final good producer $z$,

$$
p(z) y(z)=w_{k}^{F} \int_{0}^{A_{k}} L_{k}^{F} \mathrm{~d} j+w_{k}^{D} \int_{0}^{A_{k}} L_{k}^{D} \mathrm{~d} j,
$$


with Eqs. (5.5), (5.6), (5.7), (5.14) and the resource constraints (5.17) (5.18), and (5.19), then the mass of monopolists in each technology mode $k$ can be pinned down to the skill endowment, fixed costs, the range of final goods, and the market power of monopolists (see Appendix 5.B.1 for details of the formal derivation).

$$
A_{L}=\frac{\tilde{N}_{L}}{\epsilon f_{L} z_{L}}, \quad A_{M}=\frac{\tilde{N}_{M}}{\epsilon f_{M}\left(z_{H}-z_{L}\right)}, \quad A_{H}=\frac{\tilde{N}_{H}}{\epsilon f_{H}\left(1-z_{H}\right)} .
$$

An inspection of eq. (5.22) reveals important properties of specialization and increasing returns to scale. It is easily seen that for given values of $z_{L}$ and $z_{H}$, changes in the labor endowment $\tilde{N}_{k}$ leads to an increase in the mass of monopolists. This reflects the direct market-size effect, capturing the notion of directed technological change elicited by higher profitability of producing intermediate goods $x_{k}$. This in turn raises the comparative advantage of technology mode $k$, leading to an expansion in the range of final goods produced by the complementing technology mode $k$ and thus to changes in $z_{L}$ and $z_{H}$. This effect reflects the aforementioned technology adoption effect. However, this latter effect counteracts the former one in the wake of a price effect, favoring the competing technology modes. This interaction is analog to the idea of internal and external increasing returns to scale forces in the context of international trade as in Ethier (1982). Moreover, this pattern of specialization gives rise to increasing returns to scale at aggregate industry level - similar to semi-endogenous growth models as in Romer (1987).

Labor composite and marginal costs: There exists within-skill group heterogeneity due to differential task productivity between immigrants and natives. The convenient block recursive structure of the model allows to solve first for the equilibrium task margin $I_{k}$ and simplify the labor composite and the marginal cost of factor labor as expressions of the task margin as well as of natives endowments and wages, respectively. In doing so, utilize Lemma 5.1 and labor market clearing conditions (5.18) to rewrite the composite factor labor and marginal cost of every intermediate-good producer using technology mode $k$. Given the equilibrium task margin $I_{k}>0$, it follows (see Appendix 5.A.2)

$$
\begin{aligned}
& \tilde{N}_{k}=\Theta_{k}\left(I_{k}\right)^{-\alpha} \frac{N_{k}^{D}}{1-I_{k}}, \\
& \tilde{w}_{k}=\Theta_{k}\left(I_{k}\right) w_{k}^{D},
\end{aligned}
$$

where the generalized measure of marginal cost of tasks is denoted by

$$
\Theta_{k}\left(I_{k}\right) \equiv\left[\vartheta_{k}\left(I_{k}\right) \gamma_{k}\left(I_{k}\right)^{\alpha-1}+1-I_{k}\right]^{\frac{1}{1-\alpha}}<1
$$

These results indicate the key feature of the task-based approach characterizing the productivity gains due to task specialization patterns between immigrants and natives. The following lemma summarizes this result.

Lemma 5.2. For $I_{k}>0$, an increase in $k$-skill endowment of immigrants raises the task margin, i.e.

$$
\frac{\mathrm{d} \ln I_{k}}{\mathrm{~d} \ln N_{k}^{F}}>0
$$


which in turn induces a cost-efficiency enhancing effect, associated with lower marginal costs, i.e.

$$
\frac{\mathrm{d} \ln \Theta_{k}\left(I_{k}\right)}{\mathrm{d} \ln I_{k}}<0,
$$

and an increase in the composite labor, i.e.

$$
\frac{\mathrm{d} \ln \tilde{N}_{k}}{\mathrm{~d} \ln I_{k}}=-\frac{\mathrm{d} \ln \left[\Theta_{k}\left(I_{k}\right)^{\alpha}\left(1-I_{k}\right)\right]}{\mathrm{d} \ln I_{k}}>0
$$

Proof. See Appendix 5.A.3.

Thus, the results in Lemma 5.2 highlight the important feature of the task-assignment approach, whereby immigration gives rise to efficiency gains through specialization. This is a crucial departure from models that account only implicitly for immigrants (in terms of marginal changes in the endowment), or in a static manner through a CES-aggregator. Instead, immigration is now associated with an endogenous response by native workers, which has been put forth as the salient feature regarding the efficiency improvement, the so-called productivity effect. This effect is captured by $\Theta_{k}\left(I_{k}\right)$ in Eq. (5.25).

Final goods: Utilizing Eqs. (5.5) and (5.21), the production function of final goods can be then rewritten as

$$
y(z)= \begin{cases}\varphi_{L}(z) X_{L}=\varphi_{L}(z) A_{L}^{\frac{1}{\epsilon-1}} \frac{\tilde{N}_{L}}{z_{L}}, & \text { for all } z \in\left[0, z_{L}\right], \\ \varphi_{M}(z) X_{M}=\varphi_{M}(z) A_{M}^{\frac{1}{\epsilon-1}} \frac{\tilde{N}_{M}}{z_{H}-z_{L}}, & \text { for all } z \in\left(z_{L}, z_{H}\right), \\ \varphi_{H}(z) X_{H}=\varphi_{H}(z) A_{H}^{\frac{1}{\epsilon-1}} \frac{\tilde{N}_{H}}{1-z_{H}}, & \text { for all } z \in\left[z_{H}, 1\right],\end{cases}
$$

Aggregate output by technology modes: Finally, we derive the equilibrium aggregate levels of output and expenditure for each technology mode $k$. Let the total cost using technology $k$ be

$$
P_{k} Y_{k}=\int_{z \in \mathcal{S}_{k}} p(z) y(z) \mathrm{d} z,
$$

where $Y_{k}$ denotes the aggregate level of output of final goods using technology mode $k$. Now, utilizing Eqs. (5.6) and (5.26) in the previously derived equation and manipulating slightly, yields the aggregate level of output per technology mode $k$ (see Appendix 5.B.2 for the formal derivation)

$$
Y_{L}=A_{L}^{\frac{1}{\epsilon-1}} \tilde{N}_{L}, \quad Y_{M}=A_{M}^{\frac{1}{\epsilon-1}} \tilde{N}_{M}, \quad Y_{H}=A_{H}^{\frac{1}{\epsilon-1}} \tilde{N}_{H}
$$

Relative expenditure on technology modes: As shown in chapter 4, it follows from Eq. (5.2) that the expenditure on final goods must equal at the equilibrium technology margins, $z_{L}$ and $z_{H}$. Utilizing Eqs. (5.21), (5.22), and (5.23), then we obtain the expression for 
the relative price index of final goods produced with technology mode $k=M$

$$
\begin{aligned}
\frac{P_{L}}{P_{M}} & =\left(\frac{f_{L}}{f_{M}}\right)^{\frac{1}{(\epsilon-1)}}\left(\frac{\mathcal{M}\left(I_{M}\right)}{\mathcal{M}\left(I_{L}\right)}\right)^{\frac{\epsilon}{(1-\epsilon)}}\left(\frac{N_{M}^{D}}{N_{L}^{D}}\right)^{\frac{\epsilon}{(\epsilon-1)}}\left(\frac{z_{L}}{z_{H}-z_{L}}\right)^{\frac{\epsilon}{(\epsilon-1)}}, \\
\frac{P_{M}}{P_{H}} & =\left(\frac{f_{M}}{f_{H}}\right)^{\frac{1}{(\epsilon-1)}}\left(\frac{\mathcal{M}_{H}\left(I_{H}\right)}{\mathcal{M}_{M}\left(I_{M}\right)}\right)^{\frac{\epsilon}{(1-\epsilon)}}\left(\frac{N_{H}^{D}}{N_{M}^{D}}\right)^{\frac{\epsilon}{(\epsilon-1)}}\left(\frac{z_{H}-z_{L}}{1-z_{H}}\right)^{\frac{\epsilon}{(\epsilon-1)}} .
\end{aligned}
$$

The variable $\mathcal{M}_{k}\left(I_{k}\right) \equiv \Theta_{k}\left(I_{k}\right)^{\alpha}\left(1-I_{k}\right)$ measures for all $k=\{L, M, H\}$ the impact of migration associated with endogenous changes in the task margin, as shown in Lemma 5.2. It is decreasing in $I_{k}$, implying that the labor composite is increasing in $I_{k}$, i.e. $\frac{\mathrm{d} \ln \tilde{N}_{k}}{\mathrm{~d} \ln I_{k}}=-\frac{\mathrm{d} \ln \mathcal{M}_{k}\left(I_{k}\right)}{\mathrm{d} \ln I_{k}}>0$ (see again Appendix 5.A.3). It is worth mentioning the crucial differences to the case of implicit immigration discussed in chapter 4. Evaluating the functional properties of $\mathcal{M}_{k}\left(I_{k}\right)$, it can be shown that $\lim _{I_{k} \rightarrow 0} \frac{\mathrm{d} \ln \tilde{N}_{k}}{\mathrm{~d} \ln I_{k}}=-\frac{\mathrm{d} \ln \mathcal{M}_{k}\left(I_{k}\right)}{\mathrm{d} \ln I_{k}}=0$ and $\lim _{I_{k} \rightarrow 1} \frac{\mathrm{d} \ln \tilde{N}_{k}}{\mathrm{~d} \ln I_{k}}=-\frac{\mathrm{d} \ln \mathcal{M}_{k}\left(I_{k}\right)}{\mathrm{d} \ln I_{k}}=\infty$. This indicates the efficiency effect of immigration: an increase in $I_{k}$ induces an cost-efficiency enhancing effect through specialization, captured by the reallocation of tasks between natives and immigrants.

To close the model, combine (5.28) and (5.29) with the efficient allocation of technology modes, Eqs. (5.3) and (5.4), respectively. Now taking logs, multiplying through $(\epsilon-1) / \epsilon$, and rearranging slightly, we obtain two implicit functions $\mathcal{F}_{L}(\cdot)$ and $\mathcal{F}_{H}(\cdot)$ that define the equilibrium technology margins $z_{L}$ and $z_{H}$ as functions of parameters and variables of the model.

$$
\begin{aligned}
\mathcal{F}_{L}\left(z_{L}, z_{H}, f_{L}, f_{M}, I_{L}, I_{M}\right) \equiv & \frac{1}{\epsilon} \ln \left(\frac{f_{L}}{f_{M}}\right)+\ln \left(\frac{\mathcal{M}_{M}\left(I_{M}\right)}{\mathcal{M}_{L}\left(I_{L}\right)}\right)+\ln \left(\frac{N_{M}^{D}}{N_{L}^{D}}\right)+\ln \left(\frac{z_{L}}{z_{H}-z_{L}}\right) \\
& -\frac{(\epsilon-1)}{\epsilon} \ln \Lambda_{L}\left(z_{L}\right)=0, \\
\mathcal{F}_{H}\left(z_{L}, z_{H}, f_{H}, f_{M}, I_{H}, I_{M}\right) \equiv & \frac{1}{\epsilon} \ln \left(\frac{f_{M}}{f_{H}}\right)+\ln \left(\frac{\mathcal{M}_{H}\left(I_{H}\right)}{\mathcal{M}_{M}\left(I_{M}\right)}\right)+\ln \left(\frac{N_{H}^{D}}{N_{M}^{D}}\right)+\ln \left(\frac{z_{H}-z_{L}}{1-z_{H}}\right) \\
& -\frac{(\epsilon-1)}{\epsilon} \ln \Lambda_{H}\left(z_{H}\right)=0 .
\end{aligned}
$$

This implicit system of equations can be used to compute basic comparative statics of changes in factor endowment and fixed market entry costs and their implications for technology adoption.

\subsection{Comparative statics}

\subsubsection{Immigration and technology adoption}

Recall from Lemma 5.2 that immigration is associated with a positive shift in the task margin $I_{k}$. For the computation of the comparative statics it is therefore sufficient to consider changes in the task margin. This implies that all the results derived here are akin to those derived in chapter 4 , except that now the relationship between the technology 
margins, $z_{L}$ and $z_{H}$, and the task margin $I_{k}$ is affected by the initial level of task margin. To get a better idea, differentiate the implicit Eqs. (5.30) and (5.31) with respect to $I_{M}$ and the margins $\left(z_{L}\right.$ and $\left.z_{H}\right)$ to obtain

$$
\begin{aligned}
\left(\begin{array}{ll}
\frac{\partial \mathcal{F}_{L}}{\partial z_{L}} & \frac{\partial \mathcal{F}_{L}}{\partial z_{H}} \\
\frac{\partial \mathcal{F}_{H}}{\partial z_{L}} & \frac{\partial \mathcal{F}_{H}}{\partial z_{H}}
\end{array}\right)\left(\begin{array}{l}
\mathrm{d} \ln z_{L} \\
\mathrm{~d} \ln z_{H}
\end{array}\right) & =\left(\begin{array}{c}
-\frac{\partial \mathcal{F}_{L}}{\partial I_{M}} \\
-\frac{\partial \mathcal{F}_{H}}{\partial I_{M}}
\end{array}\right) \times \mathrm{d} \ln I_{M}, \\
\Leftrightarrow\left(\begin{array}{cc}
\frac{\epsilon-1}{\epsilon} \tilde{\varepsilon}_{L} z_{L}+\frac{z_{H}}{z_{H}-z_{L}} & -\frac{z_{H}}{z_{H}-z_{L}} \\
-\frac{z_{L}}{z_{H}-z_{L}} & \frac{\epsilon-1}{\epsilon} \tilde{\varepsilon}_{H} z_{H}+\frac{z_{H}\left(1-z_{L}\right)}{\left(1-z_{H}\right)\left(z_{H}-z_{L}\right)}
\end{array}\right)\left(\begin{array}{l}
\mathrm{d} \ln z_{L} \\
\mathrm{~d} \ln z_{H}
\end{array}\right) & =\left(\begin{array}{c}
-\Psi_{M} \\
\Psi_{M}
\end{array}\right) \times \mathrm{d} \ln I_{M},
\end{aligned}
$$

where $\tilde{\varepsilon}_{k} \equiv-\frac{\partial \ln \Lambda_{k}\left(z_{k}\right)}{\partial \ln z_{k}}>0$, for $k=\{L, H\}$ denotes the elasticity (in absolute value) of relative efficiency schedules of technology modes $k$ at the equilibrium margin $z_{k}$ and $\Psi_{M} \equiv-\frac{\mathrm{d} \ln \mathcal{M}_{M}\left(I_{M}\right)}{\mathrm{d} \ln I_{M}}>0$. The inspection of the matrix verifies that the Jacobi has a positive sign, i.e. $\mathcal{D}_{J}=\frac{\partial \mathcal{F}_{L}}{\partial z_{L}} \frac{\partial \mathcal{F}_{H}}{\partial z_{H}}-\frac{\partial \mathcal{F}_{L}}{\partial z_{H}} \frac{\partial \mathcal{F}_{H}}{\partial z_{L}}>0$, and thus the solution to the above $2 \times 2$ system is unique. Then, by Cramer's rule, we obtain

$$
\frac{\mathrm{d} \ln z_{L}}{\mathrm{~d} \ln I_{M}}=\frac{\mathrm{d} \ln \mathcal{M}_{M}}{\mathrm{~d} \ln I_{M}} \frac{\mathrm{d} \ln z_{L}}{\mathrm{~d} \ln \mathcal{M}_{M}}=-\Psi_{M} \frac{\frac{z_{H}}{1-z_{H}}+\frac{(\epsilon-1)}{\epsilon} \tilde{\varepsilon}_{H} z_{H}}{\mathcal{D}_{J}}<0,
$$

and

$$
\frac{\mathrm{d} \ln z_{H}}{\mathrm{~d} \ln I_{M}}=\frac{\mathrm{d} \ln \mathcal{M}_{M}}{\mathrm{~d} \ln I_{M}} \frac{\mathrm{d} \ln z_{H}}{\mathrm{~d} \ln \mathcal{M}_{M}}=\Psi_{M} \frac{1+\frac{\epsilon-1}{\epsilon} \tilde{\varepsilon}_{L} z_{L}}{\mathcal{D}_{J}}>0 .
$$

The results of the comparative statics in Eqs. (5.32) and (5.33) highlight the convenient feature of the model. It allows to decompose immigration-induced changes in the technology margins, $z_{L}$ and $z_{H}$, into changes due to internal task reallocation between immigrants and natives, captured by $\Psi_{k}$, and changes in the technology margins due to endogenous technology adoption. The following proposition summarizes the main results.

Proposition 5.1. An exogenous increase in the skill endowment of immigrants and in the fixed costs will affect the technology margins $z_{L}$ and $z_{H}$ in the following way:

- Immigration of high-skill workers and $H$-fixed cost

$$
\left\{\frac{\mathrm{d} z_{L}}{\mathrm{~d} \ln I_{H}}, \frac{\mathrm{d} z_{H}}{\mathrm{~d} \ln I_{H}}\right\}<0,\left\{\frac{\mathrm{d} z_{L}}{\mathrm{~d} \ln f_{H}}, \frac{\mathrm{d} z_{H}}{\mathrm{~d} \ln f_{H}}\right\}>0
$$

- Immigration of medium-skilled workers, easier offshoring, and $M$-fixed cost

$$
\left\{\frac{\mathrm{d} z_{L}}{\mathrm{~d} \ln f_{M}}, \frac{\mathrm{d} z_{H}}{\mathrm{~d} \ln I_{M}}\right\}>0,\left\{\frac{\mathrm{d} z_{L}}{\mathrm{~d} \ln I_{M}}, \frac{\mathrm{d} z_{H}}{\mathrm{~d} \ln f_{M}}\right\}<0,
$$

- Immigration of low-skilled workers and L-fixed cost

$$
\left\{\frac{\mathrm{d} z_{L}}{\mathrm{~d} \ln I_{L}}, \frac{\mathrm{d} z_{H}}{\mathrm{~d} \ln I_{L}}\right\}>0,\left\{\frac{\mathrm{d} z_{L}}{\mathrm{~d} \ln f_{L}}, \frac{\mathrm{d} z_{H}}{\mathrm{~d} \ln f_{L}}\right\}<0 .
$$

Proof. See Appendix 5.A.4 and the discussion in chapter 4. 
The intuition behind the marginal impact of task threshold $I_{k}$ on thresholds $z_{L}$ and $z_{H}$ differs from chapter 4 and deserves some discussions. As shown in Lemma 5.2, immigration leads to a reallocation of tasks from natives to immigrants (i.e. increase in $I_{k}$ ). This induces on the one hand an efficiency gains through specialization. On the other hand, it increases effectively natives labor supply of similar skill level. Both effects raise the skill endowment and thus lead to a larger factor market that allows to produce more varieties. The Walrasian nature of the labor market indicates a negative relationship between factor market size and factor prices. This in turn implies that firms are able to produce varieties at lower unit costs. Consequently, the comparative advantage and thus the adoption of the skill-complementing technology mode is increased.

Equipped with comparative statics for technology adoption effect, the next section computes the comparative statics of different immigration scenarios and changes in native wage structure.

\subsubsection{Immigration and native wage structure}

In equilibrium, the optimal demand condition for intermediate goods, Eq. (5.8), and the monopolistic price-setting condition, Eq. (5.10), are

$$
\frac{\epsilon}{\epsilon-1} \tilde{w}_{k}=p_{k}=\zeta_{k} X_{k}^{1 / \epsilon} x_{k}^{-1 / \epsilon}, \quad \forall k=\{L, M, H\} .
$$

Utilizing the equilibrium conditions (5.16), (5.20), (5.21), (5.22), (5.24), (5.27), into Eq. (5.34) and taking the ration between low- and medium-skilled and between medium- and high-skilled wages we obtain (see Appendix 5.B.3 for a full formal discussion):

$$
\begin{aligned}
\frac{w_{L}^{D}}{w_{M}^{D}} & =\left(\frac{z_{L}}{z_{H}-z_{L}}\right)^{\frac{\epsilon-\sigma}{(\epsilon-1) \sigma}}\left(\frac{\mathcal{M}_{L}\left(I_{L}\right)}{\mathcal{M}_{M}\left(I_{M}\right)}\right)^{\frac{(\epsilon-\sigma)}{(\epsilon-1) \sigma}}\left(\frac{\Theta_{L}\left(I_{L}\right)}{\Theta_{M}\left(I_{M}\right)}\right)^{-1} \mathcal{K}_{L M} \\
\frac{w_{M}^{D}}{w_{H}^{D}} & =\left(\frac{z_{H}-z_{L}}{1-z_{H}}\right)^{\frac{\epsilon-\sigma}{(-1) \sigma}}\left(\frac{\mathcal{M}_{M}\left(I_{M}\right)}{\mathcal{M}_{H}\left(I_{H}\right)}\right)^{\frac{(\epsilon-\sigma)}{(\epsilon-1) \sigma}}\left(\frac{\Theta_{M}\left(I_{M}\right)}{\Theta_{H}\left(I_{H}\right)}\right)^{-1} \mathcal{K}_{M H}
\end{aligned}
$$

where $\quad \mathcal{K}_{L M} \quad \equiv \quad\left(\frac{N_{L}^{D}}{N_{M}^{D}}\right)^{\frac{(\sigma-\epsilon)}{(\epsilon-1) \sigma}}\left(\frac{f_{L}}{f_{M}}\right)^{-\frac{\sigma-1}{(\epsilon-1) \sigma}} \Lambda_{L}\left(z, z^{\prime}\right) \quad$ and $\quad \mathcal{K}_{M H} \equiv$ $\left(\frac{N_{M}^{D}}{N_{H}^{D}}\right)^{\frac{(\sigma-\epsilon)}{(\epsilon-1) \sigma}}\left(\frac{f_{M}}{f_{H}}\right)^{-\frac{\sigma-1}{(\epsilon-1) \sigma}} \Lambda_{H}\left(z^{\prime}, z^{\prime \prime}\right)$ summarize the other exogenous variables.

An inspection of Eqs. (5.35) and (5.36) allows to decompose the impact of immigration on native wage structure into the following key channels: (i) a technology adoption effect, captured by $\left(\frac{z_{L}}{z_{H}-z_{L}}\right)^{\frac{\epsilon-\sigma}{(\epsilon-1) \sigma}}$ and $\left(\frac{z_{H}-z_{L}}{1-z_{H}}\right)^{\frac{\epsilon-\sigma}{(-1) \sigma}}$, indicating changes in the relative range of final goods; (ii) a market size effect, captured by $\left(\frac{\mathcal{M}_{L}\left(I_{L}\right)}{\mathcal{M}_{M}\left(I_{M}\right)}\right)^{\frac{(\epsilon-\sigma)}{(\epsilon-1) \sigma}}$ and $\left(\frac{\mathcal{M}_{M}\left(I_{M}\right)}{\mathcal{M}_{H}\left(I_{H}\right)}\right)^{\frac{(\epsilon-\sigma)}{(\epsilon-1) \sigma}}$, indicating changes in the skill endowment; and (iii) a productivity effect, captured by $\left(\Theta_{L}\left(I_{L}\right) / \Theta_{M}\left(I_{M}\right)\right)^{-1}$ and $\left(\Theta_{M}\left(I_{M}\right) / \Theta_{H}\left(I_{H}\right)\right)^{-1}$, associated with changes in the unit cost structure.

As highlighted in chapter 4, the technology adoption effect is interacting with the market 
size effect due to the the direct and indirect market size effects and the price effect induced by the market externalities mentioned earlier. Moreover, the direction of each of these effects depends on the strength of final goods demand elasticity, $\sigma$ relative to the market power of monopolists, $\epsilon$. To see how these two effect interact, let $\tilde{\Gamma}_{I_{k}} \equiv \frac{\mathrm{d} \ln \left(z_{L} /\left(z_{H}-z_{L}\right)\right)}{\mathrm{d} \ln I_{k}}$ and $\tilde{\Delta}_{I_{k}} \equiv \frac{\mathrm{d} \ln \left(\left(z_{H}-z_{L}\right) /\left(1-z_{H}\right)\right)}{\mathrm{d} \ln I_{k}}$ denote the marginal effect of immigration-induced changes in task margin $I_{k}$. As shown in Appendix 5.B.4, the convenient structure of the model allows to decompose this effect into the following terms:

$$
\tilde{\Gamma}_{I_{k}}=\Psi_{k} \Gamma_{k} \text { and } \quad \tilde{\Delta}_{I_{k}}=\Psi_{k} \Delta_{k}
$$

indicating a scalar product between the market size effect,

$$
\Psi_{k} \equiv-\frac{\mathrm{d} \ln \mathcal{M}_{k}}{\mathrm{~d} \ln I_{k}}=-\frac{\mathrm{d} \ln \left[\Theta_{k}\left(I_{k}\right)^{\alpha}\left(1-I_{k}\right)\right]}{\mathrm{d} \ln I_{k}}>0,
$$

and immigration-induced technology adoption effect, i.e.

$$
\Gamma_{k}=\frac{\mathrm{d} \ln \left(z_{L} /\left(z_{H}-z_{L}\right)\right)}{\mathrm{d} \ln \mathcal{M}_{k}} \quad \text { and } \quad \Delta_{k}=\frac{\mathrm{d} \ln \left(\left(z_{H}-z_{L}\right) /\left(1-z_{H}\right)\right)}{\mathrm{d} \ln \mathcal{M}_{k}} .
$$

The terms $\Gamma_{k}$ and $\Delta_{k}$ are akin to those computed in chapter 4 , and as discussed there changes in the neighborhood of the technology margins $z_{L}$ and $z_{H}$, depend on the degree of technology adoption, captured by the elasticity parameters $\tilde{\varepsilon}_{L} \equiv-\frac{\mathrm{d} \ln \Lambda_{L}\left(z_{L}\right)}{\mathrm{d} \ln z_{L}}>0$ and $\tilde{\varepsilon}_{H} \equiv-\frac{\mathrm{d} \ln \Lambda_{H}\left(z_{H}\right)}{\mathrm{d} \ln z_{H}}>0$, so that it follows that $\left\{\left|\Gamma_{k}\right|,\left|\Delta_{k}\right|\right\} \in(0,1)$, for $0<\left\{\tilde{\varepsilon}_{L}, \tilde{\varepsilon}_{H}\right\}<\infty$.

The rest of this section examines how the relative strength and the determinants of these different channels. In doing so, the impact of various skill immigration scenarios on native wage structure are intuitively discussed, while the formal derivation are relegated to Appendix 5.B.5. For ease of notation, let the relative wage of medium-skilled natives be $\omega_{L M}^{D} \equiv\left(w_{L}^{D} / w_{M}^{D}\right)$ and $\omega_{M H}^{D} \equiv\left(w_{M}^{D} / w_{H}^{D}\right)$.

\section{Low-skill immigration}

Now taking logs in Eqs. (5.35) and (5.36) and differentiating totally with respect to changes in the task margin $I_{L}$, and manipulating yields

$$
\begin{aligned}
\frac{\mathrm{d} \ln \omega_{L M}^{D}}{\mathrm{~d} \ln I_{L}} & =\left(1-\alpha\left(1-\Gamma_{L}\right) \frac{(\epsilon-\sigma)}{(\epsilon-1) \sigma}\right) \tilde{\Theta}_{L}\left(I_{L}\right)-\left(1-\Gamma_{L}\right) \frac{(\epsilon-\sigma)}{(\epsilon-1) \sigma} \frac{I_{L}}{1-I_{L}} \\
\frac{\mathrm{d} \ln \omega_{M H}^{D}}{\mathrm{~d} \ln I_{L}} & =\frac{\epsilon-\sigma}{(\epsilon-1) \sigma}\left(\tilde{\Theta}_{L}\left(I_{L}\right)+\frac{I_{L}}{1-I_{L}}\right) \Delta_{L}
\end{aligned}
$$

where $\tilde{\Theta}_{L}\left(I_{L}\right) \equiv-\frac{\mathrm{d} \ln \Theta_{L}\left(I_{L}\right)}{\mathrm{d} \ln I_{L}}>0$ denotes the productivity effect, and by Proposition 5.1 $\Gamma_{L}>0$ and $\Delta_{L}<0$. 


\section{Medium-skill immigration}

Following the same steps, the impact of high-skill immigration can be computed, i.e. taking logs in Eqs. (5.35) and (5.36) and differentiating totally with respect to changes in the task margin $I_{H}$, associated with high-skill immigration, yields

$$
\begin{aligned}
\frac{\mathrm{d} \ln \omega_{L M}^{D}}{\mathrm{~d} \ln I_{M}}= & -\left(1-\alpha\left(1+\Gamma_{M}\right) \frac{(\epsilon-\sigma)}{(\epsilon-1) \sigma}\right) \tilde{\Theta}_{M}\left(I_{M}\right) \\
& +\left(1+\Gamma_{M}\right) \frac{(\epsilon-\sigma)}{(\epsilon-1) \sigma} \frac{I_{M}}{1-I_{M}} \\
\frac{\mathrm{d} \ln \omega_{M H}^{D}}{\mathrm{~d} \ln I_{M}}= & \left(1-\alpha\left(1-\Delta_{M}\right) \frac{(\epsilon-\sigma)}{(\epsilon-1) \sigma}\right) \tilde{\Theta}_{M}\left(I_{M}\right) \\
& -\left(1-\Delta_{M}\right) \frac{(\epsilon-\sigma)}{(\epsilon-1) \sigma} \frac{I_{M}}{1-I_{M}},
\end{aligned}
$$

where $\tilde{\Theta}_{M}\left(I_{M}\right) \equiv-\frac{\mathrm{d} \ln \Theta_{M}\left(I_{M}\right)}{\mathrm{d} \ln I_{M}}>0$ denotes the productivity effect, by Proposition 5.1 , we get that $\Gamma_{M}<0$ and $\Delta_{M}>0$.

\section{High-skill immigration}

Following the same steps, the impact of high-skill immigration can be computed, i.e. taking logs in Eqs. (5.35) and (5.36) and differentiating totally with respect to changes in the task margin $I_{H}$ yields

$$
\begin{aligned}
\frac{\mathrm{d} \ln \omega_{L M}^{D}}{\mathrm{~d} \ln I_{H}}= & \frac{\epsilon-\sigma}{(\epsilon-1) \sigma}\left(\tilde{\Theta}_{H}\left(I_{H}\right)+\frac{I_{H}}{1-I_{H}}\right) \Gamma_{H} \\
\frac{\mathrm{d} \ln \omega_{M H}^{D}}{\mathrm{~d} \ln I_{H}}= & -\left(1-\alpha\left(1+\Delta_{H}\right) \frac{(\epsilon-\sigma)}{(\epsilon-1) \sigma}\right) \tilde{\Theta}_{H}\left(I_{H}\right) \\
& +\left(1+\Delta_{H}\right) \frac{(\epsilon-\sigma)}{(\epsilon-1) \sigma} \frac{I_{H}}{1-I_{H}},
\end{aligned}
$$

where $\tilde{\Theta}_{H}\left(I_{H}\right) \equiv-\frac{\mathrm{d} \ln \Theta_{H}\left(I_{H}\right)}{\mathrm{d} \ln I_{H}}>0$ denotes the productivity effect, and by Proposition 5.1 $\Gamma_{H}>0$ and $\Delta_{H}<0$.

Examination of Eqs (5.37)-(5.42) reveals the following new insights. First, allowing for internal adjustment of work organization, i.e. endogenous task specialization between immigrants and natives, immigration is associated with a productivity effect, $\tilde{\Theta}_{k}$, and a labor supply effect, $\frac{I_{k}}{1-I_{k}}$. Moreover, the relative strength between these two forces exhibits now a dynamic nature, varying with the initial level of task margin. As elaborated below, this novel feature of the model provides a rationale behind the ambiguity of the impact of immigration on native wage structure found often in the empirical literature.

In addition, the direction of each channel is importantly shaped by the degree of technology adoption between the different production modes, $\Gamma_{k}$ and $\Delta_{k}$, as well as by the relative strength between the final-goods demand elasticity $(\sigma)$ and the market power of monopolists $(\epsilon)$. However, it worth highlighting that the magnitude of the productivity 
effect depends additionally on the level of the elasticity of substitution between tasks, $\alpha$. The next proposition summarizes the first key result regarding the impact of immigration on natives relative wage structure.

Proposition 5.2. Whenever $\sigma>\epsilon$ and for a sufficiently positive finite degree of technology adoption, i.e. $0<\left\{\tilde{\varepsilon}_{L}, \tilde{\varepsilon}_{H}\right\}<\infty$, then changes in native wage structure are characterized as follows:

- Low-skill immigration induces a wage effect similar to an "unskill-bias" technological change, i.e. an relative increase in wages between low- and mediumskilled workers and between medium- and high-skilled workers;

- Medium-skill immigration generates a hump shaped relationship, i.e. mediumskill wages rise relative to both low- and high-skill wages;

- High-skill immigration has a similar wage effect compared to a "skill-bias" technological change, i.e. a monotonic increase in relative wages across the skill groups.

Proof. See the discussion in the text.

From Eqs. (5.37), (5.39), (5.40), and (5.42) is readily seen that whenever the goods demand elasticity is larger than the market power of monopolists, the direction of the efficiency effect and the labor supply effect becomes independent from the extent of the elasticity of substitution between tasks $(\alpha)$ and the initial value of the task margin. The intuition behind this result, as highlighted by Acemoglu et al. (2012), turns on two interacting forces: the market-size effect and the price effect. To illustrate these price and market-size effects, consider the low-skill immigration scenario. First, the internal reallocation of tasks between low-skilled immigrants and natives enhances the cost-efficiency of firms hiring them, raising their profits and in turn the mass of those monopolists, denoting the aforementioned direct market-size effect.

As a consequence the comparative advantages of technology mode $L$ increases, leading to an expansion of threshold margin $z_{L}$ and $z_{H}$ due to endogenous technology adoption behavior - this can be also interpreted as the indirect market size effect. This latter effect brings about a biased price effect, associated with an increase in the relative price index of goods produced with technology modes $M$ and $H$ compared to $L$. This biased price effect elicits an increase in the mass of monopolists $A_{M}$ and $A_{H}$, while counteracting the direct market size effect for technology mode $L$, i.e. a reduction in $A_{L}$ (see Eq. (5.22)). Consequently, by the standard Stolper-Samuelson theorem, the relative wage of $M$ and $H$ skilled workers rises. However, for the general equilibrium perspective, consumer preferences work in opposite direction, and thus the relative strength between these effects is ambiguous. For $\sigma>\epsilon$, the goods demand effect will be the dominating force, indicating the relative favorable shift in the demand for low-skill-intensive final goods. The empirical literature provide a range of values for these elasticities. The range of values for the elasticity of substitution across intermediates are usually estimated above 3, while between skill groups are in the range [1.5,2] (see Acemoglu et al., 2012, for further details). This implies that the empirically plausible case is whenever $\sigma<\epsilon$, which I elaborate next. 
Recalling Eqs. (5.37)-(5.42), the direction of immigration-induced changes in native wage structure depends, on the one hand, the relative strength between the productivity effect and the labor supply effect. On the other hand, it depends on the degree of the elasticity of substitution between tasks and the degree of technology adoption. Thus, it follows that for certain degree of task substitutability/complementarity the immigration-induced efficiency enhancing effect will have a beneficial effect for native workers.

Lemma 5.3 (Tasks Complementarity). Given $\sigma<\epsilon$, i.e. the goods demand elasticity is lower than the elasticity of substitution between intermediates, it follows that tasks performed by immigrants complement those performed by natives if and only if $\alpha<\min \left\{\tilde{\alpha}_{L}, \tilde{\alpha}_{H}\right\}$, where $\tilde{\alpha}_{L} \equiv \frac{(\epsilon-1) \sigma}{(\epsilon-\sigma)\left(1+\Delta_{H}\right)}, \tilde{\alpha}_{H} \equiv \frac{(\epsilon-1) \sigma}{(\epsilon-\sigma)\left(1+\Gamma_{L}\right)}$, and $\min \left\{\tilde{\alpha}_{L}, \tilde{\alpha}_{H}\right\}=$ $\max \left\{\tilde{\varepsilon}_{L} z_{L}\left(1-z_{L}\right), \tilde{\varepsilon}_{H} z_{H}\left(1-z_{H}\right)\right\}$.

Proof. See Appendix 5.A.5.

Lemma 5.3 characterizes two key properties. First, the efficiency gains accrued to natives arise up to a certain level of the elasticity of substitution across tasks. The empirical studies provide estimations that indicate the complementarity between tasks performed by immigrants and by natives. For example, Peri and Sparber (2009) estimate the value of the elasticity of substitution between manual and communicative, complex tasks in the range $[0.63,1.43]$. Now, for empirically plausible values it can be shown that the upper boundary in Lemma 5.3 lies comfortably outside of this range.

Second, the extent of task complementarity/substitutability depends also on the degree of technology adoption. Intuitively, easier adoption across various production modes will mitigate the direct market size effect of immigration, and thus leaving the wage structure independent from the elasticity $\alpha$. More precisely, as elaborated in chapter 4 , for the asymptotic case $\left\{\tilde{\varepsilon}_{L}, \tilde{\varepsilon}_{H}\right\} \rightarrow 0$, i.e. when the adoption between the different technology modes in the neighborhood of the margins, $z_{L}$ and $z_{H}$, becomes extremely easy, $\Gamma_{L}=-\Gamma_{M}=-\Delta_{H}=\Delta_{M}=1$, and $-\Delta_{L}=\Gamma_{H}=0$. This implies that the market size and the price effects will be asymptotically balanced. Utilizing these observations into Eqs. (5.37)-(5.42), the second key result can be summarized as follows

Proposition 5.3. Whenever the adoption between different technology modes becomes extremely easy, changes in native wage structure are asymptotically characterized by the immigration-induced efficiency effect due to task specialization, such that

$$
\frac{\mathrm{d} \ln \omega_{L M}^{D}}{\mathrm{~d} \ln I_{L}} \approx \tilde{\Theta}_{L}\left(I_{L}\right), \quad \frac{\mathrm{d} \ln \omega_{M H}^{D}}{\mathrm{~d} \ln I_{H}} \approx \tilde{\Theta}_{H}\left(I_{H}\right), \quad \frac{\mathrm{d} \ln \omega_{L M}^{D}}{\mathrm{~d} \ln I_{M}}=\frac{\mathrm{d} \ln \omega_{M H}^{D}}{\mathrm{~d} \ln I_{M}} \approx \tilde{\Theta}_{M}\left(I_{M}\right),
$$

while

$$
\frac{\mathrm{d} \ln \omega_{M H}^{D}}{\mathrm{~d} \ln I_{L}}=\frac{\mathrm{d} \ln \omega_{L M}^{D}}{\mathrm{~d} \ln I_{H}} \approx 0 .
$$

Proof. See the discussion in the text.

Proposition 5.3 highlights the key role of task-reallocation and technology adoption mechanisms. The latter importantly mitigates the magnitude of initial direct competition effects associated with the market size effect. However, the former indicates the novel feature of efficiency gains through task specialization (the productivity effect), supporting recent empirical evidence (cf. Ottaviano et al., 2013). The results of the analysis show that accounting exclusively for aggregate effects of endogenous technology adoption effects, as 
in several studies (cf. Beaudry et al., 2010), might provide only a partial picture of the implication of immigration-induced changes in native wage structure.

It is noteworthy that whenever the degree of technology adoption is positive and finite, i.e. $0<\left\{\tilde{\varepsilon}_{L}, \tilde{\varepsilon}_{H}\right\}<\infty$ immigration-induced changes in native wage structure depend crucially on the initial level of the task margin. This in turn determines the relative magnitude between the productivity and labor supply effects. To obtain a better idea, recall the impact of low-skill immigration on relative wage structure at the lower end of the skill distribution, Eq. (5.37). Let the first term on the right hand side be $\mathcal{H}_{L}\left(I_{L}\right) \equiv$ $\left(1-\alpha \delta\left(1-\Gamma_{L}\right)\right) \tilde{\Theta}_{L}\left(I_{L}\right)$, capturing the productivity effect, and the second term on the right hand side be $\mathcal{G}_{L}\left(I_{L}\right) \equiv \delta\left(1-\Gamma_{L}\right) \frac{I_{L}}{1-I_{L}}$, where $\delta \equiv \frac{\epsilon-\sigma}{(\epsilon-1) \sigma}$, denoting the labor supply effect. Now it can be shown that for $I_{L} \rightarrow 0, \mathcal{H}_{L}=\mathcal{G}_{L} \rightarrow 0$, while as $I_{L} \rightarrow 1, \mathcal{G}_{L} \rightarrow \infty$ and $\mathcal{H}_{L} \rightarrow\left(1-\alpha \delta\left(1-\Gamma_{L}\right)\right) \mu_{L}$, where $\mu_{L}=\frac{\mathrm{d} \ln \gamma_{L}\left(I_{L}\right)}{\mathrm{d} \ln I_{L}}$ denotes the elasticity of taskcomparative advantage schedule of immigrants. Moreover, given that $\gamma_{L}(i)$ is continuous and monotonically increasing over the unit interval, there must exist a single crossing between $\mathcal{H}_{L}$ and $\mathcal{G}_{L}$, defining a threshold margin at which both effects are balanced.

Figure 5.2: Interaction between productivity and labor supply effects

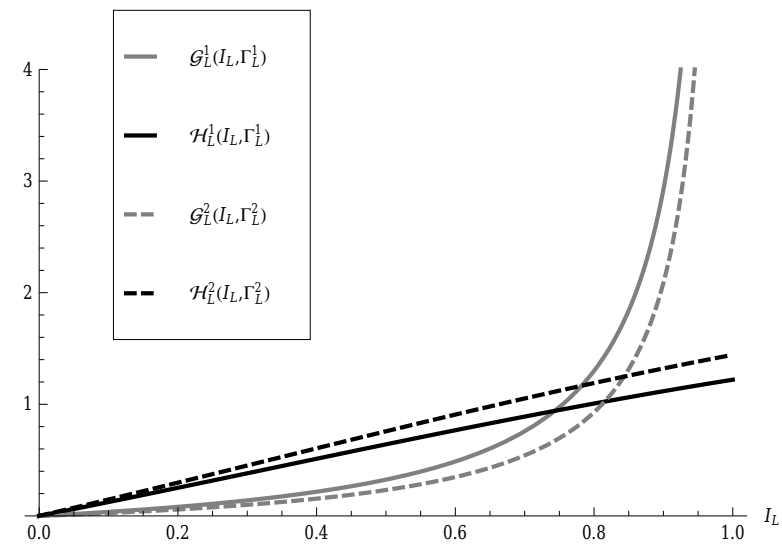

Notes: The figure shows the relationship between productivity effect and labor supply effect, for the case $\Gamma_{L}^{1}=0.4$ (solid line) and $\Gamma_{L}^{2}=0.7$ (dashed line), see text for the remaining parameter values.

Figure 5.2 depicts this relationship for the case of a log-linear functional form of $\gamma_{L}(i)=\exp \left[\mu_{L} i\right]$, for empirically plausible values of $\epsilon=3.33$ and $\sigma=1.6$ (cf. Acemoglu et al., 2012) and $\alpha=1.2$, which lies within the estimated range of values in Peri and Sparber (2009). The same relationship can be also derived from Eqs. (5.39) and (5.40) for a medium-skilled task margin, and from Eq. (5.42) for the high-skilled task margin. Moreover, the graphical illustration highlights that for higher degree of technology adoption (the dashed lines in Figure 5.2) the productivity effect becomes dominating over the entire range of task interval. Thus, the next key result can be summarized as follows.

Proposition 5.4. Given $\epsilon>\sigma$ and a sufficient degree of task complementarity, defined by Lemma 5.3, it follows that whenever the task margin is higher than a threshold $\check{I}_{k} \in(0,1)$, the immigration-induced efficiency effect is dominated by the labor supply effect, so that changes in native wage structure induced by 
- Low-skill immigration are like a "skill-bias" technological change, i.e. a relative decline in low- and medium-skilled wages compared to medium-and high-skilled wages, respectively.

- Medium-skill immigration are characterized by polarizing wage effect, i.e. a relative decline in medium-skilled wages compared to low-and high-skilled wages.

- High-skill immigration are like a "unskill-bias" technological change, i.e. a relative increase in low- and medium-skilled wages compared to medium-and highskilled wages, respectively.

Proof. See Appendix 5.A.6 and the discussion in the text.

Proposition 5.4 highlights the third key feature of the model, associated with a non-monotonic relationship between immigration and the relative magnitude between efficiency and labor supply effects. Moreover, the impact of immigration on native wage structure contrasts now those derived in Proposition 5.2. Particularly, at high initial levels of task margin, marginal increase in immigration induces a labor-saving technological effect for similarly skilled native workers, lowering their wages compared to other skill groups. However, it is worth mentioning the case when the initial level of task

Table 5.1: Immigration and native wage structure, if $\sigma<\epsilon$

\begin{tabular}{ccc|cc|cc} 
& \multicolumn{2}{c}{ Low-skill } & \multicolumn{2}{c}{ Medium-skill } & \multicolumn{2}{c}{ High-skill } \\
\hline \hline & $I_{L}<\check{I}_{L}$ & $I_{L}>\breve{I}_{L}$ & $I_{M}<\check{I}_{M}$ & $I_{M}>\breve{I}_{M}$ & $I_{H}<\check{I}_{H}$ & $I_{H}>\check{I}_{H}$ \\
\hline $\mathrm{d} \ln \omega_{L M}^{D}$ & + & - & - & + & + & + \\
$\mathrm{d} \ln \omega_{M H}^{D}$ & - & - & + & - & - & + \\
\hline
\end{tabular}

margin is lower than the threshold margin $\check{I}_{k}$, implying that the the productivity effect is dominating the labor supply effect. In this case, both low-skill and high-skill immigration scenarios induce a polarization of native wage structure, while medium-skill immigration scenario induce an inverse U-shaped wage effect. Table 5.1 summarizes these results.

Moreover, these results highlight the importance of both macro- and microeconomic structure of the model, i.e. endogenous choice production techniques at the industry level and within-firm reorganization of work, which are crucial to gain insights behind the determinants of and the interaction between various channels.

\subsection{Offshoring of intermediate inputs}

This section extends the framework with respect to the possibility of offshoring for domestic firms. As discussed above, the empirical evidence has indicated that along the value added chain jobs concentrated in the middle range of the skill distribution, characterized by high intensity of routine content of tasks, are mostly prone to offshoring. This has been put forth as the potential source of wage polarization due to offshoring (cf. Acemoglu and Autor, 2011). Thus, I assume that only intermediate goods utilized under technology mode $k=M$ are affect by offshoring. 
In doing so, I follow the exogenous offshoring approach, discussed in Acemoglu et al. (2012), where offshoring occurs in the form of relocations of intermediate inputs, similar to models of fragmentation along the value added chain (cf. Jones and Kierzkowski, 2001). More precisely, offshoring is introduced in terms of relocation of a (exogenously given) fraction of domestic jobs (intermediates), capturing changes at the extensive margin. Yet, the key features of offshoring associated with the efficiency improvement due to lower offshoring costs and the labor supply effect, reflecting the increased labor market competition between offshore and domestic workers are preserved. ${ }^{9}$ To keep the analysis tractable, the offshoring-receiving country is considered as an additional country that has become integrated in the global economy. Thus, by assumption offshoring activities by domestic firms have no impact on the mass of immigrants already existing in the home country, leaving the internal work organization (i.e. task allocation between immigrants and natives) unaffected. In the rest of this section, I describe the basic settings as well as the equilibrium conditions of offshoring, followed by the comparative static analysis.

It is assumed that domestic firms can allocate and produce an exogenously given fraction $\kappa$ of all intermediate goods $x_{M}$ over the interval $\left[0, A_{M}\right]$ abroad. However, due to implicit trade costs, cost-savings from reallocation of domestic production are limited so that $0<\kappa<\tilde{\kappa}<1$, where at the threshold $\tilde{\kappa}$, there is no arbitrage from offshoring, i.e. wages between the source and host countries of offshoring are equalized. It follows that in equilibrium a measure $\kappa A_{M}$ of monopolists produce abroad, while the remaining measure $(1-\kappa) A_{M}$ of monopolists produce at home. In what follows the variables characterizing the offshoring equilibrium are denoted by the hat-symbol "^".

Imposing labor market clearing for both medium-skilled and offshore workers and recalling the symmetry assumption, then the equilibrium output values of firms producing at home and abroad are characterized, respectively, by

$$
x_{M}=\frac{\tilde{N}_{M}}{(1-\kappa) A_{M}\left(z_{H}-z_{L}\right)}, \quad x_{O}=\frac{N_{O}}{\kappa A_{M}\left(z_{H}-z_{L}\right)},
$$

where $N_{O}$ denotes the total exogenously given endowments of offshore workers. Next, similar to the pricing-behavior of domestic monopolists, Eq. (5.10), offshoring firms charge a mark-up over the marginal cost, i.e. $p_{O}=\frac{\epsilon}{\epsilon-1} w_{O}$, where $p_{O}$ and $w_{O}$ denote the price and wage of offshoring goods and workers, respectively. The optimal demand condition for intermediate goods, Eq. (5.8), implies now the following demand conditions for offshore and domestically-produced intermediates

$$
\begin{aligned}
& p_{O}=\hat{\zeta}_{M}\left(\frac{\hat{X}_{M}}{x_{O}}\right)^{\frac{1}{\epsilon}}, \\
& p_{M}=\hat{\zeta}_{M}\left(\frac{\hat{X}_{M}}{x_{M}}\right)^{\frac{1}{\epsilon}} .
\end{aligned}
$$

\footnotetext{
${ }^{9}$ Alternatively, one could introduce offshoring following the task-based approach à la Grossman and Rossi-Hansberg (2008), which allows to account for changes at both extensive and intensive margins. See for example, Ottaviano et al. (2013) who introduce offshoring as an intermediate range of tasks between the range of tasks performed by immigrants and natives over the unit interval. However, this requires further formal structure and thus would unnecessarily complicate the analysis.
} 
Then, from Eqs. (5.44) and (5.45) and the markup-pricing behavior, it follows that the wage gap between offshore and domestic workers is given by

$$
\frac{w_{O}}{\tilde{w}_{M}}=\frac{p_{O}}{p_{M}}=\left(\frac{x_{O}}{x_{M}}\right)^{-\frac{1}{\epsilon}}=\left(\frac{N_{O}}{\tilde{N}_{M}} \frac{1-\kappa}{\kappa}\right)^{-\frac{1}{\epsilon}},
$$

where from Eq. (5.46) it is evident that $\tilde{w}_{M}>w_{O}$ for $\kappa<\tilde{\kappa} \equiv \frac{N_{O}}{N_{O}+\tilde{N}_{M}}$. Moreover, in line with recent empirical evidence, immigration of medium-skilled workers, i.e. $\frac{d \ln \tilde{N}_{M}}{\mathrm{~d} \ln I_{M}}>0$, reduces the arbitrage from offshoring, indicating that offshoring and immigration are substitutes (cf. Ottaviano et al., 2013). However, since for $I_{M} \rightarrow 1, \frac{\mathrm{d} \ln \tilde{N}_{M}}{\mathrm{~d} \ln I_{M}} \rightarrow \infty$ and thus $\tilde{\kappa} \rightarrow 0$, it is imposed that the task margin $I_{M}$ is sufficiently below unity so that positive values of offshoring exits, i.e. $0<\kappa<\tilde{\kappa}$.

Next, substituting (5.43) into the composite intermediate goods functions (5.7) for $k=M$ yields

$$
\hat{X}_{M}=A_{M}^{\frac{1}{\epsilon-1}} \frac{\hat{N}_{M}}{z_{H}-z_{L}},
$$

where

$$
\hat{N}_{M}=\left(\kappa^{\frac{1}{\epsilon}} N_{O}^{\frac{\epsilon-1}{\epsilon}}+(1-\kappa)^{\frac{1}{\epsilon}} \tilde{N}_{M}^{\frac{\epsilon-1}{\epsilon}}\right)^{\frac{\epsilon}{\epsilon-1}}
$$

denotes the weighted average, captured by the offshoring rate $\kappa$, of domestic mediumskilled and offshore workers. Eq. (5.48) reveals now an interesting feature. For given number of varieties, production is increasing in the extent of offshoring:

$$
\frac{\mathrm{d} \hat{N}_{M}}{\mathrm{~d} \kappa}=\frac{1}{\epsilon-1} \hat{N}_{M}^{1 / \epsilon}\left[\left(\frac{N_{O}}{\kappa}\right)^{\frac{\epsilon-1}{\epsilon}}-\left(\frac{\tilde{N}_{M}}{1-\kappa}\right)^{\frac{\epsilon-1}{\epsilon}}\right]>0,
$$

where $\lim _{\kappa \rightarrow 0} \mathrm{~d} \hat{N}_{M} / \mathrm{d} \kappa=\infty$ and $\lim _{\kappa \rightarrow \tilde{\kappa}} \mathrm{d} \hat{N}_{M} / \mathrm{d} \kappa=0$. This is referred to as the offshoring-induced efficiency effect, associated with an efficient reallocation of production towards countries where wages are lower (Acemoglu et al., 2012). Intuitively, at low initial values of offshoring rate, the arbitrage from offshoring is very high, due to the high offshore-domestic wage gap.

Similarly, by means of cost minimization, the equilibrium cost index of composite intermediate goods is now given by ${ }^{10}$

$$
\hat{\zeta}_{M}=A_{M}^{\frac{1}{1-\epsilon}}\left(\kappa p_{O}^{1-\epsilon}+(1-\kappa) p_{M}^{1-\epsilon}\right)^{\frac{1}{1-\epsilon}} .
$$

\footnotetext{
${ }^{10}$ More precisely, the optimization problem of a final-good producer is characterized by $\int_{0}^{\kappa A_{M}} p_{O}(j) x_{O}(j) \mathrm{d}+\int_{0}^{(1-\kappa) A_{M}} p_{M}(j) x_{M}(j) \mathrm{d}$ subject to $\hat{X}_{M}=$ $\left(\int_{0}^{\kappa A_{M}} x_{O}(j)^{\frac{\epsilon-1}{\epsilon}} \mathrm{d}+\int_{0}^{(1-\kappa) A_{M}} x_{M}(j)^{\frac{\epsilon-1}{\epsilon}} \mathrm{d}\right)^{\frac{\epsilon}{\epsilon-1}}$.
} 
Now, the zero profit condition for each final good producer $z \in\left(z_{L}, z_{H}\right)$ implies

$$
p_{M}(z) y_{M}(z)=\frac{\tilde{w}_{M} \tilde{N}_{M}+w_{O} N_{O}}{z_{H}-z_{L}}
$$

Following the same steps discussed above, the mass of monopolists is now defined by (see Appendix 5.B.6)

$$
\hat{A}_{M}=\frac{\hat{N}_{M}}{f_{M} \epsilon\left(z_{H}-z_{L}\right)}
$$

Equipped with these equilibrium conditions, the following section elaborates the comparative statics of offshoring regarding technology adoption and changes in the native wage structure.

\subsubsection{Offshoring and technology adoption}

First notice that the relative expenditure conditions defined by Eqs. (5.28) and (5.29) has to be adjusted for offshoring. Thus, utilizing Eqs. (5.47) and (5.50) in $Y_{M}$ and inserting the results in $P_{L} Y_{L} / z_{L}=P_{M} Y_{M} /\left(z_{H}-z_{L}\right)$ and $P_{M} Y_{M} /\left(z_{H}-z_{L}\right)=P_{H} Y_{H} /\left(1-z_{H}\right)$ and solving for the relative price indices yields

$$
\begin{aligned}
& \frac{P_{L}}{P_{M}}=\left(\frac{f_{L}}{f_{M}}\right)^{\frac{1}{(\epsilon-1)}}\left(\frac{\hat{N}_{M}}{\tilde{N}_{L}}\right)^{\frac{\epsilon}{(\epsilon-1)}}\left(\frac{z_{L}}{z_{H}-z_{L}}\right)^{\frac{\epsilon}{(\epsilon-1)}}, \\
& \frac{P_{M}}{P_{H}}=\left(\frac{f_{M}}{f_{H}}\right)^{\frac{1}{(\epsilon-1)}}\left(\frac{\tilde{N}_{H}}{\hat{N}_{M}}\right)^{\frac{\epsilon}{(\epsilon-1)}}\left(\frac{z_{H}-z_{L}}{1-z_{H}}\right)^{\frac{\epsilon}{(\epsilon-1)}} .
\end{aligned}
$$

Combining Eqs. (5.28') and (5.29') with Eqs. (5.3) and (5.4) yields two implicit functions adjusted for offshoring

$$
\begin{aligned}
\hat{\mathcal{F}}_{L}\left(z_{L}, z_{H}, f_{L}, f_{M}, I_{L}, I_{M}, \kappa\right) & \equiv \frac{1}{\epsilon} \ln \left(\frac{f_{L}}{f_{M}}\right)+\ln \left(\frac{\hat{N}_{M}}{\tilde{N}_{L}}\right)-\frac{(\epsilon-1)}{\epsilon} \ln \Lambda_{L}\left(z_{L}\right) \\
& +\ln \left(\frac{z_{L}}{z_{H}-z_{L}}\right)=0 \\
\hat{\mathcal{F}}_{H}\left(z_{L}, z_{H}, f_{H}, f_{M}, I_{H}, I_{M}, \kappa\right) & \equiv \frac{1}{\epsilon} \ln \left(\frac{f_{M}}{f_{H}}\right)+\ln \left(\frac{\tilde{N}_{H}}{\hat{N}_{M}}\right)-\frac{(\epsilon-1)}{\epsilon} \ln \Lambda_{H}\left(z_{H}\right) \\
& +\ln \left(\frac{z_{H}-z_{L}}{1-z_{H}}\right)=0 .
\end{aligned}
$$

from which the comparative statics for easier offshoring $(\mathrm{d} \kappa>0)$ can be conducted. Now, let $\Phi_{\kappa} \equiv \frac{\mathrm{d} \ln \hat{N}_{M}}{\mathrm{~d} \kappa}$, taking the total differentiation of the adjusted $2 \times 2$ system, Eqs. $(5.30 ')$ and $\left(5.31^{\prime}\right)$, with respect to offshoring rate $\kappa$ yields 


$$
\begin{aligned}
\left(\begin{array}{ll}
\frac{\partial \mathcal{F}_{L}}{\partial z_{L}} & \frac{\partial \mathcal{F}_{L}}{\partial z_{H}} \\
\frac{\partial \mathcal{F}_{H}}{\partial z_{L}} & \frac{\partial \mathcal{F}_{H}}{\partial z_{H}}
\end{array}\right)\left(\begin{array}{l}
\mathrm{d} \ln z_{L} \\
\mathrm{~d} \ln z_{H}
\end{array}\right) & =\left(\begin{array}{c}
-\frac{\partial \mathcal{F}_{L}}{\partial \kappa} \\
-\frac{\partial \mathcal{F}_{H}}{\partial \kappa}
\end{array}\right) \times \mathrm{d} \kappa, \\
\Leftrightarrow\left(\begin{array}{cc}
\frac{\epsilon-1}{\epsilon} \tilde{\varepsilon}_{L} z_{L}+\frac{z_{H}}{z_{H}-z_{L}} & -\frac{z_{H}}{z_{H}-z_{L}} \\
-\frac{z_{L}}{z_{H}-z_{L}} & \frac{\epsilon-1}{\epsilon} \tilde{\varepsilon}_{H} z_{H}+\frac{z_{H}\left(1-z_{L}\right)}{\left(1-z_{H}\right)\left(z_{H}-z_{L}\right)}
\end{array}\right)\left(\begin{array}{l}
\mathrm{d} \ln z_{L} \\
\mathrm{~d} \ln z_{H}
\end{array}\right) & =\left(\begin{array}{c}
-\Phi_{\kappa} \\
\Phi_{\kappa}
\end{array}\right) \times \mathrm{d} \kappa,
\end{aligned}
$$

Then, by Cramer's rule, we obtain

$$
\frac{\mathrm{d} \ln z_{L}}{\mathrm{~d} \kappa}=\frac{\mathrm{d} \ln \hat{N}_{M}}{\mathrm{~d} \kappa} \frac{\mathrm{d} \ln z_{L}}{\mathrm{~d} \ln \hat{N}_{M}}=-\Phi_{\kappa} \frac{\frac{z_{H}}{1-z_{H}}+\frac{(\epsilon-1)}{\epsilon} \tilde{\varepsilon}_{H} z_{H}}{\mathcal{D}_{J}}<0
$$

and

$$
\frac{\mathrm{d} \ln z_{H}}{\mathrm{~d} \kappa}=\frac{\mathrm{d} \ln \hat{N}_{M}}{\mathrm{~d} \kappa} \frac{\mathrm{d} \ln z_{H}}{\mathrm{~d} \ln \hat{N}_{M}}=\Phi_{\kappa} \frac{1+\frac{\epsilon-1}{\epsilon} \tilde{\varepsilon}_{L} z_{L}}{\mathcal{D}_{J}}>0
$$

Eqs. (5.53) and (5.54) reveal again the convenient structure of the model, allowing to decompose changes in the technology margins into offshoring-induced efficiency effect $\left(\Phi_{\kappa}\right)$ and changes at the extensive margins due to technology adoption behavior. The next proposition summarizes the key result.

Proposition 5.5. An exogenous increase in the offshoring rate ( $\mathrm{d} \kappa>0$ ) induces an efficiency effect for products produced under the complementing technology mode $k=M$, and thus raising the comparative advantages of adopting this technology mode. Consequently the economy becomes proportionately more specialized in technology mode $M$.

Proof. See the discussion in the text.

Thus, Propositions 5.1 and 5.5 show that both immigration of medium-skilled workers and offshoring of intermediate goods induce a cost-efficiency improvement for firms and industries employing these factors most intensively. This raises the comparative advantages of production techniques complementing these two factors. Consequently, final goods that have been produced under the technology modes $L$ and $H$ are now produced by technology mode $M$.

\subsubsection{Offshoring and native wage structure}

As discussed earlier, the relative wage structure can be derived from the equilibrium condition (5.34). Thus, following the same steps and utilizing the offshoring adjusted equilibrium conditions (5.43), (5.45), (5.47), (5.48) together with previously derived equilibrium variables $(5.16),(5.20),(5.21),(5.22),(5.24),(5.27)$, into Eq. (5.34) and 
taking ratios with respect to medium-skilled wages, yields

$$
\begin{aligned}
& \omega_{L M}^{D}=\left(\frac{z_{L}}{z_{H}-z_{L}}\right)^{\frac{\epsilon-\sigma}{(\epsilon-1) \sigma}}\left(\frac{\tilde{N}_{L}}{\hat{N}_{M}}\right)^{-\frac{\epsilon-\sigma}{(\epsilon-1) \sigma}} \hat{N}_{M}^{-\frac{1}{\epsilon}}\left(\frac{\tilde{N}_{M}}{1-\kappa}\right)^{\frac{1}{\epsilon}}\left(\frac{\Theta_{M}}{\Theta_{L}}\right) \mathcal{K}_{L M}, \\
& \omega_{M H}^{D}=\left(\frac{z_{H}-z_{L}}{1-z_{H}}\right)^{\frac{\epsilon-\sigma}{(\epsilon-1) \sigma}}\left(\frac{\hat{N}_{M}}{\tilde{N}_{H}}\right)^{-\frac{\epsilon-\sigma}{(\epsilon-1) \sigma}} \hat{N}_{M}^{\frac{1}{\epsilon}}\left(\frac{\tilde{N}_{M}}{1-\kappa}\right)^{-\frac{1}{\epsilon}}\left(\frac{\Theta_{H}}{\Theta_{M}}\right) \mathcal{K}_{M H},
\end{aligned}
$$

where $\mathcal{K}_{L M}$ and $\mathcal{K}_{M H}$ are defined as in Eqs. (5.35) and (5.36). Again Eqs. (5.55) and (5.56) allow to decompose the effect of $\kappa$ into (i) a market size effect, $\hat{N}_{M}^{-\frac{\epsilon-\sigma}{(\epsilon-1) \sigma}}$, (ii) an efficiency effect, $\hat{N}_{M}^{\frac{1}{\epsilon}}$, (iii) a technology adoption effect, $\left(\frac{z_{L}}{z_{H}-z_{L}}\right)^{\frac{\epsilon-\sigma}{(\epsilon-1) \sigma}}$ and $\left(\frac{z_{H}-z_{L}}{1-z_{H}}\right)^{\frac{\epsilon-\sigma}{(\epsilon-1) \sigma}}$, and (iv) a labor supply effect, $\left(\frac{\tilde{N}_{M}}{1-\kappa}\right)^{\frac{1}{\epsilon}}$. This latter channel is the result of the assumption that offshoring accounts only the impact at the extensive margins, and thus neglecting the beneficial effects of a reduction of offshoring costs on all intermediate inputs (intensive margins). Notice that the terms $\left(\frac{\Theta_{M}}{\Theta_{L}}\right)$ and $\left(\frac{\Theta_{H}}{\Theta_{M}}\right)$ capture the immigration-induced efficiency effects, which by contraction are insensitive to changes in offshoring $\kappa$.

I now discuss these channels in more detail. First, as mentioned above, the relative strength between the labor supply effect and the efficiency effect depend on the extent of offshoring rate. From Eq. (5.48), we get that for $\kappa \rightarrow 0, \Phi_{\kappa} \equiv \frac{\mathrm{d} \tilde{N}_{M}}{\mathrm{~d} \kappa} \rightarrow \infty$, implying that at low levels of offshoring, the efficiency effect will dominate the labor supply effect induced by offshoring. Intuitively, at low values of offshoring rate the wage gap and thus the arbitrage from offshoring will be very large for domestic firms (Acemoglu et al., 2012). However, notice that this result differs from the non-monotonic relationship discussed in Egger et al. (2013), where marginal gains from offshoring arise only at very low levels of offshoring costs, i.e. when the initial fraction of offshoring firms is high, due to the efficiency effect at the intensive margin. In this case, a decline of offshoring cost leads to favorable shift of domestic workforce from low to high productive firms.

Second, as discussed in the case of medium-skill immigration, the direct market size effect $\left(\tilde{N}_{M}\right)$ are interacting with the technology adoption effect $\left(\Delta_{M}\right.$ and $\left.\Gamma_{M}\right)$, where the relative magnitude between these two effects depends again on the extent of technology adoption in the neighborhood of the margins $z_{L}$ and $z_{H}$, captured by the elasticity parameters $\tilde{\varepsilon}_{L}$ and $\tilde{\varepsilon}_{H}$. This is the crucial departure from Acemoglu et al. (2012), where this latter effect is omitted. To see this more formally, take logs in Eqs. (5.55) and (5.56) differentiate totally with respect to $\kappa$ and make use of the comparative static results (5.53) and (5.54) to obtain

$$
\begin{aligned}
\frac{\mathrm{d} \ln \omega_{L M}^{D}}{\mathrm{~d} \kappa} & =\frac{\epsilon-\sigma}{(\epsilon-1) \sigma}\left(1+\Gamma_{M}\right) \Phi_{\kappa}-\frac{1}{\epsilon}\left(\Phi_{\kappa}-\frac{1}{1-\kappa}\right) \\
\frac{\mathrm{d} \ln \omega_{M H}^{D}}{\mathrm{~d} \kappa} & =-\frac{\epsilon-\sigma}{(\epsilon-1) \sigma}\left(1-\Delta_{M}\right) \Phi_{\kappa}+\frac{1}{\epsilon}\left(\Phi_{\kappa}-\frac{1}{1-\kappa}\right)
\end{aligned}
$$

In Eqs. (5.57) and (5.58), the first terms describe the interaction between the technology adoption effect and the direct market size effect, while the second terms denote the 
interaction between the labor supply effect and the efficiency effect. It is readily seen that the direction of the first term depends again on the relative magnitude between the final-goods demand elasticity $(\sigma)$ and the market power of monopolists $(\epsilon)$. Thus, whenever $\epsilon>\sigma$, i.e. the elasticity of substitution between intermediates is larger than the goods demand elasticity, the aforementioned price effect induced by specialization in $L$ and $H$ technology modes becomes the dominating force, and thus raising low- and high-skilled wages through the Stolper-Samuelson theorem.

However, the strength of the first terms in Eqs. (5.57) and (5.58) is mitigated by two forces. On the one hand, it depends on the degree of technology adoption in the neighborhood of the margins $z_{L}$ and $z_{H}$, denoted by $\tilde{\varepsilon}_{L}$ and $\tilde{\varepsilon}_{H}$, respectively. On the other hand, it depends on the level of offshoring rate $\kappa$. Thus, when either $\kappa \rightarrow \tilde{\kappa}$ (recall that $\lim _{\kappa \rightarrow \tilde{\kappa}} \Phi_{\kappa}=0$ ), or $\left\{\tilde{\varepsilon}_{L}, \tilde{\varepsilon}_{H}\right\} \rightarrow 0$ (recall that $\lim _{\left\{\tilde{\varepsilon}_{L}, \tilde{\varepsilon}_{H}\right\} \rightarrow 0} \Delta_{M}=-\Gamma_{M}=1$ ), the first terms vanish. Therefore, for $\kappa<\tilde{\kappa}$ and a sufficient positive and finite degree of technology adoption, it follows from Eqs. (5.57) and (5.58) that the offshoring-induced efficiency effect will be the dominating force for a certain degree of technology adoption. The next proposition summarizes this result.

Proposition 5.6. Given a positive and finite degree of technology adoption, i.e. $\left\{\tilde{\varepsilon}_{L}, \tilde{\varepsilon}_{H}\right\}>0$, an exogenous increase in offshoring rate $\kappa$ induces an efficiency effect which is counteracting the technology adoption effect whenever $\epsilon>\sigma$. There exists a threshold degree of technology adoption $\chi \equiv \frac{\epsilon^{2}+\sigma-2 \sigma \epsilon}{\epsilon(\epsilon-\sigma)}$, which determines a lower boundary for changes in the relative range of final goods. Whenever $\min \left\{\left|\Gamma_{M}\right|, \Delta_{M}\right\}>\chi$, the efficiency effect will be the dominating force. Moreover, for initial low values of offshoring rate the efficiency effect will also dominate the labor supply effect.

Proof. See Appendix 5.A.7.

The results in Proposition 5.6 highlight again the importance of technology adoption behavior and the rich pattern of interaction between various forces. Intuitively, the easier the technology adoption is in the neighborhood of the margins $z_{L}$ and $z_{H}$, the stronger will be changes in the relative range of final goods, i.e. high values of $\left|\Gamma_{M}\right|$ and $\Delta_{M}$. Thus, whenever the market power of monopolists is larger than the final-goods demand elasticity $(\epsilon>\sigma)$, technology adoption effect will mitigate the extent of the market size effect. Now equipped with these results, the next proposition summarizes the main results regarding the impact of offshoring on natives relative wage structure.

Proposition 5.7. Given the results in Proposition 5.6, changes in natives relative wage structure due to an exogenous increase in offshoring rate $(\mathrm{d} \kappa>0)$ are characterized by a

(i) Medium-skill-augmenting wage effect, associated with a relative increase of medium-skilled wages compared to both low- and high-skilled wages - a hump shaped relationship, whenever the offshoring rate is initially low.

(ii) Polarizing wage effect, associated with a relative decline of medium-skilled wages compared to both low- and high-skilled wages - a U-shaped relationship, whenever the offshoring rate is initially high.

Proof. See the discussion in the text.

Comparing the results in Proposition 5.7 with those of the case of medium-skill immigration discussed in Proposition 5.2 and Proposition 5.4 it is readily seen that 
both types of labor shock have a similar impact on natives relative wage structure, but the adjustment mechanism is different. Medium-skill immigration leads to an internal reorganization of work (i.e. task reallocation), where natives react endogenously by specializing in communicative tasks, while offshoring of medium skill-intensive jobs (intermediates) affects both immigrants and natives proportionately, leaving task intensity unaffected. Although the latter is by construction, the results highlight that the direction of distributional impact of both medium-skill immigration and offshoring for native workers depends crucially on their initial share in the economy.

It is also worth noticing that the results in Propositions 5.2, 5.4, and 5.7 differ substantially from those derived in Olney (2012) and Ottaviano et al. (2013), who investigate jointly the impact of offshoring and immigration. The key arguments are that implicit assumption of perfect substitutability between immigrants and natives, e.g. as in Olney (2012), miss to capture important aspects of potential efficiency improvements highlighted by the recent empirical evidence, and that accounting only for partial equilibrium effects, e.g. as in Ottaviano et al. (2013), neglect important general equilibrium implications.

\subsection{Conclusion}

Immigration and offshoring of domestic jobs are two phenomena that have considerably shaped the public debate regarding their potential labor market effects in many advanced countries. Thus, understanding the determinants of the impact of offshoring and immigration is one of the key objectives of both policy makers and researchers. This paper has analyzed jointly the impact of these two phenomena of globalized labor services on native wage structure and how their effects are interlinked. In doing so, I develop an integrated theoretical framework that features a richer structure of the economy consisting of firm heterogeneity, endogenous technology adoption, monopolistic competition, and skill heterogeneity. Moreover, the systematic framework allows to account for various potential adjustment channels of the receiving economy to offshoring and immigration shocks, and to investigate the determinants of and the interaction between these different forces from a general equilibrium perspective. An important feature allows capture the relationship between specialization in technology modes and increasing returns to scale.

The theoretical analysis provides several novel insights. First, immigration and offshoring induce three main channels: (i) a market size effect, (ii) a technology adoption effect, and (iii) an efficiency effect. More importantly, the relative magnitude between the first two effects is crucially determined by the degree of technology adoption. In addition, the direction of each of these two effects is importantly determined by the relative magnitude between consumer preferences (i.e. final-goods demand elasticity) and the market power of monopolists (i.e. the elasticity of substitution between intermediate goods). An interesting result of the analysis reveals that whenever the degree of technology adoption between different production modes becomes very easy, the market size and technology adoption effects are asymptotically balanced. Thus, the remaining channel is the productivity effect, associated with the cost-efficiency enhancing. Third, the magnitude of the productivity effect is stronger at low initial levels of task shares assigned 
to immigrants and of offshoring rate. The intuition is that at higher levels the labor market competition among domestic workers, associated with the direct displacement effect due to task reallocation in the case of immigration, or job relocation in the case of offshoring, becomes dominating. These results highlight the rich pattern of interaction between different forces. Nevertheless, the tractability of the framework permits to derive clear conditions regarding when some forces dominate others, and thus enabling to make clear predictions regarding the impacts on native wage structure.

The comparative statics reveal the following predictions regarding immigration-induced changes in the relative wage structure of native workers. Whenever consumer preferences react less elastic relative to the market power of monopolists, low- and high-skilled immigration induce wage effects similar to "unskill-" and "skilled-biased" technological changes, respectively. Medium-skilled immigration generates both a hump-shaped relationship. The rationale behind these results is twofold. On the one hand, profitmaximizing incentives elicits an endogenous increase in the mass of monopolists hit by immigration shock - indicating the direct market size effect. On the other hand, immigration raises the comparative advantages of the complementing technology mode by lowering its cost index. Due to the endogenous choice for technology modes, the range of final goods produced under the complementing technology mode is raised - indicating the technology adoption effect. However, this latter effect induces, in addition, a price effect favoring final goods produced under the competing technology modes as the economy becomes more specialized in those production techniques. By the standard StolperSamuelson theorem, this price effect is translated into wage effects of the respective skill group. However, with strong elastic consumer preferences, the demand shifts in favor of cheaper final goods and labor that were hit by the immigration shock, and thus overcompensating the initial adverse effects.

More interestingly, in the empirically plausible case, i.e. when the market power of monopolists is larger than the goods demand elasticity, the predictions of distributional effects of immigration depends crucially on the level of task shares assigned to immigrants. Thus, at low levels of task shares, low- and high-skilled immigration both generate a polarizing wage effect, while medium-skilled immigration induces again a hump-shaped wage effect. This is due to strong productivity effects at low initial task shares, and the spill-over effects through the scale and price externalities mentioned above. In the case of offshoring, changes in the wage structure of native workers is similar to those induced by medium-skilled immigration. However, an interesting result is that even considering the long-run scenario, i.e. when market size and technology adoption are balanced, the remaining channel is the productivity effect induced by gains due to efficient reallocation of tasks and jobs. 


\section{Appendix}

\section{A Proofs}

\section{A.1 Proof of Lemma 5.1}

The proof of the optimal task assignment between immigrants and natives can be conducted in two steps. First, I derive the optimal amount of composite factor labor $L_{k}$ for a given $I_{k}$, and then derive the optimal task margin $I_{k}$. If $L_{k}^{D}$ and $L_{k}^{F}$ are the amounts of native and immigrant workers employed by any intermediate firm $j \in\left[0, A_{k}\right]$, it must satisfy

$$
\begin{aligned}
& \int_{0}^{I_{k}} l_{k}^{F}(i) \mathrm{d} i=L_{k}^{F}, \\
& \int_{I_{k}}^{1} l_{k}^{D}(i) \mathrm{d} i=L_{k}^{D} .
\end{aligned}
$$

The optimal amount of workers across the respective range of tasks is obtained by minimizing the cost

$$
\min _{l_{k}^{F}(i), l_{k}^{D}(i)} \int_{0}^{I_{k}} w_{k}^{F} l_{k}^{F}(i) \mathrm{d} i+\int_{I_{k}}^{1} w_{k}^{D} l_{k}^{D}(i) \mathrm{d} i \quad \text { s.t. }
$$

The first-order conditions yield

$$
\begin{aligned}
& w_{k}^{D}=\tilde{w}_{k}\left(\frac{\tilde{L}_{k}}{l_{k}^{D}(i)}\right)^{\frac{1}{\alpha}} \\
& w_{k}^{F}=\tilde{w}_{k}\left(\frac{\tilde{L}_{k}}{l_{k}^{F}(i)}\right)^{\frac{1}{\alpha}}\left(\gamma_{k}(i)\right)^{\frac{1-\alpha}{\alpha}},
\end{aligned}
$$

where $\tilde{w}_{k}$ denotes the Lagrangian multiplier. It follows from the optimality conditions (5.A.62) and (5.A.63)

$$
\begin{array}{ll}
l_{k}^{D}(i)=l_{k}^{D}\left(i^{\prime}\right), & \text { for } \quad i, i^{\prime} \in\left(I_{k}, 1\right] \\
l_{k}^{F}(i)=l_{k}^{F}\left(i^{\prime}\right)\left(\frac{\gamma_{k}(i)}{\gamma_{k}\left(i^{\prime}\right)}\right)^{1-\alpha}, & \text { for } \quad i, i^{\prime} \in\left[0, I_{k}\right]
\end{array}
$$

Thus, for the domestic labor it follows from equations (5.A.60) and (5.A.64) that

$$
l_{k}^{D}(i)=\frac{L_{k}^{D}}{1-I_{k}}
$$


For the allocation of offshore workers, note that from Eq. (5.A.65), it must hold

$$
l_{k}^{F}(i)=l_{k}^{F}\left(I_{k}\right)\left(\frac{\gamma_{k}(i)}{\gamma_{k}\left(I_{k}\right)}\right)^{1-\alpha}, \quad \text { for all } i \in\left[0, I_{k}\right]
$$

Plugging Eq. (5.A.67) into Eq. (5.A.59), one can solve for $l_{k}^{F}\left(I_{k}\right)$ and substituting this expression back into Eq. (5.A.67) yields

$$
l_{k}^{F}(i)=\left(\frac{\gamma_{k}(i)^{1-\alpha}}{\int_{0}^{I_{k}} \gamma_{k}(i)^{1-\alpha} \mathrm{d} i}\right) L_{k}^{F}
$$

Thus, substituting Eqs. (5.A.66) and (5.A.68) into Eq. (5.11) and recalling that labor is the sole of production in the intermediate sector, i.e. $x_{k}=\tilde{L}$, yields

$$
x_{k}=\tilde{L}_{k}=\left(\left[\int_{0}^{I_{k}} \gamma_{k}(i)^{1-\alpha} \mathrm{d} i\right]^{\frac{1}{\alpha}}\left(L_{k}^{F}\right)^{\frac{\alpha-1}{\alpha}}+\left[1-I_{k}\right]^{\frac{1}{\alpha}}\left(L_{k}^{D}\right)^{\frac{\alpha-1}{\alpha}}\right)^{\frac{\alpha}{\alpha-1}}
$$

Now to prove that the optimal fraction of offshoring $I_{k}$ is cost efficient, substitute the first-order conditions (5.A.62) and (5.A.63) for $l_{k}^{D}(i)$ and $l_{k}^{F}(i)$, respectively, into Eq. (5.11) and manipulate to obtain

$$
\tilde{w}_{k}=\left(\left(\int_{0}^{I_{k}} \gamma_{k}(i)^{1-\alpha} \mathrm{d} i\right)\left(w_{k}^{F}\right)^{1-\alpha}+\left[1-I_{k}\right]\left(w_{k}^{D}\right)^{1-\alpha}\right)^{\frac{1}{1-\alpha}}
$$

Thus, Eq. (5.A.70) denotes the marginal cost of any differentiated-good producer $j \in$ $\left[0, A_{k}\right]$ employing immigrant and native workers. Now, the optimal choice of marginal task $I_{k}$ is obtained by minimizing $\tilde{w}_{k}$ with respect to $I_{k}$ :

$$
\frac{\mathrm{d} \tilde{w}_{k}}{\mathrm{~d} I_{k}}=\frac{1}{1-\alpha} \tilde{w}_{k}^{\alpha}\left(\gamma_{k}\left(I_{k}\right)^{1-\alpha}\left(w_{k}^{F}\right)^{1-\alpha}-\left(w_{k}^{D}\right)^{1-\alpha}\right)=0
$$

Thus, we get that $\frac{\mathrm{d} \tilde{w}_{k}}{\mathrm{~d} I_{k}}=0$ if and only if

$$
\gamma_{k}\left(I_{k}\right) w_{k}^{F}=w_{k}^{D}
$$

so that an intermediate-good producer allocates tasks to immigrants if and only if $\gamma_{k}(i) w_{k}^{F} \leq w_{k}^{D}$

\section{A.2 Derivation of equilibrium labor composite and marginal costs}

From (5.A.62) and (5.A.63) the inverse relative labor demand between offshore and medium-skilled workers is defined by 


$$
\frac{w_{k}^{F}}{w_{k}^{D}}=\left(\frac{l_{k}^{F}(i)}{l_{k}^{D}(i)}\right)^{-\frac{1}{\alpha}}\left[\gamma_{k}(i)\right]^{\frac{1-\alpha}{\alpha}},
$$

and using Eqs. (5.A.66) and (5.A.68) yields

$$
\frac{w_{k}^{F}}{w_{k}^{D}}=\left(\frac{L_{k}^{F}}{L_{k}^{D}}\right)^{-\frac{1}{\alpha}} \frac{\left[\int_{0}^{I_{k}} \gamma_{k}(i)^{1-\alpha} \mathrm{d} i\right]^{\frac{1}{\alpha}}}{\left(1-I_{k}\right)^{1 / \alpha}}
$$

Finally, utilizing the overall labor market clearing condition derived in Eqs. (5.17), (5.18), and (5.19) the previous equation can be written as

$$
\frac{w_{k}^{F}}{w_{k}^{D}}=\left(\frac{N_{k}^{F}}{N_{k}^{D}}\right)^{-\frac{1}{\alpha}}\left(\frac{\vartheta_{k}\left(I_{k}\right)}{(1-I)}\right)^{1 / \alpha},
$$

where $\vartheta_{k}\left(I_{k}\right) \equiv\left(\int_{0}^{I} \gamma_{k}(i)^{1-\alpha} \mathrm{d} i\right)$. Then, combining (5.A.71) with the cost-efficient condition task allocation, Eq. (5.13), yields

$$
\frac{1}{\gamma_{k}\left(I_{k}\right)}=\left(\frac{N_{k}^{F}}{N_{k}^{D}}\right)^{-\frac{1}{\alpha}}\left(\frac{\vartheta_{k}\left(I_{k}\right)}{(1-I)}\right)^{1 / \alpha}
$$

Next, solving the previous derived equation for $N_{k}^{F}$ and combining the result with the labor market clearing conditions, Eqs. (5.17)-(5.19), to insert into Eq. (5.A.69), yields

$$
\begin{aligned}
x_{k} & =\frac{\tilde{N}_{k}}{\mathcal{S}_{k} A_{k}} \\
\tilde{N}_{k} & =\left(\left[\int_{0}^{I_{k}} \gamma_{k}(i)^{1-\alpha} \mathrm{d} i\right]^{\frac{1}{\alpha}}\left(N_{k}^{F}\right)^{\frac{\alpha-1}{\alpha}}+\left(1-I_{k}\right)^{\frac{1}{\alpha}}\left(N_{k}^{D}\right)^{\frac{\alpha-1}{\alpha}}\right)^{\frac{\alpha}{\alpha-1}} \\
& =\left(\vartheta_{k}\left(I_{k}\right)^{\frac{1}{\alpha}}\left(N_{k}^{F}\right)^{\frac{\alpha-1}{\alpha}}+\left(1-I_{k}\right)^{\frac{1}{\alpha}}\left(N_{k}^{D}\right)^{\frac{\alpha-1}{\alpha}}\right)^{\frac{\alpha}{\alpha-1}} .
\end{aligned}
$$

Now, using Eq. (5.A.72) into the previous equation and manipulating slightly yields

$$
\tilde{N}_{k}=\left(\vartheta_{k}\left(I_{k}\right) \gamma_{k}\left(I_{k}\right)^{\alpha-1}+\left(1-I_{k}\right)\right)^{\frac{\alpha}{\alpha-1}} \frac{N_{k}^{D}}{1-I_{k}}=\mathcal{M}_{k}\left(\Theta_{k}, I_{k}\right) N_{k}^{D},
$$

and from Eqs. (5.13) and (5.A.70) we obtain

$$
\tilde{w}_{k}=\left(\vartheta_{k}\left(I_{k}\right) \gamma_{k}\left(I_{k}\right)^{\alpha-1}+1-I_{k}\right)^{\frac{1}{1-\alpha}} w_{k}^{D}=\Theta_{k}\left(I_{k}\right) w_{k}^{D},
$$

where $\Theta_{k}\left(I_{k}\right)$ denotes the generalized measure of marginal cost of task production. 


\section{A.3 Proof of Lemma 5.2}

Notice, first, that Eq. (5.A.72) implies that task margin $I_{k}$ depends only on the endowments of immigrant and native workers. Rearranging Eq. (5.A.72) and taking $\operatorname{logs}$, yields $\ln \left(\frac{N_{k}^{F}}{N_{k}^{D}}\right)=\alpha \ln \gamma_{k}\left(I_{k}\right)+\ln \vartheta_{k}\left(I_{k}\right)-\ln \left(1-I_{k}\right)$ Now, recalling the property of $\gamma_{k}^{\prime}(i)>0$ and differentiating this expression with respect to $N_{k}^{F}$ we get

$$
\frac{\mathrm{d} \ln I_{k}}{\mathrm{~d} \ln N_{k}^{F}}=\left(\alpha \mu_{\gamma_{k}}+\frac{\gamma_{k}\left(I_{k}\right)^{1-\alpha} I_{k}}{\vartheta_{k}\left(I_{k}\right)}+\frac{I_{k}}{1-I_{k}}\right)^{-1}>0 .
$$

From Eq. (5.A.76), we can compute the impact of changes in the task margin on the marginal cost of monopolists. Differentiating (5.A.76) w.r.t. $I_{k}$ yields

$$
\frac{\mathrm{d} \ln \Theta_{k}\left(I_{k}\right)}{\mathrm{d} \ln I_{k}}=-\Theta_{k}\left(I_{k}\right)^{\alpha-1} \vartheta_{k}\left(I_{k}\right) \gamma_{k}\left(I_{k}\right)^{\alpha-1} \mu_{\gamma_{k}}<0,
$$

where $\mu_{\gamma_{k}} \equiv \frac{\gamma_{k}^{\prime}\left(I_{k}\right) I_{k}}{\gamma_{k}\left(I_{k}\right)}>0$ denotes the elasticity of comparative advantage schedule of immigrants with respect to task margin $I_{k}$. Moreover, utilizing the outcome in Eq. (5.A.78), it follows from Eqs. (5.A.75) and (5.A.76) that, for $I_{k}>0$, the labor composite is increasing and the marginal cost of intermediate-good firm is decreasing in task margin $I_{k}$, i.e.

$$
\begin{aligned}
& \frac{\mathrm{d} \ln \tilde{N}_{k}}{\mathrm{~d} \ln I_{k}}=-\frac{\mathrm{d} \ln \mathcal{M}_{k}}{\mathrm{~d} \ln I_{k}}=-\alpha \frac{\mathrm{d} \ln \Theta_{k}\left(I_{k}\right)}{\mathrm{d} \ln I_{k}}+\frac{I_{k}}{1-I_{k}}>0 \\
& \frac{\mathrm{d} \ln \tilde{w}_{k}}{\mathrm{~d} \ln I_{k}}=\frac{\mathrm{d} \ln \Theta_{k}\left(I_{k}\right)}{\mathrm{d} \ln I_{k}}<0
\end{aligned}
$$

\section{A.4 Proof of Proposition 5.1}

The computation of the comparative statics for the other exogenous changes is similar to the one conducted for changes in the task margin $I_{M}$, associated with medium-skill immigration, recall Eq. (5.A.77). Notice that by Eq. (5.A.72) task margin $I_{k}$ depends only on the endowments of skill type $k$. Recall the definition $\Psi_{k}=-\frac{\mathrm{d} \ln \mathcal{M}_{k}\left(I_{k}\right)}{\mathrm{d} \ln I_{k}}>0$ and differentiate the implicit $2 \times 2$ system, Eqs. (5.30) and (5.31) with respect to changes in the task margins and the fixed costs, yields:

- Low-skill immigration and $L$-fixed costs:

$$
\begin{aligned}
\frac{\mathrm{d} \ln z_{L}}{\mathrm{~d} \ln I_{L}} & =\frac{\mathrm{d} \ln z_{L}}{\mathrm{~d} \ln \mathcal{M}_{L}} \frac{\mathrm{d} \ln \mathcal{M}_{L}}{\mathrm{~d} \ln I_{L}}=\Psi_{L} \frac{\frac{\epsilon-1}{\epsilon} \tilde{\varepsilon}_{H} z_{H}+\frac{z_{H}\left(1-z_{L}\right)}{\left(1-z_{H}\right)\left(z_{H}-z_{L}\right)}}{\mathcal{D}_{J}}>0 \\
\frac{\mathrm{d} \ln z_{L}}{\mathrm{~d} \ln f_{L}} & =-\frac{1}{\Psi_{L}} \frac{\mathrm{d} \ln z_{L}}{\mathrm{~d} \ln I_{L}}<0
\end{aligned}
$$


and

$$
\begin{aligned}
\frac{\mathrm{d} \ln z_{H}}{\mathrm{~d} \ln I_{L}} & =\frac{\mathrm{d} \ln z_{H}}{\mathrm{~d} \ln \mathcal{M}_{L}} \frac{\mathrm{d} \ln \mathcal{M}_{L}}{\mathrm{~d} \ln I_{L}}=\Psi_{L} \frac{\frac{z_{L}}{\left(z_{H}-z_{L}\right)}}{\mathcal{D}_{J}}>0 . \\
\frac{\mathrm{d} \ln z_{H}}{\mathrm{~d} \ln f_{L}} & =-\frac{1}{\Psi_{L}} \frac{\mathrm{d} \ln z_{H}}{\mathrm{~d} \ln I_{L}}<0
\end{aligned}
$$

- High-skill immigration and $H$-fixed costs:

$$
\begin{aligned}
\frac{\mathrm{d} \ln z_{L}}{\mathrm{~d} \ln I_{H}} & =\frac{\mathrm{d} \ln z_{L}}{\mathrm{~d} \ln \mathcal{M}_{H}} \frac{\mathrm{d} \ln \mathcal{M}_{H}}{\mathrm{~d} \ln I_{H}}=-\Psi_{H} \frac{\frac{z_{H}}{\left(z_{H}-z_{L}\right)}}{\mathcal{D}_{J}}<0, \\
\frac{\mathrm{d} \ln z_{L}}{\mathrm{~d} \ln f_{H}} & =-\frac{1}{\Psi_{H}} \frac{\mathrm{d} \ln z_{L}}{\mathrm{~d} \ln I_{H}}>0
\end{aligned}
$$

and

$$
\begin{aligned}
& \frac{\mathrm{d} \ln z_{H}}{\mathrm{~d} \ln I_{H}}=\frac{\mathrm{d} \ln z_{H}}{\mathrm{~d} \ln \mathcal{M}_{H}} \frac{\mathrm{d} \ln \mathcal{M}_{H}}{\mathrm{~d} \ln I_{H}}=-\Psi_{H} \frac{\frac{\epsilon-1}{\epsilon} \tilde{\varepsilon}_{L} z_{L}+\frac{z_{H}}{\left(z_{H}-z_{L}\right)}}{\mathcal{D}_{J}}<0 . \\
& \frac{\mathrm{d} \ln z_{H}}{\mathrm{~d} \ln f_{H}}=-\frac{1}{\Psi_{H}} \frac{\mathrm{d} \ln z_{H}}{\mathrm{~d} \ln I_{H}}>0
\end{aligned}
$$

where the Jacobi is given by

$$
\mathcal{D}_{J}=\left(\frac{\epsilon-1}{\epsilon} \tilde{\varepsilon}_{L} z_{L}+\frac{1}{z_{L}}\right)\left(\frac{\epsilon-1}{\epsilon} \tilde{\varepsilon}_{H} z_{H}+\frac{1-z_{L}}{\left(1-z_{H}\right)\left(z_{H}-z_{L}\right)}\right)+\frac{1}{z_{H}-z_{L}}\left(\frac{\epsilon-1}{\epsilon} \tilde{\varepsilon}_{H} z_{H}+\frac{1}{1-z_{H}}\right)>\text { (45.A.89) }
$$

Moreover, from (5.A.81) and (5.A.83) it can be readily verified that

$$
\frac{\mathrm{d} \ln z_{H}-\mathrm{d} \ln z_{L}}{\mathrm{~d} \ln I_{L}}=-\frac{\Psi_{L}}{\mathcal{D}_{J}}\left(\frac{\epsilon-1}{\epsilon} \tilde{\varepsilon}_{H} z_{H}+\frac{1}{\left(1-z_{H}\right)}\right)<0
$$

and similarly from (5.A.85) and (5.A.87)

$$
\frac{\mathrm{d} \ln z_{H}-\mathrm{d} \ln z_{L}}{\mathrm{~d} \ln I_{H}}=-\frac{\Psi_{H}}{\mathcal{D}_{J}}\left(\frac{\epsilon-1}{\epsilon} \tilde{\varepsilon}_{L} z_{L}\right)<0
$$

Since $\Psi_{k}\left(I_{k}\right)$ depends on the initial level of the task margin, it follows from Eq. (5.A.79) that for any $k=\{L, M, H\}$

$$
\begin{gathered}
\lim _{I_{k} \rightarrow 0} \Psi_{k}=0 \Rightarrow \lim _{I_{k} \rightarrow 0}\left|\frac{\mathrm{d} \ln z_{L}}{\mathrm{~d} \ln I_{k}}\right|=\left|\frac{\mathrm{d} \ln z_{H}}{\mathrm{~d} \ln I_{k}}\right|=0 \\
\lim _{I_{k} \rightarrow 1} \Psi_{k}=\infty \Rightarrow \lim _{I_{k} \rightarrow 1}\left|\frac{\mathrm{d} \ln z_{L}}{\mathrm{~d} \ln I_{k}}\right|=\left|\frac{\mathrm{d} \ln z_{H}}{\mathrm{~d} \ln I_{k}}\right|=\infty
\end{gathered}
$$




\section{A.5 Proof of Lemma 5.3}

Recall the thresholds of the elasticity of substitution between tasks,

$$
\begin{aligned}
\tilde{\alpha}_{L} & \equiv \frac{(\epsilon-1) \sigma}{(\epsilon-\sigma)\left(1-\Gamma_{L}\right)}, \\
\tilde{\alpha}_{H} & \equiv \frac{(\epsilon-1) \sigma}{(\epsilon-\sigma)\left(1+\Delta_{H}\right)},
\end{aligned}
$$

Thus, for the relative strength between the two thresholds we obtain

$$
\begin{aligned}
& \tilde{\alpha}_{L} \lesseqgtr \tilde{\alpha}_{H} \\
& \Gamma_{L} \lesseqgtr-\Delta_{H}
\end{aligned}
$$

Now substituting the definitions of $\Gamma_{L}$ and $\Delta_{H}$ from Eqs. (5.B.107) and (5.B.112), respectively, and manipulating yields

$$
\begin{aligned}
\frac{1}{\mathcal{D}_{J}}\left(\frac{\epsilon-1}{\epsilon} \frac{\tilde{\varepsilon}_{H} z_{H} z_{H}}{\left(z_{H}-z_{L}\right)}+\frac{z_{H}}{\left(1-z_{H}\right)\left(z_{H}-z_{L}\right)}\right) & \lesseqgtr \frac{1}{\mathcal{D}_{J}}\left(\frac{\epsilon-1}{\epsilon} \frac{\tilde{\varepsilon}_{L} z_{L}\left(1-z_{L}\right)}{\left(1-z_{H}\right)\left(z_{H}-z_{L}\right)}+\frac{z_{H}}{\left(z_{H}-z_{L}\right)\left(1-z_{H}\right)}\right) \\
\tilde{\varepsilon}_{H} z_{H}\left(1-z_{H}\right) & \lesseqgtr \tilde{\varepsilon}_{L} z_{L}\left(1-z_{L}\right)
\end{aligned}
$$

\section{A.6 Proof of Proposition 5.4}

The proof of the existent of threshold margins $\check{I}_{L}$ and $\breve{I}_{H}$ can be straightforwardly verified as follows. Given $0<\left\{\tilde{\varepsilon}_{L}, \tilde{\varepsilon}_{H}\right\}<\infty$ and the functional properties of $\gamma_{k}(i)$, it follows from Eqs. (5.37) and (5.42), respectively,

$$
\begin{aligned}
\frac{\mathrm{d} \ln \omega_{L M}}{\mathrm{~d} \ln I_{L}} & =\mathcal{H}_{L}\left(\check{I}_{L}\right)-\mathcal{G}_{L}\left(\check{I}_{L}\right)=0, \\
\frac{\mathrm{d} \ln \omega_{M H}}{\mathrm{~d} \ln I_{H}} & =\mathcal{H}_{H}\left(\check{I}_{H}\right)-\mathcal{G}_{H}\left(\check{I}_{H}\right)=0,
\end{aligned}
$$

so that

$$
\mathcal{H}_{k}\left(I_{k}\right) \lesseqgtr \mathcal{G}_{k}\left(I_{k}\right), \quad \text { for } \quad I_{k} \gtreqless \check{I}_{k}, k=\{L, H\} .
$$

The proof of the existent of threshold margin $\breve{I}_{M}$ needs further elaboration. From Eqs. (5.39) and (5.40) two threshold task margins are obtained at which $\mathrm{d} \ln \omega_{L M}^{D}=0$ and $\mathrm{d} \ln \omega_{M H}^{D}=0$. Define these two thresholds, respectively, by the following implicit functions

$$
\begin{aligned}
& \mathcal{I}_{1, M} \equiv\left(1-\alpha \delta\left(1+\Gamma_{M}\right)\right) \tilde{\theta}_{M}\left(\check{I}_{1, M}\right)-\delta\left(1+\Gamma_{M}\right) \frac{\check{I}_{1, M}}{1-\check{I}_{1, M}}=0 \\
& \mathcal{I}_{2, M} \equiv\left(1-\alpha \delta\left(1-\Delta_{M}\right)\right) \tilde{\theta}_{M}\left(\check{I}_{2, M}\right)-\delta\left(1-\Delta_{M}\right) \frac{\check{I}_{2, M}}{1-\check{I}_{2, M}}=0
\end{aligned}
$$


Thus, it is readily seen from Eqs. (5.A.96) and (5.A.97) that if $-\Gamma_{M}=\Delta_{M}$, we get that $\check{I}_{1, M}=\check{I}_{2, M}=\breve{I}_{M}$. Moreover, using the definitions of $\Gamma_{M}$ and $\Delta_{M}$ from Eqs. (5.B.109) and (5.B.110), respectively, we get that

$$
\begin{array}{r}
\frac{1}{\mathcal{D}_{J}}\left(\frac{z_{H}}{\left(z_{H}-z_{L}\right)\left(1-z_{H}\right)}+\frac{\epsilon-1}{\epsilon}\left(\tilde{\varepsilon}_{L}+\tilde{\varepsilon}_{H}\right) \frac{z_{H}}{\left(z_{H}-z_{L}\right)}\right) \\
\frac{1}{\mathcal{D}_{J}}\left(\frac{z_{H}}{\left(1-z_{H}\right)\left(z_{H}-z_{L}\right)}+\frac{\epsilon-1}{\epsilon}\left(\frac{z_{H}\left(1-z_{L}\right)}{\left(1-z_{H}\right)\left(z_{H}-z_{L}\right)} \tilde{\varepsilon}_{L}+\frac{z_{L}}{z_{H}-z_{L}} \tilde{\varepsilon}_{H}\right)\right)
\end{array}
$$

which after some manipulation yields

$$
\tilde{\varepsilon}_{H}\left(1-z_{H}\right) \lesseqgtr \tilde{\varepsilon}_{L} z_{L}
$$

This in turn implies that

$$
\check{I}_{1, M} \lesseqgtr \check{I}_{2, M}
$$

To verify (5.A.99), it is sufficient to show that either from (5.A.96), $\frac{\partial \mathcal{I}_{1, M}}{\partial \Gamma_{M}}<0$, or from (5.A.97), $\frac{\partial \mathcal{I}_{2, M}}{\partial \Delta_{M}}>0$. Thus, differentiating Eq. (5.A.97) with respect to $\Delta_{M}$, we obtain

$$
\frac{\mathrm{d} \check{I}_{2, M}}{\mathrm{~d} \Delta_{M}}=\frac{\alpha \delta \tilde{\Theta}_{M}\left(\check{I}_{2, M}\right)+\delta \check{I}_{2, M} /\left(1-\check{I}_{2, M}\right)}{\delta\left(1-\check{\Gamma}_{M}\right) \frac{1}{\left(1-\check{I}_{1, M}\right)^{2}}-\left(1-\alpha \delta\left(1-\check{\Gamma}_{M}\right) \frac{\partial \tilde{\Theta}_{M}\left(\check{I}_{2, M}\right)}{\partial \check{I}_{2, M}}\right.}>0
$$

where by the functional properties discussed above the denominator is always positive. Thus, for an asymmetric degree of technology adoption in the neighborhood of the margins $z_{L}$ and $z_{H}$, there exist two task margins, implying that the productivity (labor supply) effect dominates unambiguously the labor supply (productivity) effect when ever the task margin is lower (higher) than the minimum (maximum) of the two thresholds, i.e. $I_{M}<\min \left\{\check{I}_{1, M}, \check{I}_{2, M}\right\}\left(I_{M}>\max \left\{\check{I}_{1, M}, \check{I}_{2, M}\right\}\right)$. For all $\min \left\{\check{I}_{1, M}, \check{I}_{2, M}\right\} \leq I_{M} \leq$ $\max \left\{\check{I}_{1, M}, \check{I}_{2, M}\right\}$, the relationship between the two forces becomes ambiguous.

Moreover, comparing Eqs. (5.A.93) and (5.A.98) it is readily seen that the condition $\min \left\{\tilde{\alpha}_{L}, \tilde{\alpha}_{H}\right\}$ defines the binding thresholds for elasticity of substitution between tasks.

\section{A.7 Proof of Proposition 5.6}

Recall Eqs. (5.57) and (5.58) and rearrange slightly to obtain

$$
\begin{aligned}
\frac{\mathrm{d} \ln \omega_{L M}^{D}}{\mathrm{~d} \kappa} & =-\left(\frac{1}{\epsilon}-\frac{\epsilon-\sigma}{(\epsilon-1) \sigma}\left(1+\Gamma_{M}\right)\right) \Phi_{\kappa}+\frac{1}{\epsilon} \frac{1}{1-\kappa} \\
\frac{\mathrm{d} \ln \omega_{M H}^{D}}{\mathrm{~d} \kappa} & =\left(\frac{1}{\epsilon}-\frac{\epsilon-\sigma}{(\epsilon-1) \sigma}\left(1-\Delta_{M}\right)\right) \Phi_{\kappa}-\frac{1}{\epsilon} \frac{1}{1-\kappa}
\end{aligned}
$$


Now from the first terms on the right hand side, one obtains a threshold defined by

$$
\chi \equiv \frac{\epsilon^{2}+\sigma-2 \sigma \epsilon}{\epsilon(\epsilon-\sigma)},
$$

which sets a lower boundary for changes in the relative range of final goods. Thus, it follows that whenever

$$
\min \left\{\left|\Gamma_{M}\right|, \Delta_{M}\right\}>\chi
$$

the efficiency effect will be dominating. As defined by condition (5.A.98) in Appendix 5.A.6 it follows that

$$
\min \left\{\left|\Gamma_{M}\right|, \Delta_{M}\right\}=\min \left\{\tilde{\varepsilon}_{H}\left(1-z_{H}\right), \tilde{\varepsilon}_{L} z_{L}\right\}
$$

Moreover, since $\lim _{\kappa \rightarrow 0} \Phi_{\kappa}=\infty$, the efficiency effect will also dominate the labor supply effect (the second term on the right hand side) at low initial values of offshoring rate.

\section{B Derivation of equilibrium solutions}

\section{B.1 Derivation of equilibrium mass of monopolists $A_{k}$}

The derivation of the mass of monopolists in every technology mode can be derived as follows. First, notice that the perfectly competitive nature of the final goods market requires zero profits, i.e.

$$
p_{k}(z) y_{k}(z)=\zeta_{k} X_{k}, \quad \forall \quad k=\{L, M, H\}
$$

Using Eqs. (5.5) and (5.6), it follows that the price index of technology mode $k$ has to be equal to its marginal cost

$$
P_{k}=\zeta_{k},
$$

a result that has to hold in any perfectly competitive market. Now, utilizing the equilibrium value of $\zeta_{k}$ from Eq. (5.15), the equilibrium price index can be expressed as

$$
P_{k}=\zeta_{k}=\frac{\epsilon}{\epsilon-1} A_{k}^{\frac{1}{1-\epsilon}} \tilde{w}_{k} .
$$

From the total budget constraint for any final good producer $z$ using technology mode $k$ requires

$$
p_{k}(z) y_{k}(z)=w_{k}^{F} n_{k}^{F}+w_{k}^{D} n_{k}^{D}=\frac{1}{\mathcal{S}_{k}}\left(w_{k}^{F} N_{k}^{F}+w_{k}^{D} N_{k}^{D}\right),
$$

where the second equality follows from Eq. (5.23).

Utilize Eqs. (5.5) and (5.6) in the left hand side of the previous equation and manipulate to obtain

$$
P_{k} A_{k}^{\frac{\epsilon}{\epsilon-1}} x_{k}=\frac{w_{k}^{D} N_{k}^{D}}{\mathcal{S}_{k}}\left(1+\frac{w_{k}^{F}}{w_{k}^{D}} \frac{N_{k}^{F}}{N_{k}^{D}}\right) .
$$

Next, use the FE condition (5.14), and Eq. (5.B.101) to substitute for $x_{M}$ and $P_{M}$ in the 
previous equation, respectively, so that after some manipulation we obtain

$$
A_{k} \epsilon f_{k} \tilde{w}_{k}=\frac{w_{k}^{D} N_{k}^{D}}{\mathcal{S}_{k}}\left(1+\frac{w_{k}^{F}}{w_{k}^{D}} \frac{N_{k}^{F}}{N_{k}^{D}}\right) .
$$

Next, substitute Eq. (5.A.71) for $w_{k}^{F} / w_{k}^{D}$ in the right hand side and after simple manipulation we obtain

$$
\begin{aligned}
& A_{k} \epsilon f_{k} \tilde{w}_{k}=\frac{w_{k}^{D} N_{k}^{D}}{\mathcal{S}_{k}}\left(1+\left(\frac{\vartheta_{k}\left(I_{k}\right)}{\left(1-I_{k}\right)}\right)^{1 / \alpha}\left(\frac{N_{k}^{F}}{N_{k}^{D}}\right)^{-\frac{1}{\alpha}} \frac{N_{k}^{F}}{N_{k}^{D}}\right) \\
& A_{k} \epsilon f_{k} \tilde{w}_{k}=\frac{1}{\mathcal{S}_{k}} \frac{w_{k}^{D}\left(N_{k}^{D}\right)^{\frac{1}{\alpha}}}{\left(1-I_{k}\right)^{1 / \alpha}}\left(\left(1-I_{k}\right)^{1 / \alpha}\left(N_{k}^{D}\right)^{\frac{\alpha-1}{\alpha}}+\vartheta_{k}\left(I_{k}\right)^{\frac{1}{\alpha}}\left(N_{k}^{F}\right)^{\frac{\alpha-1}{\alpha}}\right)(5
\end{aligned}
$$

Recall the first order condition (5.A.63) and utilize Eqs. (5.A.66), (5.A.68), and (5.A.69) to obtain

$$
\begin{aligned}
w_{k}^{D}\left(l_{k}^{D}\right)^{1 / \alpha} & =\tilde{w}_{k} x_{k}^{1 / \alpha} \\
w_{k}^{D}\left(\frac{L_{k}}{1-I_{k}}\right)^{1 / \alpha} & =\tilde{w}_{k} \tilde{L}_{k}^{1 / \alpha}
\end{aligned}
$$

where recall

$$
\begin{aligned}
\tilde{L}_{k} & =\left(\vartheta_{k}\left(I_{k}\right)\left(L_{k}^{F}\right)^{\frac{\alpha-1}{\alpha}}+\left(1-I_{k}\right)^{1 / \alpha}\left(L_{k}^{D}\right)^{\frac{\alpha-1}{\alpha}}\right)^{\frac{\alpha}{\alpha-1}} \\
\vartheta_{k}\left(I_{k}\right) & \equiv\left(\int_{0}^{I_{k}} \gamma_{k}(i)^{1-\alpha} \mathrm{d} i\right)^{1 / \alpha}
\end{aligned}
$$

Now, substituting the total resource constraint condition (5.18) for $L_{M}$ and $L_{O}$, we obtain

$$
w_{k}\left(\frac{N_{k}^{D}}{1-I_{k}}\right)^{1 / \alpha}=\tilde{w}_{k} \tilde{N}_{k}^{1 / \alpha} .
$$

Then, substituting (5.B.103) for $w_{k}^{D}\left(\frac{N_{k}^{D}}{1-I_{k}}\right)^{1 / \alpha}$ in (5.B.102) and rearranging slightly yields

$$
A_{k}=\frac{\tilde{N}_{k}}{\epsilon f_{k} \mathcal{S}_{k}}
$$

\section{B.2 Derivation of aggregate sectoral output}

The derivation of the aggregate sectoral output is illustrated for the low-tech goods. The aggregation over the range of all final goods using the other two types of technology can be derived similarly. Define the total revenue using low-quality technology as

$$
P_{L} Y_{L}=\int_{0}^{z_{L}} p(z) y(z) \mathrm{d} z .
$$


Utilizing Eqs. (5.5) and (5.6) yields

$$
\begin{aligned}
P_{L} Y_{L} & =\int_{0}^{z_{L}} p(z) \varphi_{L}(z) X_{L} \mathrm{~d} z \\
& =\int_{0}^{z_{L}} P_{L} X_{L} \mathrm{~d} z \\
& =P_{L} z_{L} X_{L}
\end{aligned}
$$

Then, by equilibrium condition (5.21), it follows

$$
Y_{L}=A_{L}^{\frac{1}{\epsilon-1}} \tilde{N}_{L}
$$

Following the same steps, we obtain

$$
\begin{aligned}
& Y_{M}=A_{M}^{\frac{1}{\epsilon-1}} \tilde{N}_{M}, \\
& Y_{H}=A_{H}^{\frac{1}{\epsilon-1}} \tilde{N}_{H}
\end{aligned}
$$

\section{B.3 Derivation of relative wages}

To derive the relative wages structure for native workers, we proceed as follows. Recall the first order condition defining the final goods demand, Eq. (5.2). Now, multiplying both sides by $p(z)$ and noticing that from Eqs. (5.6), (5.26), and (5.27) it follows $p_{L}(z) y_{L}(z)=$ $P_{L} Y_{L} / z_{L}$ and $p_{M}(z) y_{M}(z)=P_{M} Y_{M} /\left(z_{H}-z_{L}\right)$, and $p_{H}(z) y_{H}(z)=P_{H} Y_{H} /\left(1-z_{H}\right)$, we get

$$
\begin{aligned}
\frac{P_{L} Y_{L}}{z_{L}} & =Y P_{L}^{1-\sigma} \varphi_{L}(z)^{\sigma-1}, \quad \forall z \in\left\{0, z_{L}\right\} \\
\frac{P_{M} Y_{M}}{z_{H}-z_{L}} & =Y P_{M}^{1-\sigma} \varphi_{M}\left(z^{\prime}\right)^{\sigma-1}, \quad \forall z^{\prime} \in\left\{z_{L}, z_{H}\right\} \\
\frac{P_{H} Y_{H}}{1-z_{H}} & =Y P_{H}^{1-\sigma} \varphi_{H}\left(z^{\prime \prime}\right)^{\sigma-1}, \quad \forall z^{\prime \prime} \in\left\{z_{H}, 1\right\}
\end{aligned}
$$

Rearranging and taking the ration with respect to $k=M$, we get the relative aggregate demand for final goods

$$
\begin{aligned}
\frac{P_{L}}{P_{M}} & =\left(\frac{z_{L}}{z_{H}-z_{L}}\right)^{\frac{1}{\sigma}}\left(\frac{Y_{L}}{Y_{M}}\right)^{-\frac{1}{\sigma}} \Lambda_{L}\left(z, z^{\prime}\right), \\
\frac{P_{M}}{P_{H}} & =\left(\frac{z_{H}-z_{L}}{1-z_{H}}\right)^{\frac{1}{\sigma}}\left(\frac{Y_{M}}{Y_{H}}\right)^{-\frac{1}{\sigma}} \Lambda_{H}\left(z^{\prime}, z^{\prime \prime}\right),
\end{aligned}
$$


where $\Lambda_{L}\left(z, z^{\prime}\right) \equiv\left(\frac{\varphi_{L}\left(z^{\prime}\right)}{\varphi_{M}\left(z^{\prime \prime}\right)}\right)^{\sigma-1}$ and $\Lambda_{H}\left(z^{\prime}, z^{\prime \prime}\right) \equiv\left(\frac{\varphi_{M}\left(z^{\prime}\right)}{\varphi_{H}\left(z^{\prime \prime}\right)}\right)^{\sigma-1} \cdot 11 \quad$ Next, use the equilibrium outcomes (5.27) to substitute for $Y_{k}$ to obtain

$$
\begin{aligned}
& \frac{P_{L}}{P_{M}}=\left(\frac{z_{L}}{z_{H}-z_{L}}\right)^{\frac{1}{\sigma}}\left[\frac{\tilde{N}_{L}}{\tilde{N}_{M}}\left(\frac{A_{L}}{A_{M}}\right)^{\frac{1}{\epsilon-1}}\right]^{-\frac{1}{\sigma}} \Lambda_{L}\left(z, z^{\prime}\right), \\
& \frac{P_{M}}{P_{H}}=\left(\frac{z_{H}-z_{L}}{1-z_{H}}\right)^{\frac{1}{\sigma}}\left[\frac{\tilde{N}_{M}}{\tilde{N}_{H}}\left(\frac{A_{M}}{A_{H}}\right)^{\frac{1}{\epsilon-1}}\right]^{-\frac{1}{\sigma}} \Lambda_{H}\left(z^{\prime}, z^{\prime \prime}\right),
\end{aligned}
$$

Next, use the equilibrium condition (5.34) and take the ratio with respect to technology mode $k=M$ to obtain

$$
\begin{aligned}
\frac{\tilde{w}_{L}}{\tilde{w}_{M}} & =\frac{\zeta_{L}}{\zeta_{M}}\left(\frac{X_{L}}{X_{M}}\right)^{1 / \epsilon}\left(\frac{x_{L}}{x_{M}}\right)^{-1 / \epsilon} \\
\frac{\tilde{w}_{M}}{\tilde{w}_{H}} & =\frac{\zeta_{M}}{\zeta_{H}}\left(\frac{X_{M}}{X_{H}}\right)^{1 / \epsilon}\left(\frac{x_{M}}{x_{H}}\right)^{-1 / \epsilon}
\end{aligned}
$$

Now, recalling the symmetry assumption and using Eq. (5.16) to substitute for $\zeta_{k}$ we get

$$
\begin{aligned}
& \frac{\tilde{w}_{L}}{\tilde{w}_{M}}=\frac{P_{L}}{P_{M}}\left(\frac{A_{L}}{A_{M}}\right)^{1 /(\epsilon-1)} \\
& \frac{\tilde{w}_{M}}{\tilde{w}_{H}}=\frac{P_{M}}{P_{H}}\left(\frac{A_{M}}{A_{H}}\right)^{1 /(\epsilon-1)}
\end{aligned}
$$

Next, utilize Eqs. (5.B.105) and (5.B.106) in the previous equations to substitute for $P_{L} / P_{M}$ and $P_{M} / P_{H}$, respectively.

$$
\begin{aligned}
\frac{\tilde{w}_{L}}{\tilde{w}_{M}} & =\left(\frac{z_{L}}{z_{H}-z_{L}}\right)^{\frac{1}{\sigma}}\left[\frac{\tilde{N}_{L}}{\tilde{N}_{M}}\left(\frac{A_{L}}{A_{M}}\right)^{\frac{1}{\epsilon-1}}\right]^{-\frac{1}{\sigma}}\left(\frac{A_{L}}{A_{M}}\right)^{1 /(\epsilon-1)} \Lambda_{L}\left(z, z^{\prime}\right) \\
\frac{\tilde{w}_{M}}{\tilde{w}_{H}} & =\left(\frac{z_{H}-z_{L}}{1-z_{H}}\right)^{\frac{1}{\sigma}}\left[\frac{\tilde{N}_{M}}{\tilde{N}_{H}}\left(\frac{A_{M}}{A_{H}}\right)^{\frac{1}{\epsilon-1}}\right]^{-\frac{1}{\sigma}}\left(\frac{A_{M}}{A_{H}}\right)^{1 /(\epsilon-1)} \Lambda_{H}\left(z^{\prime}, z^{\prime \prime}\right)
\end{aligned}
$$

Now, using the equilibrium expression for $A_{k}$, Eq. (5.22) and manipulating slightly we obtain

$$
\begin{aligned}
\frac{\tilde{w}_{L}}{\tilde{w}_{M}} & =\left(\frac{z_{L}}{z_{H}-z_{L}}\right)^{\frac{1}{\sigma}}\left(\frac{z_{L}}{z_{H}-z_{L}}\right)^{-\frac{\sigma-1}{(\epsilon-1) \sigma}}\left(\frac{\tilde{N}_{L}}{\tilde{N}_{M}}\right)^{\frac{\sigma-\epsilon}{(\epsilon-1) \sigma}}\left(\frac{f_{L}}{f_{M}}\right)^{-\frac{\sigma-1}{(\epsilon-1) \sigma}} \Lambda_{L}\left(z, z^{\prime}\right) \\
\frac{\tilde{w}_{M}}{\tilde{w}_{H}} & =\left(\frac{z_{H}-z_{L}}{1-z_{H}}\right)^{\frac{1}{\sigma}}\left(\frac{z_{H}-z_{L}}{1-z_{H}}\right)^{\frac{-(\sigma-1)}{(\epsilon-1) \sigma}}\left(\frac{\tilde{N}_{M}}{\tilde{N}_{H}}\right)^{\frac{\sigma-\epsilon}{(\epsilon-1) \sigma}}\left(\frac{f_{M}}{f_{H}}\right)^{-\frac{\sigma-1}{(\epsilon-1) \sigma}} \Lambda_{H}\left(z^{\prime}, z^{\prime \prime}\right)
\end{aligned}
$$

Finally using the Eqs. (5.23) and (5.24) to substitute for $\tilde{N}_{k}$ and $\tilde{w}_{k}$, respectively, then we

\footnotetext{
${ }^{11}$ Notice that the relative final goods demand at the margins, $z_{L}$ and $z_{H}$, is undefined since producers are indifferent regarding the adoption of different production technology modes.
} 
can rewrite the previously derived equations in terms of relative wages of native workers, i.e.

$$
\begin{aligned}
& \frac{w_{L}^{D}}{w_{M}^{D}}=\left(\frac{z_{L}}{z_{H}-z_{L}}\right)^{\frac{\epsilon-\sigma}{(\epsilon-1) \sigma}}\left(\frac{\Theta_{L}\left(I_{L}\right)}{\Theta_{M}\left(I_{M}\right)}\right)^{\frac{\alpha(\epsilon-\sigma)}{(\epsilon-1) \sigma}-1}\left(\frac{1-I_{L}}{1-I_{M}}\right)^{\frac{(\epsilon-\sigma)}{(\epsilon-1) \sigma}}\left(\frac{N_{L}^{D}}{N_{M}^{D}}\right)^{\frac{(\sigma-\epsilon)}{(\epsilon-1) \sigma}}\left(\frac{f_{L}}{f_{M}}\right)^{-\frac{\sigma-1}{(\epsilon-1) \sigma}} \Lambda_{L}\left(z, z^{\prime}\right) \\
& \frac{w_{M}^{D}}{w_{H}^{D}}=\left(\frac{z_{H}-z_{L}}{1-z_{H}}\right)^{\frac{\epsilon-\sigma}{(\epsilon-1) \sigma}}\left(\frac{\Theta_{M}\left(I_{M}\right)}{\Theta_{H}\left(I_{H}\right)}\right)^{\frac{\alpha(\epsilon-\sigma)}{(\epsilon-1) \sigma}-1}\left(\frac{1-I_{M}}{1-I_{H}}\right)^{\frac{(\epsilon-\sigma)}{(\epsilon-1) \sigma}}\left(\frac{N_{M}^{D}}{N_{H}^{D}}\right)^{\frac{(\sigma-\epsilon)}{(\epsilon-1) \sigma}}\left(\frac{f_{M}}{f_{H}}\right)^{-\frac{\sigma-1}{(\epsilon-1) \sigma}} \Lambda_{H}\left(z^{\prime}, z^{\prime \prime}\right)
\end{aligned}
$$

\section{B.4 Derivation of immigration-induced changes in relative range of final goods}

\section{Low-skill immigration}

To obtain explicit solution for the terms $\tilde{\Gamma}_{L} \equiv \frac{\mathrm{d} \ln \left[z_{L} /\left(z_{H}-z_{L}\right)\right]}{\mathrm{d} \ln I_{L}}=\frac{\mathrm{d} \ln \mathcal{M}_{L}}{\mathrm{~d} \ln I_{L}} \frac{\mathrm{d} \ln \left[z_{L} /\left(z_{H}-z_{L}\right)\right]}{\mathrm{d} \ln \mathcal{M}_{L}}$ and $\tilde{\Delta}_{I_{L}} \equiv \frac{\mathrm{d} \ln \left[\left(z_{H}-z_{L}\right) /\left(1-z_{H}\right)\right]}{\mathrm{d} \ln I_{L}}=\frac{\mathrm{d} \ln \mathcal{M}_{L}}{\mathrm{~d} \ln I_{L}} \frac{\mathrm{d} \ln \left[\left(z_{H}-z_{L}\right) /\left(1-z_{H}\right)\right]}{\mathrm{d} \ln \mathcal{M}_{L}}$, we can utilize the results of the comparative statics in Eqs. (5.A.81) and (5.A.83) to obtain the following terms

$$
\begin{aligned}
\tilde{\Gamma}_{L} & =\frac{z_{H}}{z_{H}-z_{L}}\left(\frac{\mathrm{d} \ln z_{L}-\mathrm{d} \ln z_{H}}{\mathrm{~d} \ln I_{L}}\right) \\
& =\Psi_{L} \Gamma_{L}>0
\end{aligned}
$$

where $\Gamma_{L}=\frac{1}{\mathcal{D}_{J}}\left(\frac{\epsilon-1}{\epsilon} \frac{\tilde{\varepsilon}_{H} z_{H} z_{H}}{\left(z_{H}-z_{L}\right)}+\frac{z_{H}}{\left(1-z_{H}\right)\left(z_{H}-z_{L}\right)}\right)>0$, and

$$
\begin{aligned}
\tilde{\Delta}_{I_{L}} & =-\left(\frac{z_{L}}{z_{H}-z_{L}} \frac{\mathrm{d} \ln z_{L}}{\mathrm{~d} \ln I_{L}}-\frac{z_{H}\left(1-z_{L}\right)}{\left(1-z_{H}\right)\left(z_{H}-z_{L}\right)} \frac{\mathrm{d} \ln z_{H}}{\mathrm{~d} \ln I_{L}}\right) \\
& =\Psi_{L} \Delta_{L}<0,
\end{aligned}
$$

where $\Delta_{L}=-\frac{1}{\mathcal{D}_{J}}\left(\frac{\epsilon-1}{\epsilon} \frac{\tilde{\varepsilon}_{H} z_{H} z_{L}}{\left(z_{H}-z_{L}\right)}\right)<0$. It is immediately evident that the size of $\Gamma_{L}$ and $\Delta_{L}$ depends, on the one hand, on the degrees of $\tilde{\varepsilon}_{L}$ and $\tilde{\varepsilon}_{H}$. On the other hand, it depends on the initial level of task margin $I_{L}$, captured by $\Psi_{L}$. As shown in Appendix 4.A.3 of chapter 4 , it follows that $0<\Gamma_{L}<1$ and $0<\left|\Delta_{L}\right|<1$ for $0<\left\{\tilde{\varepsilon}_{L}, \tilde{\varepsilon}_{H}\right\}<\infty$.

Moreover, both the labor supply effect, $I_{L} /\left(1-I_{L}\right)$, and the productivity effect, $\frac{\mathrm{d} \ln \Theta_{L}\left(I_{L}\right)}{\mathrm{d} \ln I_{L}}$, depend also on the initial level of the task margin. Thus, taking the limits and utilizing Eq. (5.A.78), we get that

$$
\begin{aligned}
\lim _{I_{L} \rightarrow 0} \frac{I_{L}}{1-I_{L}} & =0, \text { and } \lim _{I_{L} \rightarrow 1} \frac{I_{L}}{1-I_{L}}=\infty \\
\lim _{I_{L} \rightarrow 0} \frac{\mathrm{d} \ln \Theta_{L}\left(I_{L}\right)}{\mathrm{d} \ln I_{L}} & =0, \text { and } \lim _{I_{L} \rightarrow 1} \frac{\mathrm{d} \ln \Theta_{L}\left(I_{L}\right)}{\mathrm{d} \ln I_{L}}=-\mu_{\gamma_{L}}
\end{aligned}
$$




\section{Medium-skill immigration}

Following the same steps, we can solve for an explicit solution of the terms $\tilde{\Gamma}_{I_{M}} \equiv$ $\frac{\mathrm{d} \ln \left[z_{L} /\left(z_{H}-z_{L}\right)\right]}{\mathrm{d} \ln I_{M}}$ and $\tilde{\Delta}_{I_{M}} \equiv \frac{\mathrm{d} \ln \left[\left(z_{H}-z_{L}\right) /\left(1-z_{H}\right)\right]}{\mathrm{d} \ln I_{M}}$, we can utilize the results of the comparative statics derived in the main text, Eqs. (5.32) and (5.33), to obtain the following terms

$$
\begin{aligned}
\tilde{\Gamma}_{M} & =\frac{z_{H}}{z_{H}-z_{L}}\left(\frac{\mathrm{d} \ln z_{L}-\mathrm{d} \ln z_{H}}{\mathrm{~d} \ln I_{M}}\right) \\
& =\Psi_{M} \Gamma_{M}<0
\end{aligned}
$$

where $\Gamma_{M}=-\frac{1}{\mathcal{D}_{J}}\left(\frac{z_{H}}{\left(z_{H}-z_{L}\right)\left(1-z_{H}\right)}+\frac{\epsilon-1}{\epsilon}\left(\tilde{\varepsilon}_{L} z_{L}+\tilde{\varepsilon}_{H} z_{H}\right) \frac{z_{H}}{\left(z_{H}-z_{L}\right)}\right)<0$, and

$$
\begin{aligned}
\tilde{\Delta}_{M} & =\left(\frac{z_{H}\left(1-z_{L}\right)}{\left(1-z_{H}\right)\left(z_{H}-z_{L}\right)} \frac{\mathrm{d} \ln z_{H}}{\mathrm{~d} \ln I_{M}}-\frac{z_{L}}{z_{H}-z_{L}} \frac{\mathrm{d} \ln z_{L}}{\mathrm{~d} \ln I_{M}}\right) \\
& =\Psi_{M} \Delta_{M}>0
\end{aligned}
$$

where $\Delta_{M}=\frac{1}{\mathcal{D}_{J}}\left(\frac{z_{H}}{\left(1-z_{H}\right)\left(z_{H}-z_{L}\right)}+\frac{\epsilon-1}{\epsilon}\left(\frac{z_{H}\left(1-z_{L}\right)}{\left(1-z_{H}\right)\left(z_{H}-z_{L}\right)} \tilde{\varepsilon}_{L} z_{L}+\frac{z_{L}}{z_{H}-z_{L}} \tilde{\varepsilon}_{H} z_{H}\right)\right)>0$ Now, utilizing the results in (5.A.92) for $k=H$ and given $0<\left\{\tilde{\varepsilon}_{L}, \tilde{\varepsilon}_{M}\right\}<\infty$, we get that

$$
\begin{gathered}
\lim _{I_{M} \rightarrow 0} \Psi_{M}=0 \Rightarrow \lim _{I_{M} \rightarrow 0} \tilde{\Delta}_{M}=-\tilde{\Gamma}_{M}=\infty \\
\lim _{I_{M} \rightarrow 1} \Psi_{M}=\infty \Rightarrow \lim _{I_{M} \rightarrow 1} \tilde{\Delta}_{M}=\tilde{\Gamma}_{M}=0
\end{gathered}
$$

Moreover, from Eqs. (5.B.109) and (5.B.110), we get that

$$
\begin{aligned}
\Delta_{M} & \lesseqgtr\left|\Gamma_{M}\right| \\
\frac{\tilde{\varepsilon}_{L}}{1-z_{H}} & \lesseqgtr \frac{\tilde{\varepsilon}_{H}}{z_{H}}
\end{aligned}
$$

implying that the magnitude of changes in the technology margin $z_{L}$ relative to $\left(1-z_{H}\right)$ depends on the size of the elasticities at the respective margins. That is, if $\frac{\tilde{\varepsilon}_{L}}{1-z_{H}}<\frac{\tilde{\varepsilon}_{H}}{z_{H}}$ the adoption between $L$ and $M$ technology modes in the neighborhood of $z_{L}$ is easier relative to that between $M$ and $H$ technology modes in the neighborhood of $z_{H}$.

\section{High-skill immigration}

Following the same steps, we can solve for an explicit solution of the terms $\tilde{\Gamma}_{H} \equiv$ $\frac{\mathrm{d} \ln \left[z_{L} /\left(z_{H}-z_{L}\right)\right]}{\mathrm{d} \ln I_{H}}$ and $\tilde{\Delta}_{I_{H}} \equiv \frac{\mathrm{d} \ln \left[\left(z_{H}-z_{L}\right) /\left(1-z_{H}\right)\right]}{\mathrm{d} \ln I_{H}}$, we can utilize the results of the comparative statics in Eqs. (5.A.85) and (5.A.87) to obtain the following terms

$$
\begin{aligned}
\tilde{\Gamma}_{H} & =\frac{z_{H}}{z_{H}-z_{L}}\left(\frac{\mathrm{d} \ln z_{L}-\mathrm{d} \ln z_{H}}{\mathrm{~d} \ln I_{H}}\right) \\
& =\Psi_{H} \Gamma_{H}>0
\end{aligned}
$$


where $\Gamma_{H}=\frac{1}{\mathcal{D}_{J}}\left(\frac{\epsilon-1}{\epsilon} \frac{\tilde{\varepsilon}_{L}}{\left(z_{H}-z_{L}\right) z_{L}}\right)>0$, and

$$
\begin{aligned}
\tilde{\Delta}_{I_{H}} & =\left(\frac{z_{H}\left(1-z_{L}\right)}{\left(1-z_{H}\right)\left(z_{H}-z_{L}\right)} \frac{\mathrm{d} \ln z_{H}}{\mathrm{~d} \ln I_{H}}-\frac{z_{L}}{z_{H}-z_{L}} \frac{\mathrm{d} \ln z_{L}}{\mathrm{~d} \ln I_{H}}\right) \\
& =\Psi_{H} \Delta_{H}<0
\end{aligned}
$$

where $\Delta_{H}=-\frac{1}{\mathcal{D}_{J}}\left(\frac{\epsilon-1}{\epsilon} \frac{\tilde{\varepsilon}_{L} z_{L}\left(1-z_{L}\right)}{\left(1-z_{H}\right)\left(z_{H}-z_{L}\right)}+\frac{z_{H}}{\left(z_{H}-z_{L}\right)\left(1-z_{H}\right)}\right)<0$.

Similarly, utilizing Eq. (5.A.78) it follows that both the labor supply effect, $I_{H} /\left(1-I_{H}\right)$, and the productivity effect, $\frac{\mathrm{d} \ln \Theta_{H}\left(I_{H}\right)}{\mathrm{d} \ln I_{H}}$ converge to the following limits

$$
\begin{aligned}
& \lim _{I_{H} \rightarrow 0} \frac{I_{H}}{1-I_{H}}=0, \text { and } \lim _{I_{H} \rightarrow 1} \frac{I_{H}}{1-I_{H}}=\infty \\
& \lim _{I_{H} \rightarrow 0} \frac{\mathrm{d} \ln \Theta_{H}\left(I_{H}\right)}{\mathrm{d} \ln I_{H}}=0, \quad \text { and } \quad \lim _{I_{H} \rightarrow 1} \frac{\mathrm{d} \ln \Theta_{H}\left(I_{H}\right)}{\mathrm{d} \ln I_{H}}=-\mu_{\gamma_{H}}
\end{aligned}
$$

\section{B.5 Impact of immigration on native wage structure}

\section{Low-skill immigration and native wage structure}

To compute the impact of low-skill immigration on the wage structure of native workers, recall the Eqs. (5.35) and (5.36), take logs and differentiate with respect to task margin $I_{L}$ to obtain

$$
\begin{aligned}
& \frac{\mathrm{d} \ln \left(w_{L}^{D} / w_{M}^{D}\right)}{\mathrm{d} \ln I_{L}}=\frac{\epsilon-\sigma}{(\epsilon-1) \sigma}\left(\frac{\mathrm{d} \ln \left(z_{L} /\left(z_{H}-z_{L}\right)\right)}{\mathrm{d} \ln I_{L}}\right)+\left(\frac{\alpha(\epsilon-\sigma)}{(\epsilon-1) \sigma}-1\right)\left(\frac{\mathrm{d} \ln \Theta_{L}\left(I_{L}\right)}{\mathrm{d} \ln I_{L}}\right)-\left(\frac{(\epsilon-\sigma)}{(\epsilon-1) \sigma}\right) \frac{I_{L}}{1-I_{L}} \\
& \frac{\mathrm{d} \ln \left(w_{M}^{D} / w_{H}^{D}\right)}{\mathrm{d} \ln I_{L}}=\frac{\epsilon-\sigma}{(\epsilon-1) \sigma}\left(\frac{\mathrm{d} \ln \left(\left(z_{H}-z_{L}\right) /\left(1-z_{H}\right)\right)}{\mathrm{d} \ln I_{L}}\right)
\end{aligned}
$$

Utilizing now Eqs. (5.B.107) and (5.B.108) and making use of the definition of the terms $\Psi_{L} \equiv-\frac{\mathrm{d} \ln \left[\Omega_{L}\left(I_{L}\right)^{\alpha}\left(1-I_{L}\right)\right]}{\mathrm{d} \ln I_{L}}$ and $\tilde{\Theta}_{L}\left(I_{L}\right) \equiv-\frac{\mathrm{d} \ln \Theta_{L}\left(I_{L}\right)}{\mathrm{d} \ln I_{L}}$ we get

$$
\begin{aligned}
\left(\frac{\mathrm{d} \ln \left(z_{L} /\left(z_{H}-z_{L}\right)\right)}{\mathrm{d} \ln I_{L}}\right) & =\Psi_{L} \Gamma_{L}=\left(\alpha \tilde{\Theta}_{L}\left(I_{L}\right)+\frac{I_{L}}{1-I_{L}}\right) \Gamma_{L}>0 \\
\left(\frac{\mathrm{d} \ln \left(\left(z_{H}-z_{L}\right) /\left(1-z_{H}\right)\right)}{\mathrm{d} \ln I_{L}}\right) & =\Psi_{L} \Delta_{L}=\left(\alpha \tilde{\Theta}_{L}\left(I_{L}\right)+\frac{I_{L}}{1-I_{L}}\right) \Delta_{L}<0
\end{aligned}
$$

Now utilizing these results in the previously derived equations and rearranging yield Eqs. (5.37) and (5.38) derived in the text.

\section{High-skill immigration and native wage structure}

Following the same steps we can compute the distributional impact of high-skill immigration for native workers. More precisely, take logs in Eqs. (5.35) and (5.36) 
and differentiate with respect to task margin $I_{H}$ to obtain

$$
\begin{aligned}
\frac{\mathrm{d} \ln \left(w_{L}^{D} / w_{M}^{D}\right)}{\mathrm{d} \ln I_{H}}= & \frac{\epsilon-\sigma}{(\epsilon-1) \sigma}\left(\frac{\mathrm{d} \ln \left(z_{L} /\left(z_{H}-z_{L}\right)\right)}{\mathrm{d} \ln I_{H}}\right) \\
\frac{\mathrm{d} \ln \left(w_{M}^{D} / w_{H}^{D}\right)}{\mathrm{d} \ln I_{L}}= & \frac{\epsilon-\sigma}{(\epsilon-1) \sigma}\left(\frac{\mathrm{d} \ln \left(\left(z_{H}-z_{L}\right) /\left(1-z_{H}\right)\right)}{\mathrm{d} \ln I_{H}}\right)-\left(\frac{\alpha(\epsilon-\sigma)}{(\epsilon-1) \sigma}-1\right)\left(\frac{\mathrm{d} \ln \Theta_{H}\left(I_{H}\right)}{\mathrm{d} \ln I_{H}}\right) \\
& +\left(\frac{(\epsilon-\sigma)}{(\epsilon-1) \sigma}\right) \frac{I_{H}}{1-I_{H}}
\end{aligned}
$$

Now utilize Eqs. (5.B.111) and (5.B.112) and making use of the definition of the terms $\Psi_{H} \equiv-\frac{\mathrm{d} \ln \left[\Omega_{H}\left(I_{H}\right)^{\alpha}\left(1-I_{H}\right)\right]}{\mathrm{d} \ln I_{H}}$ and $\tilde{\Theta}_{H}\left(I_{H}\right) \equiv-\frac{\mathrm{d} \ln \Theta_{H}\left(I_{H}\right)}{\mathrm{d} \ln I_{H}}$ to obtain

$$
\begin{aligned}
\left(\frac{\mathrm{d} \ln \left(z_{L} /\left(z_{H}-z_{L}\right)\right)}{\mathrm{d} \ln I_{H}}\right) & =\Psi_{H} \Gamma_{H}=\left(\alpha \tilde{\Theta}_{H}\left(I_{H}\right)+\frac{I_{H}}{1-I_{H}}\right) \Gamma_{H}>0 \\
\left(\frac{\mathrm{d} \ln \left(\left(z_{H}-z_{L}\right) /\left(1-z_{H}\right)\right)}{\mathrm{d} \ln I_{H}}\right) & =\Psi_{H} \Delta_{H}=\left(\alpha \tilde{\Theta}_{H}\left(I_{H}\right)+\frac{I_{H}}{1-I_{H}}\right) \Delta_{H}<0
\end{aligned}
$$

Substituting these results in the previously derived equations and rearranging yield Eqs. (5.41) and (5.42) derived in the text.

\section{Medium-skill immigration and native wage structure}

To obtain the distributional impact of medium-skill immigration for native workers, take logs in Eqs. (5.35) and (5.36) and differentiate with respect to task margin $I_{M}$ to obtain

$$
\begin{aligned}
\frac{\mathrm{d} \ln \left(w_{L}^{D} / w_{M}^{D}\right)}{\mathrm{d} \ln I_{M}}= & \frac{\epsilon-\sigma}{(\epsilon-1) \sigma}\left(\frac{\mathrm{d} \ln \left(z_{L} /\left(z_{H}-z_{L}\right)\right)}{\mathrm{d} \ln I_{M}}\right)-\left(\frac{\alpha(\epsilon-\sigma)}{(\epsilon-1) \sigma}-1\right)\left(\frac{\mathrm{d} \ln \Theta_{M}\left(I_{M}\right)}{\mathrm{d} \ln I_{M}}\right) \\
& +\left(\frac{(\epsilon-\sigma)}{(\epsilon-1) \sigma}\right) \frac{I_{M}}{1-I_{M}} \\
\frac{\mathrm{d} \ln \left(w_{M}^{D} / w_{H}^{D}\right)}{\mathrm{d} \ln I_{L}}= & \frac{\epsilon-\sigma}{(\epsilon-1) \sigma}\left(\frac{\mathrm{d} \ln \left(\left(z_{H}-z_{L}\right) /\left(1-z_{H}\right)\right)}{\mathrm{d} \ln I_{M}}\right)+\left(\frac{\alpha(\epsilon-\sigma)}{(\epsilon-1) \sigma}-1\right)\left(\frac{\mathrm{d} \ln \Theta_{M}\left(I_{M}\right)}{\mathrm{d} \ln I_{M}}\right) \\
& -\left(\frac{(\epsilon-\sigma)}{(\epsilon-1) \sigma}\right) \frac{I_{M}}{1-I_{M}}
\end{aligned}
$$

Now utilize Eqs. (5.B.109) and (5.B.110) and making use of the definition of the terms $\Psi_{M} \equiv-\frac{\mathrm{d} \ln \left[\Omega_{M}\left(I_{M}\right)^{\alpha}\left(1-I_{M}\right)\right]}{\mathrm{d} \ln I_{M}}$ and $\tilde{\Theta}_{M}\left(I_{M}\right) \equiv-\frac{\mathrm{d} \ln \Theta_{M}\left(I_{M}\right)}{\mathrm{d} \ln I_{M}}$ to obtain

$$
\begin{aligned}
\left(\frac{\mathrm{d} \ln \left(z_{L} /\left(z_{H}-z_{L}\right)\right)}{\mathrm{d} \ln I_{M}}\right) & =\Psi_{M} \Gamma_{M}=\left(\alpha \tilde{\Theta}_{M}\left(I_{M}\right)+\frac{I_{M}}{1-I_{M}}\right) \Gamma_{M}<0 \\
\left(\frac{\mathrm{d} \ln \left(\left(z_{H}-z_{L}\right) /\left(1-z_{H}\right)\right)}{\mathrm{d} \ln I_{M}}\right) & =\Psi_{M} \Delta_{M}=\left(\alpha \tilde{\Theta}_{M}\left(I_{M}\right)+\frac{I_{M}}{1-I_{M}}\right) \Delta_{M}>0
\end{aligned}
$$

Substituting these results in the previously derived equations and rearranging yield Eqs. (5.39) and (5.40) derived in the text. 


\section{B.6 Derivation of $\hat{A}_{M}$}

Recall the zero profit condition

$$
p_{M}(z) y_{M}(z)=\frac{\tilde{w}_{M} \tilde{N}_{M}+w_{O} N_{O}}{z_{H}-z_{L}}
$$

Utilizing Eq. (5.6) in the right hand side and manipulating the left hand side slightly yields

$$
P_{M} \hat{X}_{M}=\frac{\tilde{w}_{M} \tilde{N}_{M}}{z_{H}-z_{L}}\left[1+\frac{w_{O}}{\tilde{w}_{M}} \frac{N_{O}}{\tilde{N}_{M}}\right]
$$

Now perfect competition in the final goods market requires $P_{M}=\hat{\zeta}_{M}$ and by symmetry it follows that $\hat{X}_{M}=A_{M}^{\frac{\epsilon}{\epsilon-1}}\left(\kappa x_{O}^{\frac{\epsilon-1}{\epsilon}}+(1-\kappa) x_{M}^{\frac{\epsilon-1}{\epsilon}}\right)^{\frac{\epsilon}{\epsilon-1}}$. Since the extent of offshoring is exogenously given, the FE condition (5.14) applies to both type of firms producing intermediates at home and abroad. Substituting these observations together with Eq. (5.49) and the markup-pricing in the left hand side of the previously derived equation yields

$$
\epsilon A_{M} \hat{w}_{M} f_{M}=\frac{\tilde{w}_{M} \tilde{N}_{M}}{z_{H}-z_{L}}\left[1+\frac{w_{O}}{\tilde{w}_{M}} \frac{N_{O}}{\tilde{N}_{M}}\right]
$$

where $\hat{w}_{M}$ denotes the marginal cost index of $\hat{N}_{M}$ and is defined by

$$
\hat{w}_{M}=\left[\kappa w_{O}^{1-\epsilon}+(1-\kappa) \tilde{w}_{M}^{1-\epsilon}\right]^{\frac{1}{1-\epsilon}} .
$$

Next substitute Eq. (5.46) for $w_{O} / \tilde{w}_{M}$ in the left hand side and manipulate to obtain

$$
\hat{\zeta}_{M} A_{M}^{\frac{\epsilon}{\epsilon-1}}(\epsilon-1) f_{M}=\frac{\tilde{w}_{M} \tilde{N}_{M}^{1 / \epsilon}}{\left(z_{H}-z_{L}\right)(1-\kappa)^{1 / \epsilon}}\left[\kappa^{\frac{1}{\epsilon}} N_{O}^{\frac{\epsilon-1}{\epsilon}}+(-\kappa)^{\frac{1}{\epsilon}} \tilde{N}_{M}^{\frac{\epsilon-1}{\epsilon}}\right]
$$

Now from the optimization problem

$$
\min _{\tilde{N}_{M}, N_{O}} \tilde{w}_{M} \tilde{N}_{M}+w_{O} N_{O} \text { s.t. } \hat{N}_{M}=\left(\kappa^{\frac{1}{\epsilon}} N_{O}^{\frac{\epsilon-1}{\epsilon}}+(-\kappa)^{\frac{1}{\epsilon}} \tilde{N}_{M}^{\frac{\epsilon-1}{\epsilon}}\right)^{\frac{\epsilon}{\epsilon-1}}
$$

the first order conditions imply

$$
\begin{aligned}
& \tilde{w}_{M}=\hat{w}_{M}\left(\frac{\hat{N}_{M}}{\tilde{N}_{M}}\right)^{\frac{1}{\epsilon}}(1-\kappa)^{1 / \epsilon} \\
& w_{O}=\hat{w}_{M}\left(\frac{\hat{N}_{M}}{N_{O}}\right)^{\frac{1}{\epsilon}} \kappa^{1 / \epsilon},
\end{aligned}
$$

Utilizing the first-order condition (5.B.114) in the left hand side of (5.B.113), manipulating and solving with respect to $\hat{A}_{M}$ yields the solution derived in the text. 



\section{Bibliography}

Abraham, F., Konings, J., and Vanromelingen, S. (2009). The Effect of Globalization on Union Bargaining and Price-Cost Margins of firms. Review of World Economic, 145:13-36.

Acemoglu, D. (1998). Why do new technologies complement skills? directed technical change and wage inequality. The Quarterly Journal of Economics, 113(4):pp. 10551089 .

Acemoglu, D. (2002a). Directed technical change. The Review of Economic Studies, 69(4):781-809.

Acemoglu, D. (2002b). Technical change, inequality, and the labor market. Journal of Economic Literature, 40:7-72.

Acemoglu, D. and Autor, D. (2011). Chapter 12 - Skills, Tasks and Technologies: Implications for Employment and Earnings. In Ashenfelter, O. and Card, D., editors, Handbook of Labor Economics, volume 4, Part B, pages 1043-1171. Elsevier.

Acemoglu, D., Gancia, G., and Zilibotti, F. (2012). Offshoring and direct technical change. NBER, Working Paper 18595.

Acemoglu, D. and Zilibotti, F. (2001). Productivity differences. Quarterly Journal of Economics, 116:563-606.

Autor, D. H. and Dorn, D. (2009). This jobs are getting old: Measuring changes in job opportunities using occupation age structure. American Economic Review: Papers 86 Proceedings, 99(2):45-51.

Autor, D. H. and Dorn, D. (2013). The growth of low skill service jobs and the polarization of the U.S. labor market. American Economic Review, 103(5):1553-1597.

Autor, D. H., Dorn, D., Hanson, G. H., and Song, J. (2013). Trade adjustment: Worker level evidence. NBER working paper 19226.

Autor, D. H., Katz, L. F., and Kearney, M. S. (2006). The Polarization of the U.S. Labor Market. American Economic Review, 96(2):189-194.

Autor, D. H., Katz, L. F., and Kearney, M. S. (2008). Trend in U.S. wage inequality: Revising the revisionists. Review of Economics and Statistics, 90:300-323.

Autor, D. H., Katz, L. F., and Krueger, A. B. (1998). Computing Inequality: Have Computers Changed the Labor Market? The Quarterly Journal of Economics, 113(4):1169-1213.

Autor, D. H., Levy, F., and Murnane, R. J. (2003). The Skill Content of Recent 
Technological Change: An Empirical Investigation. The Quarterly Journal of Economics, 118:1279-1333.

Baldwin, R. and Robert-Nicoud, F. (2014). Trade-in-goods and trade-in-tasks: An integrating framework. Journal of International Economics, 92:51-62.

Baltagi, B. H. (2008). Econometric analysis of panel data.

Baumgarten, D., Geishecker, I., and Görg, H. (2013). Offshoring, tasks, and the skill-wage pattern. European Economic Review, 61.

Baumol, W. J. (1967). Macroeconomics of unbalanced growth: The anatomy of urban crisis. The American Economic Review, 57(3):415-426.

Beaudry, P., Doms, M., and Lewis, E. (2010). Should the personal computer be considered a technological revolution? evidence from us metropolitan areas. Journal of Political Economy, 118(5):988-1036.

Beaudry, P. and Green, D. A. (2003). Wages and employment in the United States and Germany: What explains the differences. The American Economic Review, 93(3):573602.

Becker, S. O., Ekholm, K., and Muendler, M.-A. (2013). Offshoring and the onshore composition of tasks and skills. Journal of International Economics, 90:91-106.

Beladi, H. (1990). Unemployment, Trickle Down, Effects and Regional Income Disparities. Regional Science and Urban Economics, 20:351-357.

Bhagwati, J., Panagariya, A., and Srinivasan, T. (2004). The muddles over outsourcing. Journal of Economic Perspectives, 18:93-114.

Bhagwati, J. N. and Rodriguez, C. (1975). Welfare-theoretical analysis of brain drain. Journal of Development Economics, 2:195-221.

Blanchflower, D. G., Saleheen, J., and Shadforth, C. (2007). The Impact of the Recent Migration from Eastern Europe on the UK Economy. IZA, DP No. 2615.

Blinder, A. S. (2006). Offshoring: The Next Industrial Revolution? Foreign Affairs, $85(($ March/April $))$.

Blinder, A. S. (2009a). How Many U.S. Jobs Might Be Offshorable? World Economics, $10(2): 41-78$.

Blinder, A. S. (2009b). Offshoring: Big deal, or business as usual? In B.M., F., editor, Offshring of Amercian Jobs. What Response from U.S. Economic Policy?, pages 1043 - 1171. MIT Press, Cambridge, Mass.

Blinder, A. S. (2009c). Offshoring: Big deal, or business as usual? In Friedman, B. M., editor, Offshring of Amercian Jobs. What Response from U.S. Economic Policy?, pages 19-60. MIT Press, Cambridge, Mass.

Boeri, T. and Brücker, H. (2005). Migration, Co-Ordination Failures and EU Enlargement. IZA DP No. 1600.

Bonfanti, S. and Xenogiani, T. (2014). Matching Economic Migration with Labour Market Needs, chapter Migrants' skills: Use, mismatch and labour market outcomes - A first exploration of the International Survey of Adult Skills (PIAAC). OECD Publishing, Paris.

Booth, A. L. (1995). The Economics of the Trade Union. Cambridge University Press.

Borghans, L. and de Grip, A., editors (2000). The Overeducated Worker? The Economics of Skill Utilization. Edward Elgar, Cheltenham, UK.

Borghans, L., ter Weel, B., and Weinberg, B. A. (2008). Interpersonal styles and labor market outcomes. The Journal of Human Resources, XLIII(4).

Borghans, L., ter Weel, B., and Weinberg, B. A. (2013). People skills and the labor- 
market outcomes of underrepresented groups. Industrial and Labor Relations Review, $\operatorname{xxx}(\mathrm{x})$.

Borjas, G. J. (1995). The economic benefits from immigration. Journal of Economic Perspectives, $9(2)$.

Borjas, G. J. (2003). The Labor Demand Curve is Downward Sloping: Reexamining the Impact of Immigration on the Labor Market. Quarterly Journal of Economics, 118:1335-1374.

Borjas, G. J., Freeman, R. B., and Katz, L. (1996). Searching for the effect of immigration on the labor market. American Economic Review, 86.

Borjas, G. J., Grogger, J., and Hanson, G. H. (2011). Substitution between Immigrants, Natives, and Skill Groups. NBER, Working Paper 17461.

Brücker, H., Capuano, S., and Marfouk, A. (2013). Education, gender and international migration: Insights from a Panel dataset 1980-2010. mimeo.

Brücker, H., Hauptman, A., Jahn, E., and Upward, R. (2014). Migration and imperfect labor markets: Theory and cross-country evidence from Denmark, Germany and the UK. European Economic Review, 66:205-225.

Brücker, H. and Jahn, E. J. (2011). Migration and Wage-Setting: Reassessing the Labor Market Effects of Migration. Scandinavian Journal of Economics.

Brücker, H., Hauptmann, A., Jahn, E. J., and Upward, R. (2014). Migration and imperfect labor markets: Theory and cross-country evidence from Denmark, Germany and the UK. European Economic Review, 66:205-225.

Brynin, M. and Longhi, S. (2009). Overqualification: Major or minor mismatch? Economics of Education Review, 28:114-121.

Cahuc, P. and Zylberberg, A. (2004). Labor Economics. MIT Press, Cambridge, MA.

Card, D. (1990). The impact of the mariel boatlift on the miami labor market. Industrial and Labor Relations Review, 43(2):245-257.

Card, D. (2009). Immigration and inequality. American Economic Review, Papers and Proceedings, 99:1-21.

Caselli, F. (1999). Technological revolutions. American economic review, 89(1):78-102.

Chiswick, B. R. (1978). The effect of americanization on the earnings of foreign-born men. The Journal of Political Economy, pages 897-921.

Chiswick, B. R. and Miller, P. W. (2009). The international transferability of immigrants' human capital. Economics of Education Review, 28(2):162-169.

Chiswick, B. R. and Miller, P. W. (2012). Negative and positive assimilation, skill transferability, and linguistic distance. Journal of Human Capital, 6(1):35-55.

Cortes, P. (2008). The Effect of Low-Skilled Immigration on U.S. Prices: Evidence from CPI Data. Journal of Political Economy, 116(3):381-422.

Costinot, A. and Vogel, J. (2010). Matching and Inequality in the World Economy. Journal of Political Economy, 118(4):747-786.

Damas de Matos, A. and Liebig, T. (2014). Matching Economic Migration with Labour Market Needs, chapter The qualifications of immigrants and their value in the labour market. OECD Publishing, Paris.

Docquier, F. (2006). Brain drain and inequality across nations. IZA DP No. 2440.

Docquier, F. and Rapoport, H. (2012). Globalization, brain drain, and development. Journal of Economic Literature, 50:681-730.

Dornbusch, R., Fischer, S., and Samuelson, P. A. (1977). Comparative advantage, trade, and payments in a Ricardian model with a continuum of goods. The American 
Economic Review, 67(5):823-839.

Drinkwater, S., Eade, J., and Garapich, M. (2009). Poles Apart? EU Enlargement and the Labour Market Outcomes of Immigrants in the United Kingdom. International Migration, 47:161-190.

D'Amuri, F., Ottaviano, G. I. P., and Peri, G. (2010). The Labor Market Impact of Immigration in Wstern Germany in the 1990s. European Economic Review, 54:550 570.

Dupuy, A. (2012). A microfoundation for production functions: Assignment of heterogeneous workers to heterogeneous jobs. Economica, 79:534-556.

Dustmann, C., Fabbri, F., and Preston, I. (2005). The Impact of Immigration on the British Labour Market. The Economic Journal, 115:324-341.

Dustmann, C., Frattini, T., and Preston, I. (2013). The Effect of Immigration along the Distribution of Wages. Review of Economic Studies, 80(1):145-173.

Dustmann, C. and Glitz, A. (2012). How do industries and firms respond to changes in local labor supply? IZA Discussion Paper No.6257.

Dustmann, C., Glitz, A., and Frattini, T. (2008). The Labour Market Impact of Immigration. Oxford Review of Economic Policy, 24(3):477-494.

Dustmann, C., Ludsteck, J., and Schönberg, U. (2009). Revisiting the German Wage Structure. Quarterly Journal of Economics, 124(2):843-881.

Dustmann, C. and Preston, I. (2007). Racial and Economic Factors in Attitudes to Immigration. The B.E. Journal of Economic Analysis 8 Policy, 7:1-39.

Dustmann, C. and Schönberg, U. (2009). Training and Union Wages. Review of Economics and Statistics, 91(2):363-376.

Ebenstein, A., Harrison, A., McMillan, M., and Phillips, S. (2014). Estimating the Impact of Trade and Offshoring on American Workers Using the Current Population Surveys. The Review of Economics and Statistics, 96(4):581-595.

Egger, H., Kreickemeier, U., and Wrona, J. (2013). Offshoring domestic jobs. mimeo.

Ethier, W. (1982). National and International Returns to Scale in the Modern Theory of Iinternational Trade. The American Economic Review, 72(3):389-405.

Facchini, G. and Mayda, A. (2008). Attitudes and Migration Policy. Economic Policy, 23:652-713.

Feenstra, R. C. (2008). Offshoring in the Global Economy. The Ohlin Lecture, 2008.

Feenstra, R. C. and Hanson, G. H. (1996). Globalization, outsourcing, and wage inequality. The American Economic Review, 86(2):240-245.

Feenstra, R. C. and Hanson, G. H. (1999). The impact of outsourcing and high-technology capital on wages: Estimates for the united states, 1979-1990. The Quarterly Journal of Economics, 86(2):240-245.

Felbermayr, G. J., Geis, W., and Kohler, W. (2010). Restrictive Immigration Policy in Germany: Pains and Gains Foregone? Review of World Economics, 146:1-21.

Felbermayr, G. J. and Kohler, W. (2006). Immigration and Wages in General Equilibrium: A Theoretical Perspective. In Foders, F. and Langhammer, R., editors, Labor Mobility and the World Economy, pages 51-80. Springer.

Felbermayr, G. J. and Kohler, W. (2007). Immigration and Native Welfare. International Economic Review, 48:731-760.

Firpo, S., Fortin, N. M., and Lemieux, T. (2011). Occupational tasks and changes in the wage structure. IZA, Discussion Paper No. 5542.

Friedberg, R. M. (2000). You can't take it with you? immigrant assimilation and the 
portability of human capital. Journal of Labor Economics, 18(2):221-251.

Gandolfo, G. (2010). Economic Dynamics. Springer-Verlag, fourth edition. Study Edition. Garcia Pires, A. J. (2009). Brain drain and brain waste. mimeo.

Gauthier-Loiselle, M. and Hunt, J. (2008). How much does immigration boost innovation? NBER Working Paper No. 14312.

Ghosh, A., Mayda, A. M., and Ortega, F. (2014). The impact of skilled foreign workers on firms: An investigation of publicly traded us firms. IZA DP No. 8684.

Gibson, J. and McKenzie, D. (2011). Eight questions about brain drain. Journal of Economic Perspectives, 25:107-128.

Goldin, C. D. and Katz, L. F. (2009). The race between education and technology. Harvard University Press.

González, L. and Ortega, F. (2011). How do very open economies adjust to large immigration flows? Evidence from Spanish regions. Labour Economics, 18:57-70.

Goos, M. and Manning, A. (2007). Lousy and Lovely Jobs: The Rising Polarization of Work in Britain. Review of Economic Studies, 89:118-133.

Goos, M., Manning, A., and Salomons, A. (2009). Job polarization in Europe. American Economic Review: Papers \& Proceedings, 99:58-63.

Goos, M., Manning, A., and Salomons, A. (2011). Explaining job polarization: The roles of technology, offshoring and institutions. CES DP No. 11.34.

Goos, M., Manning, A., and Salomons, A. (2014). Explaining job polarization: Routinebiased technological change and offshoring. American Economic Review, 104:2509 2526 .

Grossman, G. M. and Rossi-Hansberg, E. (2008). Trading Task: A Simple Theory of Offshoring. American Economic Review, 98:1978-1997.

Gustafsson, P. and Segerstrom, P. (2010). Trade liberalization and productivity growth. Review of International Economics, 18:207-228.

Harrison, A. and McMillan, M. (2011). Offshoring Jobs? Multinationals and U.S. Manufacturing Employment. The Review of Economics and Statistics, 93(3):857-875.

Hartog, J. (2000). Over-education and earnings: Where are we, where should we go? Economics of Education Review, 19:131-147.

Hartwig, J. (2011). Testing the Baumol-Nordhaus Model with EU KLEMS Data. Review of Income and Wealth, 57:471-489.

Helpman, E., Itskhoki, O., Redding, S., and Muendler, M.-A. (2014). Trade and inequality: From theory to estimation. mimeo.

Hummels, D., Jørgensen, R., Munch, J., and Xiang, C. (2014). The Wage Effects of Offshoring: Evidence from Danish Matched Worker-Firm Data. American Economic Review, 104(6).

IMF (2007). World Economic Outlook, April 2007: Spillovers and Cycles in the Global Economy. SOPEMI-Report.

Iranzo, S. and Peri, G. (2009). Migration and trade: Theory with an application to the Eastern-Western European integration. Journal of International Economics, 79:1-19.

Jensen, J. B. and Kletzer, L. G. (2010). Measuring Tradable Services and the Task Content of Offshorable Services Jobs, pages 309-335. University of Chicago Press.

Jones, R. W. (1965). The Structure of Simple General Equilibrium Models. The Journal of Political Economy, 73:557-572.

Jones, R. W. and Kierzkowski, H. (1990). The role of services in production and international trade: A theoretical framework. In Jones, R. W. and Krueger, A. O., 
editors, The Political Economy of International Trade, pages 31-48. Basil Blackwell, Oxford.

Jones, R. W. and Kierzkowski, H. (2001). A framework for fragmentation. In Arndt, S. and Kierzkowski, H., editors, Fragmentation: New Production Patterns in the World Economy, pages 17-45. Oxford University Press, New York.

Kahanec, M. and Zimmermann, K. F. (2010). Migration in an Enlarged EU: A Challenging Solution? In Keereman, F. and Szekely, I., editors, Five Years of an Enlarged EU: A Positive Sum Game, pages 63-94. Springer.

Katz, L. F. and Murphy, K. M. (1992). Changes in Relative Wages, 1963-1987: Supply and Demand Factors. The Quarterly Journal of Economics, 107:35-78.

Kohler, W. (2004a). Aspects of international fragmentation. Review of International Economics, 12:793-816.

Kohler, W. (2004b). International outsourcing and factor prices with multistage production. Economic Journal, 114:166-185.

Kohler, W. and Wrona, J. (2011). Offshoring Tasks, yet Creating Jobs? University of Tübingen, Working Paper No. 12.

Krugman, P. R. (1979). Increasing returns, monopolistic competition, and international trade. Journal of International Economics, 9:469-479.

Layard, R., Nickell, S., and Jackman, R. (2005). Unemployment: Macroeconomic Performance and the Labour Market. Oxford University Press.

Lemieux, T. (2008). The changing nature of wage inequality. Journal of Population Economics, 21(1):21-48.

Lewis, E. (2011). Immigration, skill mix, and capital skill complementarity. The Quarterly Journal of Economics, 126(2):1029-1069.

Lewis, E. G. (2003). Local open economies within the U.S.: How do industries respond to immigration. Federal Reserve Bank of Philadelphia Working Paper No. 04-03.

Lewis, E. G. (2013). Immigration and production technology. Annual Review of Economics, 5:165-191.

Manacorda, M., Manning, A., and Wadsworth, J. (2011). The Impact of Immigration on the Structure of Male Wages: Theory and Evidence from Britain. Journal of the European Economic Association, 10:120-151.

Mankiw, N. G. and Swagel, P. (2006). The politics and economics of offshore outsourcing. Journal of Monetary Economics, 53(5):1027-1056.

Mattoo, Aaditya, I. C. N. a. z. (2008). Brain waste? educated immigrants in the us labor market. Journal of Development Economics, 87:255-269.

Mayda, A. (2006). Who is against Immgration? a Cross-Country investigation of Individual Attitudes toward Immigration. The Review of Economics and Statistics, $88(3): 510-530$.

Melitz, M. (2003). The Impact of Trade on Intra-Idustry Reallocations and Aggregate Industry Productivity. Econometrica, 71:1695-1725.

Michaels, G., Natraj, A., and Van Reenen, J. (2014). Has ICT Polarized Skill Demand? Evidence from Eleven Countries over Twenty-Five Years. The Review of Economics and Statistics, 96(1):60-77.

Moncarz, R. J., Wolf, M. G., and Wright, B. (2008). Service-providing occupations, offshoring, and the labor market. Monthly Labor Review, pages 71-86.

Muysken, J., Vallizadeh, E., and Ziesemer, T. (2015). Migration, Unemployment, and Skill Downgrading. The Scandinavian Journal of Economics, 117:403-451. 
Muysken, J. and Ziesemer, T. H. (2011). The Effect of Net Immigration on Economic Growth in an Ageing Economy: Transitory and Permanent Shocks. UNU-MERIT Working Papers, 2011-055. Maastricht University.

Neary, P. (1985). International factor mobility, minimum wage rates, and factor-price equalization: A synthesis. The Quarterly Journal of Economics, 100(3):551-570.

OECD (2007a). International Migration Outlook. OECD Publishing.

OECD (2007b). International Migration Outlook. OECD Publishing.

OECD (2013). OECD Employment Outlook 2013. OECD Publishing.

Olney, W. W. (2012). Offshoring, immigration, and the native wage distribution. Canadian Journal of Economics, 45:830-856.

Ottaviano, G. I. and Peri, G. (2008). Immigration and National Wages: Clarifying the Theory and the Empirics. NBER Working Paper No. 14188.

Ottaviano, G. I. and Peri, G. (2012). Rethinking the Effects of Immigration on Wages. Journal of the European Economic Association, (10):152-197.

Ottaviano, G. I. P., Peri, G., and Wright, G. C. (2013). Immigration, Offshoring and American Jobs. American Economic Review, 103(5):1925-59.

Peri, G. (2011). Rethinking the area approach: Immigrants and the labor market in California. Journal of International Economics, 84(1):1-14.

Peri, G. (2012). The effect of immigration on productivity: Evidence from us states. Review of Economics and Statistics, 94(1):348-358.

Peri, G. and Sparber, C. (2009). Task specialization, immigration, and wages. American Economic Journal: Applied Economics, 1(3):135-169.

Pierrard, O. and Sneessens, H. R. (2003). Low-Skilled Unemployment, Biased Technological Shocks and Job Competition. IZA DP No. 784.

Pissarides, C. A. (2000). Equilibrium Unemployment Theory. MIT Press, Cambrdige, MA, 2nd edition.

Ratha, D., Mohapatra, S., Özden, C., Plaza, S., Shaw, W., and Shimeles, A. (2011). Leveraging migration for Africa: remittances, skills, and investments. World Bank Publications.

Rodríguez-Clare, A. (2010). Offshoring in a Ricardian World. American Economic Journal: Macroeconomics, 2(2):227-258.

Romer, P. M. (1987). Growth based on increasing returns due to specialization. The American Economic Review, 77(2):56-62.

Scheve, K. F. and Slaughter, M. J. (2001). Labor market competition and individual preferences over immigration policy. The Review of Economics and Statistics, 83(1):133-145.

Shapiro, C. and Stiglitz, J. (1984). Equilibrium unemployment as a worker discipline device. American Economic Review, 75:433-444.

Siebert, H. (1997). Labor Market Rigidities: At the Root of Unemployment in Europe. Journal of Economic Perspectives, 11:37-54.

Sly, N. (2012). International productivity differences, trade and the distribution of factor endowments. Review of International Economics, 20:740-757.

Snower, D. J., Brown, A. J. G., and Merkl, C. (2009). Globalization and the welfare state: A review of Hans-Werner Sinn's Can Germany Be Saved? Journal of Economic Literature, 47:136-158.

Spence, M. (2011). Globalization and unemployment: The downside of integrating markets. 
Spitz-Oener, A. (2006). Technical change, job tasks, and rising educational demands: Looking outside the wage structure. Journal of Labour Economics, 24:235-270.

Stasz, C. (1997). Do employers need the skills they want? Evidence from technical work. Journal of Education and Work, 10(3):205-223.

Vallizadeh, E., Muysken, J., and Ziesemer, T. (2015). Offshoring of Medium-skill Jobs, Polarization, and Productivity Effect: Implications for Wages and Low-skill Unemployment. IAB-Discussion Papers, 7/2015.

Venn, D. (2009). Legislation, collective bargaining and enforcement: Updating the oecd employment protection indicators. http://www. oecd. org/els/workingpapers.

Weiss, M. and Garloff, A. (2009). Skill-Biased Technological Change and Endogenous Benefits: The Dynamics of Unemployment and Wage Inequality. Applied Economics, 43:811-821.

Wooldridge, J. M. (2002). Econometric analysis of cross section and panel data. MIT press.

Wright, G. C. (2014). Revisiting the employment impact of offshoring. European Economic Review, 66:63-83.

Yeaple, S. R. (2005). A simple model of firm heterogeneity, international trade, and wages. Journal of International Economics, 65:1-20.

Özden, C. (2006). Educated migrants: Is there brain waste? In Özden, . and Schiff, M., editors, International Migration, Remittances and the Brain Drain, pages 227-244. World Bank and Palgrave MacMillan.

Ziesemer, T. (2011). Developing Countries' Net-migration: The Impact of Economic Opportunities, Disasters, Conflicts, and Political Instability. International Economic Journal, 25:373-386.

Ziesemer, T. (2012). Worker remittances, migration, accumulation and growth in poor developing countries: Survey and analysis of direct and indirect effects. Economic Modelling, 29:103-118. 


\section{Samenvatting (Summary in Dutch)}

Gedurende de laatste twee decennia zijn nationale arbeids- en goederenmarkten in steeds sterkere mate geïntegreerd met de wereldmarkt. Twee factoren spelen bij deze nieuwe globalisering een belangrijke rol. Enerzijds zorgen de vooruitgang in de informatieen communicatietechnologie, het toelaten van vrije kapitaalstromen en het opheffen van handelsbelemmeringen ervoor dat productie- en arbeidsprocessen steeds minder afhankelijk worden van tijd en plaats. Anderzijds hebben (drastische) institutionele en economische hervormingen in opkomende markten - zoals die van de BRIC- en voormalig Oostbloklanden - ertoe geleid dat deze landen zich ontwikkeld hebben tot sterke economieën, met groeiende investeringen in menselijk kapitaal, geavanceerde technologie, en bedrijfsinfrastructuur.

De sterke globalisering van goederen- en arbeidsmarkten leidt ertoe dat de geavanceerde economieën steeds meer gebruik maken van de nieuwe arbeid, door gebruik te maken van arbeidsmigranten of door gedeelten van het productieproces uit te besteden. Deze ontwikkeling krijgt veel aandacht in de media en in het beleid, en wordt vaak gezien als een bedreiging voor zittende werknemers, die hun banen zien verdwijnen naar het buitenland, of die nu in eigen land moeten concurreren met arbeidsmigranten. Dit proefschrift draagt bij aan deze discussie. Middels theoretische modellen wordt onderzocht hoe de arbeidsmarkt en de economie de stroom arbeidsmigranten en offshoring absorberen en hoe deze zich verder ontwikkelen.

\section{Hoofdstuk 2}

Hoofdstuk 2 biedt inzicht in mogelijke verdringingseffecten die arbeidsmigratie met zich meebrengt voor zittende arbeiders die laaggeschoold werk verrichten, aangezien de immigratie van laaggeschoolden leidt tot een verlaging van het algeheel vaardigheidsniveau van de beroepsbevolking (skill-downgrading). Hiertoe wordt een model ontwikkeld waarin de economie bestaat uit een dienstensector en een industriesector. In deze sectoren werken, respectievelijk, alleen laaggeschoolde en middelbaar geschoolde arbeiders. Hooggeschoolden zijn inzetbaar in beide sectoren. 
De analyses geven aan wat de gevolgen van immigratie zijn voor het aandeel laaggeschoolden op de arbeidsmarkt, als ook voor de werkloosheid onder deze groep arbeiders. Daarnaast wordt ook gekeken naar het belang van de structuur van de arbeidsmarkt. Twee systemen worden vergeleken: de ene arbeidsmarkt is flexibel, in de zin dat de werkloosheidsuitkeringen endogeen zijn, en beïnvloed worden door het beroep op deze voorzieningen. In deze setting leidt een hoger beroep tot lagere uitkeringen. De andere arbeidsmarkt is rigide, waarin de werkloosheidsuitkeringen als exogeen en nietveranderlijk beschouwd worden.

Uit de analyses komen twee interessante conclusies naar voren. Ten eerste, onder een flexibele arbeidsmarkt, leidt de skill-downgrading door immigratie van arbeiders tot een afname van de werkloosheid onder laaggeschoolde arbeiders. Ten tweede zien we dat de recente trend van immigratie van hooggeschoolden leidt tot een 6 tot 10 procent hoger $\mathrm{BBP}$ vergeleken met een situatie waarin deze immigratie niet had plaatsgevonden.

\section{Hoofdstuk 3}

Hoofdstuk 3 bespreekt de gevolgen van uitbesteding (offshoring) voor de inkomensverdeling en werkgelegenheid in het thuisland. Het doel is tweeledig. Ten eerste wordt gekeken naar de gevolgen voor de inkomensverdeling in een situatie van uitbesteding, waarbij verschillende groepen arbeiders geraakt worden door de uitbesteding van bepaalde taken. Ten tweede vallen door uitbesteding de banen van veel middelbaar geschoolde arbeiders weg. Deze arbeiders vinden nieuw werk in laaggeschoolde banen. Het is daarom van belang te kijken naar de indirecte effecten van deze herindeling op de werkloosheid van laaggeschoolde arbeiders.

De resultaten zijn gebaseerd op een theoretisch model met een heterogene verdeling van taken en vaardigheden (laag-, middelbaar-, en hooggeschoold), endogene uitbesteding van taken en delen van het productieproces, en tot slot ook indirecte effecten als gevolg van mobiliteit tussen taken en vaardigheidsgroepen. Hierbij wordt ook rekening gehouden met twee bronnen van frictie op de arbeidsmarkt. Er wordt gestart met een situatie waarin een minimumloon geldt, om vervolgens te komen tot een meer generiek verloop van aanpassingen in de arbeidsmarkt door een endogeen aanbod van laaggeschoolde arbeid toe te staan.

Uit de analyses komen verschillende nieuwe inzichten naar voren. Ten eerste zijn de gevolgen van het uitbesteden van laaggeschoolde taken te vergelijken met die van "skillbiased technological change" (cf. Acemoglu, 2002b). Worden middelbaargeschoolde taken uitbesteed aan andere economieën, dan ontstaat er een polarisatie in de lonen, waarbij laag en hoog geschoolden in het thuisland er relatief op vooruit gaan in vergelijking met de middelbaar geschoolden (zichtbaar in recente ontwikkelingen). Worden hooggeschoolde taken uitbesteed, dan zijn de gevolgen vergelijkbaar met die van "unskilled-biased technological change" (cf. Goldin and Katz, 2009).

Een andere bevinding is dat hoewel de uitbesteding leidt tot verdringing, de uitbesteding ook leidt tot productiviteitswinsten, doordat er efficiënter en tegen lagere marginale kosten geproduceerd kan worden. Het negatieve effect voor arbeiders die door verdringing 
op een lager niveau werken - en tegen een lager loon - wordt teniet gedaan door de winst in efficiëntie en productiviteit.

Tot slot wordt aangetoond dat de mate waarin uitbesteding een effect heeft op de werkloosheid onder laaggeschoolden sterk afhankelijk is van de mate van wederzijdse substitutie van middelbaar- en laaggeschoold werk.

\section{Hoofdstuk 4}

Hoofdstuk 4 gaat in op de relatie tussen immigratie en de daarmee samenhangende veranderingen in de structuur en inzet van technologie. Het doel is om te laten zien hoe de inzet van technologie verandert als gevolg van de veranderende verdeling van vaardigheden in de beroepsbevolking. Vervolgens wordt in beeld gebracht hoe deze technologische aanpassingen weer van invloed zijn op de inkomensverdeling. Het onderliggende theoretische model van de economie kenmerkt zich door heterogeniteit in bedrijven als ook in vaardigheden (laag-, middelbaar-, en hooggeschoold) en een endogene keuze voor technologie.

De analyses laten twee interessante resultaten zien. Ten eerste ontstaan veranderingen in de inkomensverdeling door een samenspel van twee effecten. Aan de ene kant ontstaat door immigratie een grotere potentiële beroepsbevolking. Een groeiend aandeel van een bepaald type arbeider drukt de lonen voor deze groep arbeiders door grotere onderlinge concurrentie. Anderzijds zorgt een instroom van arbeiders ervoor dat bedrijven zich specialiseren in technologie die complementair is met die groep arbeiders, hetgeen doorgaans een positief effect heeft op de lonen. Ten tweede laten de resultaten zien dat het relatieve belang van deze - in tegengestelde richting werkende - krachten afhangt van de mate van adoptie van nieuwe technologieën. De richting van beide effecten afzonderlijk wordt bepaald door de interactie tussen voorkeuren van consumenten en het niveau van marktmacht van de monopolistische concurrenten.

\section{Hoofdstuk 5}

Naast veranderingen in technologie, kunnen bedrijven ook hun organisatiestructuur aanpassen als gevolg van immigratie. Hoofdstuk 5 laat zien wat met de inkomens van de zittende arbeiders gebeurt als deze organisatorische aanpassingen in beschouwing worden genomen. Hiertoe wordt het economisch model van Hoofdstuk 4 op twee manieren uitgebreid. Ten eerste vindt een herverdeling van taken plaats binnen bedrijven, waarbij de aanname is dat zittende arbeiders relatief vaker worden ingezet op plekken waar communicatieve vaardigheden van belang zijn en immigranten vaker worden ingezet op handmatige klussen (cf. Peri and Sparber, 2009). Daarnaast wordt uitbesteding geïntroduceerd, waarbij wordt aangenomen dat met name de taken van middelbaar geschoolde arbeiders worden uitbesteed aan het buitenland (cf. Acemoglu and Autor, 2011).

De analyses leiden tot verschillende nieuwe inzichten. Ten eerste hebben immigratie en uitbesteding een effect op zowel de efficiëntie als het aanbod van arbeid, twee 
zaken die op elkaar inwerken. Ten tweede is te zien dat de relatieve grootte van het efficiëntie-effect door immigratie (door herverdeling van taken) en door uitbesteding (door herverdeling van banen) sterk afhangt van de startwaarden voor wat betreft de taken toebedeeld aan immigranten, als ook het aandeel van uitbesteding. Met lage beginwaarden van de scholingsgraad van taken domineert het efficiëntie-effect, met als gevolg hogere lonen voor de zittende arbeiders binnen eenzelfde vaardigheidsniveau. De richting en relatieve verhoudingen tussen de effecten van een groeiende beroepsbevolking en die van technologische aanpassingen zijn vergelijkbaar met die in Hoofdstuk 4, maar in een situatie waarin het heel eenvoudig is te schakelen tussen verschillende technologieën - waarin de effecten van een groeiende beroepsbevolking en die van technologische aanpassingen in evenwicht zijn - blijven de winsten in termen van efficiëntie - als gevolg van een herverdeling van taken en een reductie in de marginale kosten van productie - behouden. Dit laatste gegeven wijkt af van recente studies over de gevolgen van immigratie en uitbesteding (cf. Acemoglu et al., 2012; Ottaviano et al., 2013).

De resultaten tonen aan dat er significante aanpassingen zijn op macro niveau, met aanpassingen in technologie, als ook op micro niveau, met een herverdeling van taken en banen. 


\section{Curriculum Vitae}

Ehsan Vallizadeh was born on September 29, 1978 in Tehran, Iran. He started studying economics at the University of Bonn in 2002, where he obtained his Master of Science in Economics in October 2007, with a major in applied economic theory.

He gained his first experience in the world of research as assistant researcher at the Department of Economic Theory III and the Department of Business Administration I Finance at University of Bonn, before coming to Maastricht University, the Netherlands, as a PhD-candidate at the Department of Economics of the School of Business and Economics, in September 2008.

The results of his work as a doctoral candidate are collected in this dissertation and part of his thesis is published in international refereed academic journals. His primary research interests lie in Economics of Migration, International Economics and Applied Economic Theory. The results of his research have been presented at various international conferences, including annual meetings of the European Association of Labor Economists (2012), the European Society of Population Economics (2014), the Association of Southern European Economic Theorists (2015), the European Trade Study Group (2015), and the Italian Association of Labor Economists (2015).

As of January 2013, Ehsan holds a research position at the Department of International Comparisons and European Integration at the Institute for Employment Research (IAB) in Nuremberg, Germany. 
W

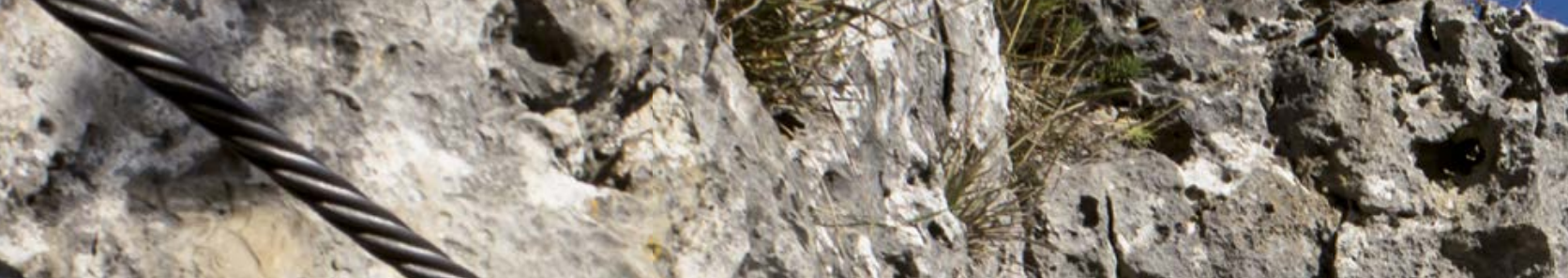
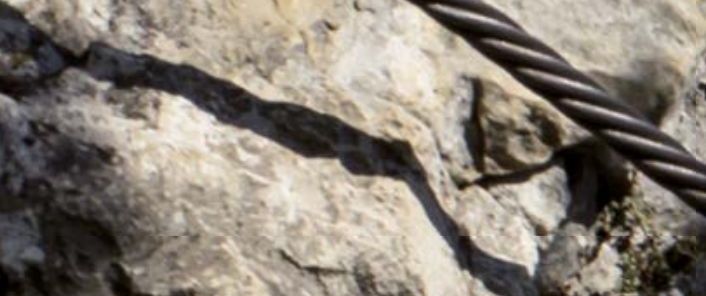

$\left.13 x^{2} x^{4}\right\}$

(r) 44

ati $-1,2$

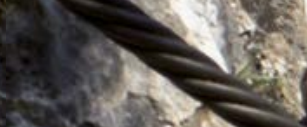

(2)

tace

L

4

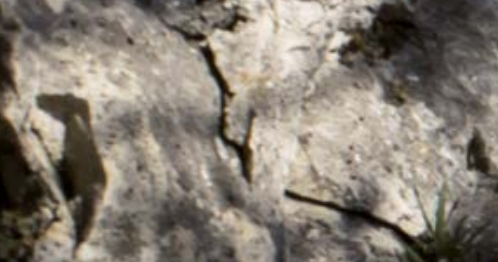

(x)

,

ant

a.s

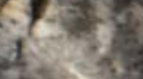

a

T. 13

x. 3 .

$x+30$

its. $x^{2}+\mathrm{l}^{5}+\overline{6}$

(2) 12 $\left.\operatorname{lom}^{4}\right)^{4}$

क.

(4) 8

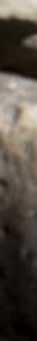
-5x 54 . 21 thice $x^{2}=$ $6 x^{2}+2$ isitet $y^{2} \times 1$ cherta 3.

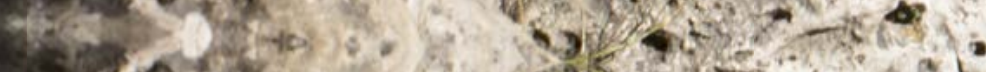

